Claudia Vogel

Markus Wettstein

Clemens Tesch-Römer Hrsg.

Frauen und Männer

in der zweiten

Lebenshälfte

Älterwerden im sozialen Wandel 
Frauen und Männer in der zweiten Lebenshälfte 
Claudia Vogel - Markus Wettstein -

Clemens Tesch-Römer (Hrsg.)

Frauen und Männer

in der zweiten

Lebenshälfte

Älterwerden im sozialen Wandel

Mit einem Grußwort der Bundesministerin Dr. Franziska Giffey 
Hrsg.

Claudia Vogel

Deutsches Zentrum für Altersfragen

Berlin, Deutschland

Markus Wettstein

Deutsches Zentrum für Altersfragen

Berlin, Deutschland

Clemens Tesch-Römer

Deutsches Zentrum für Altersfragen

Berlin, Deutschland

Der Deutsche Alterssurvey (DEAS) wird aus Mitteln des Bundesministeriums für Familie, Senioren, Frauen und Jugend (BMFSFJ) gefördert.

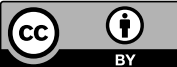

ISBN 978-3-658-25078-2 ISBN 978-3-658-25079-9 (eBook)
https://doi.org/10.1007/978-3-658-25079-9

Die Deutsche Nationalbibliothek verzeichnet diese Publikation in der Deutschen Nationalbibliografie; detaillierte bibliografische Daten sind im Internet über http://dnb.d-nb.de abrufbar.

\section{Springer VS}

(C) Der/die Herausgeber bzw. der/die Autor(en) 2019. Dieses Buch ist eine Open-Access-Publikation.

Open Access Dieses Buch wird unter der Creative Commons Namensnennung 4.0 International Lizenz (http://creativecommons.org/licenses/by/4.0/deed.de) veröffentlicht, welche die Nutzung, Vervielfältigung, Bearbeitung, Verbreitung und Wiedergabe in jeglichem Medium und Format erlaubt, sofern Sie den/die ursprünglichen Autor(en) und die Quelle ordnungsgemäß nennen, einen Link zur Creative Commons Lizenz beifügen und angeben, ob Änderungen vorgenommen wurden.

Die in diesem Buch enthaltenen Bilder und sonstiges Drittmaterial unterliegen ebenfalls der genannten Creative Commons Lizenz, sofern sich aus der Abbildungslegende nichts anderes ergibt. Sofern das betreffende Material nicht unter der genannten Creative Commons Lizenz steht und die betreffende Handlung nicht nach gesetzlichen Vorschriften erlaubt ist, ist für die oben aufgeführten Weiterverwendungen des Materials die Einwilligung des jeweiligen Rechteinhabers einzuholen.

Die Wiedergabe von allgemein beschreibenden Bezeichnungen, Marken, Unternehmensnamen etc. in diesem Werk bedeutet nicht, dass diese frei durch jedermann benutzt werden dürfen. Die Berechtigung zur Benutzung unterliegt, auch ohne gesonderten Hinweis hierzu, den Regeln des Markenrechts. Die Rechte des jeweiligen Zeicheninhabers sind zu beachten.

Der Verlag, die Autoren und die Herausgeber gehen davon aus, dass die Angaben und Informationen in diesem Werk zum Zeitpunkt der Veröffentlichung vollständig und korrekt sind. Weder der Verlag, noch die Autoren oder die Herausgeber übernehmen, ausdrücklich oder implizit, Gewähr für den Inhalt des Werkes, etwaige Fehler oder Äußerungen. Der Verlag bleibt im Hinblick auf geografische Zuordnungen und Gebietsbezeichnungen in veröffentlichten Karten und Institutionsadressen neutral.

Springer VS ist ein Imprint der eingetragenen Gesellschaft Springer Fachmedien Wiesbaden GmbH und ist ein Teil von Springer Nature

Die Anschrift der Gesellschaft ist: Abraham-Lincoln-Str. 46, 65189 Wiesbaden, Germany 


\section{Grußwort}

Liebe Leserinnen und Leser,

wer sich ein langes Leben wünscht, kommt am Älterwerden nicht vorbei. Und doch machen wir uns oft wenig Gedanken darüber. Wie werden wir alt? Hoffentlich gesund! Wie geht es uns dabei? Hoffentlich gut! Der Deutsche Alterssurvey (DEAS) 2017 gibt Antworten auf diese Fragen. Seit mehr als 20 Jahren begleitet die Studie - gefördert vom Bundesfamilienministerium - Teilnehmerinnen und Teilnehmer beim Älterwerden. Wer zu Beginn der Befragung im Jahr 1996 also mit 40 Jahren noch zu den Jüngsten gehörte, ist heute über 60 Jahre alt.

Dieser lange Beobachtungszeitraum erlaubt einen umfassenden Einblick in das Älterwerden von Frauen und Männern. Es zeigt sich: Frauen und Männer altern anders. Frauen übernehmen häufiger und auch früher als Männer Aufgaben in der Pflege von Angehörigen - aber deutlich seltener ein Ehrenamt. Frauen fühlen sich bis zum Alter von 70-80 Jahren seltener einsam und isoliert als Männer; danach jedoch häufiger. Alles eine Frage des Alters? Nicht unbedingt: Denn die festgestellten Unterschiede zwischen Frauen und Männern sind bemerkenswert stabil, also bei den jüngeren und älteren Geburtsjahrgängen gleichermaßen nachweisbar.

Es gibt auch gute Nachrichten: Großväter betreuen ihre Enkelkinder heute oft ähnlich engagiert wie die Großmütter. Jüngere Jahrgänge kommen voraussichtlich gesünder und mit einer höheren Lebenszufriedenheit ins (sehr) hohe Alter und sind häufiger ehrenamtlich engagiert als die vor ihnen geborenen. Und die oft befürchtete „Epidemie der Einsamkeit“ konnte nicht nachgewiesen werden. In allen Altersgruppen fühlen sich weniger als 10 Prozent der Menschen einsam.

So individuell Lebensentwürfe damals wie heute sind: Die Erkenntnisse der DEAS Langzeitstudie liefern uns spannende Einblicke in gesellschaftliche Entwicklungen. Sie bestärken uns, gute Bedingungen zu schaffen, um Sorgetätigkeiten in der Partnerschaft gerecht zu verteilen und die Gleichstellung zwischen Frauen und Männern auch im Alter weiter zu stärken. Sie bestärken uns, dass wir Menschen einladen, ein Ehrenamt zu übernehmen, sich um andere zu kümmern. Dass sie teilhaben an unserer Gesellschaft - und auch ihren Teil geben. Damit tun wir etwas gegen Einsamkeit und für ein zufriedenes und gesundes Altwerden - von Männern und Frauen.

Mit freundlichen Grüßen

Dr. Franziska Giffey

Bundesministerin für Familie, Senioren, Frauen und Jugend 


\section{Vorwort}

Wenn wir älter werden, verändert sich unsere Lebenssituation in vielerlei Hinsicht. In diesem Buch sind wir der Frage nachgegangen, ob und wie sich Gesundheit, psychisches Wohlbefinden, Einsamkeit und soziale Isolation sowie das Engagement in Sorgetätigkeiten und im Ehrenamt über die zweite Lebenshälfte hinweg verändern. Im Zentrum unserer Analysen stand die Frage, ob die Veränderungen, die das Älterwerden mit sich bringt, für Frauen und Männer gleich oder unterschiedlich ausfallen. Da sich die Gesellschaft in den letzten Jahrzehnten gewandelt hat, gehen wir auch der Frage nach, ob sich die Geschlechter im Zuge dieses Wandels angenähert haben. Unsere Ergebnisse zeigen: Zwischen Frauen und Männern zeigen sich viele Unterschiede, aber auch Ähnlichkeiten im Älterwerden. Und obwohl unsere Ergebnisse nahelegen, dass später geborene Jahrgänge im Durchschnitt gesünder und zufriedener das hohe und sehr hohe Alter erreichen werden als früher geborene - und später geborene Jahrgänge seltener einsam sind sowie aktiver in der Familie und der Gesellschaft -, bleiben viele Geschlechterunterschiede im sozialen Wandel bestehen.

Die Analysen zum Älterwerden von Frauen und Männern im sozialen Wandel basieren auf dem Deutschen Alterssurvey (DEAS), der größten Studie zu Alter und Altern in Deutschland. Seit der ersten Erhebung im Jahr 1996 liegen bevölkerungsrepräsentative Angaben zu Frauen und Männern in der zweiten Lebenshälfte vor. Bis zur aktuellsten Erhebung im Jahr 2017 haben über 20000 Menschen am Deutschen Alterssurvey teilgenommen, viele davon über einen längeren Zeitraum von bis zu 21 Jahren. Wir bedanken uns bei allen Teilnehmerinnen und Teilnehmern am Deutschen Alterssurvey sehr herzlich für ihre Auskunftsbereitschaft, ohne ihre Mitarbeit wäre diese Studie nicht möglich gewesen.

Der Deutsche Alterssurvey wird vom Bundesministerium für Familie, Senioren, Frauen und Jugend (BMFSFJ) gefördert. Für ihre umfassende Unterstützung möchten wir uns an dieser Stelle sehr herzlich bedanken bei Matthias von Schwanenflügel, Gabriele Müller-List, Alwin Proost, Annette Pauly, Sabine Ullrich, Sabine Rüger, Martina Fritsch und Michael Janta. Die Datenerhebung des Deutschen Alterssurveys wird durchgeführt von infas Institut für angewandte Sozialwissenschaft, unser Dank gilt Stefan Schiel, Menno Smid, Reiner Gilberg und Jonathan Ruiz Marcos sowie all den Interviewerinnen und Interviewern für ihren Einsatz für den Deutschen Alterssurvey.

Den Mitgliedern des wissenschaftlichen Beirats des Deutschen Alterssurvey danken wir für ihren Rat und ihre hervorragende Unterstützung. Wir danken Christian Alt, Walter Bien, Dorly Deeg, Michael Eid, Markus Grabka, Karsten Hank, Nina Knoll, Susanne Kuger, Klaus Rothermund, Thomas Scharf, Anja Steinbach, Michael Wagner und Susanne Zank.

Unser Dank gilt auch allen Kolleginnen und Kollegen des Deutschen Zentrums für Altersfragen (DZA), die bei der Arbeit am Deutschen Alterssurvey mitgeholfen haben. Wunderbar unterstützt wurden wir von unseren studentischen Mitarbeiterinnen und Mitarbeitern, Constantin Bolz, Tamara Green, Alberto Lozano, Lisa Seidl, Henry Webel 
und Constanze Weber. Ohne die Hilfe von Stefanie Hartmann, Forschungsassistentin am DZA, wäre dieses Buch nie fertig geworden, ihr gilt unser großer Dank für Lektorat, Gestaltung und Organisation. Unterstützt haben zudem Barbara Grönig, Verwaltungsleiterin des DZA, Peter Köster, verantwortlich für die Buchhaltung und Roland Molle, Systemadministrator des DZA. Ein herzliches Dankeschön!
Gemeinsam mit den Autorinnen und Autoren dieses Buches hoffen wir, zur Diskussion, wie und in welcher Gesellschaft wir älter werden wollen und welche Rahmenbedingungen für ein "gutes Altern“ geschaffen oder optimiert werden müssen, beitragen zu können.

Berlin, Dezember 2018

Claudia Vogel, Markus Wettstein und Clemens Tesch-Römer 


\section{Inhalt}

1. Älterwerden von Frauen und Männern in Deutschland _ . . . . . . . . . . . . . 1 Claudia Vogel, Markus Wettstein und Clemens Tesch-Römer

2. Längsschnittliches Design, Inhalte und Methodik des Deutschen Alterssurveys (DEAS)

Daniela Klaus, Heribert Engstler und Claudia Vogel

3. Funktionale und subjektive Gesundheit bei Frauen und Männern im Verlauf der zweiten Lebenshälfte

Svenja M. Spuling, Anja Cengia und Markus Wettstein

4. Lebenszufriedenheit und depressive Symptome bei Frauen und Männern im Verlauf der zweiten Lebenshälfte Markus Wettstein und Svenja M. Spuling

5. Soziale Isolation und Einsamkeit bei Frauen und Männern im Verlauf der zweiten Lebenshälfte

Oliver Huxhold und Heribert Engstler

6. Unbezahlte Sorgetätigkeiten von Frauen und Männern im Verlauf der zweiten Lebenshälfte

Daniela Klaus und Claudia Vogel

7. Ehrenamtliches Engagement von Frauen und Männern im Verlauf der zweiten Lebenshälfte

Claudia Vogel und Laura Romeu Gordo

8. Frauen und Männer im Verlauf der zweiten Lebenshälfte:

Eine Gesamtbetrachtung der empirischen Befunde

Frank Berner, Claudia Vogel, Markus Wettstein und Clemens Tesch-Römer 


\title{
1. Älterwerden von Frauen und Männern in Deutschland
}

\author{
Claudia Vogel, Markus Wettstein und Clemens Tesch-Römer
}

\section{Kernaussagen}

Alternsverläufe und Verlängerung der Lebensspanne: Durch den Anstieg der Lebenserwartung hat sich die Lebensspanne verlängert. Dem Thema, wie sich Alternsverläufe in verschiedenen Lebensbereichen vom mittleren bis ins sehr hohe Erwachsenenalter vollziehen, ist der vorliegende Band gewidmet. Als Datengrundlage für die Analysen von Alternsverläufen eignet sich in besonderer Weise der Deutsche Alterssurvey (DEAS).

Alternsverläufe von Frauen und Männern: Das Geschlecht ist eine zentrale gesellschaftliche Kategorie. Soziale Unterschiede zwischen Frauen und Männern bestehen unter anderem in Bildung, Erwerbsverhalten und Einkommen sowie in unterschiedlichen Geschlechterrollen. Bekannt ist, dass sich Frauen und Männer in vielen Aspekten ihrer Lebenssituationen unterscheiden. Der Frage, ob sich auch Alternsverläufe nach Geschlechterzugehörigkeit unterscheiden, soll im vorliegenden Band mit Blick auf unterschiedliche Lebensbereiche nachgegangen werden.

Sozialer Wandel und Alternsverläufe von Frauen und Männern: Geschlechterunterschiede haben sich in den letzten Jahrzehnten teilweise deutlich, aber nicht in allen Lebensbereichen verändert. Im vorliegenden Bericht wird untersucht, ob sich auch geschlechtsspezifische Alternsverläufe in Deutschland über nachfolgende Geburtsjahrgänge hinweg gewandelt haben. Vorstellbar ist es, dass die Unterschiede in den Alternsverläufen zwischen Frauen und Männern im Zuge des sozialen Wandels in einigen Lebensbereichen kleiner geworden sind, in anderen aber stabil geblieben oder sogar größer geworden sind.

Implikationen für die Politik: Kenntnisse über Alternsverläufe von Frauen und Männern sind notwendig, um wirksame Programme und Maßnahmen für älterwerdende Menschen entwickeln zu können und um Rahmenbedingungen zu schaffen, damit Frauen und Männer gleichermaßen in hoher Lebensqualität alt werden können. 


\subsection{Einleitung}

Die große Mehrheit der Bevölkerung in Deutschland kann ein langes Leben erwarten (Vaupel 2010): Heute beträgt die durchschnittliche Lebenserwartung von Neugeborenen 88 Jahre für Frauen und 84 Jahre für Männer (Statistisches Bundesamt 2018). Für Frauen und Männer, die beispielsweise in den 1930er Jahren geboren sind, war die Lebenserwartung noch deutlich geringer mit 72 Jahren für Frauen und 64 Jahren für Männer (Statistisches Bundesamt 2018). Vor dem Hintergrund der verlängerten Lebensspanne ist es wichtig zu wissen, welche Veränderungen im individuellen Alternsprozess für verschiedene Lebensbereiche auftreten können und ab welchem Punkt im Leben diese im Durchschnitt auftreten.

Es gibt viele Gründe für die Annahme, dass diese individuellen Alternsveränderungen für Frauen und Männer unterschiedlich ausfallen. Die Lebenssituationen unterscheiden sich im mittleren und höheren Lebensalter nach Geschlechterzugehörigkeit in vielfältiger Weise: Frauen verfügen häufiger über eine bessere soziale Einbindung als Männer (z. B. Antonucci, Ajrouch, \& Birditt 2014), sie übernehmen in stärkerem Maß Sorgetätigkeiten als Männer (z. B. Beckmann 2016) und sie sind seltener erwerbstätig (z. B. Simonson, Romeu Gordo, \& Titova 2011). Frauen leiden aber auch häufiger unter depressiven Symptomen (z.B. Sutin, Terracciano, Milaneschi, An, Ferrucci, \& Zonderman 2013; Wolff \& Tesch-Römer 2017) sowie im höheren Alter unter Einbußen der funktionalen Gesundheit als Männer (z. B. Menning \& Hoffmann 2009). Zudem leben ältere Frauen deutlich häufiger allein als ältere Männer (Statistisches Bundesamt 2018). Doch wie stellen sich diese Geschlechterunterschiede im Alternsverlauf dar? Werden die Unterschiede zwischen Frauen und Männern mit zunehmendem Alter größer, bleiben sie stabil, oder werden sie kleiner? Und gilt dies für verschiedene Lebensbereiche (wie Gesundheit, Sorgetätigkeiten oder ehrenamtliches Engagement) gleichermaßen?

Das gesellschaftliche Geschlechterverhältnis hat sich im Verlauf der letzten Jahrzehnte erheb- lich verändert. Die gesellschaftliche Debatte zur Gleichberechtigung hat zu einer Modernisierung der Geschlechterrollen geführt. Starre Geschlechterrollen haben sich teilweise aufgelöst und Möglichkeiten zur gesellschaftlichen Partizipation in der Politik und in der Zivilgesellschaft sind mittlerweile weniger stark an das Geschlecht gebunden als dies noch für frühere Geburtsjahrgänge von Frauen und Männern der Fall war. Gleichzeitig bestehen nach wie vor große geschlechtsspezifische Ungleichheiten, etwa in der Erwerbsbeteiligung und im Erwerbseinkommen. So erzielen Frauen derzeit ein im Verlauf des Lebens erzieltes Gesamteinkommen, das nur etwa die Hälfte des Gesamteinkommens von Männern beträgt (Gender Lifetime Earnings Gap; Deutscher Bundestag 2017). Auch sind die Alterseinkommen von Frauen nach wie vor deutlich geringer als die der Männer (Gender Pension Gap; Grabka, Jotzo, Rasner, \& Westermeier 2017). Trotz der objektiven materiellen Schlechterstellung von Frauen spricht aber einiges dafür, dass die Lebenslagen in vielen Bereichen im sozialen Wandel ähnlicher geworden sind. So scheinen sich Frauen und Männer in den letzten Jahrzehnten beispielsweise hinsichtlich ihres Erlebens von Vereinbarkeitskonflikten zwischen Berufs- und Familienleben angenähert zu haben (Shockley, Shen, DeNunzio, Arvan, \& Knudsen 2017). Auch ist die Diskrepanz zwischen Frauen und Männern hinsichtlich ihrer durchschnittlichen Lebenserwartung kleiner geworden.

Vor dem Hintergrund dieser gesellschaftlichen Veränderungen folgt das vorliegende Buch zwei Leitfragen:

1) Unterscheiden sich Alternsverläufe von Frauen und Männern in Deutschland? Sind die Alternsverläufe von Frauen und Männern in bestimmten Lebensbereichen unterschiedlicher als in anderen?

2) Haben sich geschlechtsspezifische Alternsverläufe in Deutschland über nachfolgende Geburtsjahrgänge hinweg gewandelt? Sind also 
die Unterschiede zwischen Frauen und Männern im Zuge des sozialen Wandels kleiner oder größer geworden - oder sind sie stabil geblieben?

Ziel des vorliegenden Bandes ist es, anhand von empirischen Analysen des Deutschen Alterssurveys (DEAS) Antworten auf diese Fragen zu geben. Die Teilnehmerinnen und Teilnehmer des Deutschen Alterssurveys wurden über einen Zeitraum von bis zu 21 Jahren wiederholt befragt: Die erste Befragung fand im Jahr 1996 statt, die aktuellste Befragung im Jahr 2017. Aufgrund der Anlage des Deutschen Alterssurveys sowie unter Anwendung moderner längsschnittlicher statistischer Analysemethoden ist es möglich, Alternsverläufe über eine große Spanne der zweiten Lebenshälfte zu beschreiben: Analysiert und dargestellt werden geschlechtsspezifische Alternsverläufe von 40 bis 90 Jahren. ${ }^{1}$

Erstens wird analysiert, ob sich Alternsverläufe von Frauen und Männern unterscheiden, und zwar mit Blick auf fünf zentrale Lebensbereiche: funktionale und subjektive Gesundheit, Lebenszufriedenheit und depressive Symptome, soziale Isolation und Einsamkeit, Sorgetätigkeiten und ehrenamtliches Engagement. Zweitens wird empirisch geprüft, ob sich die Unterschiede oder Ähnlichkeiten in den Alternsverläufen zwischen Frauen und Männern über verschiedene Geburtsjahrgänge hinweg gewandelt haben (ebenfalls mit Blick auf die fünf genannten Lebensbereiche). Verglichen werden dabei die Geburtsjahrgänge 1930-1939, 1940-1949 sowie 1950-1959 (eine ausführliche Begründung der Auswahl dieser Geburtsjahrgänge findet sich in Abschnitt 1.3; für detaillierte Informationen zum methodischen Vorgehen siehe Kapitel 2 Design, Inhalte und Methodik des Deutschen

1 Im Deutschen Alterssurvey (DEAS) wurde Geschlecht in der Vergangenheit lediglich binär, also mit den beiden Merkmalsausprägungen „weiblich“ und „männlich“ erfasst. Auf Basis des Deutschen Alterssurveys können somit keine Aussagen über Personen gemacht werden, die sich nicht einem dieser beiden
Alterssurveys in diesem Band). Dabei wird auch die Frage diskutiert, ob die aktuell ,jungen alten“ Frauen und Männer (Neugarten 1974) - sie werden die „alten alten“ Frauen und Männer der Zukunft sein - möglicherweise andere Alternsverläufe erleben werden und ob andere Geschlechterunterschiede in ihren Verläufen zu beobachten sein werden als sie bei jenen Frauen und Männern zu finden sind, die derzeit die Lebensphase des hohen und sehr hohen Alters erleben. Durch den Vergleich der geschlechtsspezifischen Alternsverläufe unterschiedlicher Geburtsjahrgänge (im Folgenden verwenden wir hierfür auch den Begriff der Kohorte) können Aussagen darüber getroffen werden, ob und inwiefern der soziale Wandel eine Veränderung von geschlechtsspezifischen Alternsverläufen mit sich gebracht hat.

Für Gesellschaft und Politik sind Antworten auf diese Fragen von großem Interesse. Aus dem Vergleich der Alternsverläufe geht hervor, ob Alternsveränderungen bei Frauen stärker (oder schwächer) ausfallen als bei Männern und ob diese Veränderungen bei Frauen zu einem früheren (oder späteren) Zeitpunkt einsetzen als bei Männern. Möglicherweise gleichen sich die Alternsverläufe von Frauen und Männern in einigen Lebensbereichen auch, so dass eine geschlechterdifferenzierende Sichtweise nicht notwendig ist. Diese Erkenntnisse sind wichtige Voraussetzungen für die Entwicklung politischer Handlungsoptionen: Für die Bewältigung von alternsbezogenen Herausforderungen könnten in bestimmten Lebensbereichen Maßnahmen und optimierte Rahmenbedingungen in erster Linie für Frauen, in anderen Lebensbereichen für Männer zu entwickeln sein. Es ist aber durchaus möglich, dass sich die Alternsverläufe für Frauen und Männer in bestimmten Lebensbereichen ähneln und dass

Geschlechter zuordnen können. In den allermeisten repräsentativen Studien in Deutschland fehlt bislang die Möglichkeit, neben „weiblich“ und „männlich“ auch die Geschlechtsangabe „divers“ wählen zu können, die im Personenstandsgesetz seit 2018 vorgesehen ist (vgl. Baumann, Egenberger, \& Supik 2018). 
Maßnahmen für Menschen eines bestimmten Alters insgesamt angeboten werden könnten. $\mathrm{Zu}$ dem können aus Alternsverläufen Schlüsse gezogen werden, ob Maßnahmen sich eher an Personen im mittleren Erwachsenenalter, an Personen in der späten Erwerbsphase oder an Personen im (sehr) hohen Alter richten sollten.

\section{2 Älterwerden von Frauen und Männern}

Die Geschlechterzugehörigkeit prägt die Identität und die Lebensentwürfe jedes Menschen ein Leben lang. Die Geschlechterzugehörigkeit wird nicht nur durch biologische Eigenschaften, also genetisch bedingte körperliche Merkmale bestimmt (im Englischen: Sex), sondern auch durch soziale Zuschreibungen und Erwartungen (im Englischen: Gender). Merkmale des sozialen Geschlechts sind zum Beispiel geschlechtsspezifische Rollen, geschlechtertypische Verhaltensweisen und soziale Normen, die häufig auch mit gesellschaftlichen Geschlechterhierarchisierungen einhergehen. Geschlecht ist ein fundamentales gesellschaftliches Ordnungsprinzip, das in allen Lebensbereichen wirksam ist (Backes 2010; Calasanti 2010). Doing Gender beschreibt, dass Geschlecht nicht nur etwas ist, was Menschen haben oder sind, sondern auch etwas, was Menschen selbst tun und wie sie sich Anderen gegenüber verhalten. Beispielsweise sind Verhaltensweisen in sozialen Interaktionen immer davon geprägt, welche Geschlechterzugehörigkeit beim Gegenüber identifiziert wird. Der Prozess des Alterns lässt sich also nicht losgelöst von der Geschlechterzugehörigkeit der alternden Individuen betrachten: Altern verläuft geschlechtsspezifisch (Calasanti \& Slevin 2001; van Dyk 2017).

Der gesamte Lebenslauf wird also durch die Geschlechterzugehörigkeit geprägt, zum Beispiel die Erwerbsbiografie, die Familienbiografie und der Gesundheitsverlauf. Die geschlechtsspezifische Arbeitsteilung in der Familie und in der Gesellschaft beeinflusst, welche Arbeiten und Aufgaben vorrangig von Frauen und welche vorrangig von Männern übernommen werden. Wie prägend die Geschlechterzugehörigkeit für das Leben in Deutschland ist, wird auch dadurch ersicht- lich, dass bestimmte staatliche Regelungen nur für Frauen (z. B. Mutterschutz) oder nur für Männer (z.B. Wehrpflicht) gelten. Gleichzeitig können einheitliche Regulierungen, etwa das Ehegatten-Splitting im Steuerrecht, geschlechterdifferenzierende Konsequenzen - für die Übernahme von Aufgaben wie Sorgetätigkeiten oder Erwerbsarbeit - mit sich bringen (Stichwort: Gender Mainstreaming).

Ein Ziel der Politik ist die Gleichstellung von Männern und Frauen. Im Artikel 3 des Grundgesetzes heißt es: „Männer und Frauen sind gleichberechtigt. Der Staat fördert die tatsächliche Durchsetzung der Gleichberechtigung von Frauen und Männern und wirkt auf die Beseitigung bestehender Nachteile hin“. Deshalb ist die Identifikation von Geschlechterunterschieden in Alternsveränderungen sowie deren Entwicklung im sozialen Wandel unabdingbar, um Gleichstellungspolitik sowie weitere Maßnahmen zu betreiben, um Frauen und Männern gleichermaßen zu einem „guten“ und „erfolgreichen Altern“ (Rowe \& Kahn 1997) zu verhelfen.

In diesem Band beschäftigen wir uns mit Alternsveränderungen. Der Begriff des Älterwerdens bezieht sich auf Veränderungsprozesse, die Individuen mit zunehmendem Alter erleben. Prozesse der Entwicklung und des Älterwerdens beginnen nicht erst mit dem Übergang in die Lebensphase (hohes) Alter, sondern finden über die gesamte Lebensspanne statt, und zwar von Geburt an (Baltes, Lindenberger, \& Staudinger 2006). Daher ist dieser Prozess auch keinesfalls mit einem bestimmten Lebensalter abgeschlossen. Auch im höheren und hohen Alter, also mit 60, 70 oder 80 Jahren und später können sich erhebliche Veränderungen in vielen Lebensbereichen ereignen. Altern ist 
multidimensional, betrifft also unterschiedliche Lebensbereiche, und Altern ist multidirektional, kann also Gewinne in einigen Bereichen und Verluste in anderen Bereichen umfassen (vgl. Baltes et al. 2006).

Unterschiede im Älterwerden von Frauen und Männern können etwa in den Bereichen der geschlechtsspezifischen, ungleichen Erwerbs- und Familienbiografien sowie der ungleichen Gesundheitsverläufe bestehen, weshalb eine Lebenslaufperspektive für die Gleichstellungspolitik als unerlässlich erachtet wird (vgl. den Ersten Gleichstellungsbericht mit dem Titel „Neue Wege - Gleiche Chancen: Gleichstellung von Frauen und Männern im Lebensverlauf “ Deutscher Bundestag 2011). Geschlechterunterschiede können bedingt sein durch eine traditionelle Arbeitsteilung bezüglich der Übernahme bezahlter Erwerbsarbeit und unbezahlter Fürsorgearbeit und durch ungleichen Zugang zu Ressourcen, etwa aufgrund von Geschlechterunterschieden in Bildung und Einkommen. Zudem können Unterschiede in Alternsveränderungen zwischen Frauen und Männern Auswirkungen von Geschlechterstereotypen, Geschlechterdiskriminierung und Rollenbildern (möglicherweise überlagert von Altersbildern und Altersdiskriminierung) sowie von geschlechtsspezifisch verschiedenen Restriktionen, Präferenzen und biologischen Faktoren sein.

Darüber hinaus sind die Lebensläufe in modernen Wohlfahrtstaaten wie Deutschland institutionell gerahmt, so dass bestimmte Lebenslaufpassagen in der dreigeteilten Normalbiografie Schule-Erwerbsleben-Ruhestand für alle Individuen erwartbar sind (vgl. zur Institutionalisierung des Lebenslaufs Kohli 1985). Zum Beispiel prägt die Altersgrenze zum Erhalt einer Altersrente aus der gesetzlichen Rentenversicherung die individuellen Erwerbsbiografien der großen Mehrheit der in Deutschland lebenden Menschen (vgl. Clemens 2010). Gleichzeitig trägt eine erwerbsarbeitszentrierte Sozialpolitik dazu bei, geschlechtsspezifisch unterschiedliche Alterseinkommen $\mathrm{zu}$ generieren, je nachdem, ob die individuelle Erwerbsbiografie dem Lebenslaufmuster "Normalbiografie“ mit kontinuierlicher Vollzeitbeschäftigung folgt oder nicht. Meier-Gräwe (2010) zeigt auf, dass geschlechtsspezifische Unterschiede entlang von Lebensläufen insbesondere beim Übergang zur Elternschaft entstehen, wenn sich die Frage der Vereinbarkeit von Sorge- und Erwerbsarbeit stellt, die in der Regel von Müttern und Vätern unterschiedlich beantwortet wird. Vor dem Hintergrund bestehender Rahmenbedingungen und Restriktionen auszuhandelnde Entscheidungen, etwa darüber, ob Frauen und Männer kontinuierlich und in Vollzeit oder diskontinuierlich einer Erwerbstätigkeit nachgehen, wirken sich über den gesamten Lebenslauf bis ins hohe Alter aus. Gleichzeitig wird mit dem Eintritt in den Ruhestand als Beginn der Lebensphase Alter häufig die Erwartung einer Angleichung der Lebenssituationen von Frauen und Männern verknüpft, das Alter wird somit als "Gleichmacher" nach der Beendigung der Erwerbsarbeit angesehen (vgl. Gildemeister 2008). Obwohl bekannt ist, dass Frauen und Männer nicht unter den gleichen Bedingungen in die Lebensphase Alter eintreten, zum Beispiel aufgrund der genannten sozialen Ungleichheiten in der Übernahme der Erwerbs- und Fürsorgearbeit, steht eine Überprüfung dieser Angleichungsthese anhand des Vergleichs von Alternsverläufen jedoch noch weitgehend aus.

Geschlechterunterschiede in allen Phasen des Lebenslaufes können einen aus gleichstellungspolitischer Sicht weichenstellenden Charakter haben für eine Gleichstellung auch im höheren Lebensalter. Beispiele für Geschlechterunterschiede, die sich auf die individuellen geschlechtsspezifischen Alternsprozesse auswirken können, gibt es aus verschiedenen Lebensbereichen. Beispielsweise sind mehr Frauen als Männer von funktionalen Einschränkungen betroffen, also von Einschränkungen bei Alltagstätigkeiten wie Treppensteigen (Wolff, Nowossadeck, \& Spuling 2017). Dies könnte eine mögliche Erklärung dafür sein, dass Frauen in einigen Aspekten des psychischen Wohlbefindens niedrigere Werte aufweisen als Männer: Ältere Frauen berichten im Durchschnitt eine geringere Lebenszufriedenheit als ältere Männer (Pinquart 
\& Sörensen 2001), und zumindest in bestimmten Altersphasen sind Frauen häufiger von depressiven Symptomen betroffen als Männer (Sutin et al. 2013). Auch Einsamkeit tritt gemäß einiger Studien (z. B. Luhmann \& Hawkley 2016) häufiger bei Frauen auf als bei Männern; allerdings gibt es auch Befunde, wonach sich Frauen und Männer nicht hinsichtlich ihrer Einsamkeit unterscheiden (z. B. Böger, Wetzel, \& Huxhold 2017). Vorliegende Geschlechterunterschiede im psychischen Wohlbefinden und im Einsamkeitserleben können - neben gesundheitlichen Faktoren - viele Ursachen haben, etwa die bereits genannten Geschlechterunterschiede in der Übernahme von Sorgetätigkeiten oder Unterschiede in Bildung und Einkommen.

Eine zusätzliche Perspektive ergibt sich dann, wenn sowohl Geschlecht als auch Alter als Dimensionen sozialer Ungleichheit betrachtet werden. Wie bereits ausgeführt, geht Ungleichheit zwischen Frauen und Männern häufig mit anderen Aspekten von Benachteiligungen wie etwa der Berufsoder der Einkommensposition einher. Aber auch das Lebensalter ist eine wichtige Dimension sozialer Ungleichheit: Mit zunehmendem Alter werden Menschen - aufgrund ihres Alters - nicht selten benachteiligt oder sogar diskriminiert (Ayalon \& Tesch-Römer 2018). Beispielsweise haben ältere Menschen häufig einen eingeschränkten Zugang zu Leistungen des Gesundheitssystems (Remmers 2012). Überlappen sich verschiedene Merkmale sozialer Ungleichheit, so wird in der sozialwissenschaftlichen Forschung häufig der Begriff der Intersektionalität verwendet (Carbado, Crenshaw, Mays, \& Tomlinson 2013). Eine ungleichheitswirksame Intersektionalität der sozialen Kategorien Geschlecht und Alter liegt beispielsweise dann vor, wenn ältere Frauen (für diese Gruppe treffen die Überlappung der Merkmalsausprägungen „Frau“ und "hohes Alter“ zu) besonders stark unter Benachteiligungen leiden, während dies für Männer (ältere und jüngere) sowie für jüngere Frauen nicht der Fall ist.

Die Theorie kumulativer Nachteile nimmt an, dass soziale Ungleichheiten im Verlauf des Lebens größer werden (Dannefer 2003; Ferraro \& Mor- ton 2016). Möglicherweise gibt es auch, je nach betrachtetem Lebensbereich, eine kumulative Ungleichheit zwischen Frauen und Männern, bedingt durch die andauernde (und im Verlauf des Lebens sich kumulierende) Ungleichbehandlung von Frauen und Männern, etwa in Familie, Kindergarten, Schule, Ausbildung, Berufstätigkeit und Sorgetätigkeit. Ungleichheit (zwischen Frauen und Männern) ist also keineswegs eine stabile Gegebenheit, sondern entfaltet sich im Lebenslauf (TeschRömer 2018) und kann sich kumulativ verstärken.

Aufgrund dieser Überlegungen werden wir im vorliegenden Band in jedem der untersuchten Lebensbereiche den Fragen nachgehen, ob und wie sich im Verlauf des Älterwerdens Ungleichheiten zwischen Frauen und Männer verändern. Wie bereits erörtert, sind, je nach betrachtetem Lebensbereich, verschiedene Szenarien denkbar: Unterschiede zwischen Frauen und Männern können sich mit dem Älterwerden, etwa durch Prozesse kumulativer Ungleichheit verstärken - Ungleichheiten, die im mittleren Erwachsenenalter zwischen Frauen und Männern bestehen, können im höheren Alter aber auch stabil bleiben oder sich sogar verringern. 


\section{3 Älterwerden im sozialen Wandel}

Zudem werden wir der Frage nachgehen, ob und wie sich die geschlechtsspezifischen Alternsverläufe über verschiedene Lebensbereiche in einander nachfolgenden Geburtsjahrgängen unterscheiden. Gerade mit Blick auf den gesellschaftlichen $\mathrm{Zu}$ sammenhalt ist die Frage von zentraler Bedeutung, ob Geschlechterunterschiede in Alternsprozessen über den Verlauf der historischen Zeit kleiner oder größer werden.

Unterschiedliche Geburtsjahrgänge können Altern und Alter unterschiedlich erfahren. Unsere Gesellschaft ist von einem fortwährenden sozialen Wandel geprägt. Gesellschaftliche Modernisierung und kultureller Wandel, technischer und medizinischer Fortschritt, verbesserte Bildungsbedingungen sowie Trends zunehmender Detraditionalisierung und Individualisierung sind Treiber dieses Wandels (vgl. z. B. Beck 1992; Hellas, Lash, \& Morris 1996; Schaie, Willis, \& Pennak 2005). In westlichen Gesellschaften gibt es zudem einen Trend hin zu einer höheren Flexibilität und Freiheit in der Gestaltung des persönlichen Lebens und sozialer Beziehungen (Allan 2008). Daher ist anzunehmen, dass Menschen heute anders altern und altern werden als etwa die Geburtsjahrgänge ihrer Eltern oder Großeltern.

In diesem Band werden wir die Alternsverläufe dreier ausgewählter Geburtskohorten miteinander vergleichen. Für diese Geburtsjahrgänge liegen im ausreichenden Umfang längsschnittliche Beobachtungen im Deutschen Alterssurvey (DEAS) vor, die eine zuverlässige separate Schätzung von Alternsverläufen ermöglichen (siehe Kapitel $2 \mathrm{De}$ sign, Inhalte und Methodik des Deutschen Alterssurveys in diesem Band). Hierbei handelt es sich um die Jahrgänge der 1930 bis 1939, 1940 bis 1949 sowie 1950 bis 1959 Geborenen. Diese Geburtsjahrgänge wuchsen unter ganz unterschiedlichen Bedingungen auf, etwa hinsichtlich des technischen Fortschritts oder der wirtschaftlichen Situation (und den damit verknüpften Chancen des beruflichen Fortkommens). Zudem gab es im Zeitraum von 1930 bis 2017 einschneidende Ereignisse, die ent- weder nur bestimmte Jahrgänge überhaupt erlebt haben (etwa den Zweiten Weltkrieg), oder die diese Jahrgänge in einem unterschiedlichen Alter erlebt haben (etwa die deutsche Wiedervereinigung), weswegen sich diese Ereignisse unterschiedlich auf die Geburtskohorten ausgewirkt haben könnten. Auch die Sozialversicherungssysteme - Rentenversicherung, Krankenversicherung, Arbeitslosenversicherung, Pflegeversicherung - haben sich für unterschiedliche Jahrgänge ganz verschieden gestaltet. Sozialversicherungssysteme wirken auf Erwerbs- und Familienbiografien und das Älterwerden und prägen somit „Verwirklichungschancen“, die Frauen und Männer im Lebenslauf haben (Helfferich 2008: 32).

Der medizinische Fortschritt mag Menschen späterer Geburtsjahrgänge im Vergleich zu früheren Geburtskohorten zu einer besseren Gesundheit im Alter und einer verkürzten Phase der in Krankheit verbrachten Lebensjahre verholfen haben (Fries 2005), und möglicherweise haben Frauen und Männer unterschiedlich von medizinischen Entwicklungen profitiert (so scheint - beispielsweise in den USA - die Geschlechterdiskrepanz in der Lebenserwartung etwas geringer geworden zu sein; Crimmins \& Beltrán-Sánchez 2011). Allerdings sind die bisher identifizierten Kohortentrends bei gesundheitlichen Faktoren, auch der subjektiven Gesundheit (König, Drewelies, Norman, Spira, Buchmann, Hülür, Eibich, Wagner, Lindenberger, Steinhagen-Thiessen, Gerstorf, \& Demuth 2018), keineswegs durchgängig positiv (Crimmins \& Beltrán-Sánchez 2011), und die letzte Lebensphase später geborener Kohorten ist nicht zwingend von geringeren Rückgängen in Wohlbefinden und kognitiver Leistung geprägt als die früher geborener Kohorten (Gerstorf, Ram, Hoppmann, Willis, \& Schaie 2011; Hülür, Infurna, Ram, \& Gerstorf 2013; Hülür, Ram, \& Gerstorf 2015).

In den vergangenen Jahrzehnten haben sich auch die gesellschaftliche Wahrnehmung von Geschlecht und Alter erheblich verändert. Geschlechterstereotype und Geschlechterrollen haben sich 
in den letzten Jahrzehnten stark gewandelt (Ostner 2005). Gesellschaftliche Altersbilder (Bundesministerium für Familie, Senioren, Frauen und Jugend 2010), die das individuelle Älterwerden sowie die Einstellungen zum eigenen Älterwerden maßgeblich prägen, haben sich im Zeitverlauf geändert (Beyer, Wurm, \& Wolff 2017; Ng, Allore, Trentalange, Monin, \& Levy 2015), was dazu führen könnte, dass Frauen und Männer heute in anderer Weise altern als die Geburtsjahrgänge vor ihnen. Damit könnten sich Geschlechterunterschiede in Alternsprozessen für bestimmte Lebensbereiche verkleinert haben.

Die Auswirkungen des sozialen Wandels sind also offenbar komplex. Sie können sich je nach Lebensalter der unterschiedlichen Geburtsjahrgänge ganz anders auswirken - ältere Menschen (die Angehörigen früher geborener Geburtsjahrgänge) sind davon in anderer Weise betroffen als jüngere Menschen (die Angehörigen später geborener Geburtsjahrgänge). Für Gesellschaft und Politik ist es von großer Bedeutung, etwas über die „Generationenschicksale“ (Kaufmann 1993) unterschiedlicher Geburtsjahrgänge sowie die sich mit der historischen Zeit verändernden Alternsprozesse von Frauen und Männern einander nachfolgender Geburtsjahrgänge zu erfahren. Im Folgenden seien einige Stichpunkte für gesellschaftliche Veränderungen genannt, welche die drei betrachteten Geburtsjahrgänge erlebt haben:

- Geburtsjahrgänge 1930 bis 1939: Die in dieser Zeit geborenen Personen wurden in Kindheit und Jugend während der Zeit des Nationalsozialismus sozialisiert und erlebten einen Großteil ihrer Kindheit und Jugendjahre zur Zeit des Zweiten Weltkriegs. Im frühen Erwachsenenalter waren Angehörige dieser Geburtsjahrgänge die Träger des „Wirtschaftswunders“. Geschlechterrollen und Geschlechterungleichheiten waren in diesen Geburtsjahrgängen deutlich traditionell ausgeprägt. So waren Frauen, die in den 1930er Jahren geboren wurden, zu Beginn ihrer Erwerbsbiografien noch gesetzlich auf die Zustimmung ihres Ehemannes angewie- sen, wenn sie einen Arbeitsvertrag abschließen wollten. Erst seit 1958 durften Ehefrauen in der Bundesrepublik selbst über eine Erwerbstätigkeit entscheiden. Allerdings entspricht auch die damalige gesetzliche Regelung nicht den heutigen Vorstellungen der Gleichberechtigung zwischen den Geschlechtern. So hieß es im Gleichberechtigungsgesetz ( $\$ 1356)$ : „Die Frau führt den Haushalt in eigener Verantwortung. Sie ist berechtigt, erwerbstätig zu sein, soweit dies mit ihren Pflichten in Ehe und Familie vereinbar ist."

- Geburtsjahrgänge 1940 bis 1949: Viele Personen, die in den 1940er Jahren geboren wurden, haben die 68er Jahre als prägend erlebt, eine Zeit, in der durch den kulturellen Wandel und die sexuelle Revolution auch die gesellschaftlichen Geschlechterverhältnisse eine Modernisierung erfahren haben. Die Erwerbs- und Familienbiografien dieser Geburtsjahrgänge sind durch veränderte sozialpolitische Regelungen und den wirtschaftlichen Aufschwung im Nachkriegsdeutschland anders geprägt worden als die Erwerbs- und Familienbiografien früher geborener Geburtsjahrgänge. Die Modernisierung der Geschlechterverhältnisse haben die Angehörigen dieser Geburtsjahrgänge miterlebt oder zum Teil sogar persönlich vorangetrieben.

- Geburtsjahrgänge 1950 bis 1959: Die Jahrgänge der 1950 bis 1959 Geborenen sind bereits zu einem Teil der Babyboomer-Generation zuzurechnen. Ihre Lebenssituation hat sich im Vergleich zu den früher geborenen Kohorten der 1930 bis 1949 Geborenen in vielerlei Hinsicht anders gestaltet. Beispielsweise profitierten sie bereits von der Bildungsexpansion, so dass ein deutlich größerer Anteil insbesondere von Frauen, aber auch von Männern höhere Bildungsabschlüsse erreichte. Die erreichten Bildungsabschlüsse von Frauen und Männern glichen sich daher in diesen Geburtsjahrgängen stark an. Darüber hinaus waren in den 1950er geborene Frauen seltener Hausfrauen und zu höheren Anteilen erwerbstätig als die früher geborenen Frauen. 
In bestimmten Lebensbereichen könnten sich daher die Alternsveränderungen von Frauen und Männern angeglichen haben, wenn man sie für die drei Geburtskohorten vergleicht. Für andere Lebensbereiche dagegen könnten geschlechtsspezifische Verlaufsunterschiede auch stabil geblieben oder sogar größer geworden sein. Nicht zuletzt mag es Lebensbereiche geben, in denen sich - unabhängig von der betrachteten Geburtskohorte keine bedeutsamen Geschlechterunterschiede in den Alternsverläufen manifestieren.

\section{4 Älterwerden in zentralen Lebensbereichen}

Je nach Lebensbereich können Prozesse des Älterwerdens sehr unterschiedlich ausfallen (vgl. z. B. Naegele 2010; Baltes et al. 2006). Wir untersuchen in diesem Band fünf Kernbereiche, die eine hohe Relevanz für die Lebensqualität haben und die bedeutsamen Veränderungen im Lauf des Erwachsenenalters und im Verlauf der zweiten Lebenshälfte bis hinein in das höhere und hohe Lebensalters unterliegen: funktionale und subjektive Gesundheit, Lebenszufriedenheit und depressive Symptome, soziale Isolation und Einsamkeit, Sorgetätigkeiten, ehrenamtliches Engagement. Alle diese im Folgenden beschriebenen Lebensbereiche sind biografisch miteinander verknüpft. Auch die Alternsveränderungen in diesen Bereichen erfolgen nicht notwendigerweise unabhängig voneinander, sondern unter wechselseitiger Beeinflussung.

a) Funktionale und subjektive Gesundheit: Die Gesundheit ist in allen Lebensphasen wichtig. Mit zunehmendem Alter gewinnt sie aber möglicherweise noch weiter an Bedeutung (Böhm, Tesch-Römer, \& Ziese 2009). In der gerontologischen Forschung wird Gesundheit als eine zentrale Komponente „erfolgreichen Alterns“ verstanden (Rowe \& Kahn 1997). Verschiedene Aspekte der körperlichen Gesundheit verändern sich über die zweite Lebenshälfte hinweg in unterschiedlicher Weise (Spuling, Wiest, Huxhold, \& Wurm 2013). Zudem können Veränderungsdynamiken der Gesundheit im mittleren Erwachsenenalter anders ausfallen als etwa im höheren und (sehr) hohen Alter (Wett- stein \& Wahl 2018). Diese gesundheitlichen Veränderungen haben bedeutsame Konsequenzen für die selbstständige Lebensführung und Lebensqualität von Frauen und Männern in der zweiten Lebenshälfte.

b) Lebenszufriedenheit und depressive Symptome: Ein weiterer zentraler Lebensbereich, der eng vernetzt ist mit Aspekten der körperlichen Gesundheit, ist der Bereich der Lebenszufriedenheit und der depressiven Symptomatik. Wie gut es uns geht und wie zufrieden wir mit unserem Leben sind, ist nicht in erster Linie eine Frage des chronologischen Alters (Herschbach 2002; Staudinger 2000), dennoch lassen sich bestimmte Veränderungstrends des psychischen Wohlbefindens über den Verlauf der zweiten Lebenshälfte hinweg beobachten. Gerade das hohe und sehr hohe Alter stellt hierbei eine verletzliche Lebensphase dar, in der bei einem beträchtlichen Anteil an Personen ein Zuwachs depressiver Symptome zu beobachten ist (Diegelmann, Schilling, \& Wahl 2016; Schilling, Wahl, \& Reidick 2013).

c) Soziale Isolation und Einsamkeit: Menschen jeden Alters haben das Bedürfnis, gute und enge Beziehungen $\mathrm{zu}$ anderen Menschen zu haben, die ihnen Zugehörigkeit und Geborgenheit vermitteln (Baumeister \& Leary 1995). Haben sie keine solchen engen Beziehungen, sind sie sozial isoliert und fühlen sich einsam (Hawthorne 2006). Einsam fühlen sich Menschen aber nicht nur bei fehlenden sozialen Beziehungen, sondern auch, wenn die Qualität des persönlichen Austauschs mit anderen Menschen nicht die 
zentralen Bedürfnissen nach Vertrautheit, $\mathrm{Zu}$ gehörigkeit und Geborgenheit erfüllt (Perlman \& Peplau 1981). Einsamkeit und soziale Isolation sind keine Phänomene, die nur im höheren Alter vorkommen. Auch jüngere Menschen sind davon betroffen (Böger \& Huxhold 2018). Wie sich die Risiken, sozial isoliert zu sein und sich einsam zu fühlen, mit dem Älterwerden im Verlauf der zweiten Lebenshälfte verändern, ist empirisch noch nicht ausreichend geklärt. Dies $\mathrm{zu}$ untersuchen ist auch deshalb wichtig, weil insbesondere längere Phasen der Einsamkeit die Lebensqualität beeinträchtigen und gesundheitsgefährdend sind (Luo, Hawkley, Waite, \& Cacioppo 2012).

d) Sorgetätigkeiten: Sorgetätigkeiten und Unterstützungsleistungen, die man für andere Menschen innerhalb (und außerhalb) der Familie übernimmt oder von anderen erhält, sind für den Zusammenhalt und die Pflege von sozialen Beziehungen von großer Bedeutung (vgl. z.B. Klaus \& Tesch-Römer 2017; Mahne \& Klaus 2017). Werden etwa Angehörige wie die eigenen Eltern, die Lebenspartnerin oder der Lebenspartner pflegebedürftig, oder werden Enkelkinder geboren, stellen sich Frauen und Männern mit dem Älterwerden neue Betreuungs- und Unterstützungsaufgaben (vgl. den Zweiten Bericht zur Gleichstellung von Frauen und Männern mit dem Titel „Erwerbs- und Sorgearbeit gemeinsam neu gestalten“, Deutscher Bundestag 2017).

e) Ehrenamtliches Engagement: Ehrenamtliches Engagement ist eine wichtige Form der gesellschaftlichen Partizipation. Im höheren Lebensalter, wenn die Erwerbspartizipation in den Hintergrund rückt, erfolgt die gesellschaftliche Partizipation verstärkt über die Übernahme eines Ehrenamtes. Möglicherweise müssen ehrenamtliche Tätigkeiten im Verlauf des Älterwerdens aber auch aufgegeben werden, etwa aufgrund von Höchstaltersgrenzen im Ehrenamt oder aufgrund gesundheitlicher Einschränkungen (Wetzel \& Simonson 2017; Müller \& Tesch-Römer 2017). Mit dem Älterwerden verändern sich also die individuellen Gelegenheitsstrukturen zur sozialen Teilhabe.

Das vorliegende Buch ist wie folgt aufgebaut: Daten und Methoden werden vorgestellt, auf denen die in diesem Buch berichteten Befunde beruhen. Danach folgen fünf thematische Kapitel mit empirischen Ergebnissen zu Alternsverläufen von Frauen und Männern, gegliedert nach den oben eingeführten zentralen Lebensbereichen. In einem abschließenden Kapitel wird ein Fazit der vorgelegten Befunde gezogen.

Wir haben diesen Band für Personen geschrieben, die sich für das Alter und das Älterwerden, für Geschlechterunterschiede und Gleichstellung oder für Seniorenpolitik und für Gleichstellungspolitik interessieren. Es ist notwendig, mehr über Verläufe des Älterwerdens sowie Geschlechterunterschiede in diesen Verläufen zu erfahren, um wirksame Programme und Maßnahmen für älterwerdende Menschen entwickeln zu können und um Rahmenbedingungen zu schaffen, damit Frauen und Männer gleichermaßen „erfolgreich altern“ (Rowe \& Kahn 1997; Tesch-Römer \& Wahl 2017) und möglichst "gut" ins hohe und sehr hohe Alter kommen. Wir wollen dabei das Augenmerk darauf lenken, dass es in einigen Politikfeldern durchaus sinnvoll sein kann, zukünftig unterschiedliche Maßnahmen für älterwerdende Frauen und für älterwerdende Männer zu konzipieren. Die in diesem Band vorgelegten Befunde geben Aufschluss darüber, in welchen Lebensbereichen es bedeutsame Alternsveränderungen im Leben von Frauen und Männer heute gibt - und welche Veränderungen in $\mathrm{Zu}$ kunft voraussichtlich zu erwarten sind, wenn die in der Vergangenheit beobachteten Trends anhalten. Dieses Wissen ist unverzichtbar für seniorenpolitische und für gleichstellungspolitische Entscheidungen. 


\section{Literatur}

Allan, G. (2008). Flexibility, friendship, and family. Personal Relationships, 15(1), 1-16. doi: 10.1111/ j.1475-6811.2007.00181.x.

Antonucci, T. C., Ajrouch, K. J., \& Birditt, K. S. (2014). The convoy model: explaining social relations from a multidisciplinary perspective. The Gerontologist, 54(1), 82-92.

Ayalon, L., \& Tesch-Römer, C. (Hrsg.). (2018). Contemporary perspectives on ageism. Cham: Springer International Publishing.

Backes, G. M. (2010). Alter(n) - ein kaum entdecktes Arbeitsfeld in der Frauen- und Geschlechterforschung. In: R. Becker \& B. Kortendiek (Hrsg.) Handbuch Frauen- und Geschlechterforschung: Theorie, Methoden, Empirie (S. 454-460). Wiesbaden: VS Verlag für Sozialwissenschaften.

Baltes, P. B., Lindenberger, U., \& Staudinger, U. M. (2006). Life Span Theory in Developmental Psychology. In: W. Damon \& R. M. Lerner (Hrsg.) Handbook of Child Psychology (6 Aufl., Bd. 1, S. 569-664). New York: Wiley.

Baumann, A.-L., Egenberger, V., \& Supik, L. (2018). Erhebung von Antidiskriminierungsdaten in repräsentativen Wiederholungsbefragungen. Bestandsaufnahme und Entwicklungsmöglichkeiten. Berlin: Antidiskriminierungsstelle des Bundes.

Baumeister, R. F., \& Leary, M. R. (1995). The need to belong - desire for interpersonal attachment as a fundamental human-motivation. Psychological Bulletin, 117(3), 497-529. doi: 10.1037/0033-2909. 117.3.497.

Beck, U. (1992). Risk Society. London: SAGE Publications.

Beckmann, S. (2016). Sorgearbeit (Care) und Gender. In: J. Block, C. Hagen \& F. Berner (Hrsg.) Expertisen zum Siebten Altenbericht der Bundesregierung. Berlin: Deutsches Zentrum für Altersfragen.

Beyer, A.-K., Wurm, S., \& Wolff, J. (2017). Älter werden - Gewinn oder Verlust? Individuelle Altersbilder und Altersdiskriminierung. In: K. Mahne, J. K. Wolff, J. Simonson \& C. Tesch-Römer (Hrsg.) Altern im Wandel. Zwei Jahrzehnte Deutscher Alterssurvey (DEAS) (S. 329-343). Wiesbaden: Springer VS.

Böger, A., \& Huxhold, O. (2018). Age-Related Changes in Emotional Qualities of the Social Network From Middle Adulthood Into Old Age: How Do They Relate to the Experience of Loneliness? Psychology and Aging, 33(3), 482-496. doi: 10.1037/pag0000222.

Böger, A., Wetzel, M., \& Huxhold, O. (2017). Allein unter vielen oder zusammen ausgeschlossen: Einsamkeit und wahrgenommene soziale Exklusion in der zweiten Lebenshälfte. In: K. Mahne, J. K. Wolff, J. Simonson \& C. Tesch-Römer (Hrsg.) Altern im Wandel. Zwei Jahrzehnte Deutscher Alterssurvey (DEAS) (S. 273-285). Wiesbaden: Springer VS.

Böhm, K., Tesch-Römer, C., \& Ziese, T. (Hrsg.). (2009). Gesundheit und Krankheit im Alter [Beiträge zur Gesundheitsberichterstattung des Bundes]. Berlin: Robert Koch-Institut.

Bundesministerium für Familie, Senioren, Frauen und Jugend (2010). Eine neue Kultur des Alterns. Altersbilder in der Gesellschaft - Erkenntnisse und Empfehlungen des Sechsten Altenberichts. Berlin: Bundesministerium für Familie, Senioren, Frauen und Jugend.

Calasanti, T. M. (2010). Gender and Ageing in the Context of Globalization. In: D. Dannefer \& C. Phillipson (Hrsg.) The Sage Handbook of Social Gerontology (S. 137-149). Los Angeles: SAGE Publications.

Calasanti, T. M., \& Slevin, K. F. (2001). Gender, Social Inequalities and Aging. Berkeley: AltaMira Press.

Carbado, D. W., Crenshaw, K. W., Mays, V. M., \& Tomlinson, B. (2013). Intersectionality: Mapping the movements of a theory. Du Bois Review: Social Science Research on Race, 10(2), 303-312. 
Clemens, W. (2010). Lebensläufe im Wandel - Gesellschaftliche und sozialpolitische Perspektiven. In: G. Naegele (Hrsg.) Soziale Lebenslaufpolitik (S. 86-109). Wiesbaden: VS Verlag für Sozialwissenschaften.

Crimmins, E. M., \& Beltrán-Sánchez, H. (2011). Mortality and Morbidity Trends: Is There Compression of Morbidity? The Journals of Gerontology, Series B: Psychological Sciences and Social Sciences, 66B(1), 75-86.

Dannefer, D. (2003). Cumulative advantage/disadvantage and the life course. Cross-fertilizing age and social science theory. The Journals of Gerontology, Series B: Psychological Sciences and Social Sciences, 58(6), 327-337.

Deutscher Bundestag (2017). Zweiter Gleichstellungsbericht der Bundesregierung. Erwerbs- und Sorgearbeit gemeinsam neu gestalten. (Drucksache 18/12840). Berlin: Deutscher Bundestag.

Deutscher Bundestag (2011). Erster Gleichstellungsbericht. Neue Wege-Gleiche Chancen. Gleichstellung von Frauen und Männern im Lebensverlauf. (Drucksache 17/6240). Berlin: Deutscher Bundestag.

Diegelmann, M., Schilling, O. K., \& Wahl, H.-W. (2016). Feeling blue at the end of life: Trajectories of depressive symptoms from a distance-to-death perspective. Psychology and Aging, 31(7), 672-686. doi: 10.1037/pag0000114.

Dyk, S. van (2017). Zur Interdependenz und Analyse von Alter(n) und Geschlecht. Theoretische Erkundungen und zeitdiagnostische Überlegungen. In: T. Denninger \& L. Schütze (Hrsg.) Alter(n) und Geschlecht. Neuverhandlung eines sozialen Zusammenhangs (S. 24-50). Münster: Westfälisches Dampfboot.

Ferraro, K. F., \& Morton, P. M. (2016). What do we mean by accumulation? Advancing conceptual precision for a core idea in gerontology. The Journals of Gerontology, Series B: Psychological Sciences and Social Sciences, 73(2), 269-278.

Fries, J. F. (2005). The Compression of Morbidity. The Milbank Quarterly, 83(4), 801-823.

Gerstorf, D., Ram, N., Hoppmann, C., Willis, S. L., \& Schaie, K. W. (2011). Cohort Differences in Cognitive Aging and Terminal Decline in the Seattle Longitudinal Study. Developmental Psychology, 47(4), 10261041.

Gildemeister, R. (2008). Was wird aus der Geschlechterdifferenz im Alter? Über die Angleichung von Lebensformen und das Ringen um biografische Kontinuität. In: S. Buchen \& M. S. Maier (Hrsg.) Älterwerden neu denken (S. 197-215). Wiesbaden: VS Verlag für Sozialwissenschaften.

Grabka, M. M., Jotzo, B., Rasner, A., \& Westermeier, C. (2017). Der Gender Pension Gap verstärkt die Einkommensungleichheit von Männern und Frauen im Rentenalter. DIW Wochenbericht, 84(5), 87-96.

Hawthorne, G. (2006). Measuring Social Isolation in Older Adults: Development and Initial Validation of the Friendship Scale. Social Indicators Research, 77(3), 521-548. doi: 10.1007/s11205-005-7746-y.

Helfferich, C. (2008). Alles beim Alten? Wie der demografische Wandel Lebenslaufmuster von Frauen und Männern morgen und das Alter übermorgen beeinflusst. In: S. Buchen \& M. S. Maier (Hrsg.) Älterwerden neu denken (S. 31-45). Wiesbaden: VS Verlag für Sozialwissenschaften.

Hellas, P., Lash, S., \& Morris, P. (1996). Detraditionalization. Oxford: Blackwell.

Herschbach, P. (2002). Das „Zufriedenheitsparadox“ in der Lebensqualitätsforschung. Psychotherapie. Psychosomatik. Medizinische Psychologie, 52(03/04), 141-150. doi: 10.1055/s-2002-24953.

Hülür, G., Infurna, F. J., Ram, N., \& Gerstorf, D. (2013). Cohorts based on decade of death: No evidence for secular trends favoring later cohorts in cognitive aging and terminal decline in the AHEAD study. Psychology and Aging, 28(1), 115-127.

Hülür, G., Ram, N., \& Gerstorf, D. (2015). Historical improvements in well-being do not hold in late life: Birth- and death-year cohorts in the United States and Germany. Developmental Psychology, 51(7), 998-1012. doi: 10.1037/a0039349. 
Kaufmann, F.-X. (1993). Generationenbeziehungen und Generationenverhältnisse im Wohlfahrtsstaat. In: K. Lüscher \& F. Schultheis (Hrsg.) Generationenbeziehungen in „postmodernen“ Gesellschaften (S. 95108). Konstanz: Universitätsverlag Konstanz.

Klaus, D., \& Tesch-Römer, C. (2017). Pflege und Unterstützung bei gesundheitlichen Einschränkungen: Welchen Beitrag leisten Personen in der zweiten Lebenshälfte für andere? In: K. Mahne, J. K. Wolff, J. Simonson \& C. Tesch-Römer (Hrsg.) Altern im Wandel. Zwei Jahrzehnte Deutscher Alterssurvey (DEAS) (S. 185-200). Wiesbaden: Springer VS.

Kohli, M. (1985). Die Institutionalisierung des Lebenslaufs: Historische Befunde und theoretische Argumente. Kölner Zeitschrift für Soziologie und Sozialpsychologie, 37(1), 1-29.

König, M., Drewelies, J., Norman, K., Spira, D., Buchmann, N., Hülür, G., Eibich, P., Wagner, G. G., Lindenberger, U., Steinhagen-Thiessen, E., Gerstorf, D., \& Demuth, I. (2018). Historical trends in modifiable indicators of cardiovascular health and self-rated health among older adults: Cohort differences over 20 years between the Berlin Aging Study (BASE) and the Berlin Aging Study II (BASE-II). PLoS one, 13(1), e0191699. doi: 10.1371/journal.pone.0191699.

Luhmann, M., \& Hawkley, L. (2016). Age differences in loneliness from late adolescence to oldest old age. Developmental Psychology, 52(6), 943-959.

Luo, Y., Hawkley, L. C., Waite, L. J., \& Cacioppo, J. T. (2012). Loneliness, health, and mortality in old age: A national longitudinal study. Social Science \& Medicine, 74(6), 907-914. doi: 10.1016/j.socsci med.2011.11.028.

Mahne, K., \& Klaus, D. (2017). Zwischen Enkelglück und (Groß-)Elternpflicht - die Bedeutung und Ausgestaltung von Beziehungen zwischen Großeltern und Enkelkindern. In: K. Mahne, J. K. Wolff, J. Simonson \& C. Tesch-Römer (Hrsg.) Altern im Wandel. Zwei Jahrzehnte Deutscher Alterssurvey (DEAS) (S. 231-245). Wiesbaden: Springer VS.

Meier-Gräwe, U. (2010). Erwerbsarbeit und generative Sorgearbeit neu bewerten und anders verteilen - Perspektiven einer gendersensiblen Lebenslaufpolitik in modernen Dienstleistungsgesellschaften. In: G. Naegele (Hrsg.) Soziale Lebenslaufpolitik (S. 245-267). Wiesbaden: VS Verlag für Sozialwissenschaften.

Menning, S., \& Hoffmann, E. (2009). Funktionale Gesundheit und Pflegebedürftigkeit. Gesundheit und Krankheit im Alter. In: K. Böhm, C. Tesch-Römer \& T. Ziese (Hrsg.) Gesundheit und Krankheit im Alter [Beiträge zur Gesundheitsberichterstattung des Bundes] (S. 62-78). Berlin: Robert Koch-Institut.

Müller, D., \& Tesch-Römer, C. (2017). Früheres Engagement und Engagementbereitschaft. In: J. Simonson, C. Vogel \& C. Tesch-Römer (Hrsg.) Freiwilliges Engagement in Deutschland. Der Deutsche Freiwilligensurvey 2014 (S. 153-178). Wiesbaden: Springer VS.

Naegele, G. (Hrsg.). (2010). Soziale Lebenslaufpolitik. Wiesbaden: VS Verlag für Sozialwissenschaften.

Neugarten, B. L. (1974). Age Groups in American Society and the Rise of the Young-Old. The Annals of the American Academy of Political and Social Science, 415(1), 187-198. doi: 10.1177/000271627441500114.

Ng, R., Allore, H. G., Trentalange, M., Monin, J. K., \& Levy, B. R. (2015). Increasing Negativity of Age Stereotypes across 200 Years: Evidence from a Database of 400 Million Words. PLOS ONE, 10(2), e0117086.

Ostner, I. (2005). Einführung: Wandel der Geschlechtsrollen - Blickpunkt Väter: eine vernachlässigte Kategorie in sozialwissenschaftlicher Theorie und Empirie. Zeitschrift für Familienforschung, 17(1), 46-49.

Perlman, D., \& Peplau, L. A. (1981). Toward a social psychology of loneliness. In: R. Gilmour \& S. Duck (Hrsg.) Personal Relationships: 3. Relationships in Disorder (S. 31-56). London: Academic Press.

Pinquart, M., \& Sörensen, S. (2001). Gender differences in self-concept and psychological well-being in old age: a meta-analysis. The Journals of Gerontology, Series B: Psychological Sciences and Social Sciences, 56(4), 195-213. 
Remmers, H. (2012). Rationierung und Altersdiskriminierung. In: F. Berner, J. Rossow \& K.-P. Schwitzer (Hrsg.) Altersbilder in der Wirtschaft, im Gesundheitswesen und in der pflegerischen Versorgung: Expertisen zum Sechsten Altenbericht der Bundesregierung (S. 339-368). Wiesbaden: VS Verlag für Sozialwissenschaften.

Rowe, J. W., \& Kahn, R. L. (1997). Successful Aging. The Gerontologist, 37(4), 433-440.

Schaie, K. W., Willis, S. L., \& Pennak, S. (2005). An Historical Framework for Cohort Differences in Intelligence. Research in Human Development, 2(1-2), 43-67.

Schilling, O. K., Wahl, H.-W., \& Reidick, O. (2013). Trajectories of depressive symptoms in advanced old age: A functional approach concerning the role of physical functioning. GeroPsych: The Journal of Gerontopsychology and Geriatric Psychiatry, 26(1), 29-38. doi: 10.1024/1662-9647/a000079.

Shockley, K. M., Shen, W., DeNunzio, M. M., Arvan, M. L., \& Knudsen, E. A. (2017). Disentangling the relationship between gender and work-family conflict: An integration of theoretical perspectives using meta-analytic methods. Journal of Applied Psychology, 102(12), 1601-1635.

Simonson, J., Romeu Gordo, L., \& Titova, N. (2011). Changing employment patterns of women in Germany: How do baby boomers differ from older cohorts? A comparison using sequence analysis. Advances in Life Course Research, 16(2), 65-82.

Spuling, S. M., Wiest, M., Huxhold, O., \& Wurm, S. (2013). Bildungsabhängige Verläufe verschiedener Dimensionen der Gesundheit. Berlin: Deutsches Zentrum für Altersfragen.

Statistisches Bundesamt (2018). Bevölkerung und Erwerbstätigkeit. Haushalte und Familien. Ergebnisse des Mikrozensus. Fachserie 1 Reihe 3. Wiesbaden: Statistisches Bundesamt.

Staudinger, U. M. (2000). Viele Gründe sprechen dagegen, und trotzdem geht es vielen Menschen gut: Das Paradox des subjektiven Wohlbefindens. Psychologische Rundschau, 51(4), 185-197.

Sutin, A. R., Terracciano, A., Milaneschi, Y., An, Y., Ferrucci, L., \& Zonderman, A. B. (2013). The trajectory of depressive symptoms across the adult lifespan. JAMA psychiatry, 70(8), 803-811. doi: 10.1001/ja mapsychiatry.2013.193.

Tesch-Römer, C. (2018). Theorien der sozial- und verhaltenswissenschaftlichen Alternsforschung. In: K. Hank, F. Schulz-Nieswandt, M. Wagner \& S. Zank (Hrsg.) Alternsforschung. Handbuch für Wissenschaft und Praxis (S. 49-82). Baden-Baden: Nomos.

Tesch-Römer, C., \& Wahl, H.-W. (2017). Toward a More Comprehensive Concept of Successful Aging: Disability and Care Needs. The Journals of Gerontology, Series B: Psychological Sciences and Social Sciences, 72 (2), 310-318. doi: https://doi.org/10.1093/geronb/gbw162.

Vaupel, J. W. (2010). Biodemography of human ageing. Nature, 464(7288), 536-542. doi: 10.1038/natu re08984-542.

Wettstein, M., \& Wahl, H.-W. (2018). Höheres Alter. In: C.-W. Kohlmann, C. Salewski \& M. A. Wirtz (Hrsg.) Psychologie in der Gesundheitsförderung (S. 605-616). Bern: Hogrefe.

Wetzel, M., \& Simonson, J. (2017). Engagiert bis ins hohe Alter? Organisationsgebundenes ehrenamtliches Engagement in der zweiten Lebenshälfte. In: K. Mahne, J. K. Wolff, J. Simonson \& C. Tesch-Römer (Hrsg.) Altern im Wandel. Zwei Jahrzehnte Deutscher Alterssurvey (DEAS) (S. 81-95). Wiesbaden: Springer VS.

Wolff, J. K., Nowossadeck, S., \& Spuling, S. M. (2017). Altern nachfolgende Kohorten gesünder? Selbstberichtete Erkrankungen und funktionale Gesundheit im Kohortenvergleich. In: K. Mahne, J. K. Wolff, J. Simonson \& C. Tesch-Römer (Hrsg.) Altern im Wandel. Zwei Jahrzehnte Deutscher Alterssurvey (DEAS) (S. 125-138). Wiesbaden: Springer VS. 
Wolff, J. K., \& Tesch-Römer, C. (2017). Glücklich bis ins hohe Alter? Lebenszufriedenheit und depressive Symptome in der zweiten Lebenshälfte. In: K. Mahne, J. K. Wolff, J. Simonson \& C. Tesch-Römer (Hrsg.) Altern im Wandel. Zwei Jahrzehnte Deutscher Alterssurvey (DEAS) (S. 171-183). Wiesbaden: Springer VS.

Open Access Dieses Kapitel wird unter der Creative Commons Namensnennung 4.0 International Lizenz (http:// creativecommons.org/licenses/by/4.0/deed.de) veröffentlicht, welche die Nutzung, Vervielfältigung, Bearbeitung, Verbreitung und Wiedergabe in jeglichem Medium und Format erlaubt, sofern Sie den/die ursprünglichen Autor(en) und die Quelle ordnungsgemäß nennen, einen Link zur Creative Commons Lizenz beifügen und angeben, ob Änderungen vorgenommen wurden.

Die in diesem Kapitel enthaltenen Bilder und sonstiges Drittmaterial unterliegen ebenfalls der genannten Creative Commons Lizenz, sofern sich aus der Abbildungslegende nichts anderes ergibt. Sofern das betreffende Material nicht unter der genannten Creative Commons Lizenz steht und die betreffende Handlung nicht nach gesetzlichen Vorschriften erlaubt ist, ist für die oben aufgeführten Weiterverwendungen des Materials die Einwilligung des jeweiligen Rechteinhabers einzuholen. 


\title{
2. Längsschnittliches Design, Inhalte und Methodik des Deutschen Alterssurveys (DEAS)
}

\author{
Daniela Klaus, Heribert Engstler und Claudia Vogel
}

\section{Kernaussagen}

Deutscher Alterssurvey (DEAS): Der Deutsche Alterssurvey ist eine repräsentative Langzeitstudie zu Lebenssituationen und Alternsverläufen von Frauen und Männern in Deutschland ab 40 Jahren. Zwischen 1996 und 2017 wurden sechs Erhebungswellen realisiert (1996, 2002, 2008, 2011, 2014, 2017).

Stichproben: Bislang haben 20129 Personen an der Studie teilgenommen, für die 39446 Befragungen vorliegen. Für 49 Prozent der Teilnehmerinnen und Teilnehmer liegen Wiederholungsbefragungen vor. Die Stichproben bestehen aus etwa gleich vielen Frauen und Männern und umfassen einen Zeitraum bis zu 21 Jahren.

Verläufe bis ins hohe Alter: Die Längsschnittstichproben eignen sich in besonderer Weise zur Untersuchung der Alternsverläufe von Frauen und Männern über die Altersspanne von 40 bis 90 Jahren: Gründe dafür sind die hohen Fallzahlen, die gestiegene Wiederteilnahmebereitschaft der Teilnehmerinnen und Teilnehmer, der lange Beobachtungszeitraum und die vergleichsweise geringe Panelselektivität.

Befragungsformen: Um besondere Befragtengruppen besser berücksichtigen zu können, wurden im DEAS 2017 die Befragungsformen erweitert. Im Fall gesundheitsbedingter Nichtteilnahme der Befragungsperson konnten Interviews mit einer Stellvertreterperson geführt werden (Proxy-Interviews). Außerdem konnte der schriftliche Selbstausfüller-Fragebogen entweder als Papierfragebogen ausgefüllt werden oder als Online-Fragebogen.

Alternsrelevante Themen: Das Erhebungsinstrument deckt eine große Breite alternsrelevanter Themen ab. Neben der Erwerbsarbeit und dem Ruhestand sind dies unter anderem Gesundheit und Gesundheitsverhalten, Hilfe- und Pflegebedürftigkeit, Lebensqualität und Wohlbefinden, Partnerschaft, Familie und Generationenbeziehungen, das soziale Netzwerk und Unterstützung, sowie ehrenamtliches Engagement. 


\subsection{Einleitung}

Der Deutsche Alterssurvey (DEAS) ist eine vom Bundesministerium für Familie, Senioren, Frauen und Jugend (BMFSFJ) geförderte interdisziplinäre Langzeitstudie des Deutschen Zentrums für Altersfragen (DZA) zu den Lebenssituationen und Alternsverläufen der Menschen in der zweiten Lebenshälfte in Deutschland. Zielsetzungen sind sowohl eine differenzierte Dauerbeobachtung als Sachgrundlage für Politik und Gesellschaft (Sozialberichterstattung) als auch die sozial- und verhaltenswissenschaftliche Erforschung individueller Alternsverläufe, sozialer Unterschiede und deren Veränderungen im sozialen Wandel. Zudem dient der DEAS der Erweiterung der Dateninfrastruktur durch Bereitstellung umfassender repräsentativer Mikrodaten für die Wissenschaft. ${ }^{1}$ Die Teilnehmenden dieser Langzeitstudie werden in persönlich-mündlichen Interviews und einem ergänzenden Selbstausfüller-Fragebogen wiederholt und umfassend zu ihren objektiven und subjektiven Lebensumständen befragt. Dabei zeichnet sich die Studie durch eine große Themenbreite sowie eine interdisziplinäre Anlage aus (vgl. weiterführend Abschnitt 3).

Dem Deutschen Alterssurvey liegt ein kohortensequenzielles Stichprobendesign zu Grunde: Alle sechs Jahre wird eine neue Querschnittstichprobe 40 - bis 85 -Jähriger aus dem Einwohnermelderegister gezogen (Basisstichproben), die jeweils als Längsschnittstichproben fortgeführt werden (vgl. weiterführend Abschnitt 2). Mit der jüngsten DEAS-Erhebung im Jahr 2017 hat sich der Beobachtungszeitraum der Studie auf 21 Jahre und sechs Erhebungswellen erweitert (1996, 2002, 2008, 2011, 2014, 2017). Insgesamt stehen 39446 Inter-

1 Die aufbereiteten und anonymisierten Befragungsdaten aller abgeschlossenen Erhebungswellen des Deutschen Alterssurveys (DEAS) sowie die dazugehörige Datendokumentation werden vom Forschungsdatenzentrum des Deutschen Zentrums für Altersfragen (DZA) als Scientific Use Files für wissenschaftliche Sekundäranalysen kostenlos zur Verfügung gestellt (siehe www.fdz-dza.de). views mit 20129 Personen für Analysen zur Verfügung. ${ }^{2}$ Die Stichprobenziehung und -pflege sowie die Feldarbeit erfolgen seit Studienbeginn durch das infas Institut für angewandte Sozialwissenschaft.

In den bisher veröffentlichten Ergebnisbänden des Deutschen Zentrums für Altersfragen zu den einzelnen Erhebungswellen des Deutschen Alterssurveys stand die querschnittliche Untersuchung der Lebenssituationen der Menschen in der zweiten Lebenshälfte und des sozialen Wandels ihrer Lebensumstände im Vordergrund (Mahne, Wolff, Simonson, \& Tesch-Römer 2017; Motel-Klingebiel, Wurm, \& Tesch-Römer 2010; Tesch-Römer, Engstler, \& Wurm 2006). Diese Bände stützten sich ausschließlich auf die Informationen aus dem ersten Interview der Studienteilnehmerinnen und -teilnehmer, das heißt die Basisstichproben der Jahre 1996, 2002, 2008 und 2014. Im Mittelpunkt des vorliegenden Bandes steht die Untersuchung der individuellen Entwicklungen im Alternsverlauf. Hierfür werden neben den Informationen aus dem Basisinterview einer Person vor allem auch die Informationen aus den Wiederholungsbefragungen darauffolgender Jahre genutzt. Angesichts der langen Studiendauer nimmt eine wachsende Zahl der Befragten bis in ihr zehntes Lebensjahrzehnt hinein am Deutschen Alterssurvey teil. Auf dieser Datengrundlage werden zu ausgewählten Themen die Alternsverläufe vom 40. bis zum 90. Lebensjahr untersucht, also vom mittleren Erwachsenenalter bis in die Hochaltrigkeit (vgl. weiterführend zur Auswertungsstrategie dieses Bandes Abschnitt 4).

2 Zuzüglich einer einmaligen Stichprobe unter der ausländischen Bevölkerung in Deutschland aus dem Jahr 2002 ( $n=586)$, deren Teilnehmerinnen und Teilnehmer allerdings nicht für eine Wiederbefragung vorgesehen waren. 


\subsection{Stichprobendesign}

Abbildung 2-1 illustriert das Stichprobendesign des Deutschen Alterssurveys (DEAS), das sich als Kombination von quer- und längsschnittlichen Stichproben darstellt. Seit 1996 wird alle sechs Jahre eine Einwohnermeldeamtsstichprobe aus der in Privathaushalten lebenden Bevölkerung jener Geburtsjahrgänge gezogen, die im jeweiligen Befragungsjahr ihr 40. bis 85. Lebensjahr vollenden. Bislang liegen vier unabhängige Querschnittstichproben für die Jahre 1996, 2002, 2008 und 2014 vor. Für 2020 ist die Ziehung und Befragung der nächsten Stichprobe geplant. Diese Basisstichproben bilden die Grundlage für die Längsschnittstichproben, indem die Studienteilnehmerinnen und -teilnehmer im Laufe ihres individuellen Älterwerdens mehrfach interviewt werden. Die Daten dieser Längsschnittstichproben geben Auskunft über individuelle Alternsveränderungen, beispielsweise in der Gesundheit, den sozialen Beziehungen oder im psychischen Wohlbefinden. Da jede Basisstichprobe in den Längsschnitt überführt wird, liegen im Deutschen Alterssurvey für unterschiedliche Geburtsjahrgänge Informationen zu individuellen Veränderungen in vergleichbaren Alternsabschnitten vor. Auf Basis dieses sogenannten kohortensequenziellen Stichprobendesigns lässt sich untersuchen, ob sich die Alternsverläufe von Personen aus unterschiedlichen Geburtskohorten unterscheiden.

\section{Querschnittstichproben}

Ausgangspunkt für die Wiederholungsbefragungen (Längsschnittstichproben) sind die vier Basisbefragungen (Querschnittstichproben) aus den Jahren 1996, 2002, 2008 und 2014. Dabei handelt es sich um Repräsentativbefragungen der in

Abbildung 2-1 Stichprobendesign des Deutschen Alterssurveys (DEAS), 1996-2017

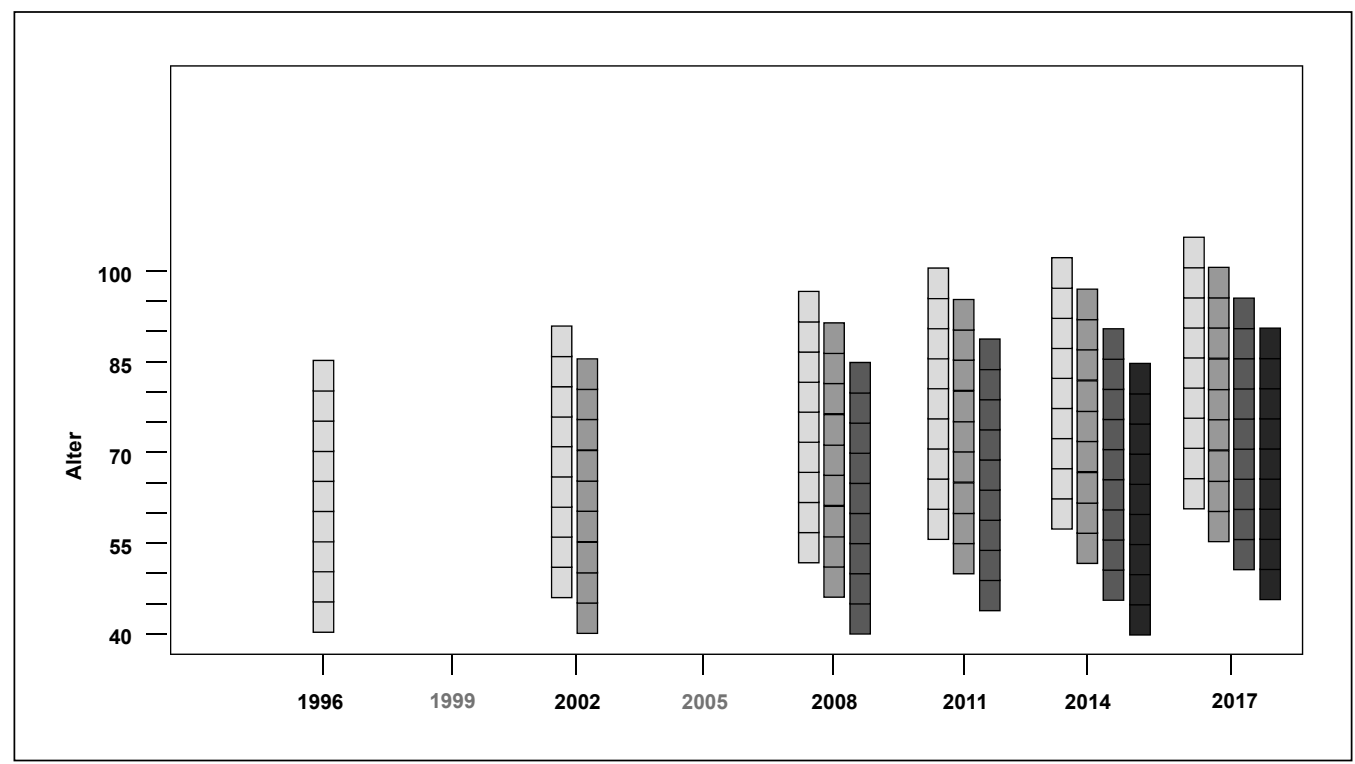

Quelle: Deutsches Zentrum für Altersfragen (DZA). Deutscher Alterssurvey (DEAS). 
Deutschland lebenden Wohnbevölkerung ${ }^{3}$ zwischen 40 und 85 Jahren in Privathaushalten. ${ }^{4}$

Diese Befragungen gehen aus einer zweistufigen Stichprobenziehung hervor: Auf eine bundesweite Ziehung von 290 Gemeinden (nach dem Probability-Proportional-to-Size-Ansatz) folgt eine zufällige Personenstichprobe, die aus den Einwohnermelderegistern dieser Gemeinden gezogen wird. Diese Stichproben sind nach Alter, Geschlecht und Region (wohnhaft in Ost- und Westdeutschland) geschichtet: Höhere Altersgruppen, Männer und Personen aus Ostdeutschland sind überproportional vertreten, um sicherzustellen, dass die Fallzahlen auch für Personengruppen mit geringen Bevölkerungsanteilen in der älteren Bevölkerung ausreichend groß sind, um detaillierte Analysen durchführen zu können. So legt dieses Vorgehen beispielsweise die Grundlage dafür, dass auch die Alternsverläufe von Männern trotz ihrer im Vergleich zu Frauen geringeren Lebenserwartung mit ausreichender Fallzahl bis ins hohe Alter beobachtet werden können.

In den vier Querschnittstichproben wurden folgende Fallzahlen realisiert, wobei eine rückläufige Teilnahmebereitschaft ${ }^{5}$ deutlich wird, die auch aus anderen Umfragestudien bekannt ist: 4838 (50 Prozent) in der Welle 1996, 3084 (38 Prozent) in der Welle 2002, 6205 (36 Prozent) in der Welle 2008, 6002 (27 Prozent) in der Welle 2014. Nach dem mündlichen Interview werden die Befragten gebeten, ergänzend einen schriftlichen Fragebogen auszufüllen, was regelmäßig von einem Großteil der Befragten getan wird. Allerdings ist auch hier ein Rückgang der Teilnahme ${ }^{6}$ festzustellen: 4034 (83 Prozent der Personen, die am mündli-

3 Ab 2008 erfolgt die Ziehung unabhängig von der deutschen Staatsangehörigkeit. In den Jahren 1996 und 2002 wurden die Stichproben aus der deutschen Wohnbevölkerung gezogen.

4 Bei Wiederholungsbefragungen werden zwar prinzipiell auch Teilnehmerinnen und Teilnehmer, die in der Zwischenzeit in ein Heim umgezogen sind, befragt. Allerdings führen der bei Heimbewohnerinnen und -bewohnern oft sehr schlechte Gesundheitszustand und Probleme des Zugangs zur Zielperson zu chen Interview 1996 teilgenommen haben), 2787 (90 Prozent im Jahr 2002), 4442 (72 Prozent im Jahr 2008), 4350 (72 Prozent im Jahr 2014). Dabei unterscheidet sich die Teilnahmebereitschaft zwischen Frauen und Männern kaum.

Um die disproportionale Schichtung der Stichprobenziehung sowie die nicht-repräsentative Teilnahme bestimmter Bevölkerungsgruppen auszugleichen, werden Querschnittgewichte gebildet. Diese stellen eine Randanpassung der realisierten Stichprobe an die amtliche Bevölkerungsstatistik (Mikrozensus) in Bezug auf die drei Schichtungsmerkmale dar: Geschlecht, Altersgruppe (40-54, 55-69, 70-85 Jahre) und Region (Ost- und Westdeutschland). Solche Gewichte liegen getrennt für die mündlichen sowie schriftlichen Befragungsteile vor. Ein Abgleich von gewichteten Verteilungen zentraler sozio-demografischer Merkmale zwischen dem Deutschen Alterssurvey und der amtlichen Statistik belegen eine gute Repräsentativität - abgesehen von leichten Abweichungen, die auch aus anderen Studien bekannt sind. So sind im Deutschen Alterssurvey gegenüber der Verteilung in der Bevölkerung Familienhaushalte und Befragte aus der Mittelschicht etwas überrepräsentiert, Befragte mit Migrationshintergrund sind unterrepräsentiert, und verstärkt im hohen Alter zeigt sich eine Unterrepräsentation von Pflegebedürftigen und Personen mit Schwerbehinderung. Insgesamt deckt der Deutsche Alterssurvey mit diesen vier Erstbefragungen eine breite Spanne von Geburtskohorten ab, nämlich Personen, die zwischen 1911 und 1974 geboren sind (für detaillierte Informationen zu den Querschnittstichproben siehe Klaus \& Engstler 2017: 33-39).

einer sehr geringen Wiederbefragungsteilnahme unter ihnen. Von den im Jahr 2017 erneut befragten Personen im Alter von 43 bis 97 Jahren waren weniger als ein Prozent Heimbewohnerinnen und -bewohner.

5 In Klammern wird die Teilnahmequote berichtet. Diese errechnet sich als der Anteil gültiger mündlicher Interviews an der bereinigten Bruttoeinsatzstichprobe.

6 Anteil der schriftlichen Fragebögen an der Anzahl der gültigen mündlichen Interviews. 


\section{Längsschnittstichproben}

Seit 2002 werden Wiederholungsbefragungen durchgeführt: zunächst im Sechsjahresrhythmus und seit $2008 \mathrm{im}$ Abstand von drei Jahren. In Tabelle 2-1 sind die realisierten Längsschnittstichproben beschrieben, getrennt nach Befragungsjahr und Jahr der Erstbefragung. Für Wiederbefragungen sind grundsätzlich alle Erstteilnehmerinnen und -teilnehmer vorgesehen. Allerdings ist gemäß datenschutzrechtlicher Bestimmungen die Vorbedingung für eine wiederholte Kontaktaufnahme das Vorliegen einer schriftlichen Einverständniserklärung der Studienteilnehmerinnen und -teilnehmer, deren Kontaktdaten speichern zu dürfen. Um diese Erklärung wird während der Erstbefragung gebeten. Diese sogenannte Panelbereitschaft ist über die Querschnittstichproben hinweg deutlich angestiegen: Haben sich 1996 nur wenig mehr als die Hälfte mit einer Datenspeicherung einverstanden erklärt (56 Prozent), waren es 2014 bereits 76 Prozent. Dieser Anstieg in Verbindung mit der Taktungsverkürzung zwischen den Panelwellen auf drei Jahre hat zu einer Zunahme der Wiederteilnahmequoten geführt: Für 9834 Personen beziehungsweise 49 Prozent aller Studienteilnehmerinnen und -teilnehmer liegen Längsschnittdaten mit mindestens zwei Teilnahmen vor: 4990 Erstbefragte haben zweimal teilgenommen (25 Prozent), 1530 dreimal (8 Prozent), 2299 viermal (11 Prozent), 705 fünfmal (3 Prozent) und für 310 Erstbefragte liegen sogar insgesamt sechs Teilnahmen vor (2 Prozent).

Abbildung 2-2 illustriert die Anteile gültiger Wiederholungsbefragungen an der Anzahl der Befragungen im jeweiligen Erstbefragungsjahr. Im Verlauf der Studie kam es zu einer Abmilderung der hohen Ausfälle zwischen Erst- und Zweitbefragung: Während beispielsweise von den 2002 erstmals Befragten sechs Jahre später (2008) nur 32 Prozent erneut für die Studie gewonnen werden konnten, liegt der vergleichbare Anteil für im Jahr 2008 Erstbefragte drei Jahre später (2011) mit 46 Prozent deutlich darüber. Diese positive Ent-

\section{Abbildung 2-2 Wiederbefragungsquoten 1996-2017}

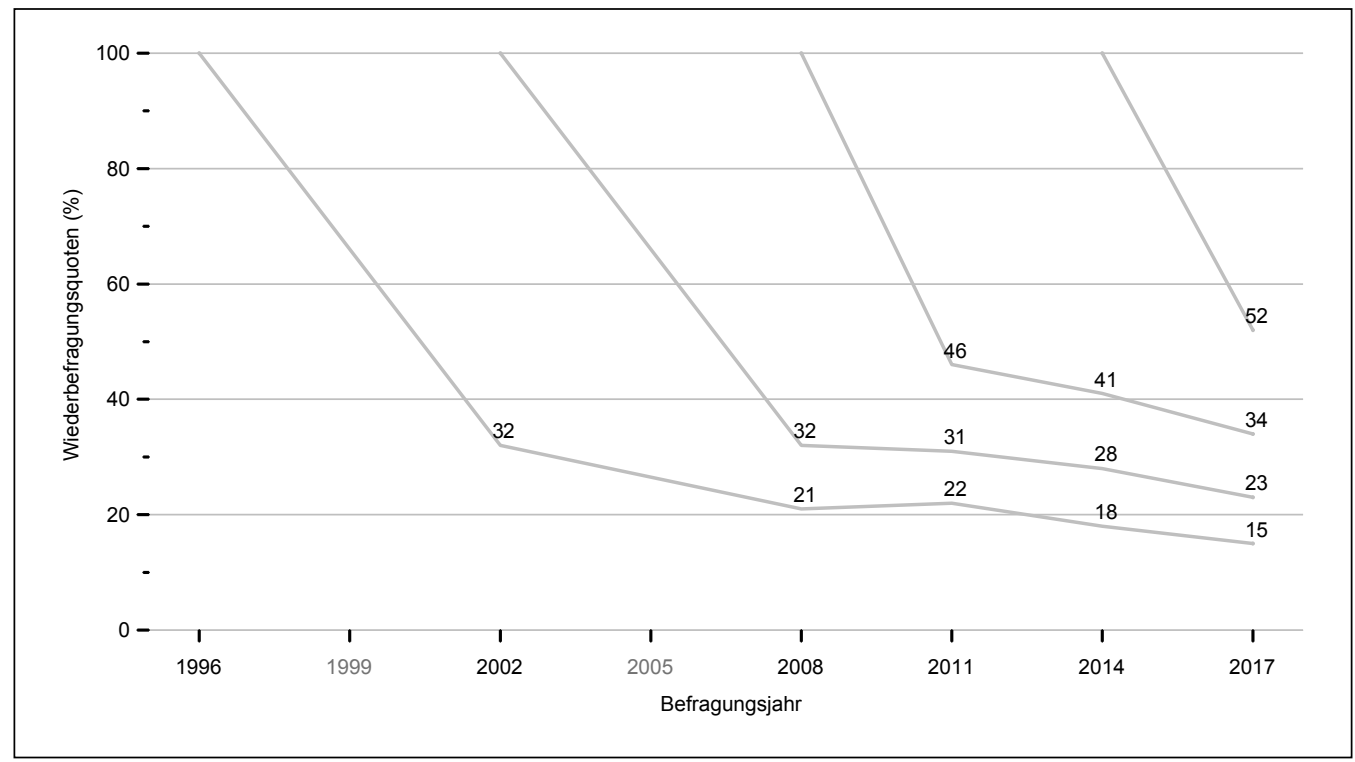

Quelle: DEAS 1996-2017. 


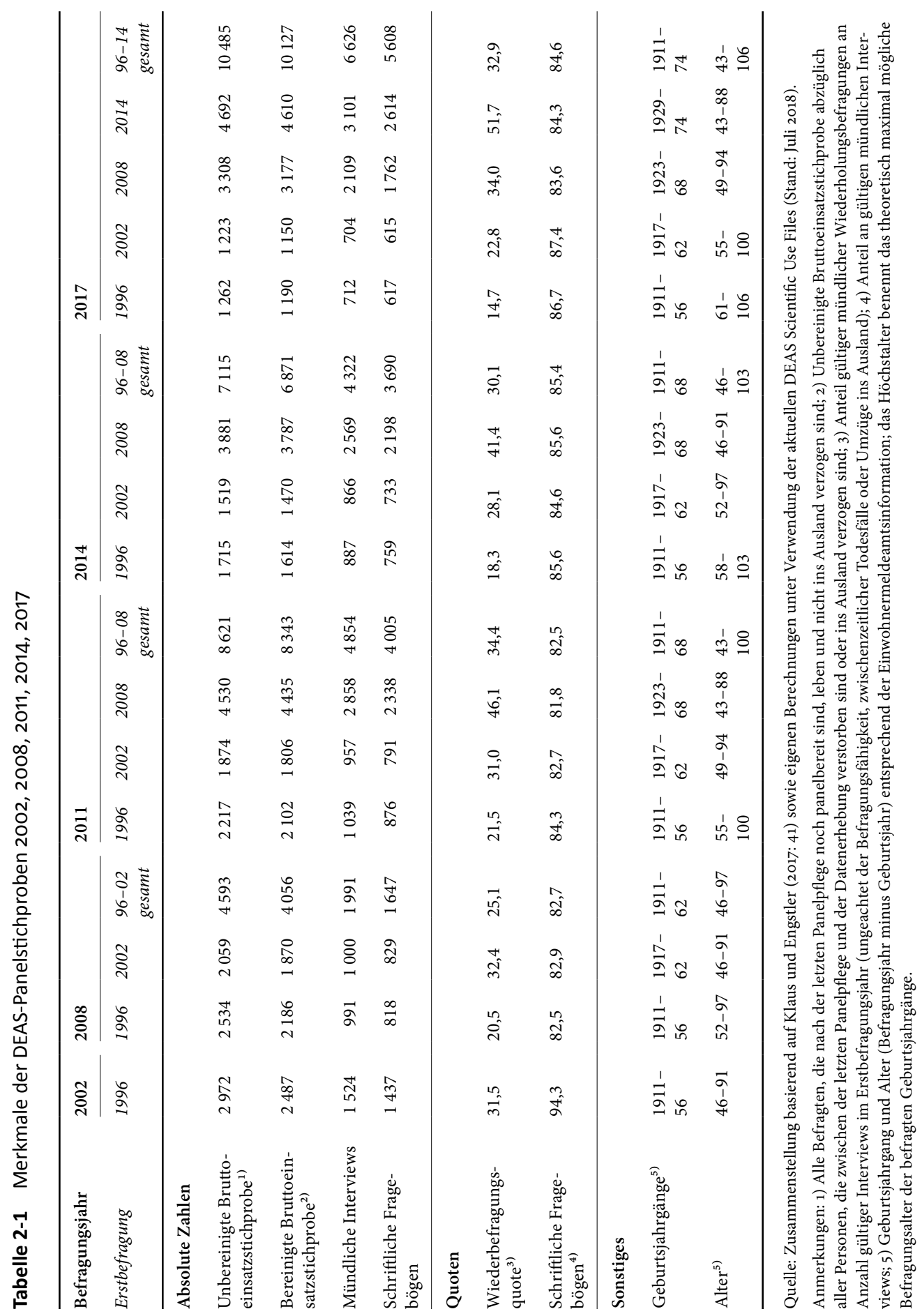


wicklung zeigt sich auch in mittelfristiger Perspektive: So haben von den im Jahr 1996 Erstbefragten zwölf Jahre später (2008) 21 Prozent teilgenommen, von den im Jahr 2002 Erstbefragten ebenfalls zwölf Jahre später (2014) 28 Prozent. Unterstützt wird dieser Trend durch eine gestiegene Lebenserwartung sowie eine zeitliche Ausdehnung der selbstständigen Lebensführung im eigenen Haushalt. Das sind Entwicklungen, die die Erreichbarkeit und Befragbarkeit der Erstteilnehmenden verbessern beziehungsweise verlängern. Hinzu kommt eine engmaschigere Panelpflege: Seit 2012 werden Studienteilnehmerinnen und -teilnehmer jährlich kontaktiert, um deren Adressen zu aktualisieren sowie deren Studienbindung durch das Versenden von Ergebnis- und Informationsbroschüren zu erhöhen. Der Anstieg der ersten Wiederholungsbefragungsquote der 2008 und 2014 Erstbefragten von 46 auf 52 Prozent könnte zudem Folge einer stärkeren Selektion unter den potenziellen Studienteilnehmerinnen und -teilnehmern bei der Erstteilnahme sein: Hinter der gesunkenen Teilnahmequote in der Erstbefragung könnte stehen, dass sich verstärkt Personen mit einer höheren Bleibemotivation zur Erstteilnahme entscheiden.

Erwartungsgemäß gestalten sich erneute Studienteilnahmen altersspezifisch (Abbildung 2-3). Grundsätzlich sind die Ausfälle in der ältesten Befragtengruppe am stärksten: So haben lediglich 19 Prozent der 70- bis 85-Jährigen der Erstbefragung 1996 sechs Jahre später erneut teilgenommen. Die vergleichbaren Quoten der beiden jüngeren Altersgruppen liegen bei 33 Prozent (55- bis 69-Jährige) und 39 Prozent (40- bis 54-Jährige). Dieser Alterseffekt geht auf zwei Mechanismen zurück: Erstens ist in der ältesten Befragtengruppe die reale Sterblichkeit am größten. Zweitens sinken in diesem Alter Befragbarkeit und Erreichbarkeit aufgrund abnehmender Gesundheit, zunehmender Pflegebedürftigkeit und damit verbundener
Krankenhaus- oder Heimaufenthalte (weiterführend zur Befragung alter Menschen: Motel-Klingebiel, Klaus, \& Simonson 2014).

Ein zweiter Alterseffekt deutet sich an: In den letzten Jahren bewegt sich die Wiederteilnahme der jüngsten Altersgruppe (40- bis 54-Jährige) leicht unter der der mittleren Altersgruppe (55- bis 69-Jährige). Dies zeigt sich für die erste Wiederholungsbefragung der Erstbefragten aus den Jahren 2008 und 2014. Diese Entwicklung könnte Folge einer steigenden räumlichen Mobilität sowie (zeitgleicher) beruflicher und familienbezogener Verpflichtungen sein, die vor allem im mittleren Erwachsenenalter zum Tragen kommen und wodurch Erreichbarkeit, Teilnahmemöglichkeiten und -motivation herabgesetzt werden.

Geschlechterunterschiede hinsichtlich des Verbleibens in der Studie fallen weniger deutlich aus (ohne Abbildung): Unabhängig vom Jahr der Erstteilnahme sind die Quoten der ersten Wiederholungsbefragung für Frauen (42 Prozent) und Männer (43 Prozent) annähernd gleich. Das ist zunächst in Anbetracht der geringeren Lebenserwartung der Männer überraschend. ${ }^{7}$ Möglicherweise wird die geringere Lebenserwartung der Männer dadurch ausgeglichen, dass sie anders als Frauen länger im privaten Haushalt leben anstatt in für Interviewerinnen und Interviewer schwer zugänglichen Institutionen wie Pflegeheimen. Zudem sind Frauen im Alter häufiger von gesundheitlichen Einschränkungen betroffen als Männer. Darüber hinaus wirken sich weitere Befragtenmerkmale auf die Wiederteilnahme aus, die offenbar geschlechtsspezifisch verteilt sind und auf diese Weise Geschlechterunterschiede verdecken (z.B. das im Vergleich zu älteren Frauen höhere Bildungsniveau der älteren Männer). Die nachfolgenden Selektivitätsanalysen belegen einen positiven Wiederbefragungseffekt des weiblichen Geschlechts.
7 Männer haben eine durchschnittliche Lebenserwartung von 78 Jahren im Vergleich zu 83 Jahren bei Frauen (auf Basis der Periodensterbetafel 2013/2015, vgl. www.gerostat.de). 
24 | Längsschnittliches Design, Inhalte und Methodik des Deutschen Alterssurveys

Abbildung 2-3 Wiederbefragungsquoten 1996-2017, getrennt nach Altersgruppen

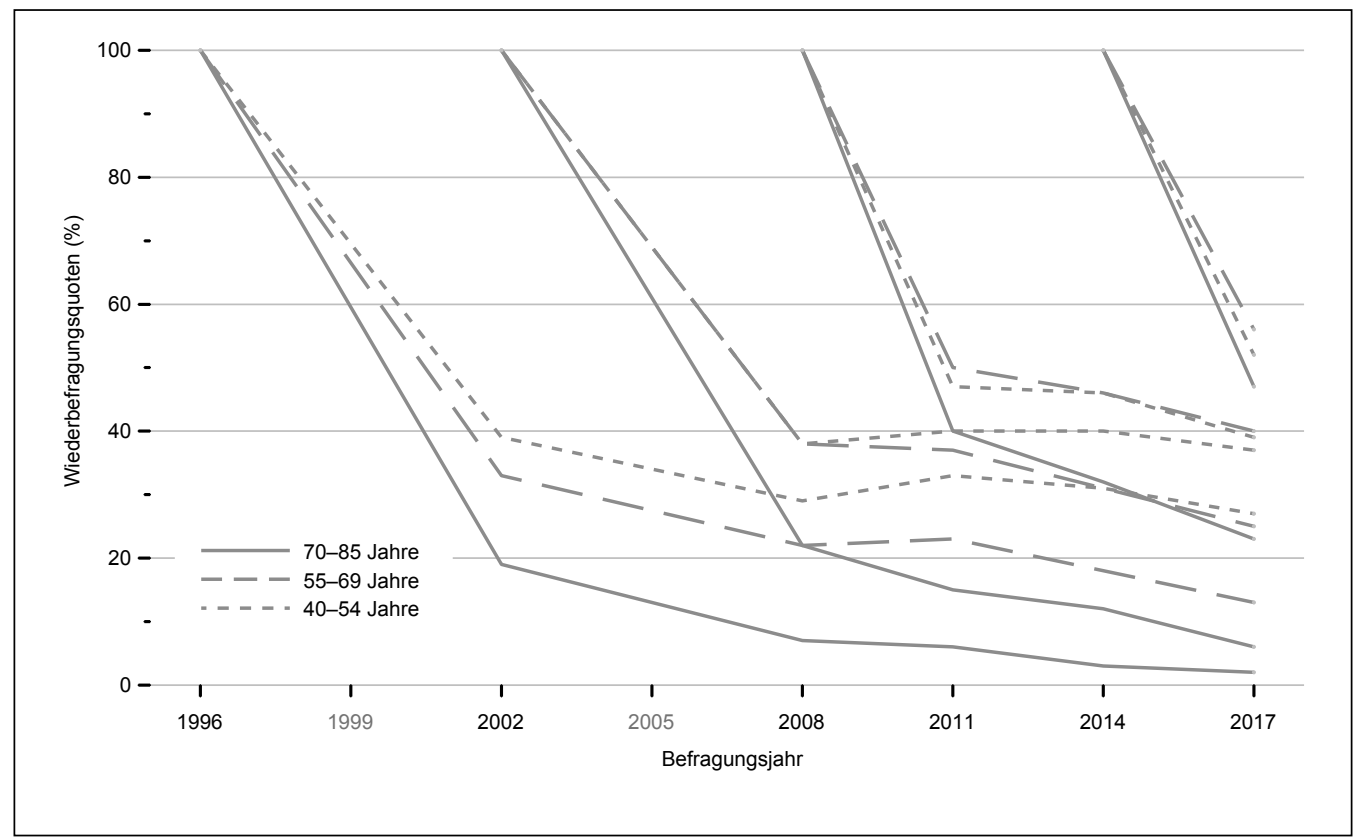

\begin{tabular}{|c|c|c|c|c|c|c|c|}
\hline \multirow[b]{2}{*}{ Erstbefragungsjahr } & \multirow[b]{2}{*}{ Altersgruppen } & \multicolumn{6}{|c|}{ Wiederbefragungsquoten (\%) } \\
\hline & & 1996 & 2002 & 2008 & 2011 & 2014 & 2017 \\
\hline \multirow[t]{3}{*}{ B1996 } & 40-54 Jahre & 100 & 39 & 29 & 33 & 31 & 27 \\
\hline & 55-69 Jahre & 100 & 33 & 22 & 23 & 18 & 13 \\
\hline & 70-85 Jahre & 100 & 19 & 7 & 6 & 3 & 2 \\
\hline \multirow[t]{3}{*}{ B2002 } & 40-54 Jahre & & 100 & 38 & 40 & 40 & 37 \\
\hline & 55-69 Jahre & & 100 & 38 & 37 & 31 & 25 \\
\hline & 70-85 Jahre & & 100 & 22 & 15 & 12 & 6 \\
\hline \multirow[t]{3}{*}{ B2008 } & 40-54 Jahre & & & 100 & 47 & 46 & 39 \\
\hline & 55-69 Jahre & & & 100 & 50 & 46 & 40 \\
\hline & 70-85 Jahre & & & 100 & 40 & 32 & 23 \\
\hline \multirow[t]{3}{*}{ B2014 } & 40-54 Jahre & & & & & 100 & 52 \\
\hline & 55-69 Jahre & & & & & 100 & 56 \\
\hline & $70-85$ Jahre & & & & & 100 & 47 \\
\hline
\end{tabular}

Quelle: DEAS 1996-2017. 


\section{Systematische Ausfälle in den Längsschnittstichproben}

Weiterführende Analysen unter Berücksichtigung mehrerer Merkmale der Befragungspersonen spezifizieren die eben beschriebenen alters- und geschlechtsspezifischen Ausfälle. Zudem ergänzen sie weitere - zumeist aus der Literatur bekannte - Effekte. In Abbildung 2-4 sind die untersuchten Einflussfaktoren in ihrer Vorhersagestärke für die Teilnahme an der aktuellen Befragung 2017 zusammengestellt. ${ }^{8}$ Berücksichtigte Einflussfaktoren sind Befragtenmerkmale, die aus deren Erstbefragung stammen. Ausgewählt wurden erstens sozio-demografische und -ökonomische Merkmale, deren Relevanz aus früheren DEAS-Wellen sowie der Literatur bekannt sind (Klaus \& Engstler 2017: 40): Alter (gruppiert), Geschlecht, Region (Ost- vs. Westdeutschland), städtisches Umfeld ab 20000 Einwohnerinnen und Einwohnern, Partnerschaft (ja/nein), Kinderzahl $(0-3+)$, subjektiv eingeschätzter Lebensstandard (1-5), Bildungsniveau (3-stufig nach der International Standard Classification of Education (ISCED)). Zweitens wurden zentrale Untersuchungsvariablen des vorliegenden Bandes geprüft: subjektive Gesundheit (1-5), Netzwerkgröße (1-6+), Enkelbetreuung (ja/ nein), mittlere Zufriedenheit mit Freizeit, Familienbeziehungen sowie Freundinnen oder Freunden und Bekannten (1-5), Ausübung eines Ehrenamtes (ja/nein). Ziel ist die Identifikation möglicher Panelselektivitäten, um sie in den Analysen entsprechend berücksichtigen zu können.

Die Ergebnisse bestätigen eine Reihe bekannter Mechanismen: Als erstes zeigt sich, dass nach Kontrolle der beschriebenen Merkmale, Frauen eine höhere Wiederbefragungswahrscheinlichkeit haben als Männer. Dieser Geschlechterunterschied wird erst nach Kontrolle des Bildungsniveaus sichtbar (eine schrittweise Aufnahme der Befragtenmerk- male ist hier nicht abgebildet). Das bedeutet: Offenbar wird der für Männer aufgrund ihrer geringeren Lebenserwartung erwartete frühere Ausfall aus der Studie dadurch kompensiert, dass ihre Bleibewahrscheinlichkeit angesichts ihrer durchschnittlich höheren Bildung erhöht ist.

Zweitens bestätigt sich der in Abbildung 2-4 illustrierte, nicht-lineare Alterseffekt: Während die Teilnahmewahrscheinlichkeit der 50-bis 59-Jährigen gegenüber den 40-bis 49-Jährigen höher ist, liegt sie in der ältesten Altersgruppe der über 70-Jährigen unter der Teilnahmewahrscheinlichkeit der jüngsten Erstbefragten. Einzelmodelle, getrennt nach dem Erstbefragungsjahr (ohne Abbildung), spezifizieren diesen Effekt: So zeigt sich die vergleichsweise geringere Wiederbefragungswahrscheinlichkeit der Befragten zwischen 40 und 49 Jahren vor allem in den ersten ein bis zwei Folgebefragungen - womöglich Ausdruck einer schlechten Erreichbarkeit und zeitlichen Verfügbarkeit in diesem mittleren Lebensalter. Hingegen zeigt sich der negative Effekt des hohen Alters auf die Teilnahmewahrscheinlichkeit vor allem langfristig, das heißt in vorangeschrittenen Panelwellen. Hier kommen die Folgen gesundheitlicher Einschränkungen und des zunehmenden Sterberisikos unter den alten und sehr alten Befragten zum Tragen. Weitere Effekte zeigen, dass Befragte in Städten ab 20000 Einwohnerinnen und Einwohnern sowie Befragte, die eine Partnerin oder einen Partner haben, mit höherer Wahrscheinlichkeit erneut teilnehmen. Ebenso erhöht sich die Wiederbefragungswahrscheinlichkeit mit dem individuellen Bildungsniveau sowie mit dem subjektiv wahrgenommenen Lebensstandard.

Auch an drei der zentralen Untersuchungsvariablen dieses Bandes sind Teilnahmeeffekte geknüpft: Gesunde, sozial gut eingebundene sowie ehrenamtlich aktive Befragungspersonen haben eine höhere Bleibewahrscheinlichkeit in der Stu-

8 Werte größer als eins verweisen auf eine höhere Wahrscheinlichkeit der Teilnahme 2017; Werte kleiner eins auf eine geringere Teilnahmewahrscheinlichkeit. 
Abbildung 2-4 Teilnahme an der Befragung 2017 (in Abhängigkeit von Merkmalen der Erstbefragung)

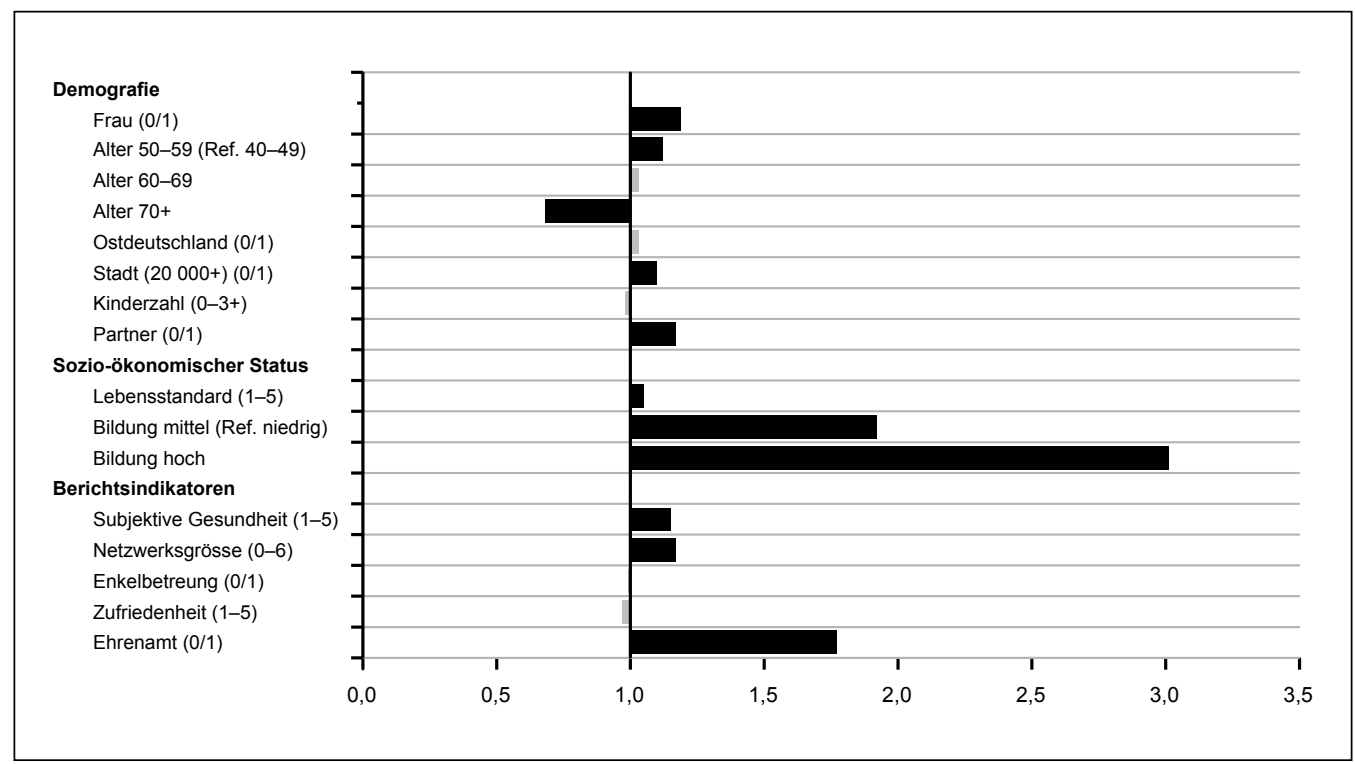

Daten: DEAS 1996-2017, $\mathrm{n}=18205$.

Logistische Regression: Odds Ratios; schwarze Balken: $\mathrm{p}<0,05$; Pseudo- $\mathrm{R}^{2}=0,07$.

die. Entsprechend sind sie in den Panelstichproben überrepräsentiert gegenüber Personen, die ihre Gesundheit schlecht einschätzen, wenig enge Netzwerkpersonen haben oder kein Ehrenamt bekleiden. Das bedeutet: Die auf der Grundlage des Deutschen Alterssurveys geschätzten Alternsverläufe können die Gesundheit, die soziale Einbindung sowie das ehrenamtliche Engagement leicht überschätzen.
Allerdings handelt es sich hierbei erstens um weitgehend $\mathrm{zu}$ erwartende und nicht vollständig in der Stichprobenumsetzung vermeidbare Selektionsprozesse. Zweitens verweisen die beinahe durchweg geringen Effektstärken - außer für Bildung und ehrenamtliches Engagement - auf Tendenzen anstatt auf starke Effekte.

\subsection{Erhebungsinhalte und -verfahren}

Der Deutsche Alterssurvey (DEAS) hat den Anspruch, umfassend Auskunft über die objektiven und subjektiven Lebensumstände der Menschen in der zweiten Lebenshälfte, deren Entwicklungen im Verlauf des Älterwerdens und im Zuge des gesellschaftlichen Wandels zu geben. Daher werden mit den Erhebungsinstrumenten zahlreiche Individu- almerkmale aus vielen Lebensbereichen wiederholt erhoben. Das Erhebungsprogramm zeichnet sich einerseits durch eine hohe Kontinuität der Inhalte aus, nimmt andererseits aber auch neue Themen oder temporäre Vertiefungen auf. Allgemeine Befragungsschwerpunkte sind: 
- Berufliche Entwicklung, Arbeit und Ruhestand

- Außerberufliche Tätigkeiten und ehrenamtliches Engagement

- Partnerschaft, Familie und Generationenbeziehungen

- Soziales Netzwerk und soziale Unterstützung

- Gesundheit und Gesundheitsverhalten

- Hilfe- und Pflegebedürftigkeit

- Haushalt, Wohnen und Mobilität

- Wirtschaftliche Lage, Altersvorsorge und finanzielle Unterstützung

- Lebensqualität und Wohlbefinden

- Einstellungen, Werte, Normen und Altersbilder

- Soziodemografische Grunddaten, Migrationshintergrund

Damit deckt der Deutsche Alterssurvey ein breites Themenspektrum ab und ermöglicht die Bearbeitung und Verknüpfung von Fragestellungen aus verschiedenen Disziplinen, insbesondere der Gerontologie, Psychologie, Soziologie, Ökonomie, Public Health und Sozialpolitikforschung.

Für die Datenerhebung werden in jeder Welle mehrere Erhebungsverfahren eingesetzt. Mit allen Befragten wird zunächst vor Ort ein persönliches mündliches Interview unter Einsatz eines standardisierten Fragebogens in deutscher Sprache durchgeführt. Seit 2008 erfolgt dieser umfängliche Teil der persönlichen Befragung computerunterstützt mit einem elektronischen Fragebogen (CAPI). Dies ermöglicht eine sehr differenzierte Filterführung der Fragenauswahl, -abfolge und -formulierung, für die seit 2011 bei Panelinterviews auch Merkmale aus dem letzten vorhergehenden Interview (sogenannte Preloads) der Befragungsperson einbezogen werden. Die durchschnittliche Dauer des mündlichen Interviews ist in den Folgeinterviews etwas kürzer als beim Einstiegsinterview. Im Jahr 2017, in dem nur Interviews mit in den Vorwellen bereits befragten Studienteilnehmenden des Deutschen Alterssurveys geführt wurden, betrug die durchschnittliche Befragungszeit $91 \mathrm{Mi}-$ nuten.

Mit zunehmendem Alter der DEAS-Teilnehmenden im Verlauf der Langzeitstudie steigt der
Anteil derjenigen, die aus gesundheitlichen Gründen Schwierigkeiten haben, an dem längeren Interview teilzunehmen. Zwar gibt es bereits seit der zweiten Erhebungswelle im Jahr 2002 die Möglichkeit, dass sich Befragte im Interview durch eine Person ihres Vertrauens unterstützen lassen. Dies diente aber in erster Linie zur sprachlichen Unterstützung von Personen, deren Muttersprache nicht Deutsch ist. Im Jahr 2017 wurde nun erstmals die Möglichkeit eingeführt, dass Befragungspersonen, die sich aus gesundheitlichen Gründen als stark eingeschränkt oder nicht mehr befragungsfähig sahen, eine Stellvertreterperson benennen können, die mit ihnen zusammen oder an ihrer Stelle als Proxy auf die Interviewfragen antwortet. Die Durchführung von Proxy-Interviews mit einem reduzierten Fragenkatalog (unter anderem ohne Fragen zu subjektiven Bewertungen) ist dabei nur möglich, wenn die Zielperson dies bei der Kontaktaufnahme wünscht, ihr Einverständnis gibt und der Interviewerin oder dem Interviewer die stellvertretende Person namentlich benennt. In der Erhebungswelle des Jahres 2017 kam es unter diesen Voraussetzungen zu 22 gültigen Stellvertreterinterviews.

Im Verlauf des mündlichen Interviews und direkt danach werden zusätzlich verschiedene Tests durchgeführt (nicht bei Proxy-Interviews). Seit 2002 wird ein Zahlen-Zeichen-Test zur Messung der Verarbeitungsgeschwindigkeit als Teilaspekt der kognitiven Leistungsfähigkeit eingesetzt (Hoyer, Stawski, Wasylyshyn, \& Verhaeghen 2004; Tewes 1994) und seit 2008 ein Lungenfunktionstest als objektiver Indikator für die körperliche Gesundheit (Fragoso, Gahbauer, van Ness, Concato, \& Gill 2008). Im Jahr 2017 haben 92 Prozent der Befragten am Lungenfunktionstest und 85 Prozent am Zahlen-Zeichen-Test teilgenommen.

Nach dem mündlichen Interview werden die Befragten gebeten, einen schriftlichen Fragebogen auszufüllen (Drop-Off). Hier werden vor allem Einstellungen und subjektive Einschätzungen erhoben, sensible Fragen wie zum Einkommen und der Gesundheit gestellt und Merkmale erfragt, die keine differenzierte Fragenführung benötigen. 
Auch verschiedene psychologische Skalen werden im Drop-Off erhoben. Die Beantwortung erfolgt überwiegend in Form des Ankreuzens aus vorgegebenen Antwortmöglichkeiten. Bis zum Jahr 2014 gab es den Drop-Off ausschließlich als Papierfragebogen, der von den Befragten mit einem Stift ausgefüllt werden muss (Paper Pencil). Im Jahr 2017 wurde erstmals die zusätzliche Option eingeführt, den Drop-Off-Fragebogen in elektronischer Form über einen Internetzugang auszufüllen (CAWI: computer assisted web interview). Rund 18 Prozent der Ausfüllenden entschieden sich für die elektronische Variante. Je jünger die Befragten waren, desto häufiger wählten sie die Online-Version. Insgesamt liegen für das Jahr 2017 von 5608 der 6626 Interviewten gültige Drop-Off-Informationen vor. Damit haben rund 85 Prozent der Panelbefragten des Jahres 2017 nach dem mündlichen Interview auch den Selbstausfüller-Fragebogen beantwortet. Die über die Jahre leicht sinkende Ausfüllquote konnte durch die Option der Online-Variante stabilisiert werden.

Bei Zielpersonen, die zwar erreicht, aber aus unterschiedlichen Gründen nicht interviewt werden können und für die auch kein Stellvertreterinterview durchgeführt werden kann, wird seit 2008 nach Möglichkeit ein Kurzfragebogen eingesetzt, den entweder die Zielperson selbst beantwortet oder eine Kontaktperson. Ziel ist die Erfassung einiger Basisinformationen wie Wohnform, Gesundheitszustand, Staatsangehörigkeit, Bildung oder Lebenszufriedenheit, um auf diese Weise die
Selektivität der (temporären) Ausfälle besser beschreiben zu können. Im Jahr 2017 konnte für 354 Personen der Panelstichprobe ein Kurzfragebogen eingesetzt werden. Das entspricht rund 14 Prozent der nicht am Hauptinterview teilnehmenden Zielpersonen der bereinigten Bruttoeinsatzstichprobe (Schiel, Ruiz Marcos, Dickmann, Aust, \& Middendorf 2018: 33). In drei von vier Fällen wurde das Kurzinterview direkt mit der Zielperson geführt. Die geringe Bereitschaft zur Teilnahme an einem Kurzinterview ist auch aus anderen Altersstudien bekannt (Börsch-Supan, Brandt, Hunkler, Kneip, Korbmacher, Malter, Schaan, Stuck, \& Zuber 2013).

Ergänzend zu den Befragungsdaten bietet der Deutsche Alterssurvey weitere Daten, die das Wohnumfeld der Befragungspersonen auf unterschiedlichen räumlichen Ebenen beschreiben. Für alle Erhebungsjahre liegen Einschätzungen der Interviewenden zur Wohnung und zum Wohnumfeld der Befragten vor. Für die Befragten der Jahre 2002, 2008 und 2014 stehen quartiersbezogene Daten zur sozialstrukturellen Zusammensetzung der Wohngegend, zu Sozialmilieus, Kaufkraft oder zur Arbeitslosigkeit zur Verfügung. ${ }^{9}$ Auf Kreisebene können für alle Befragten und alle Erhebungsjahre ausgewählte Indikatoren und Karten zur Raum- und Stadtentwicklung (INKAR-Daten) vom Bundesinstitut für Bau-, Stadt- und Raumforschung genutzt werden wie beispielsweise zu Arbeitsmarkt, Bevölkerung, medizinischer Versorgung, Verkehr oder Wirtschaft.
9 Die Strukturdaten zum Wohnumfeld der Befragten (im Jahr 2002 nur für panelbereite Befragte) wurden von der Firma microm Micromarketing-Systeme und Consult $\mathrm{GmbH}$, Neuss, zur Verfügung gestellt.
Für die Befragung des Jahres 2014 liegen auch Wohnumfeldmerkmale der Firma infas $360^{\circ} \mathrm{GmbH}$, Bonn, vor. Geplant ist, diese auch für die DEAS-Welle des Jahres 2017 bereitzustellen. 


\subsection{Untersuchung der Alternsverläufe von Frauen und Männern}

Wie im Einleitungskapitel (Kapitel 1 Älterwerden von Frauen und Männern) dieses Bandes begründet, stehen die Darstellung und Beschreibung geschlechtsspezifischer Alternsverläufe hier im Mittelpunkt. Das Erkenntnissinteresse liegt auf folgenden vier Aspekten: Erstens wird die Richtung von durchschnittlichen Veränderungen beschrieben, die mit dem Älterwerden eintreten können. Zweitens geht es darum, mögliche Wendepunkte im Verlauf der zweiten Lebenshälfte zu identifizieren: Ab wann entwickeln sich Lebensbereiche und -situationen in eine andere Richtung als zuvor? Drittens gilt es aus dem Vergleich der Alternsverläufe von Frauen und Männern zu lernen. Gleichen sich die Alternsverläufe von Frauen und Männern oder nicht? Erleben sie gleiche oder unterschiedliche Veränderungen mit dem Älterwerden? Starten mögliche Veränderungen vom selben Ausgangsniveau oder nicht und in welchem Alter entwickeln sich die Verläufe der Geschlechter auseinander und gleichen sich gegebenenfalls wieder an? Schließlich wird viertens mit einem Vergleich aufeinander folgender Geburtskohorten geprüft, ob sich Unterschiede der Alternsverläufe von Frauen und Männern in der Kohortenfolge angleichen, vergrößern oder ob sie stabil bleiben.

Das Älterwerden von Frauen und Männern wird in diesem Band anhand der Alternsverläufe vom 40. bis zum 90. Lebensjahr untersucht. Das heißt, es wird eine lange Spanne von 50 Lebensjahren betrachtet. Grundlage hierfür ist eine Kombination der Daten der Geburtsjahrgänge von 1911 bis 1974, die bei ihrer Erstbefragung zwischen 40 und 85 Jahren alt waren und die über einen maximalen Zeitraum von 21 Jahren beobachtet und bis zu sechsmal befragt wurden. Eine solche modellbasierte Schätzung der Alternsverläufe über 50 Jahre hinweg wird möglich, indem die Daten individuell beobachteter Veränderungen im Alternsprozess von jüngeren, später geborenen und von älteren, früher geborenen Befragten teilweise überlappend aneinandergereiht werden. Zugleich liegen für viele Altersjahre jeweils Daten aus mehreren Geburtsjahrgängen vor. Beispielsweise liegen für die Geburtsjahrgänge 1931, 1937, 1943, 1946, 1949 und 1952 Angaben für das Alter von 65 Jahren vor aus unterschiedlichen Erhebungsjahren (1996, 2002, 2008, 2011, 2014, 2017).

Die Darstellung der Datenstruktur des Deutschen Alterssurveys in Abbildung 2-5 veranschaulicht dies am Beispiel ausgewählter Geburtsjahrgänge. Auf der y-Achse sind die Geburtsjahrgänge dargestellt und auf der $\mathrm{x}$-Achse das Lebensalter. Jede Zeile steht für den Lebensverlauf des jeweiligen Geburtsjahrgangs über die Altersspanne von 40 bis 90 Jahren. Für jeden Geburtsjahrgang ist jeweils die Altersspanne grau unterlegt, für die Befragungsdaten vorliegen - also maximal 21 Jahre. Die Darstellung illustriert nun beispielsweise für Personen, die im Jahr 1930 geboren sind, dass diese zum ersten Mal im Lebensalter von 66 Jahren befragt wurden und zwar im Rahmen der ersten Erhebungswelle im Jahr 1996. Ein Teil dieser Personen wurden später im Alter von $72,78,81,84$ und 87 Jahren wiederholt befragt (Erhebungen in den Jahren 2002, 2008, 2011, 2014 und 2017). Entsprechend liegen von ihnen Angaben vor, die die Altersspanne von 66 bis 87 abdecken. Um Verläufe über eine längere Altersspanne untersuchen zu können, die auch frühere Altersphasen einschließt, werden die Daten der früher geborenen Jahrgänge mit jenen der später geborenen Jahrgänge verknüpft. So liegen etwa für den Geburtsjahrgang 1956 Daten für die Alterspanne von 40 bis 61 Jahren vor.

Damit wird deutlich, dass die hier analysierten Alternsverläufe auf Schätzungen für Altersspannen basieren, für die nicht für alle Geburtsjahrgänge beobachtete Werte vorliegen. Das bedeutet, dass die Alternsverläufe insbesondere für jüngere Geburtskohorten bis zu einem Lebensalter geschätzt werden, das sie noch nicht erreicht haben. 
Somit geht in die Schätzung der allgemeinen Alternsverläufe die Schätzung zukünftiger Verläufe später geborener Jahrgänge als Fortschreibung beobachteter vergangener Verläufe früher geborener Geburtskohorten ein - unter der Annahme, dass sich der soziale Wandel graduell über nachfolgende Kohorten fortsetzt. In gleicher Weise wird für früher geborene Jahrgänge der Alternsverlauf in jüngeren Lebensjahren geschätzt, obwohl für diese in diesen jüngeren Altersphasen keine Befragungsdaten vorliegen. Eine Betrachtung der Alternsverläufe aufgrund der reichen Datenlage kann bis ins hohe Alter erfolgen. Begründet durch zu geringe Fallzahlen ist allerdings eine Darstellung jenseits des 90 . Lebensjahres nicht mehr verlässlich möglich.

Eine geschlechtsspezifische Schätzung von Alternsverläufen kann angesichts der überproportionalen Ziehung von Männern in den Basisstichproben sowie einer weitgehend abhängigen Wiederbefragungswahrscheinlichkeit realisiert werden. Darüber hinaus ist ein Vergleich von Frauen und Männern unterschiedlicher Geburtskohorten möglich. Dieser beschränkt sich auf folgende drei Geburtskohorten: 1930 bis 1939, 1940 bis 1949 und 1950 bis 1959. Wie Tabelle 2-2 zeigt, liegen für diese Geburtskohorten Werte aus dem gesamten DEAS-Beobachtungsfenster von 21 Jahren vor und innerhalb dieses Beobachtungsintervalls liegen für diese Kohorten ausreichend viele Datenpunkte vor, um Verläufe auf Grundlage statistischer Analysemodelle schätzen und vergleichen zu können. Darüber hinaus sind für diese ausgewählten Geburtskohorten die Fallzahlen ausreichend hoch, während später geborene (ab 1960 Geborene) und auch früher geborene (vor 1930 Geborene) Randkohorten weniger stark besetzt sind. Die kohortenspezifischen Alternsverläufe sowie die Geschlechterunterschiede in den kohortenspezifischen Alternsverläufen werden deshalb ausschließlich auf Basis der Daten der Geburtsjahrgänge 1930 bis 1959 geschätzt. Die kohortenspezifischen Alternsverläufe werden in den empirischen Kapiteln jeweils nur bis zu dem Lebensalter dargestellt, welches die Kohorten im aktuellen Erhebungsjahr 2017 erreicht haben, und bei den früher geborenen Kohorten erst ab dem Lebensalter, welches sie bei der Erstbefragung bereits erreicht hatten.

Tabelle 2-2 Fallzahlen nach Geburtskohorten

\begin{tabular}{lcccc}
\hline Geburtsjahrgänge & Anzahl der Befragten & Prozent & $\begin{array}{l}\text { Anzahl der Beob- } \\
\text { achtungen }\end{array}$ & $\begin{array}{l}\text { Theoretisch maximale } \\
\text { Altersspanne }\end{array}$ \\
\hline $1911-1919$ & 523 & 2,6 & 615 & $77-106$ \\
$1920-1929$ & 2453 & 12,2 & 3736 & $67-97$ \\
$1930-1939$ & 4577 & 22,7 & 8845 & $57-87$ \\
$1940-1949$ & 4774 & 23,7 & 10335 & $40-77$ \\
$1950-1959$ & 4654 & 23,1 & 10072 & $40-67$ \\
$1960-1969$ & 2721 & 13,5 & 5208 & $40-57$ \\
$1970-1974$ & 427 & 2,1 & 635 & $40-47$ \\
Gesamt & 20129 & 100,0 & 39446 & $40-106$ \\
\hline
\end{tabular}




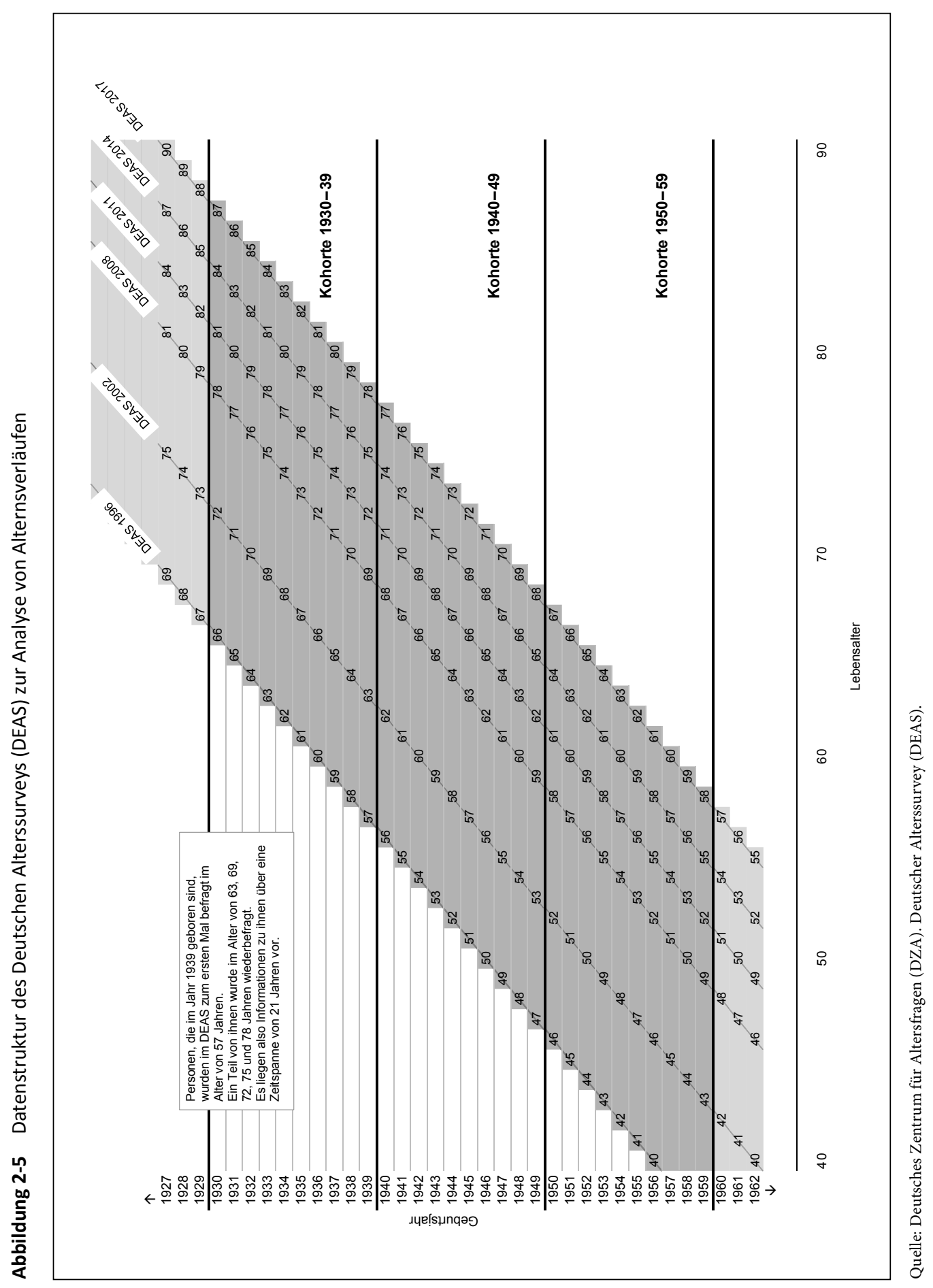


Die in den empirischen Kapiteln dieses Bandes dargestellten individuellen Alternsverläufe beschreiben durchschnittliche Verläufe. Daran kann abgelesen werden, welche Veränderungen eintreten, wenn Menschen älter werden, und zwar im Durchschnitt über alle Personen hinweg. Das Lebensalter ist in den Abbildungen jeweils auf der $\mathrm{x}$-Achse abgetragen. Dargestellt ist in den Alternsverläufen etwa, wie sich die Gesundheit mit dem Älterwerden im Durchschnitt verändert. Je nach Art des betrachteten Merkmals sind die in den Abbildungen berichteten Werte unterschiedlich $\mathrm{zu}$ interpretieren. Die Werte stehen entweder für den mittleren Wert zu einem spezifischen Alter im Alternsverlauf (etwa der mittleren subjektiven Gesundheit oder der mittleren Lebenszufriedenheit). Oder anhand von Alternsverläufen kann die Veränderung der mittleren Wahrscheinlichkeiten abgelesen werden, sich in einem bestimmten Zustand zu befinden (etwa unter klinisch auffälligen depressiven Symptomen zu leiden) oder eine bestimmte Aufgabe oder Tätigkeit auszuüben (etwa die Betreuung von Enkelkindern oder die Bekleidung eines Ehrenamtes). Obwohl hinter den dargestellten Ergebnissen unterschiedliche statistische Analyseverfahren stehen, können die Ergebnisse und Abbildungen über alle Kapitel hinweg einheitlich gelesen werden.

Um die Alternsverläufe über eine Altersspanne von 50 Jahren darstellen zu können, werden modellbasierte Schätzungen auf Basis der DEAS-Paneldaten 1996 bis 2017 berechnet, unter Einbeziehung der Geburtsjahrgänge 1911 bis 1974. Um der Schichtung der Basisstichproben (nach Altersgruppe, Geschlecht und Ost- vs. Westdeutschland) Rechnung zu tragen, werden diese drei Merkmale grundsätzlich in alle Berechnungen einbezogen beziehungsweise kontrolliert. Alter geht zur Schätzung der Alternsverläufe per se als Zeitvariable in alle Modelle ein. Um Verzerrungen der Panelstichproben auszugleichen, da Personen mit bestimmten Merkmalen eine höhere Wahrscheinlichkeit haben an Wiederholungsbefragungen teilzunehmen als andere, werden durchweg nicht nur die Angaben von Mehrfachbefragten sondern auch von einmalig Befragten einbezogen. Auf diese Weise kann einer verzerrten Schätzung von Alternsverläufen entgegen gewirkt werden wie etwa einer Unterschätzung des Rückgangs der Gesundheit im Alternsverlauf.

Um in der Schätzung zu berücksichtigen, dass ein Merkmal im Alternsverlauf nicht nur linear ansteigt oder absinkt, sondern beispielsweise auch beschleunigt ansteigen oder absinken kann oder sich auch in verschiedene Richtungen entwickeln kann, wird der Altersindikator zusätzlich in quadrierter Form in die Modellschätzung aufgenommen und nicht nur in linearer Form. Die Schätzungen zeigen dann, ob der entsprechende durchschnittliche Verlauf besser als linear oder als quadratisch beschrieben werden kann. Geschlecht wird in die Schätzungen aufgenommen, um zu prüfen, ob sich die Alternsverläufe für Frauen und Männer unterschiedlich darstellen und/oder ob sie sich in bestimmten Lebensaltern unterscheiden. Schließlich werden vergleichende Schätzungen für die drei Geburtskohorten 1930 bis 1939, 1940 bis 1949 und 1950 bis 1959 berechnet, um der Frage nachzugehen, ob sich die (möglicherweise geschlechtsspezifischen) Alternsverläufe der später geborenen Kohorten statistisch signifikant von den früher geborenen Kohorten unterscheiden sowie ob sich Frauen und Männer später geborener Kohorten in ähnlicher Weise in ihren Alternsverläufen unterscheiden (oder nicht) wie die Frauen und Männer früher geborener Kohorten. Aufgrund der Überschneidungen der Altersspannen, für die pro Kohorte Daten vorliegen, ist es möglich, die Kohorten zu vergleichen (in den Modellen werden lineare Kohorteneffekte angenommen).

Grundsätzlich werden alle Verläufe und Unterschiede auf statistische Signifikanz getestet. Dazu wird bei den statistischen Tests das Signifikanzniveau - dem etablierten Standard entsprechend - auf fünf Prozent $(\mathrm{p}<0,05)$ gesetzt. Je nach Datenlage kommen in den einzelnen Kapiteln unterschiedliche Analyseverfahren zur Anwendung, die jedoch alle derselben Grundkonzeption einer längsschnittlichen Schätzung von (geschlechtsspezifischen) Alternseffekten folgen: Dual Change 
Score Modelle, längsschnittliche Mehrebenenregressionsmodelle, logistische Random-Effects-Panelregressionen. Die Berechnungen wurden unter
Verwendung verschiedener Statistik-Programme realisiert (Mplus, SPSS, Stata, R).

\subsection{Ausblick}

Aufgrund der Langzeitbeobachtung von Personen in ihrer zweiten Lebenshälfte über einen Zeitraum von 21 Jahren und über bis zu sechs Befragungsteilnahmen ist mit dem Deutschen Alterssurvey (DEAS) eine umfangreiche Datengrundlage entstanden. Sie ermöglicht Analysen, die für Deutschland bislang kaum in vergleichbarer Form vorliegen: Die Untersuchung geschlechtsspezifischer Alternsverläufe zwischen dem 40. und 90. Lebensjahr und ihrer Dynamik in der historischen Abfolge der Geburtskohorten zwischen 1930 und 1959. Auf Basis dieser Daten kann wichtigen Fragen nachgegangen werden: Gibt es Anzeichen dafür, dass sich die Veränderungen, die mit dem Älterwerden einhergehen, bei Frauen und Männern unterscheiden? Und unterliegen die geschlechtsspezifischen Prozesse des Älterwerdens sozialem Wandel? Antworten auf diese Fragen werden in den folgenden Kapiteln gegeben. Es werden für die zentralen Lebensbereiche Gesundheit, Lebenszufriedenheit und depressive Symptome, soziale Isolation und Einsamkeit, Sorgetätigkeiten und Ehrenamt Geschlechterunterschiede in den Alternsverläufen dargestellt. Zudem werden geschlechtsspezifische Alternsverläufe aufeinanderfolgender Geburtskohorten untersucht, um festzustellen, ob und in welchen Bereichen eine Angleichung oder auch Ausdifferenzierung des Älterwerdens zwischen Frauen und Männern stattgefunden hat und in welchen (noch) nicht.

\section{Literatur}

Börsch-Supan, A., Brandt, M., Hunkler, C., Kneip, T., Korbmacher, J., Malter, F., Schaan, B., Stuck, S., \& Zuber, S. (2013). Data resource profile: the Survey of Health, Ageing and Retirement in Europe (SHARE). International Journal of Epidemiology, 42(4), 992-1001. doi: 10.1093/ije/dyt088.

Fragoso, C. A., Gahbauer, E. A., Ness, P. H. van, Concato, J., \& Gill, T. M. (2008). Peak expiratory flow as a predictor of subsequent disability and death in community living older persons. Journal of the American Geriatrics Society, 56(6), 1014-1020. doi: 10.1111/j.1532-5415.2008.01687.x.

Hoyer, W. J., Stawski, R. S., Wasylyshyn, C., \& Verhaeghen, P. (2004). Adult age and digit symbol substitution performance: a meta-analysis. Psychology and Aging, 19(1), 211-214. doi: 10.1037/08827974.19.1.211.

Klaus, D., \& Engstler, H. (2017). Daten und Methoden des Deutschen Alterssurveys. In: K. Mahne, J. K. Wolff, J. Simonson \& C. Tesch-Römer (Hrsg.) Altern im Wandel. Zwei Jahrzehnte Deutscher Alterssurvey (S. 29-45). Wiesbaden: Springer VS.

Mahne, K., Wolff, J. K., Simonson, J., \& Tesch-Römer, C. (Hrsg.). (2017). Altern im Wandel. Zwei Jahrzehnte Deutscher Alterssurvey (DEAS). Wiesbaden: Springer VS.

Motel-Klingebiel, A., Klaus, D., \& Simonson, J. (2014). Befragungen von älteren und alten Menschen. In: N. Baur \& J. Blasius (Hrsg.) Handbuch Methoden der empirischen Sozialforschung (S. 781-786). Wiesbaden: Springer VS. 
Motel-Klingebiel, A., Wurm, S., \& Tesch-Römer, C. (Hrsg.). (2010). Altern im Wandel. Befunde des Deutschen Alterssurveys (DEAS). Stuttgart: Kohlhammer.

Schiel, S., Ruiz Marcos, J., Dickmann, C., Aust, F., \& Middendorf, L. (2018). Deutscher Alterssurvey (DEAS): Durchführung der 6. Erhebungswelle 2017. Bonn: infas.

Tesch-Römer, C., Engstler, H., \& Wurm, S. (Hrsg.). (2006). Altwerden in Deutschland. Sozialer Wandel und individuelle Entwicklung in der zweiten Lebenshälfte. Wiesbaden: VS Verlag für Sozialwissenschaften.

Tewes, U. (1994). HAWIE-R: Hamburg-Wechsler-Intelligenztest für Erwachsene, Revision 1991. Handbuch und Testanweisung. Bern: Huber.

Open Access Dieses Kapitel wird unter der Creative Commons Namensnennung 4.0 International Lizenz (http:// creativecommons.org/licenses/by/4.0/deed.de) veröffentlicht, welche die Nutzung, Vervielfältigung, Bearbeitung, Verbreitung und Wiedergabe in jeglichem Medium und Format erlaubt, sofern Sie den/die ursprünglichen Autor(en) und die Quelle ordnungsgemäß nennen, einen Link zur Creative Commons Lizenz beifügen und angeben, ob Änderungen vorgenommen wurden.

Die in diesem Kapitel enthaltenen Bilder und sonstiges Drittmaterial unterliegen ebenfalls der genannten Creative Commons Lizenz, sofern sich aus der Abbildungslegende nichts anderes ergibt. Sofern das betreffende Material nicht unter der genannten Creative Commons Lizenz steht und die betreffende Handlung nicht nach gesetzlichen Vorschriften erlaubt ist, ist für die oben aufgeführten Weiterverwendungen des Materials die Einwilligung des jeweiligen Rechteinhabers einzuholen. 


\title{
3. Funktionale und subjektive Gesundheit bei Frauen und Männern im Verlauf der zweiten Lebenshälfte
}

\author{
Svenja M. Spuling, Anja Cengia und Markus Wettstein
}

\section{Kernaussagen}

Mit steigendem Alter berichten Frauen und Männer eine zunehmend eingeschränkte funktionale Gesundheit, und sie bewerten ihre Gesundheit weniger positiv: Die funktionale Gesundheit, das heißt die selbstständige Mobilität und die allgemeine Alltagskompetenz, nimmt mit zunehmendem Alter ab. Auch die individuelle subjektive Bewertung der eigenen Gesundheit verschlechtert sich mit zunehmendem Alter, sie nimmt aber über den Alternsverlauf von 40 bis 90 Jahren insgesamt weniger stark ab als die funktionale Gesundheit.

Frauen berichten zwar eine stärker eingeschränkte funktionale Gesundheit als Männer - und das im Alternsverlauf mit zunehmenden Maße -, aber subjektiv bewerten Frauen und Männer ihre Gesundheit ähnlich über die gesamte zweite Lebenshälfte: Frauen haben bereits im Alter von 40 Jahren eine schlechtere funktionale Gesundheit als Männer, und dieser Geschlechterunterschied nimmt mit steigendem Alter zu. Dagegen unterscheiden sich Frauen und Männer weder im Alter von 40 Jahren noch im Alternsverlauf hinsichtlich ihrer subjektiven Gesundheit. Frauen und Männer bewerten ihre Gesundheit demnach subjektiv ähnlich, obwohl sie sich in ihrer funktionalen Gesundheit unterscheiden.

Später Geborene kommen voraussichtlich mit einer besseren funktionalen Gesundheit ins höhere Alter als früher Geborene: In der ersten Hälfte des fünften Lebensjahrzehnts haben früher geborene Kohorten eine durchschnittlich bessere funktionale Gesundheit als später geborene Kohorten. Dies gilt für Frauen und Männer gleichermaßen. Im höheren Alter werden jedoch Frauen und Männer später geborener Kohorten voraussichtlich eine bessere funktionale Gesundheit und eine geringere Abnahme der funktionalen Gesundheit aufweisen als die Frauen und Männer früher geborener Kohorten. Dagegen unterscheidet sich bei der subjektiven Gesundheit weder das mittlere Ausgangsniveau mit Anfang 40 noch der Alternsverlauf zwischen den untersuchten Geburtskohorten, dies gilt für Frauen und Männer. 


\subsection{Einleitung}

Gesundheit hat für alle Menschen einen hohen Stellenwert, doch oft wird ihr erst in der zweiten Lebenshälfte Aufmerksamkeit zuteil (Böhm, TeschRömer, \& Ziese 2009). Der eigene Gesundheitszustand beeinflusst zum einen maßgeblich viele persönliche Lebensbereiche: Gesunde Menschen leben meist selbstständiger als Menschen, die gesundheitlich eingeschränkt sind. Für Gesunde ist das Risiko, hilfs- und pflegebedürftig zu werden, geringer, ihr allgemeines Wohlbefinden ist höher und sie können ihre Freizeit autonom gestalten und soziale Kontakte pflegen (Schöllgen, Morack, Infurna, Ram, \& Gerstorf 2016). Zum anderen hat die Gesundheit eine hohe soziale Relevanz, weil sie sich auch darauf auswirkt, ob Menschen an der Gesellschaft teilhaben, sich freiwillig engagieren oder für andere sorgen können.

Lange Zeit wurde Gesundheit mit der Abwesenheit körperlicher Erkrankungen gleichgesetzt. Zur Definition dessen, was unter Gesundheit zu verstehen ist, wurden ausschließlich medizinische Gesundheitsmodelle herangezogen, denen eine eindimensionale Vorstellung von "gesund“ versus „krank“ zugrunde liegt. Um den Gesundheitszustand von Menschen in seiner ganzen Breite zu erfassen, ist jedoch ein mehrdimensionales Konzept von Gesundheit erforderlich, das neben der körperlichen Gesundheit etwa auch die körperliche Funktionsfähigkeit, die subjektive Gesundheitsbewertung sowie die psychische Gesundheit berücksichtigt (vgl. World Health Organization 2002). Im vorliegenden Kapitel werden die beiden Dimensionen der funktionalen Gesundheit und der subjektiven Gesundheit eingehender beleuchtet, Informationen zur psychischen Gesundheit bietet das Kapitel 4 Lebenszufriedenheit und depressive Symptome in diesem Band.

Gesundheitszustände können sich zum einen über den Lebensverlauf verändern und zum anderen zwischen den Geschlechtern unterscheiden. Zudem differieren womöglich die Veränderungen in der Gesundheit von Frauen und Männern mit zunehmendem Alter. Um individuelle gesund- heitliche Veränderungen mit zunehmendem Alter umfassend abbilden zu können, ist ein langer Beobachtungszeitraum mit wiederholten Befragungen wichtig.

\section{Funktionale und subjektive Gesundheit im Alter}

Beim Konzept der funktionalen Gesundheit wird die körperliche Funktionsfähigkeit im Alltag betrachtet. Menschen sind funktional gesund, wenn sie in ihrer Alltagsmobilität, in ihrer selbstständigen Lebensführung beziehungsweise der allgemeinen Alltagskompetenz nicht eingeschränkt sind. Einschränkungen der funktionalen Gesundheit liegen vor, wenn Menschen Alltagsaufgaben, wie zum Beispiel Einkaufengehen, Treppensteigen, Putzen, Waschen oder Nahrungszubereitung und -aufnahme, nicht ohne fremde Hilfe bewältigen können (Baltes, Maas, Wilms, \& Borchelt 2010). Die körperliche Gesundheit (im Sinne der Anzahl an chronischen Erkrankungen) ist dabei auch ein Einflussfaktor - je schlechter die körperliche Gesundheit ist, desto wahrscheinlicher ist es, dass jemand im Alltag funktional eingeschränkt ist. Dabei bleibt aber zu beachten, dass allein das Vorhandensein einer oder mehrerer Erkrankungen nicht unbedingt zu einer funktionalen Einschränkung führen muss - viele Personen meistern auch mit mehreren Erkrankungen ihren Alltag ohne jegliche Einschränkungen.

Mit zunehmendem Alter berichten deutlich mehr Menschen Einschränkungen bei alltäglichen Aktivitäten (Wolff, Nowossadeck, \& Spuling 2017). Eine mögliche Erklärung hierfür ist das häufigere Auftreten von Multimorbidität - also das Vorliegen mehrerer Erkrankungen gleichzeitig - mit zunehmendem Alter, welche das Risiko für das Auftreten von funktionalen Einschränkungen erhöht. Durch altersphysiologische Prozesse, also biologische Veränderungen des alternden Körpers, erhöht sich mit zunehmendem Alter die Wahrscheinlich- 
keit, an chronischen Erkrankungen zu leiden sowie von Multimorbidität betroffen zu sein (Barnett, Mercer, Norbury, Watt, Wyke, \& Guthrie 2012). Diese altersphysiologischen Prozesse umfassen typischerweise unter anderem die Abnahme von Knochendichte, Muskelkraft, Arterienelastizität und Herzschlagvolumen. Zusätzliche Risikofaktoren für Erkrankungen sind Übergewicht sowie Lebensstilfaktoren, etwa Rauchen. Die Kombination aus diesen Risikofaktoren und den generell auftretenden altersphysiologischen Prozessen kann dann im hohen Alter zu funktionalen Einschränkungen führen. Obwohl aber steigendes Alter mit einer sinkenden funktionalen Gesundheit zusammenhängt, ist Hilfe- und Pflegebedürftigkeit, eine Konsequenz sehr eingeschränkter funktionaler Gesundheit, erst im hohen Alter stark verbreitet, in der Regel erst jenseits des 80. Lebensjahres (Menning \& Hoffmann 2009).

Die subjektive Gesundheit ist die individuelle Bewertung des eigenen Gesundheitszustandes und gibt an, wie sich Menschen gesundheitlich fühlen und ihre Einschränkungen und Krankheit(en) selbst einschätzen. Diese Gesundheitsdimension ist insbesondere deshalb von Bedeutung, weil Personen, die sich subjektiv gesund fühlen, im Durchschnitt länger leben als Personen mit einer weniger positiven subjektiven Gesundheitseinschätzung - unabhängig von ihrer körperlichen Gesundheit (Idler \& Benyamini 1997; Benyamini \& Idler 1999). Eine Erklärung dafür könnte sein, dass Personen mit subjektiv besserer Gesundheit eher gesundheitsförderliche Maßnahmen ergreifen, beziehungsweise Risikoverhaltensweisen nicht zeigen oder ändern (Benyamini 2011).

Es kann zu großen Diskrepanzen zwischen objektivem und subjektivem Gesundheitszustand kommen (Chipperfield 1993; Wettstein, Schilling, \& Wahl 2016), besonders im hohen und sehr hohen Alter. Dies zeigt sich unter anderem darin, dass viele ältere Menschen, obwohl es ihnen körperlich und funktional immer schlechter geht, aussagen, dass sie sich gesund fühlen (Spuling, Wurm, Wolff, \& Wünsche 2017a). Zwar nimmt auch die subjektive Bewertung der Gesundheit in der zwei- ten Lebenshälfte $a b$, allerdings weniger stark als es die tatsächliche Verschlechterung der körperlichen Gesundheit vermuten lassen würde (Jylhä, Guralnik, Balfour, \& Fried 2001; Wurm, Lampert, \& Menning 2009). Wenn Menschen ihre eigene Gesundheit bewerten, fließen dabei auch Faktoren jenseits der körperlichen und funktionalen Gesundheit ein, zum Beispiel das Wohlbefinden und die Lebenssituation, das Gesundheitsverhalten, die psychische Gesundheit, die individuelle Persönlichkeit und der eigene Lebensstil (Jylhä 2009). Somit ist es besonders wichtig, auch die selbsteingeschätzte Gesundheit zu berücksichtigen, um ein möglichst umfassendes Bild von der Gesundheitsentwicklung über die zweite Lebenshälfte zu bekommen.

\section{Geschlechterunterschiede in der funktionalen und subjektiven Gesundheit}

Die gesundheitliche Situation von Frauen und Männern unterscheidet sich auf vielfältige Weise. Frauen berichten durchschnittlich mehr funktionale Einschränkungen als Männer (Wolff et al. 2017). Dies kann unter anderem daran liegen, dass Frauen häufiger von Krankheiten betroffen sind, welche die Mobilität beeinflussen, zum Beispiel Arthrose und Osteoporose (Pietschmann, Rauner, Sipos, \& Kerschan-Schindl 2009). Insgesamt erleben Frauen in ihrem Lebenslauf zudem größere physiologische und hormonelle Veränderungen als Männer, verursacht unter anderem durch Schwangerschaft und Menopause. Frauen sind häufiger von Multimorbidität betroffen als Männer (Fuchs, Busch, Lange, \& Scheidt-Nave 2012), was ihr Risiko für funktionale Einschränkungen erhöht. Zudem haben auch die im Durchschnitt geringeren Bildungs- und Einkommensniveaus von Frauen aus älteren Geburtsjahrgängen einen Einfluss auf ihre Gesundheitsentwicklung. Es hat sich gezeigt, dass Gruppen mit niedrigem Einkommen und geringerer Bildung weniger Ressourcen zur Aufrechterhaltung der Gesundheit haben (z. B. finanzielle Mittel, soziale Beziehungen, Wissen über Gesund- 
heit und Gesundheitsverhalten; Lynch 2003). Diese Geschlechterunterschiede in der Gesundheit können also durchaus in geschlechtsspezifischen sozialen Ungleichheiten begründet liegen.

Ergebnisse des Deutschen Alterssurveys (DEAS) zeigen, dass Frauen und Männer ihre Gesundheit subjektiv gleich bewerten, trotz der genannten Unterschiede in der funktionalen Gesundheit (Spuling et al. 2017a). Dies könnte an sozialen Vergleichsprozessen und der dabei von Frauen und Männern genutzten Referenzgruppen liegen: Wenn sich Frauen mit anderen Frauen ihres Alters vergleichen, ist ein gewisses Maß an funktionalen Einschränkungen „normal“ - was so zu einem besseren selbsteingeschätztem Gesundheitszustand führt als wenn sie sich mit gleichaltrigen Männern vergleichen würden (Zajacova, Huzurbazar, \& Todd 2017).

\section{Kohortenunterschiede in der funktionalen und subjektiven Gesundheit von Frauen und Männern}

Geschlechterunterschiede in der Gesundheit müssen jedoch immer in den historischen Kontext eingebettet werden. Die Rolle der Geburtskohorte sollte nicht vernachlässigt werden, da sich im Laufe der letzten Jahrzehnte die Geschlechterverhältnisse in Deutschland und damit auch die gesellschaftliche Stellung der Frau gewandelt haben - Frauen später geborener Kohorten sind besser gebildet und beziehen höhere Einkommen als Frauen früher geborener Kohorten. Die Unterschiede zu den Männern werden hier geringer, so dass in nachfolgenden Kohorten auch Geschlechterunterschiede in der Gesundheit möglicherweise verschwinden oder zumindest geringer ausfallen werden (Crimmins \& Beltrán-Sánchez 2011).

Nicht nur auf Geschlechterunterschiede bezogen, sondern auch generell gibt es historische Entwicklungen, die besonders für das Gesundheitssystem, die Pflegeversicherung und Rentenpolitik wichtige Schlüsse zulassen. Insgesamt ist mit einer Verbesserung des allgemeinen Gesundheits- zustandes zu rechnen, da nachfolgende Kohorten die positiven Auswirkungen des medizinischen Fortschritts, höherer Bildungsniveaus und eines teilweise gesünderen Lebensstils im Allgemeinen $\mathrm{zu}$ spüren bekommen. Zum Beispiel funktioniert heutzutage die Früherkennung vieler Krankheiten sehr gut und insgesamt treiben Menschen mehr Sport. Es konnte anhand von Daten des Deutschen Alterssurveys gezeigt werden, dass in älteren Altersgruppen die sportliche Betätigung zwischen 2008 und 2014 anstieg und dieser positive Wandel bei Frauen besonders stark ausgeprägt war (Spuling, Ziegelmann, \& Wünsche 2017b).

In der Tat wird eine Zunahme der in guter Gesundheit verbrachten Lebensjahre erwartet (Kroll \& Ziese 2009). Weiterhin zeigen Studien, die Daten des Sozio-oekonomischen Panels nutzen, dass später geborene Kohorten weniger funktionale Einschränkungen berichten als früher geborene Kohorten (Trachte, Sperlich, \& Geyer 2015).

In Bezug auf ausschließlich die subjektive Gesundheit sind bisherige Forschungsergebnisse weniger eindeutig und komplexer, was unter anderem auf die unterschiedlichen Einflussfaktoren, die betrachtet wurden, zurückzuführen ist. Dabei finden sich Studien, die eine schlechtere subjektive Gesundheit sowohl bei später Geborenen (Chen, Cohen, \& Kasen 2007; Idler 1993), als auch bei früher Geborenen zeigen (Dening, Chi, Brayne, Huppert, Paykel, \& O'Connor 1998; Heikkinen, Kauppinen, Rantanen, Leinonen, Lyyra, Suutama, \& Heikkinen 2011), sowie Studien, die keine Kohorteneffekte finden (Hoeymans, Feskens, van den Bos, \& Kromhout 1997).

Neben dem Vergleich von Frauen und Männern hinsichtlich der Alternsverläufe ihrer funktionalen und subjektiven Gesundheit ist es also auch wichtig, diese Geschlechterunterschiede im historischen Kontext $\mathrm{zu}$ betrachten, indem die Alternsverläufe von Frauen und Männern unterschiedlicher Geburtskohorten miteinander verglichen werden. Dadurch lässt sich eine Aussage darüber treffen, ob sich im Zuge des gesellschaftlichen Wandels die Alternsverläufe in der Gesundheit von Frauen und Männern einander angenähert haben, 
oder ob die Geschlechterunterschiede in den Verläufen stabil geblieben oder sogar größer geworden sind.

\section{Fragestellungen}

Zusammenfassend werden im vorliegenden Kapitel die folgenden Forschungsfragen untersucht:

\subsection{Methoden}

\section{Stichprobe}

Für das vorliegende Kapitel wurden die Daten aller Erhebungen des Deutschen Alterssurveys (DEAS; 1996, 2002, 2008, 2011, 2014, 2017) berücksichtigt. Insgesamt gehen in die Analysen zur funktionalen Gesundheit (erstmals 2002 erfasst) 15131 Personen ein. Die Analysemodelle zur subjektiven Gesundheit (erstmals 1996 erfasst) berücksichtigen dagegen 20017 Personen.

\section{Indikatoren}

Funktionale Gesundheit. Die funktionale Gesundheit wird mit Hilfe von zehn Fragen zu Einschränkungen in täglichen Aktivitäten (Subskala körperliche Funktionsfähigkeit der SF-36-Skala zu gesundheitsbezogener Lebensqualität; Bullinger \& Kirchberger 1998) im Rahmen des persönlichen Interviews erfasst. Die Befragten werden dazu aufgefordert, anhand einer dreistufigen Rating-Skala anzugeben, ob sie bei den folgenden Alltagstätigkeiten durch ihren derzeitigen Gesundheitszustand „stark eingeschränkt", „etwas eingeschränkt“ oder „überhaupt nicht eingeschränkt" sind: Anstrengende Tätigkeiten (z.B. schnell laufen, schwere Gegenstände heben, anstrengenden Sport treiben); mittelschwere Tätigkeiten (z.B. einen Tisch verschieben, staubsaugen, kegeln, Golf spielen); Einkaufstaschen heben oder tragen; mehrere Trep-
1) Unterscheiden sich Frauen und Männer hinsichtlich ihrer Alternsverläufe funktionaler und subjektiver Gesundheit?

2) Verändern sich Geschlechterunterschiede in den Alternsverläufen funktionaler und subjektiver Gesundheit über verschiedene Geburtskohorten hinweg?

penabsätze steigen; sich beugen, knien, bücken; mehr als einen Kilometer zu Fuß gehen; mehrere Straßenkreuzungen weit zu Fuß gehen; eine Straßenkreuzung weit zu Fuß gehen; sich baden oder anziehen. Die Antworten wurden über jede Person summiert und so transformiert, dass die SkalaAusprägungen zwischen null und 100 liegt, wobei höhere Werte eine geringere Einschränkung und somit eine bessere funktionale Gesundheit bedeuten. In Anlehnung an Nejati und Kollegen (2008) wird bei der Ergebnisdarstellung folgende Kategorisierung als Interpretationshilfe herangezogen: Sehr gute funktionale Gesundheit (Werte 80-100), gute funktionale Gesundheit (60-79), moderate funktionale Gesundheit (40-59), schlechte funktionale Gesundheit (20-39), sehr schlechte funktionale Gesundheit (0-19).

Subjektive Gesundheit. Die subjektive Gesundheit wird im Rahmen des persönlichen Interviews erfasst. Alle Befragten werden aufgefordert, ihren derzeitigen Gesundheitszustand auf einer fünfstufigen Rating-Skala zu bewerten. Die vorgegebenen Antwortalternativen lauteten dabei „sehr gut“, „gut“, „mittel“, „schlecht“, „sehr schlecht“. Höhere Werte - auf einer Skala von 1 „sehr schlecht“ bis 5 ,sehr gut“ - entsprechen einer besseren subjektiven Gesundheit.

Gruppierungsvariablen. Alternsverläufe funktionaler und subjektiver Gesundheit werden zwischen Frauen und Männern sowie zwischen verschiedenen Geburtskohorten verglichen. Zur Beschrei- 
bung von Kohortenunterschieden werden drei ausgewählte Geburtskohorten betrachtet: 1930-1939 Geborene, 1940-1949 Geborene, 1950-1959 Geborene. Diese Auswahl führt dazu, dass sich die Stichprobengröße bei den Analysen zu Kohortenunterschieden auf 10677 Personen bei der funktionalen Gesundheit verringert und auf 13929 Personen bei der subjektiven Gesundheit.

Kontrollvariablen. Als zusätzliche Kontrollvariable dient in allen Analysen die Region (West-/Ostdeutschland) als zentrale Stichprobenvariable des Deutschen Alterssurveys.

\section{Statistische Analyseverfahren}

Mögliche Verzerrungen der Analysen, die sich aus dem selektiven Ausfall von Personen im Längsschnitt ergeben könnten, wurden mit der Full Information Maximum Likelihood Methode ausgeglichen. In die Analysen konnten dadurch auch jene Personen einbezogen werden, die nur einmal am Deutschen Alterssurvey teilgenommen haben. Die Alternsverläufe von funktionaler und sub-

\subsection{Ergebnisse}

\subsubsection{Funktionale Gesundheit im Verlauf der zweiten Lebenshälfte}

Alternsverläufe der funktionalen Gesundheit unterscheiden sich zwischen Frauen und Männern: Frauen berichten über die gesamte zweite Lebenshälfte im Durchschnitt mehr Einschränkungen als Männer.

Die funktionale Gesundheit wird mit zunehmendem Alter im Durchschnitt schlechter. Das heißt funktionale Einschränkungen nehmen zu, je älter Frauen und Männer werden. Insgesamt zeigen sich Geschlechterunterschiede - sowohl im Ausgangsniveau, das wir im Alter von Anfang 40 Jahren betrachten, als auch hinsichtlich der Abnahme jektiver Gesundheit wurden mit Hilfe von Dual Change Score Modellen (McArdle 2001; McArdle \& Hamagami 2001) untersucht. In solchen Modellen werden individuelle Alternsverläufe geschätzt und man kann Unterschiede im Ausgangsniveau, also hier im Alter von 40 Jahren, sowie Unterschiede im Ausmaß von Veränderungen, also hier im Alter zwischen 40 und 90 Jahren, untersuchen und beschreiben. Für diese Veränderung gibt es einen zu schätzenden linearen sowie einen nichtlinearen Anteil.

Ob sich Frauen und Männer hinsichtlich der Alternsverläufe funktionaler und subjektiver Gesundheit unterscheiden, wurde untersucht, indem der Effekt der Variable Geschlecht auf Ausgangsniveau und Veränderung von subjektiver und funktionaler Gesundheit auf Signifikanz getestet wurde. Um Geschlechterunterschiede hinsichtlich der Kohortenunterschiede zu untersuchen, wurde zusätzlich der Effekt der Interaktion zwischen den Variablen Kohorte und Geschlecht auf Ausgangsniveau und Veränderung auf Signifikanz getestet. Das Signifikanzniveau wurde bei allen Analysen auf $\mathrm{p}<0,05$ festgesetzt.

der funktionalen Gesundheit über die zweite Lebenshälfte hinweg. Wie Abbildung 3-1 zeigt, berichten Frauen über den insgesamt betrachteten Alternsverlauf von gut 50 Jahren eine schlechtere funktionale Gesundheit als Männer. Dieser Geschlechterunterschied nimmt mit steigendem Alter zu: Während sich Frauen und Männer im Alter von 40 Jahren nur gering in ihrer funktionalen $\mathrm{Ge}$ sundheit unterscheiden (geschätzte mittlere Differenz auf der Skala von null bis 100: 1,6 Punkte), nehmen funktionale Einschränkungen bei Frauen mit zunehmendem Alter sehr viel stärker zu als es bei Männern der Fall ist (geschätzte mittlere Differenz am Ende des achten Lebensjahrzehnts: rund 13 Punkte). Diese stärkere Abnahme der funktionalen Gesundheit führt dazu, dass sich Frauen und 
Abbildung 3-1 Funktionale Gesundheit bei Frauen und Männern im Alternsverlauf

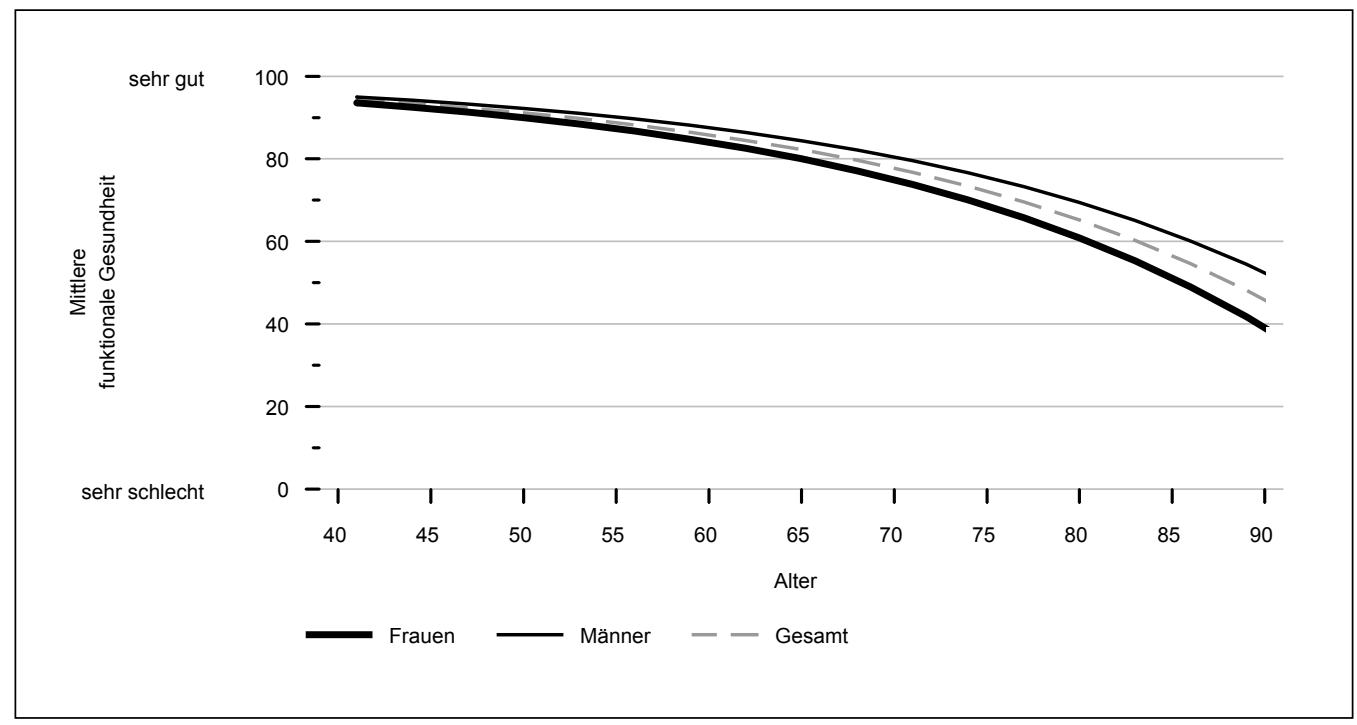

Quelle: DEAS 2002-2017, Schätzungen basieren auf $n=15131$ Studienteilnehmenden mit insgesamt $n=28882$ Beobachtungen; kontrolliert für Region; Kategorisierung der Werte nach Nejati et al. (2008): sehr gute funktionale Gesundheit (80-100), gute funktionale Gesundheit (60-79), moderate funktionale Gesundheit (40-59), schlechte funktionale Gesundheit (20-39), sehr schlechte funktionale Gesundheit (o-19).

Unterschiede zwischen Frauen und Männern im geschätzten Ausgangsniveau (Alter Anfang 40 Jahre) sind signifikant - ebenso unterscheidet sich der geschätzte Verlauf zwischen Frauen und Männern signifikant. Frauen haben im Durchschnitt eine etwas schlechtere funktionale Gesundheit im Alter von 40 Jahren und die Abnahme mit zunehmendem Alter fällt bei Frauen im Durchschnitt stärker aus als bei Männern. Folglich vergrößern sich Geschlechterunterschiede hinsichtlich der funktionalen Gesundheit mit zunehmendem Alter.

Männer im hohen Alter sehr viel stärker in ihrer funktionalen Gesundheit unterscheiden als es im mittleren Erwachsenenalter der Fall ist. Bei Frauen wechselt ihre durchschnittliche funktionale Gesundheit ab einem Alter von ca. 65 Jahren vom Bereich „sehr gut" zu "gut" und ab einem Alter von ca. 81 Jahren von "gut" (Werte zwischen 60 und 79) zu „moderat“ (Werte zwischen 40 und 59). Bei Männern erfolgen diese Wechsel jeweils später im Alternsverlauf: Von „sehr gut“ zu „gut“ (Werte zwischen 60 und 79) durchschnittlich in einem Alter von etwa 70 Jahren, und von "gut“ zu „moderat" durchschnittlich in einem Alter von etwa 86 Jahren. Die beschriebenen Übergänge in der funktionalen Gesundheit finden bei Männern im Durchschnitt also jeweils ca. fünf Jahre später statt als bei Frauen. Männer erreichen im Durchschnitt auch im sehr hohen Alter nicht den Wertebereich von „schlechter" funktionaler Gesundheit (Werte zwischen 20 und 39), und bei Frauen vollzieht sich dieser Übergang erst im Übergang vom 89. zum 90. Lebensjahr. Die durchschnittliche funktionale Gesundheit der Frauen sinkt also selbst in diesem sehr hohen Alter nicht in den Bereich „sehr schlecht" (Werte zwischen 0 und 19), und bei den Männern ist sie im Durchschnitt auch im sehr hohen Alter noch „moderat“.

Für die funktionale Gesundheit zeigen sich Kohortenunterschiede: Frauen und Männer später geborener Kohorten werden vermutlich mit einer besseren funktionalen Gesundheit ins hohe und sehr hohe Al- 
ter kommen als Frauen und Männer früher geborener Kohorten.

Sowohl im geschätzten durchschnittlichen Ausgangsniveau im mittleren Erwachsenenalter als auch hinsichtlich der Stärke der durchschnittlichen Abnahme mit zunehmendem Alter zeigen sich Kohortenunterschiede in der funktionalen Gesundheit (Abbildung 3-2). Während im mittleren Erwachsenenalter die früher geborene Kohorte (in den Jahren 1930-1939 Geborene) im Durchschnitt eine bessere funktionale Gesundheit im Sinne weniger berichteter Einschränkungen aufweist als die beiden später geborenen Kohorten (gemäß unserer Modellschätzung, denn die Gesundheit der 1930 bis 1939 Geborenen wurde im
Alter von 40 Jahren im Rahmen des Deutschen Alterssurveys (DEAS) nicht erfasst), ist die Abnahme bis zum Ende des achten Lebensjahrzehnts in dieser Kohorte am stärksten ausgeprägt. Mit anderen Worten: Der Vorteil früherer Geburtskohorten gegenüber später Geborenen hinsichtlich der funktionalen Gesundheit ist also nur im mittleren Erwachsenenalter zu beobachten und dreht sich dann im Alter von ca. 70 Jahren zugunsten späterer Geburtsjahrgänge um. Die am spätesten geborene hier betrachtete Kohorte (1950-1959 Geborene) hat im mittleren Erwachsenenalter eine etwas schlechtere funktionale Gesundheit als die beiden anderen Geburtskohorten, verzeichnet jedoch eine geringere Abnahme der funktionalen Gesundheit mit zunehmendem Alter im Vergleich zu 1940 bis

\section{Abbildung 3-2 Funktionale Gesundheit nach Kohorten im Alternsverlauf}

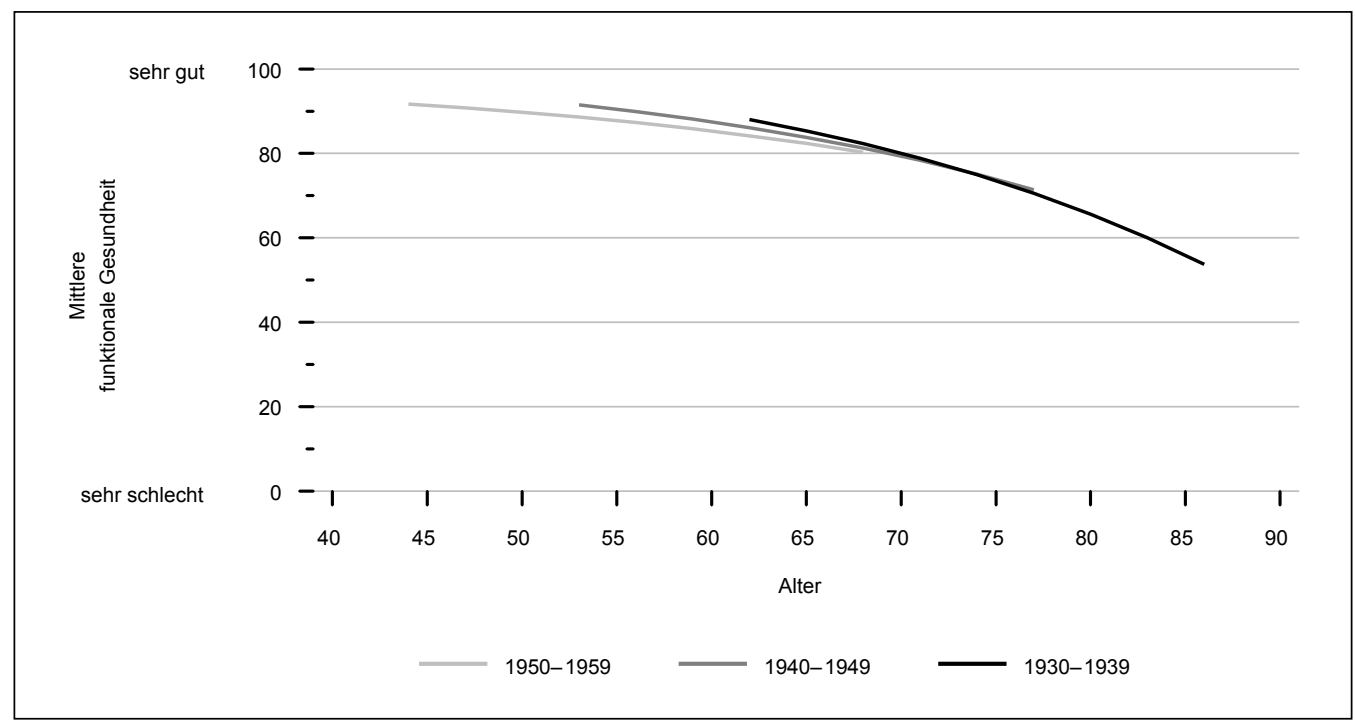

Quelle: DEAS 2002-2017, Schätzungen basieren auf $n=10677$ Studienteilnehmenden mit insgesamt $n=21146$ Beobachtungen; kontrolliert für Region und Geschlecht; der geschätzte Alternsverlauf für die funktionale Gesundheit in der Geburtskohorte 1950-1959 beginnt erst ab einem Alter von 43 Jahren, da die funktionale Gesundheit erst seit 2002 Bestandteil des DEAS-Instruments ist; Kategorisierung der Werte nach Nejati et al. (2008): sehr gute funktionale Gesundheit (80-100), gute funktionale Gesundheit (60-79), moderate funktionale Gesundheit (40-59), schlechte funktionale Gesundheit (20-39), sehr schlechte funktionale Gesundheit (o-19).

Unterschiede zwischen den Geburtskohorten im geschätzten Ausgangsniveau (Alter Anfang 40 Jahre) sind signifikant - ebenso unterscheidet sich der geschätzte Verlauf zwischen den Geburtskohorten signifikant. Später Geborene haben eine schlechtere funktionale Gesundheit im mittleren Erwachsenenalter im Vergleich zu früher Geborenen (wenn man den Verlauf der früher geborenen Kohorten bis ins mittlere Erwachsenenalter weiterführt), dafür fällt die Abnahme mit zunehmendem Alter bei später Geborenen schwächer aus als bei früher Geborenen. 
1949 und 1930 bis 1939 Geborenen. Es kann folglich erwartet werden, dass die später geborenen Kohorten mit einer besseren funktionalen Gesundheit im Sinne weniger berichteter funktionaler Einschränkungen ins hohe und sehr hohe Alter kommen werden als früher geborene Kohorten. Diese Kohortenunterschiede treffen gleichermaßen auf Frauen und Männer zu (vgl. Abbildung 3-3).

Dieser Kohorteneffekt trifft gleichermaßen auf Frauen und Männer zu. Das heißt, die Abnahme der funktionalen Gesundheit mit zunehmendem Alter fällt in früher geborenen Kohorten gleichermaßen bei Frauen und Männern stärker aus als es bei Frauen und Männern später geborener Kohorten der Fall ist, und die Geschlechterdiskrepanz im Verlauf unterscheidet sich nicht signifikant zwischen den Kohorten.

\subsubsection{Subjektive Gesundheit im Verlauf der zweiten Lebenshälfte}

Alternsverläufe der subjektiven Gesundheit unterscheiden sich nicht zwischen Frauen und Männern: Frauen und Männer bewerten gleichermaßen ihre Gesundheit mit zunehmendem Alter schlechter.

Sowohl im Ausgangsniveau (im mittleren Erwachsenenalter) als auch in der Veränderung der subjektiven Gesundheit im Verlauf der zweiten Lebenshälfte unterscheiden sich Frauen und Männer nicht. In Abbildung 3-4 ist der allgemeine durchschnittliche Alternsverlauf der subjektiven Gesundheit verdeutlicht sowie der durchschnittliche Alternsverlauf für Frauen und Männer getrennt. Wie in Abbildung 3-4 dargestellt, überlappen sich die drei Linien. Das heißt, Frauen und Männer unterscheiden sich weder im mittleren Erwachsenenalter, noch wenn sie älter werden, signifikant in ihrer subjektiven Gesundheitsbewertung. Im mittleren Erwachsenenalter (Anfang des fünften Lebensjahrzehnts) bewerten Menschen ihre Gesundheit auf der fünfstufigen Antwortskala im Durchschnitt mit dem Wert 3,8 („gut“); wenn Frauen und Männer das Ende des neunten Lebens- jahrzehnts und somit das sehr hohe Alter erreichen, liegt der durchschnittliche Wert ihrer subjektiven Gesundheitsbewertung immer noch bei 2,9 („mittel“).

Für die subjektive Gesundheit zeigen sich keine Kohortenunterschiede: Frauen und Männer späterer Geburtsjahrgänge unterscheiden sich nicht im Ausgangsniveau und im Alternsverlauf ihrer Gesundheitsbewertung von Frauen und Männern früherer Geburtsjahrgänge.

Hinsichtlich der subjektiven Gesundheit sind keine Kohortenunterschiede beobachtbar - weder bei Frauen noch bei Männern (Abbildung 3-5). Der durchschnittliche Alternsverlauf der subjektiven Gesundheit getrennt für die drei betrachteten Geburtskohorten verläuft ähnlich wie der allgemeine durchschnittliche Verlauf der subjektiven Gesundheit (Abbildung 3-4). Der allgemeine durchschnittliche Alternsverlauf der subjektiven Gesundheit trifft also nicht nur gleichermaßen auf Frauen und Männer zu, sondern auch gleichermaßen auf alle drei betrachteten Geburtskohorten (1930-1939 Geborene; 1940-1949 Geborene; 19501959 Geborene). 
Abbildung 3-3 Funktionale Gesundheit bei Frauen und Männern nach Kohorten im Alternsverlauf

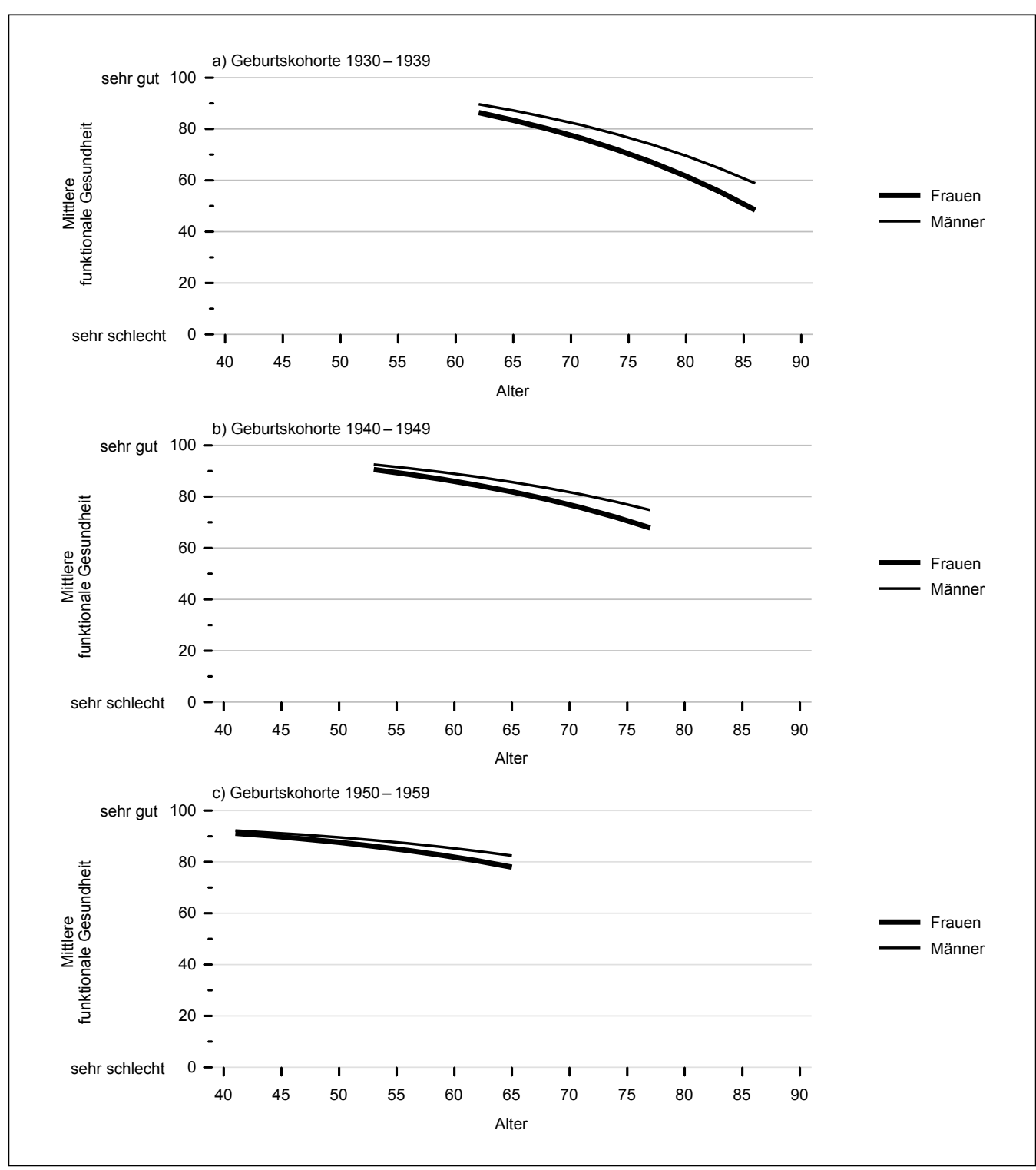

Quelle: DEAS 2002-2017, Schätzungen basieren auf $n=10677$ Studienteilnehmenden mit insgesamt $n=21146$ Beobachtungen; kontrolliert für Region; die geschätzten Alternsverläufe für die funktionale Gesundheit in der Geburtskohorte 1950-1959 beginnen erst ab einem Alter von 43 Jahren, da die funktionale Gesundheit erst seit 2002 Bestandteil des DEAS-Instruments sind; Kategorisierung der Werte nach Nejati et al. (2008): sehr gute funktionale Gesundheit (80-100), gute funktionale Gesundheit (6o-79), moderate funktionale Gesundheit (40-59), schlechte funktionale Gesundheit (20-39), sehr schlechte funktionale Gesundheit (o-19).

Kohortenunterschiede hinsichtlich der funktionalen Gesundheit treffen auf Frauen und Männer gleichermaßen zu: Die Abnahme der funktionalen Gesundheit mit steigendem Alter fällt bei später geborenen Frauen und Männern schwächer aus als bei früher geborenen Frauen und Männern. Geschlechterunterschiede zeigen sich gleichermaßen in allen drei betrachteten Kohorten. 
Abbildung 3-4 Subjektive Gesundheit bei Frauen und Männern im Alternsverlauf

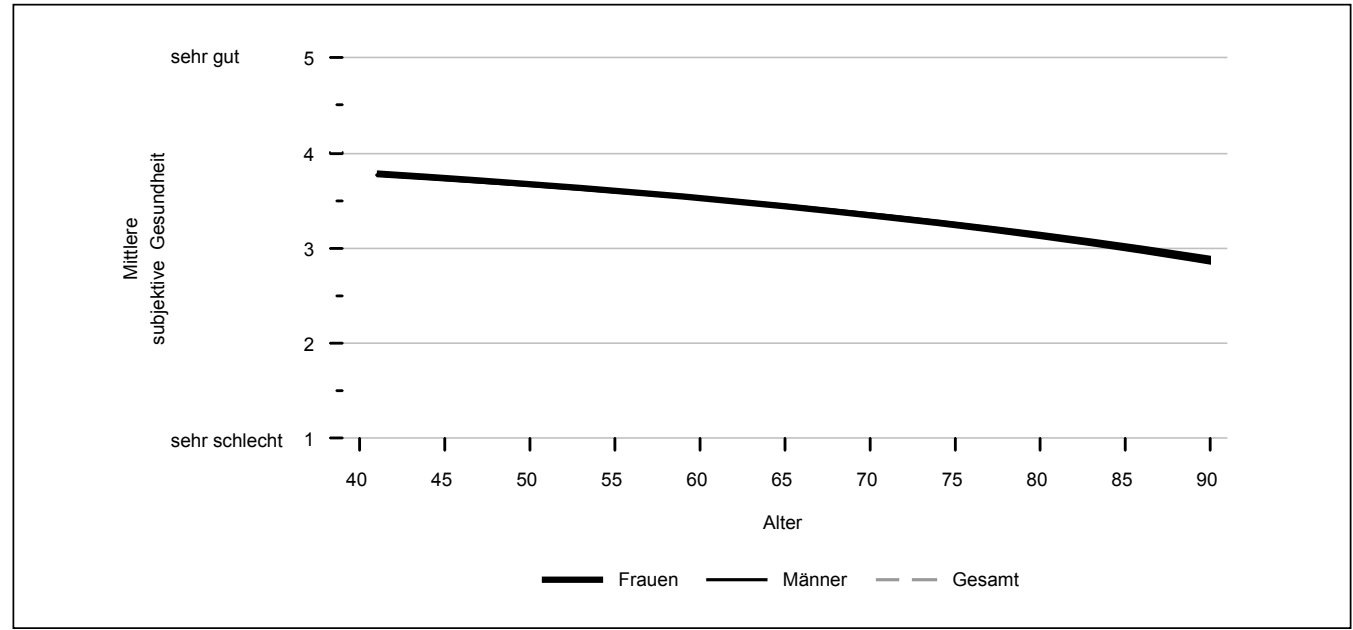

Quelle: DEAS 1996-2017, Schätzungen basieren auf $\mathrm{n}=20017$ Studienteilnehmenden mit insgesamt $\mathrm{n}=38893$ Beobachtungen; kontrolliert für Region und Geschlecht; 1 („sehr schlechte subjektive Gesundheit“), 2 („schlechte subjektive Gesundheit“), 3 („mittlere subjektive Gesundheit“), 4 („gute subjektive Gesundheit“), 5 („sehr gute subjektive Gesundheit“).

Unterschiede zwischen Frauen und Männern hinsichtlich ihrer subjektiven Gesundheit im geschätzten Ausgangsniveau (Alter Anfang 40 Jahre) und im geschätzten Verlauf sind statistisch nicht signifikant. Die Abnahme der subjektiven Gesundheit mit zunehmendem Alter erfolgt für Frauen und Männer gleichermaßen nicht linear.

\section{Abbildung 3-5 Subjektive Gesundheit nach Kohorten im Alternsverlauf}

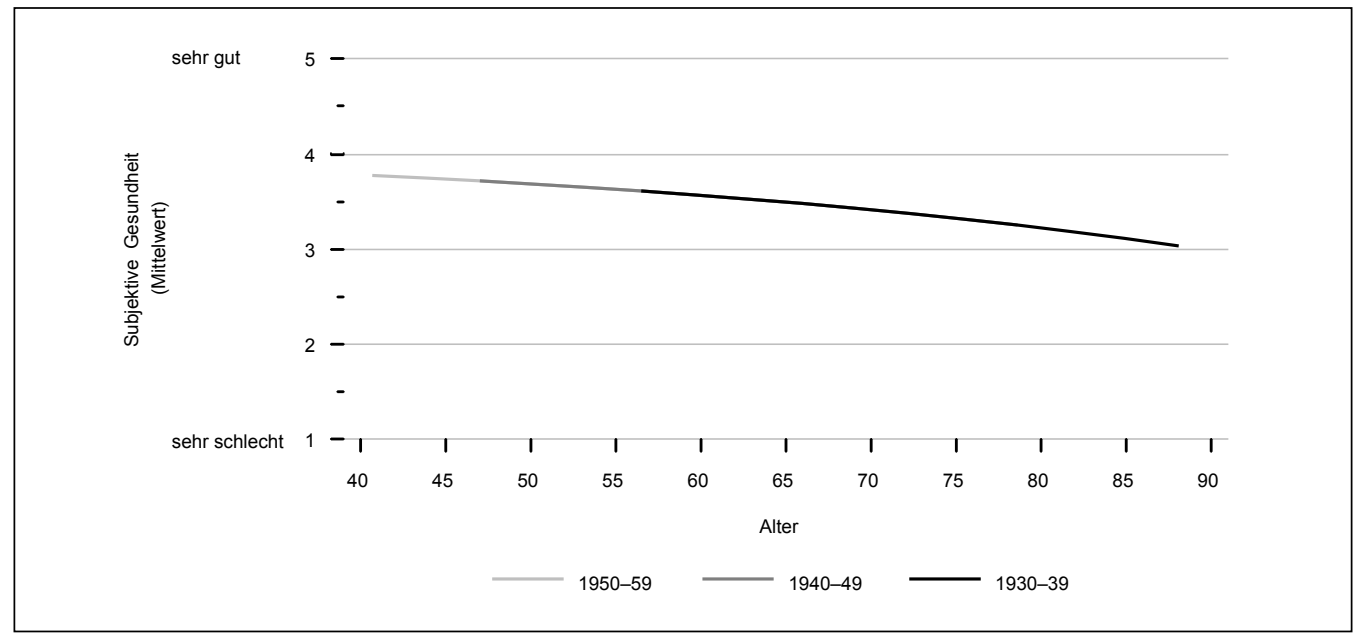

Quelle: DEAS 1996-2017, Schätzungen basieren auf $n=13929$ Studienteilnehmenden mit insgesamt $\mathrm{n}=28887$ Beobachtungen; kontrolliert für Region und Geschlecht; 1 („sehr schlechte subjektive Gesundheit“), 2 („,schlechte subjektive Gesundheit“), 3 („mittlere subjektive Gesundheit“), 4 („gute subjektive Gesundheit“), 5 („sehr gute subjektive Gesundheit“).

Unterschiede zwischen den Geburtskohorten im geschätzten Ausgangsniveau (Alter Anfang 40 Jahre) sowie im geschätzten Verlauf sind nicht signifikant. 


\subsection{Diskussion}

\section{Zusammenfassung}

Im Allgemeinen nehmen sowohl die funktionale als auch die subjektive Gesundheit der Frauen und Männer über die zweite Lebenshälfte ab. Geschlechterunterschiede zeigen sich in diesem $\mathrm{Zu}$ sammenhang ausschließlich bei Betrachtung der Alternsverläufe der funktionalen Gesundheit: Sowohl Ausgangsniveau als auch Abnahme mit zunehmendem Alter fallen für Frauen negativer aus als für Männer. Weiterhin gibt es Kohortenunterschiede in den Alternsverläufen der funktionalen Gesundheit, die auf Frauen und Männer gleichermaßen zutreffen: Früher geborene Kohorten haben eine bessere funktionale Gesundheit im mittleren Erwachsenenalter als später geborene Kohorten. Später geborene Kohorten weisen dagegen eine schwächere Abnahme der funktionalen Gesundheit mit zunehmendem Alter auf und für sie wird deshalb eine bessere funktionale $\mathrm{Ge}$ sundheit im späten Erwachsenenalter erwartet als bei früher geborenen Kohorten. In der Einschätzung ihrer subjektiven Gesundheit und in der Veränderung dieser Einschätzung mit zunehmendem Alter unterscheiden sich Frauen und Männer dagegen nicht. Obwohl Frauen also mehr funktionale Einschränkungen berichten als Männer und zusätzlich eine stärkere Abnahme der funktionalen Gesundheit mit zunehmendem Alter erleben als Männer, unterscheiden sie sich nicht von Männern in ihrer subjektiven Gesundheit und deren Veränderung mit zunehmendem Alter.

\section{Interpretation}

Die funktionale Gesundheit geht besonders im höheren Alter deutlich zurück, bei der subjektiven Gesundheit lassen sich dagegen nur leichte Einbußen feststellen. Dies unterstreicht die Multidimensionalität der gesundheitlichen Situation von Personen - Gesundheit umfasst verschiedene Aspekte, die sich im Alternsverlauf unterschied- lich entwickeln können. Ältere Personen tendieren dazu, sich gesundheitlich mit Gleichaltrigen $\mathrm{zu}$ vergleichen (Leinonen, Heikkinen, \& Jylhä 2001), was zu einer subjektiv positiveren Bilanz führt als der Vergleich mit jüngeren Altersgruppen. Ältere Menschen passen zudem ihre eigenen Standards und Erwartungen hinsichtlich ihrer Gesundheit an Veränderungen ihres körperlichen und funktionalen Gesundheitszustandes an, ein Phänomen, das in der Literatur als Response Shift bezeichnet wird (Sprangers \& Schwartz 1999). So wird eine positive subjektive Bewertung der eigenen Gesundheit begünstigt (Galenkamp, Huisman, Braam, \& Deeg 2012; Spuling, Wolff, \& Wurm 2017a). Das bedeutet, dass ältere Menschen im Allgemeinen eine Verschlechterung ihrer Gesundheit erwarten und diese als „normal“ bewerten. Das heißt wiederum, dass eine Verschlechterung des körperlichen oder funktionalen Gesundheitszustandes nicht unbedingt mit einer Veränderung der subjektiven Bewertung der Gesundheit einhergehen muss.

Alternsverläufe funktionaler und subjektiver Gesundheit von Frauen und Männern: Bei Betrachtung der funktionalen Gesundheit zeigen sich Geschlechterunterschiede: Frauen sind gegenüber Männern im Alter von 40 Jahren häufiger von funktionalen Einschränkungen betroffen, sie haben also eine etwas geringere funktionale Gesundheit. Dieser Geschlechterunterschied wird im Laufe des Alterns größer, da Frauen stärkere Einbußen bei der funktionalen Gesundheit erleiden als Männer. Dies kann daran liegen, dass Frauen eine höhere Lebenserwartung haben als Männer und infolge der höheren Lebenserwartung mehr Frauen als Männer trotz gesundheitlicher Einbußen ein hohes Alter erreichen. Die hier beschriebenen Geschlechterunterschiede über die gesamte zweite Lebenshälfte hinweg könnten darüber hinaus eventuell aber auch durch Bildungsunterschiede zwischen Frauen und Männern erklärt werden. Bildung spielt eine wichtige Rolle für körperliche und funktionale Gesundheitsverläufe (Lynch 2003; Schöllgen, Huxhold, \& Tesch-Römer 2010), da eine 
höhere Bildung über unterschiedliche Mechanismen mit einem insgesamt gesundheitsförderlicheren Leben zusammenhängt. Auch geschlechtsspezifische Ungleichheiten in der Arbeitswelt, welche auch in der heutigen Zeit noch fortwähren, können die Unterschiede in der funktionalen Gesundheit von Frauen und Männern möglicherweise erklären. Ein höheres Einkommen, begünstigt durch höhere Bildung, ermöglicht einen gesünderen Lebensstil und eine bessere medizinische Versorgung.

Hinsichtlich der subjektiven Gesundheit zeigt sich kein Geschlechterunterschied - weder im Ausgangsniveau im Alter von 40 Jahren noch im Verlauf des Älterwerdens. Wenn man dabei noch die im Allgemeinen schlechtere psychische Gesundheit von Frauen im Alter (zumindest hinsichtlich depressiver Symptome; siehe Kapitel 4 Lebenszufriedenheit und depressive Symptome in diesem Band) hinzuzieht, ergibt sich ein interessantes Bild - trotz größerer funktionaler Einschränkungen und stärkerer depressiver Symptome, zeigen Frauen gegenüber Männern keinen schlechteren subjektiven Gesundheitszustand und mit zunehmendem Alter keinen stärkeren Rückgang in der subjektiven Gesundheit. Dafür gibt es unterschiedliche Erklärungsansätze: Es kann zum Beispiel sein, dass Frauen ein anderes Gesundheitsverständnis haben als Männer. Das bedeutet, dass bei Frauen möglicherweise zusätzliche oder andere Faktoren in die selbstbeurteilte Gesundheit einfließen beziehungsweise dass bei Frauen die funktionale Gesundheit weniger eng mit der subjektiven Gesundheitsbewertung zusammenhängt als bei Männern. Außerdem ist die subjektive Gesundheit auch stark vom sozialen Vergleichsrahmen abhängig - Frauen und Männer vergleichen ihre eigene Gesundheit nicht nur mit Gleichaltrigen, sondern auch mit Gleichaltrigen desselben Geschlechts (Wills 1992).

Alternsverläufe funktionaler und subjektiver Gesundheit bei verschiedenen Geburtskohorten: Für verschiedene Geburtsjahrgänge zeigen sich unterschiedliche Verläufe in der funktionalen Gesundheit. Früher geborene Kohorten (1930-1939) weisen eine bessere funktionale Gesundheit im mittleren Erwachsenenalter auf als später geborene Kohorten (1940-1949 und 1950-1959). Dafür nimmt die funktionale Gesundheit für die früher geborenen Kohorten aber stärker ab als für die später geborenen Kohorten. Im hohen Alter haben dementsprechend später geborene eine bessere funktionale Gesundheit als früher geborene Jahrgänge. Auch für die Analysen dieses Kapitels gilt dabei, dass sich diese in die Zukunft gerichteten Verlaufsschätzungen für die später geborenen Kohorten - die aktuell noch nicht das (sehr) hohe Alter erreicht haben - als unzutreffend erweisen können. Ihnen liegt die Annahme zugrunde, dass sich die bislang beobachteten Trends zukünftig fortsetzen werden.

Eine mögliche Erklärung für die Unterschiede im mittleren Erwachsenenalter ist hier der sich im Allgemeinen wandelnde Lebensstil: Je später eine Person geboren wird, desto höher ist die Risikowahrscheinlichkeit für Übergewicht und damit verbundene Erkrankungen wie Diabetes oder Herz-Kreislauf-Erkrankungen (Prentice 2006). Zusätzlich scheint das mittlere Erwachsenenalter - gerade in den später geborenen Kohorten - eine belastungsreiche Lebensphase zu sein, die eine potenzielle Gefahr für die sogenannte Work-Life-Balance darstellt. Anforderungen und Stressoren aus unterschiedlichen Lebensbereichen wie Familie und Arbeit treffen aufeinander, wobei Interessenund Zielkonflikte entstehen können (Knecht, Bauer, Gutzwiller, \& Hämmig 2011). Diese Stressoren können sich auf die Gesundheit auswirken. Gleichzeitig haben sich aber auch die medizinischen Möglichkeiten weiterentwickelt, welche den jüngeren Jahrgängen schon einen längeren Teil ihres Lebens zur Verfügung stehen werden als älteren Jahrgängen. Damit können die Folgen einer schlechten funktionalen Gesundheit im mittleren Erwachsenenalter im Hinblick auf die Entwicklung dieser womöglich besser „abgepuffert“ werden als in früher geborenen Kohorten - Erkrankungen werden früher erkannt und erfolgversprechender behandelt, somit werden mögliche funktionale Einschränkungen und eine daraus resultierende 
negativere Gesundheitsentwicklung über die zweite Lebenshälfte vermieden.

Diese Kohortenunterschiede stellen sich sowohl für Frauen als auch für Männer gleichermaßen dar. Dies bedeutet wiederum, dass die bereits herausgestellten Unterschiede zwischen Frauen und Männern in allen untersuchten Geburtskohorten auf ähnliche Weise zum Tragen kommen. Sie sind bislang also nicht im Zuge des gesellschaftlichen Wandels kleiner geworden.

Währenddessen scheint es bei der subjektiven Gesundheit keine Kohortenunterschiede zu geben, das heißt, dass die Abnahme über die Lebensspanne für die betrachteten Geburtsjahrgänge gleich bleibt. Der positive Kohortentrend im Alternsverlauf der funktionalen Gesundheit spiegelt sich also nicht in dem allgemeinen Alternsverlauf der subjektiven Gesundheit wider. Dieses Ergebnis unterstreicht, wie wichtig es ist, verschiedene Bereiche der Gesundheit parallel zu betrachten und zu untersuchen, da sich Veränderungen zwischen den Gesundheitsbereichen unterscheiden können. Dass sich die subjektive Gesundheit im Alternsverlauf nicht signifikant zwischen den Kohorten unterscheidet, könnte daran liegen, dass später geborene Frauen und Männer ein anderes, breiteres Verständnis von Gesundheit haben (z. B. Spuling, Wurm, Tesch-Römer, \& Huxhold 2015) und eventuell höhere Ansprüche an ihre Gesundheit stellen (z. B. Jylhä 2009).

\section{Politische Implikationen}

Für Gesundheitsversorgung und Gesundheitsförderungsmaßnahmen lassen sich aufgrund der Befunde wichtige Schlüsse ziehen. Personen im hohen und sehr hohen Alter gehören allgemein zur Risikogruppe in Bezug auf Einbußen der (funktionalen) Gesundheit (Baltes \& Smith 2003). Eine gute funktionale Gesundheit ist jedoch eine wesentliche Voraussetzung vor allem für Lebensqualität, eine selbstständige Lebensführung aber auch um Aktivitäten nachgehen zu können, die einem Freude bereiten, beispielsweise Zeit mit den En- kelkindern verbringen zu können oder ein Ehrenamt auszuüben. Besonderes Augenmerk sollte hier - neben der Risikogruppe alter und sehr alter Menschen - auf Frauen im Alter gelegt werden. Diese zeigen durchschnittlich eine durchgehend schlechtere funktionale Gesundheit in der zweiten Lebenshälfte sowie eine stärkere Abnahme im Alternsverlauf als Männer. Das heißt, dass Personen im hohen Alter und dabei besonders Frauen von maßgeschneiderten Interventionen profitieren könnten.

Zudem ist der Befund, dass auch in den später geborenen Kohorten der durchschnittliche Alternsverlauf der funktionalen Gesundheit bei Frauen negativer ausfällt als bei Männern, Anlass, über geschlechtsspezifische Interventionsmaßnahmen nachzudenken. Der gesellschaftliche Wandel hat also offenbar nicht oder noch nicht dazu beigetragen, diese Geschlechterdiskrepanz zu reduzieren, so dass es umso wichtiger ist, Maßnahmen zu ergreifen und Rahmenbedingungen zu schaffen, damit nicht auch künftige Kohorten von Frauen mit einer im Vergleich zu Männern eingeschränkteren Gesundheit ins hohe und sehr hohe Alter kommen. Während eine Angleichung von Frauen und Männern im Bereich der Bildung bereits erfolgreich erreicht werden konnte und dies vermutlich positiv auf die Geschlechterunterschiede in der funktionalen Gesundheit künftiger Kohorten wirken wird, ist es zudem wünschenswert, dass noch fortwährende geschlechtsspezifische Differenzen in der Arbeitswelt und in der Vereinbarkeitsproblematik hinsichtlich der Anforderungen aus dem Berufs- und Familienleben ebenfalls angegangen werden, um so eine nachhaltige Verbesserung der funktionalen Gesundheit für Frauen künftiger Kohorten zu erreichen.

Ein weiterer Ansatzpunkt ist die Lebensphase im mittleren Erwachsenenalter: Später geborene Kohorten haben zum einen vermutlich weniger funktionale Einschränkungen im hohen Alter, was unter anderem durch den medizinischen Fortschritt und ein besseres Gesundheitssystem begründet werden kann. Zum anderen zeigen diese später geborenen Kohorten jedoch eine geringe- 
re funktionale Gesundheit im mittleren Erwachsenenalter als früher geborene Kohorten. Dies kann auf einen teilweise ungesünder werdenden Lebensstil (Faktoren wie Übergewicht oder Tabakkonsum), aber auch eine über die Kohorten zunehmende Anhäufung von Stressoren im mittleren Erwachsenenalter, zurückzuführen sein (siehe Kapitel 6 Unbezahlte Sorgetätigkeiten in diesem
Band). Dementsprechend könnten hier Präventionsmaßnahmen zu Gesundheitsverhalten und Stressmanagement gerade für Menschen im mittleren Erwachsenenalter wichtig sein, um funktionalen Einschränkungen und einer Fortsetzung des negativen Kohortentrends in dieser Altersphase entgegenzuwirken.

\section{Literatur}

Baltes, M. M., Maas, I., Wilms, H.-U., \& Borchelt, M. (2010). Alltagskompetenz im Alter: Theoretische Überlegungen und empirische Befunde. In: U. Lindenberger, J. Smith, K. U. Mayer \& P. B. Baltes (Hrsg.) Die Berliner Altersstudie (S. 549-566). Berlin: Akademie-Verlag.

Baltes, P. B., \& Smith, J. (2003). New frontiers in the future of aging: From successful aging of the young old to the dilemmas of the fourth age. Gerontology, 49(2), 123-135.

Barnett, K., Mercer, S. W., Norbury, M., Watt, G., Wyke, S., \& Guthrie, B. (2012). Epidemiology of multimorbidity and implications for health care, research, and medical education: a cross-sectional study. The Lancet, 380(9836), 37-43.

Benyamini, Y. (2011). Why does self-rated health predict mortality? An update on current knowledge and a research agenda for psychologists. Psychology \& Health, 26(11), 1407-1413. doi: 10.1080/08870446.2011.621703.

Benyamini, Y., \& Idler, E. L. (1999). Community studies reporting association between self-rated health and mortality: additional studies, 1995 to 1998. Research on Aging, 21(3), 392-401.

Böhm, K., Tesch-Römer, C., \& Ziese, T. (2009). Gesundheit und Krankheit im Alter [Beiträge zur Gesundheitsberichterstattung des Bundes]. Berlin: Robert Koch-Institut.

Bullinger, M., \& Kirchberger, I. (1998). Der SF-36-Fragebogen zum Gesundheitszustand. Handanweisung. Göttingen, Germany: Hogrefe.

Chen, H., Chen, P., \& Kasen, S. (2007). Cohort differences in self-rated health: evidence from a threedecade, community-based, longitudinal study of women. American Journal of Epidemiology, 166(4), 439-446.

Chipperfield, J. G. (1993). Incongruence between health perceptions and health problems: Implications for survival among seniors. Journal of Aging and Health, 5(4), 475-496.

Crimmins, E. M., \& Beltrán-Sánchez, H. (2011). Mortality and morbidity trends: is there compression of morbidity? The Journals of Gerontology, Series B: Psychological Sciences and Social Sciences, 66(1), $75-86$.

Dening, T. R., Chi, L.-Y., Brayne, C., Huppert, F. A., Paykel, E. S., \& O'Connor, D. W. (1998). Changes in selfrated health, disability and contact with services in a very elderly cohort: a 6-year follow-up study. Age and Ageing, 27(1), 23-33.

Fuchs, J., Busch, M., Lange, C., \& Scheidt-Nave, C. (2012). Prevalence and patterns of morbidity among adults in Germany. Bundesgesundheitsblatt - Gesundheitsforschung - Gesundheitsschutz, 55(4), $576-$ 586. 
Galenkamp, H., Huisman, M., Braam, A. W., \& Deeg, D. J. (2012). Estimates of prospective change in self-rated health in older people were biased owing to potential recalibration response shift. Journal of Clinical Epidemiology, 65(9), 978-988.

Heikkinen, E., Kauppinen, M., Rantanen, T., Leinonen, R., Lyyra, T.-M., Suutama, T., \& Heikkinen, R.-L. (2011). Cohort differences in health, functioning and physical activity in the young-old Finnish population. Aging clinical and experimental research, 23(2), 126-134.

Hoeymans, N., Feskens, E., Bos, G. van den, \& Kromhout, D. (1997). Age, time, and cohort effects on functional status and self-rated health in elderly men. American Journal of Public Health, 87(10), 16201625.

Idler, E. L. (1993). Age differences in self-assessments of health: Age changes, cohort differences, or survivorship? Journal of Gerontology, 48(6), S289-S300.

Idler, E. L., \& Benyamini, Y. (1997). Self-rated health and mortality: a review of twenty-seven community studies. Journal of Health and Social Behavior, 38(1), 21-37.

Jylhä, M. (2009). What is self-rated health and why does it predict mortality? Towards a unified conceptual model. Social Science \& Medicine, 69(3), 307-316. doi: 10.1016/j.socscimed.2009.05.013.

Jylhä, M., Guralnik, J. M., Balfour, J., \& Fried, L. P. (2001). Walking difficulty, walking speed, and age as predictors of self-rated health: the women's health and aging study. The Journals of Gerontology Series A: Biological Sciences and Medical Sciences, 56(10), M609-M617.

Kautzky-Willer, A. (2014). Gendermedizin. Bundesgesundheitsblatt - Gesundheitsforschung - Gesundheitsschutz, 57(9), 1022-1030.

Knecht, M. K., Bauer, G. F., Gutzwiller, F., \& Hämmig, O. (2011). Persistent work-life conflict and health satisfaction - a representative longitudinal study in Switzerland. BMC Public Health, 11(1), 271. doi:10.1186/1471-2458-11-271.

Kroll, L. E., \& Ziese, T. (2009). Kompression oder Expansion der Morbidität? In: K. Böhm, C. Tesch-Römer \& T. Ziese (Hrsg.) Gesundheit und Krankheit im Alter [Beiträge zur Gesundheitsberichterstattung des Bundes] (S. 105-112). Berlin: Robert Koch-Institut.

Leinonen, R., Heikkinen, E., \& Jylhä, M. (2001). Predictors of decline in self-assessments of health among older people - a 5-year longitudinal study. Social Science \& Medicine, 52(9), 1329-1341. doi: 10.1016/ S0277-9536(00)00249-5.

Lynch, S. M. (2003). Cohort and life-course patterns in the relationship between education and health: A hierarchical approach. Demography, 40(2), 309-331.

McArdle, J. J. (2001). A latent difference score approach to longitudinal dynamic structural analysis. In: R. Cudeck, S. du Toit \& D. Sorbom (Hrsg.) Structural equation modeling: Present and future. A Festschrift in honor of Karl Jöreskog (S. 341-380). Chicago: Scientific Software International.

McArdle, J. J., \& Hamagami, F. (2001). Latent difference score structural models for linear dynamic analyses with incomplete longitudinal data. In: L. M. Collins \& A. G. Sayer (Hrsg.) New methods for the analysis of change (S. 139-175). Washington, DC: American Psychological Association.

Menning, S., \& Hoffmann, E. (2009). Funktionale Gesundheit und Pflegebedürftigkeit. In: K. Böhm, C. Tesch-Römer \& T. Ziese (Hrsg.) Gesundheit und Krankheit im Alter [Beiträge zur Gesundheitsberichterstattung des Bundes] (S. 62-78). Berlin: Robert Koch-Institut.

Nejati, V., Shirinbayan, P., Akbari Kamrani, A., Foroughan, M., Taheri, P., \& Sheikhvatan, M. (2008). Quality of life in elderly people in Kashan, Iran. Middle East Journal of Age and Ageing, 5(2), 21-25.

Pietschmann, P., Rauner, M., Sipos, W., \& Kerschan-Schindl, K. (2009). Osteoporosis: an age-related and gender-specific disease - a mini-review. Gerontology, 55(1), 3-12. 
Prentice, A. M. (2006). The emerging epidemic of obesity in developing countries. International Journal of Epidemiology, 35(1), 93-99. doi: 10.1093/ije/dyi272.

Schöllgen, I., Huxhold, O., \& Tesch-Römer, C. (2010). Socioeconomic status and health in the second half of life: findings from the German Ageing Survey. European Journal of Ageing, 7(1), 17-28.

Schöllgen, I., Morack, J., Infurna, F. J., Ram, N., \& Gerstorf, D. (2016). Health sensitivity: Age differences in the within-person coupling of individuals' physical health and well-being. Developmental Psychology, 52(11), 1944-1953. doi: 10.1037/dev0000171.

Sprangers, M. A., \& Schwartz, C. E. (1999). Integrating response shift into health-related quality of life research: a theoretical model. Social Science \& Medicine, 48(11), 1507-1515. doi: 10.1016/S02779536(99)00045-3.

Spuling, S. M., Wolff, J. K., \& Wurm, S. (2017a). Response shift in self-rated health after serious health events in old age. Social Science \& Medicine, 192, 85-93. doi: 10.1016/j.socscimed.2017.09.026.

Spuling, S. M., Wurm, S., Tesch-Römer, C., \& Huxhold, O. (2015). Changing predictors of self-rated health: Disentangling age and cohort effects. Psychology and Aging, 30(2), 462-474. doi: 10.1037/a0039111.

Spuling, S. M., Wurm, S., Wolff, J. K., \& Wünsche, J. (2017a). Heißt krank zu sein sich auch krank zu fühlen? Subjektive Gesundheit und ihr Zusammenhang mit anderen Gesundheitsdimensionen. In: K. Mahne, J. K. Wolff, J. Simonson \& C. Tesch-Römer (Hrsg.) Altern im Wandel. Zwei Jahrzehnte Deutscher Alterssurvey (DEAS) (S. 157-170). Wiesbaden: Springer VS.

Spuling, S. M., Ziegelmann, J. P., \& Wünsche, J. (2017b). Was tun wir für unsere Gesundheit? Gesundheitsverhalten in der zweiten Lebenshälfte. In: K. Mahne, J. K. Wolff, J. Simonson \& C. Tesch-Römer (Hrsg.) Altern im Wandel. Zwei Jahrzehnte Deutscher Alterssurvey (DEAS) (S. 139-156): Wiesbaden: Springer VS.

Trachte, F., Sperlich, S., \& Geyer, S. (2015). Kompression oder Expansion der Morbidität? Zeitschrift für Gerontologie und Geriatrie, 48(3), 255-262. doi: 10.1007/s00391-014-0644-7.

Wettstein, M., Schilling, O. K., \& Wahl, H.-W. (2016). "Still feeling healthy after all these years": The paradox of subjective stability versus objective decline in very old adults' health and functioning across five years. Psychology and Aging, 31(8), 815-830. doi: 10.1037/pag0000137.

World Health Organization (2002). Towards a common language for functioning, disability and health The International Classification of Functioning, Disability and Health. Genf: WHO.

Wills, T. A. (1992). The Role of Similarity in Coping Through Downward Comparison. In: L. Montada, S.-H. Filipp \& M. J. Lerner (Hrsg.) Life crises and experiences of loss in adulthood (S. 196-212). Hillsdale, NJ, US: Lawrence Erlbaum Associates.

Wolff, J. K., Nowossadeck, S., \& Spuling, S. M. (2017). Altern nachfolgende Kohorten gesünder? Selbstberichtete Erkrankungen und funktionale Gesundheit im Kohortenvergleich. In: K. Mahne, J. K. Wolff, J. Simonson \& C. Tesch-Römer (Hrsg.) Altern im Wandel. Zwei Jahrzehnte Deutscher Alterssurvey (DEAS) (S. 125-138). Wiesbaden: Springer VS.

Wurm, S., Lampert, T., \& Menning, S. (2009). Subjektive Gesundheit. In: K. Böhm, C. Tesch-Römer \& T. Ziese (Hrsg.) Gesundheit und Krankheit im Alter [Beiträge zur Gesundheitsberichterstattung des Bundes] (S. 79-91). Berlin: Robert Koch-Institut.

Zajacova, A., Huzurbazar, S., \& Todd, M. (2017). Gender and the structure of self-rated health across the adult life span. Social Science \& Medicine, 187, 58-66. doi: 10.1016/j.socscimed.2017.06.019. 
Open Access Dieses Kapitel wird unter der Creative Commons Namensnennung 4.0 International Lizenz (http:// creativecommons.org/licenses/by/4.0/deed.de) veröffentlicht, welche die Nutzung, Vervielfältigung, Bearbeitung, Verbreitung und Wiedergabe in jeglichem Medium und Format erlaubt, sofern Sie den/die ursprünglichen Autor(en) und die Quelle ordnungsgemäß nennen, einen Link zur Creative Commons Lizenz beifügen und angeben, ob Änderungen vorgenommen wurden.

Die in diesem Kapitel enthaltenen Bilder und sonstiges Drittmaterial unterliegen ebenfalls der genannten Creative Commons Lizenz, sofern sich aus der Abbildungslegende nichts anderes ergibt. Sofern das betreffende Material nicht unter der genannten Creative Commons Lizenz steht und die betreffende Handlung nicht nach gesetzlichen Vorschriften erlaubt ist, ist für die oben aufgeführten Weiterverwendungen des Materials die Einwilligung des jeweiligen Rechteinhabers einzuholen. 


\title{
4. Lebenszufriedenheit und depressive Symptome bei Frauen und Männern im Verlauf der zweiten Lebenshälfte
}

\author{
Markus Wettstein und Svenja M. Spuling
}

\section{Kernaussagen}

Die Lebenszufriedenheit nimmt im höheren Alter etwas ab, und das Depressionsrisiko steigt an. Diese Veränderungen fallen bei Frauen deutlicher aus als bei Männern: Um das 60. Lebensjahr sind Frauen im Durchschnitt zunächst zufriedener mit ihrem Leben als Männer; mit zunehmendem Alter setzt jedoch ein Rückgang in der Lebenszufriedenheit ein, der bei Frauen stärker ausfällt als bei Männern. Klinisch auffällige depressive Symptome kommen bei Frauen allgemein häufiger vor als bei Männern, und zudem steigt die Wahrscheinlichkeit für diese Symptome mit zunehmendem Alter bei Frauen stärker an als bei Männern.

Nachfolgende Kohorten weisen günstigere Alternsverläufe der Lebenszufriedenheit auf, dies gilt für Frauen und Männer gleichermaßen: Die Abnahme der Lebenszufriedenheit mit zunehmendem Alter ist stärker ausgeprägt in der früher geborenen Kohorte (1930-1939 Geborene) im Vergleich zu später geborenen Kohorten (1940-1949 sowie 1950-1959 Geborene).

Nachfolgende Kohorten unterscheiden sich nicht bedeutsam in ihren Alternsverläufen depressiver Symptome, auch dies gilt für Frauen und Männer gleichermaßen: Für den Alternsverlauf des Depressionsrisikos zeigt sich kein signifikanter Kohortenunterschied. Vergleicht man zudem die Verläufe über die hier untersuchten Geburtskohorten zwischen Männern und Frauen, so tritt kein statistisch bedeutsamer Unterschied auf. Der Unterschied im Alternsverlauf zwischen Frauen und Männern fällt also in allen Geburtskohorten ähnlich aus.

\subsection{Einleitung}

Glücklich zu sein ist zweifellos ein wünschenswerter Zustand, den die meisten Personen, unabhängig von ihrem Alter, anstreben. Um Frauen und Männern ein gutes und glückliches Leben im Alter zu ermöglichen, ein wichtiges Ziel der Politik, ist es wichtig zu wissen, wie es ihnen in der zweiten Lebenshälfte geht. Ebenso sollten Erkenntnisse darüber gewonnen werden, wie sehr und in welche
Richtung sich ihre Lebensqualität mit zunehmendem Alter verändert, und ob diese Veränderungen für Frauen und Männer unterschiedlich ausfallen. Möglicherweise sind in einer bestimmten Lebensphase Frauen stärker in ihrer Lebensqualität eingeschränkt als Männer (oder umgekehrt), so dass für mögliche Interventionen „Risiko-Lebensphasen“ identifiziert werden können, die unterschied- 
lich ausfallen könnten für Frauen und Männer. Schließlich stellt sich auch die Frage, inwieweit der gesellschaftliche Wandel, charakterisiert unter anderem durch technischen und medizinischen Fortschritt sowie veränderte Arbeits- und Lebensbedingungen, dazu geführt hat, dass sich Alternsverläufe der Lebensqualität über nachfolgende Geburtskohorten verändert haben. Hieran knüpft sich wiederum unmittelbar die Frage nach Geschlechterunterschieden an: Haben Frauen und Männer gleichermaßen vom gesellschaftlichen Wandel profitiert, so dass die über die Geburtskohorten gefundenen Trends - sofern vorhanden gleichermaßen auf Frauen und Männer zutreffen? Oder hat sich der gesellschaftliche Wandel unterschiedlich ausgewirkt auf die Alternsverläufe der Frauen und die der Männer?

In diesem Kapitel wird untersucht, wie es Frauen und Männern geht, wenn sie älter werden. Dafür müssen unterschiedliche Maße herangezogen werden, denn Lebensqualität und psychisches Wohlbefinden setzen sich aus verschiedenen Komponenten zusammen (Diener, Suh, Lucas, \& Smith 1999), die innerhalb einer Person unterschiedlich ausgeprägt sein können. Deshalb werden in diesem Kapitel zwei Maße herangezogen: die Lebenszufriedenheit sowie depressive Symptome. Ein weiteres Maß zur Beschreibung der subjektiven Lebensqualität, das Einsamkeitserleben, wird in Kapitel 5 Soziale Isolation und Einsamkeit in diesem Band thematisiert.

Die Lebenszufriedenheit entspricht einer globalen Bewertung der eigenen Lebensqualität („Ich bin mit meinem Leben zufrieden“; Diener et al. 1999) und kann dem „hedonischen Wohlbefinden“ (Ryan \& Deci 2001) zugeordnet werden, dessen Kernaspekte das Anstreben von Vergnügen und das Vermeiden von Schmerz sind. Dagegen sind depressive Symptome Komponenten „negativen Wohlbefindens“ („ill-being“; Ryff, Dienberg Love, Urry, Muller, Rosenkranz, Friedman, Davidson, \& Singer 2006), und sie schließen verschiedene Aspekte ein (Radloff 1977). Dazu gehören emotionale Aspekte wie das Erleben depressiver Verstimmung (z.B. Empfinden von Traurigkeit) oder der
Mangel an positiven Emotionen (etwa an Lebensfreude), aber auch weitere Aspekte wie somatische Beschwerden (z.B. Schlaflosigkeit) und interpersonelle Probleme (wie z.B. das Gefühl, von anderen nicht gemocht zu werden). Wichtig ist es, beide Komponenten zu untersuchen, denn Veränderungstrends in der Lebenszufriedenheit mit zunehmendem Alter können durchaus anders ausfallen als Veränderungstrends in depressiver Symptomatik (z. B. Wettstein, Schilling, Reidick, \& Wahl 2015).

\section{Unterscheiden sich Frauen und Männer in ihren Alternsverläufen von Lebenszufriedenheit und depressiven Symptomen?}

Wie unterscheiden sich Frauen und Männer in ihrer Lebenszufriedenheit und ihrer depressiven Symptomatik? Und verändern sich diese mit zunehmendem Alter bei Frauen und Männern in unterschiedlicher Weise? Ältere Frauen sind etwas weniger mit ihrem Leben zufrieden als ältere Männer (Pinquart \& Sörensen 2001; Wolff \& TeschRömer 2017), während Geschlechterunterschiede hinsichtlich der allgemeinen Lebenszufriedenheit in früheren Altersphasen eher gering sind. Zudem sind Frauen häufiger von depressiven Symptomen betroffen als Männer (Sutin, Terracciano, Milaneschi, An, Ferrucci, \& Zonderman 2013b; TeschRömer, Motel-Klingebiel, \& Tomasik 2008; Wolff \& Tesch-Römer 2017), und dieser Unterschied wird mit zunehmendem Alter größer. Dies könnte unter anderem daran liegt, dass Frauen im Alter stärker von gesundheitlichen Problemen betroffen sind als Männer (siehe Kapitel 3 Funktionale und subjektive Gesundheit in diesem Band), und diese gesundheitlichen Probleme sind ihrerseits Risikofaktoren für depressive Verstimmungen (z.B. Diegelmann, Schilling, \& Wahl 2016).

Was ist bereits bekannt darüber, ob und wie sich Frauen und Männer verschiedenen Alters darin unterscheiden, wie zufrieden sie mit ihrem Leben sind oder wie sehr sie von depressiven Symptomen 
betroffen sind? Gemäß des Wohlbefindensoder Zufriedenheitsparadoxons (Herschbach 2002; Staudinger 2000) unterscheiden sich Personen unterschiedlichen Alters nicht oder nur geringfügig in ihrer Lebenszufriedenheit. Das mag deshalb "paradox" erscheinen, da mit dem Alter etwa die Gesundheit im Allgemeinen nachlässt (siehe Kapitel 3 Funktionale und subjektive Gesundheit in diesem Band), und man daher erwarten könnte, dass Frauen und Männer mit zunehmendem Alter weniger zufrieden mit ihrem Leben sind. Tatsächlich bleibt aber die Lebenszufriedenheit relativ stabil beim Vergleich über verschiedene Altersgruppen hinweg (Wolff \& Tesch-Römer 2017), und sogar bei Personen im (sehr) hohen Alter verändert sich die Lebenszufriedenheit im Durchschnitt kaum (Wettstein et al. 2015). Andererseits muss jedoch einschränkend konstatiert werden, dass längsschnittliche Analysen von Lebenszufriedenheitsverläufen keine völlige Stabilität bestätigen konnten: Vielmehr tritt etwa ab dem 60. Lebensjahr im Durchschnitt - bei sehr großer Unterschiedlichkeit individueller Verläufe - ein leichter Rückgang in der Lebenszufriedenheit auf (Mroczek \& Spiro 2005; Schilling 2006), der in den letzten drei bis fünf Lebensjahren deutlicher ausfällt (Gerstorf, Ram, Mayraz, Hidajat, Lindenberger, Wagner, \& Schupp 2010). Allerdings verändern sich Lebenszufriedenheit und depressive Symptome keineswegs bei allen Frauen und Männern in gleicher Weise. Vielmehr sind die beobachteten Alternsverläufe sehr unterschiedlich von Individuum zu Individuum.

\section{Unterscheiden sich nachfolgende Geburtsko- horten in ihren Alternsverläufen von Lebens- zufriedenheit und depressiven Symptomen, und fallen diese Kohortentrends für Frauen und Männer unterschiedlich aus?}

Unsere Gesellschaft befindet sich im fortwährenden Wandel. Faktoren wie technischer und medizinischer Fortschritt, ein Wandel in Lebensstil und Gesundheitsverhalten sowie veränderte Ar- beits- und Lebensbedingungen könnten auch eine Veränderung der Lebenszufriedenheit und der depressiven Symptomatik sowie ihrer Alternsverläufe über nachfolgende Geburtsjahrgänge mit sich bringen.

Allerdings fallen die Ergebnisse bisheriger Kohortenvergleiche der Lebenszufriedenheit sehr unterschiedlich aus: So gibt es Befunde, dass später geborene Kohorten im höheren Alter eine höhere Lebenszufriedenheit berichten sowie geringere Einbußen der Lebenszufriedenheit mit zunehmendem Alter aufweisen als früher geborene Kohorten (Hülür, Ram, \& Gerstorf 2015; Sutin, Terracciano, Milaneschi, An, Ferrucci, \& Zonderman 2013a). Es gibt aber auch gegenteilige Befundmuster (Schilling 2005).

Ähnlich uneinheitlich verhält es sich mit Kohorten-Befunden zu depressiven Symptomen: In früheren Auswertungen des Deutschen Alterssurveys (DEAS) hat sich gezeigt, dass zumindest für ältere Frauen und Männer (66-83 Jahre) bei nachfolgenden Kohorten die depressiven Symptome über den Zeitraum von 2002 bis 2014 zurückgegangen sind (Wolff \& Tesch-Römer 2017). Dagegen haben Murphy, Laird, Monson, Sobol, und Leighton (2000) eine relativ stabile Prävalenz klinischer Depression bei 18-Jährigen und Älteren im Zeitraum von 1952 bis 1992 beobachtet. In anderen Nationen, etwa China und Belgien, wurde ein Anstieg depressiver Symptome über die Kohorten allgemein (Brault, Meuleman, \& Bracke 2012), oder zumindest bei älteren Erwachsenen (Shao, Li, Zhang, Zhang, Zhang, \& Qi 2013), festgestellt. Was Kohortenunterschiede in Alternsverläufen depressiver Symptome betrifft, berichten einige Studien günstigere Alternsverläufe über nachfolgende Kohorten hinweg (Kasen, Cohen, Chen, \& Castille 2003), während andere Studien weniger günstige Alternsverläufe bei nachfolgenden Kohorten beobachtet haben (Yang 2007).

Aufgrund dieser uneinheitlichen Befundlage sind unterschiedliche Annahmen denkbar, wie sich bei den Teilnehmerinnen und Teilnehmern des Deutschen Alterssurveys Alternsverläufe der Lebenszufriedenheit und der depressiven Symp- 
tome über die Kohorten gewandelt haben. Möglicherweise haben sich nicht alle Lebensbedingungen verbessert, zum Beispiel stehen dem medizinischen Fortschritt auf der einen Seite berufliche Arbeitsanforderungen auf der anderen Seite gegenüber, die im Wandel der Zeit vielleicht eher belastender geworden sind. Daher sind von einem positiven oder negativen bis hin zu einem ausbleibenden Kohorteneffekt alle Szenarien denkbar.

Die Frage, ob sich der gesellschaftliche Wandel unterschiedlich auswirkt auf die Alternsverläufe der Frauen und Männer, also ob Kohortentrends für beide Geschlechter verschieden ausfallen, wurde unseres Wissens noch nicht systematisch untersucht. Murphy et al. (2000) beobachteten einen geschlechtsspezifischen Kohorteneffekt, nämlich ein höheres Depressionsrisiko bei Frauen in einer im Jahr 1992 gezogenen Stichprobe im Vergleich zu einer im Jahr 1970 gezogenen Stichprobe, aber kein höheres Risiko bei Männern 1992 im Vergleich zu 1970. Jedoch untersuchten die Autoren nicht den Alternsverlauf depressiver Symptome in diesen beiden Stichproben. Einen stärkeren Anstieg depressiver Symptome bei Frauen als bei Männern über verschiedene, 24 Jahre auseinander liegende Stichproben chinesischer Erwachsener hinweg berichten zudem Shao et al. (2013), jedoch beobachteten auch sie diese Stichproben nicht längsschnittlich und können daher keine Aussage über Geschlechterunterschiede in Alternsverläufen treffen. Kasen et al. (2003) verglichen den Alternsverlauf depressiver Symptome - allerdings nur bei Frauen, und nur über das mittlere Erwachsenenalter - für zwei Kohorten, Babyboomer und Preboomer, und fanden einen Rückgang der depressiven Symptome im Alternsverlauf bei der Babyboomer-Kohorte, jedoch einen Anstieg in der Preboomer-Kohorte.

Denkbar ist in Bezug auf Geschlechterunterschiede in Kohortentrends, dass es zu einer Angleichung der Alternsverläufe von Frauen und Männern über nachfolgende Geburtskohorten gekommen ist, da die Lebensbedingungen von Frauen und Männern teilweise ähnlicher geworden sind: In später geborenen Kohorten sind mehr
Frauen berufstätig als in früher geborenen, so dass der Geschlechterunterschied hinsichtlich Berufstätigkeit kleiner geworden ist. Ähnliches gilt für die Bildung, die einen Schutzfaktor darstellt in Bezug auf Lebenszufriedenheit und depressive Symptome (Wolff \& Tesch-Römer 2017) und hinsichtlich derer sich Frauen und Männer heute weniger unterscheiden als noch vor einigen Jahrzehnten. Andererseits könnten Unterschiede zwischen Frauen und Männern hinsichtlich ihrer Gesundheit stabil geblieben sein über verschiedene Geburtskohorten hinweg (siehe Kapitel 3 Funktionale und subjektive Gesundheit in diesem Band). Zwar hat sich das Gesundheitsverhalten von Frauen und Männern in unterschiedlicher Weise verändert (Spuling, Ziegelmann, \& Wünsche 2017), diese Geschlechterunterschiede in der Gesundheit werden aber teilweise durch biologische Faktoren bedingt, auf die der gesellschaftliche Wandel keinen Einfluss hat. Insofern ist eine Angleichung der Alternsverläufe von Frauen und Männern hinsichtlich ihrer Lebenszufriedenheit und ihrer depressiven Symptomatik über nachfolgende Kohorten ebenso denkbar wie eine kohortenübergreifende Stabilität der Geschlechterunterschiede in den Alternsverläufen.

\section{Fragestellungen}

Folgende Forschungsfragen werden in diesem Kapitel untersucht:

1) Unterscheiden sich Frauen und Männer in ihren Alternsverläufen von Lebenszufriedenheit und depressiven Symptomen?

2) Wie wandeln sich die Alternsverläufe der Lebenszufriedenheit und der depressiven Symptome über verschiedene Geburtskohorten bei Frauen und Männern? 


\subsection{Methoden}

\section{Stichprobe}

Verwendet wurden für die nachfolgenden Analysen die Daten des Deutschen Alterssurveys (DEAS). Die Untersuchungsstichprobe bestand aus 20038 Studienteilnehmenden, die seit $1996 \mathrm{zu}$ mindestens einem der Erhebungszeitpunkte an der Studie teilgenommen haben. Details zur Stichprobe sowie zu Methoden sind in Kapitel 2 Design, Inhalte und Methodik des Deutschen Alterssurveys in diesem Band beschrieben.

\section{Indikatoren}

Lebenszufriedenheit. Zur Erfassung der Lebenszufriedenheit wird die etablierte „Satisfaction with Life Scale“ (Diener, Emmons, Larsen, \& Griffin 1985) in deutscher Fassung (Glaesmer, Grande, Braehler, \& Roth 2011) verwendet. Die Skala wird im schriftlichen Fragebogen vorgegeben und besteht aus fünf Items (z. B. „In den meisten Dingen ist mein Leben nahezu ideal“; „Ich bin mit meinem Leben zufrieden"), welche von den Befragten auf einer Antwortskala mit einem Range von "trifft gar nicht zu“ (1) bis „trifft genau zu“ (5) beantwortet werden. Die Antworten jeder befragten Person werden zu einem Wert zusammengefasst, indem sie gemittelt werden. Dabei bedeuten höhere Werte eine höhere Lebenszufriedenheit (1: sehr unzufrieden, 2: unzufrieden, 3: weder zufrieden noch unzufrieden; 4: zufrieden, 5: sehr zufrieden).

Depressive Symptome. Anders als Lebenszufriedenheit wurden depressive Symptome erstmals 2002 (und nicht 1996) erhoben. Daher liegen den folgenden Auswertungen zu den depressiven Symptomen die Angaben aller Personen (15223) zugrunde, die ab 2002 am Deutschen Alterssurvey teilgenommen haben. Die „Center for Epidemiological Studies Depression Scale“ (CES-D; Radloff 1977) beziehungsweise ihre deutschsprachige Adaptation, die Kurzversion der Allgemeinen Depressionsskala (ADS-K; Hautzinger \& Bailer 1993), wird zur Erfassung depressiver Symptome im mündlichen Interview eingesetzt. Die Skala besteht aus 15 Items (z. B. „In der letzten Woche dachte ich, mein Leben ist ein einziger Fehlschlag“; „In der letzten Woche war alles anstrengend für mich"), die von den Befragten jeweils auf einer Häufigkeitsskala von „selten/überhaupt nicht (weniger als 1 Tag lang)“ bis „meistens, die ganze Zeit (5 bis 7 Tage lang)“ beantwortet werden. Hier wurde pro Person ein Summenwert über alle Items gebildet, der zwischen 0 und 45 liegen konnte. Erreicht oder übersteigt dieser Summenwert den Wert von 18, weist dies auf klinisch auffällige depressive Symptome (beziehungsweise eine ernsthafte depressive Störung) hin (Lehr, Hillert, Schmitz, \& Sosnowsky 2008). Um zu untersuchen, wie sich das Risiko, diese klinisch auffälligen Symptome zu entwickeln, über die zweite Lebenshälfte verändert, wird im Folgenden zwischen Personen, die keine klinisch auffälligen Symptome haben (also Werte unter 18 aufweisen), und denjenigen, deren depressive Symptome klinisch auffällig sind (also einen Wert von mindestens 18 haben), unterschieden.

Gruppierungsvariable: Geburtskohorte. Die Geburtsjahrgänge 1930-1939, 1940-1949 und 19501959 wurden jeweils zu einer Kohorte zusammengefasst.

Kontrollvariable: Region. Als Kontrollvariable wurde die Region zum jeweiligen Erstbefragungszeitpunkt (West-/Ostdeutschland) in allen Analysen berücksichtigt.

\section{Statistische Analyseverfahren}

Zur Untersuchung der Alternsverläufe wurden für die Lebenszufriedenheit längsschnittliche Mehrebenen-Regressionsmodelle sowie für depressive Symptome logistische Random-Effects-Panelregressionsmodelle berechnet. Auf Grundlage dieser Methoden wird für jede Person ein individueller Alternsverlauf geschätzt. Die Schätzmethode beider Verfahren berücksichtigt selektiven Stichpro- 
benausfall, indem sie auch Studienteilnehmerinnen und -teilnehmer in die Schätzung der Verläufe einbezieht, die nur einmal am Deutschen Alterssurvey teilgenommen haben. Die beiden Analysemethoden lassen außerdem zu, die Effekte von
Prädiktoren der Alternsverläufe (hier: Geschlecht, Kohorte sowie die Interaktion von beiden) $\mathrm{zu}$ schätzen. Das Signifikanzniveau für alle inferenzstatistischen Tests wurde auf $\mathrm{p}<0,05$ festgelegt.

chen Frauen und Männer das 90. Lebensjahr, so ist die durchschnittliche Lebenszufriedenheit der Männer nur um 0,1 Punkte höher als die der Frauen.

Ebenso ist das durchschnittliche Ausmaß, in dem die Lebenszufriedenheit ab dem höheren Erwachsenenalter absinkt, gering: Bei Frauen beträgt die geschätzte mittlere Differenz zwischen ihrer „maximalen“ Lebenszufriedenheit (um das 70. Lebensjahr) und ihrer durchschnittlichen Lebenszufriedenheit, wenn sie 90 Jahre alt sind, 0,12 Punkte. Bei Männern ist diese mittlere Differenz (bei ihnen zwischen dem 80 . und dem 90. Lebensjahr) noch geringer und beträgt 0,03 Punkte. Zudem ist über den Alternsverlauf auch im sehr hohen Alter, mit 90 Jahren etwa, die durchschnittliche Lebenszufriedenheit der Frauen und Männer nahe am Wert von 4 (was auf der von 1 bis 5 reichenden Skala „zufrieden“ bedeutet) und somit deutlich über dem theoretischen Mittelwert der Skala: Die meisten Frauen und Männer sind in der zweiten Lebenshälfte mit ihrem Leben zufrieden, und sie bleiben es auch im sehr hohen Alter.

Die Rolle der Kohorten für Alternsverläufe der Lebenszufriedenheit: Günstigere Alternsverläufe der Lebenszufriedenheit für Frauen und Männer der später geborenen Kohorten.

Aus den Verlaufsschätzungen ergibt sich ein positiver Kohortentrend: Der im höheren Alter einsetzende durchschnittliche Rückgang der Lebenszufriedenheit fällt in den nachfolgenden Kohorten schwächer aus (vgl. Abbildung 4-2). Für die später geborene Kohorte (1950-1959) legt der geschätzte Alternsverlauf sogar nahe, dass diese im hohen Al- 
Abbildung 4-1 Lebenszufriedenheit bei Frauen und Männern im Alternsverlauf

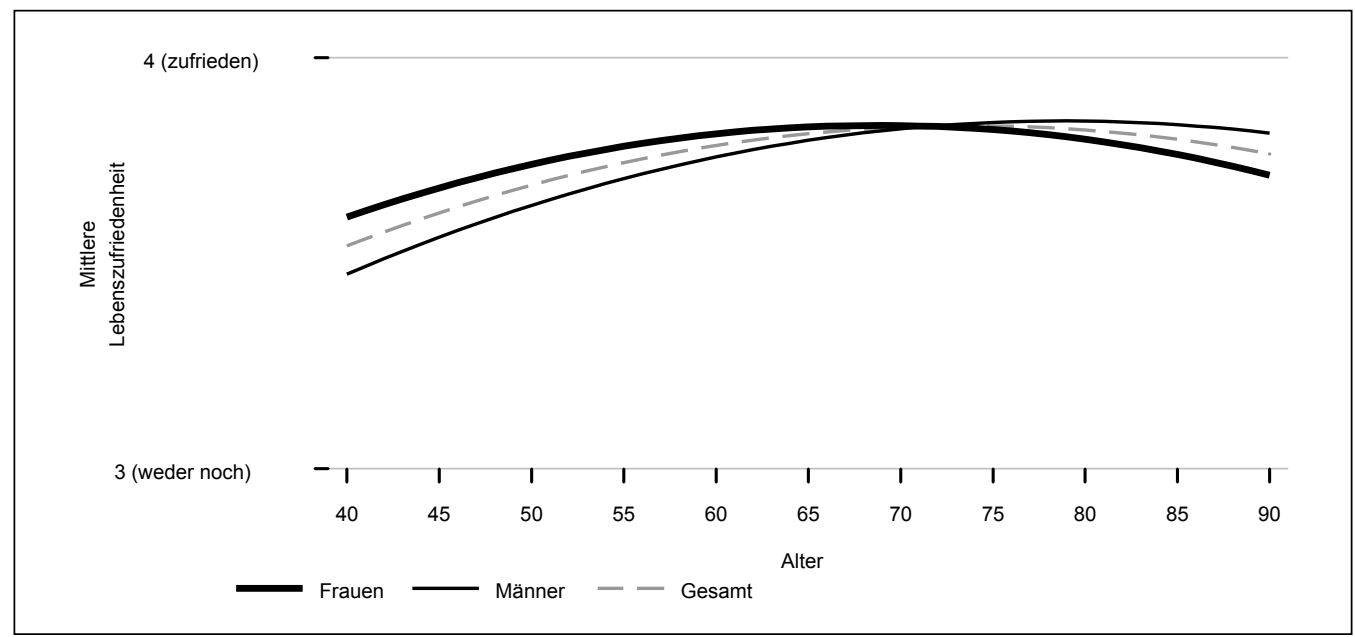

Quelle: DEAS 1996-2017; Schätzungen basieren auf $\mathrm{n}=16528$ Studienteilnehmenden mit insgesamt $\mathrm{n}=31519$ Beobachtungen; kontrolliert für Region. Die Lebenszufriedenheitsskala reicht von 1 („sehr unzufrieden“) bis 5 („sehr zufrieden“), in der Abbildung wird nur der Bereich von 3 („weder zufrieden noch unzufrieden“) bis 4 („zufrieden“) dargestellt.

Frauen sind im Durchschnitt zunächst etwas zufriedener mit ihrem Leben als Männer, allerdings nimmt die Lebenszufriedenheit bei ihnen mit zunehmendem Alter stärker ab als bei Männern. Zudem tritt dieser Rückgang bei Frauen schon früher ein als bei Männern, so dass ihre Zufriedenheitswerte im höheren und hohen Alter etwas unter denen der Männer liegen.

\section{Abbildung 4-2 Lebenszufriedenheit nach Kohorten im Alternsverlauf}

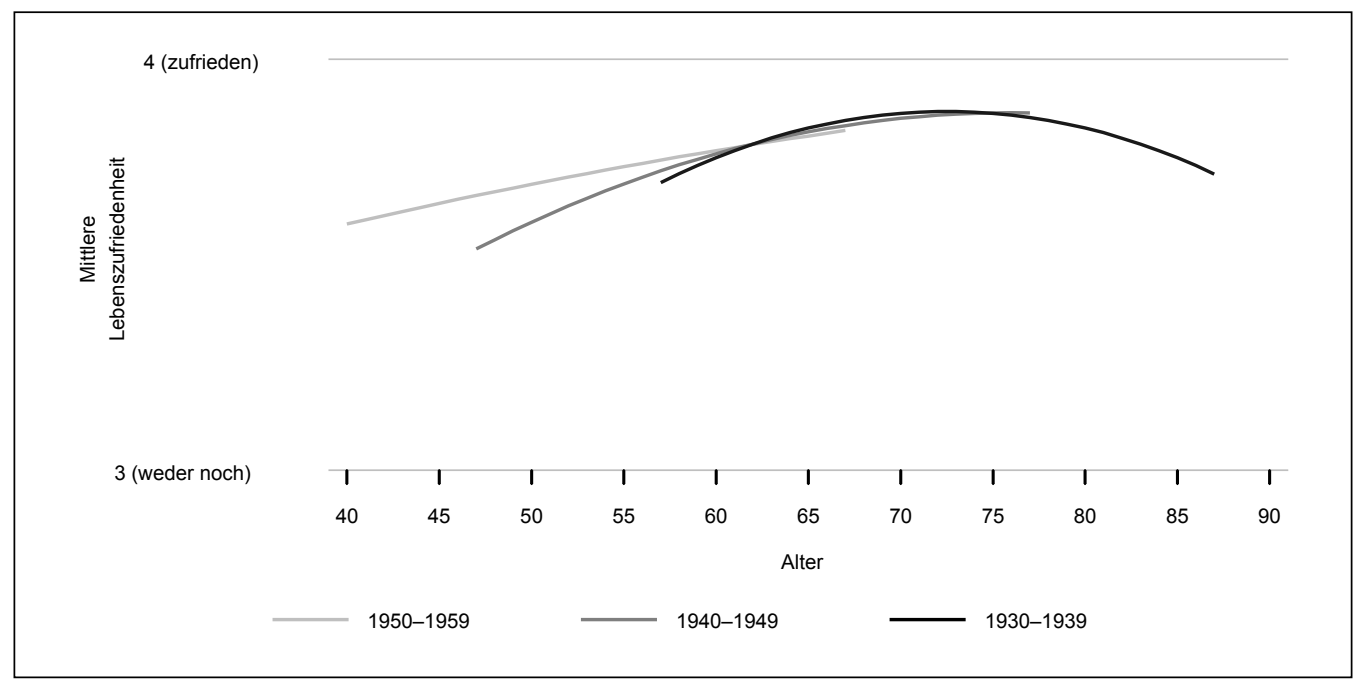

Quelle: DEAS 1996-2017; Schätzungen basieren auf $n=11655$ Studienteilnehmenden mit insgesamt $n=23802$ Beobachtungen; kontrolliert für Region und Geschlecht. Die Lebenszufriedenheitsskala reicht von 1 („sehr unzufrieden“) bis 5 („,sehr zufrieden“), in der Abbildung wird nur der Bereich von 3 („weder zufrieden noch unzufrieden“) bis 4 („zufrieden“) dargestellt.

Der geschätzte Alternsverlauf der Lebenszufriedenheit ist für die später geborenen Kohorten günstiger als für die früher geborene Kohorte. 
Abbildung 4-3 Lebenszufriedenheit bei Frauen und Männern nach Kohorten im Alternsverlauf

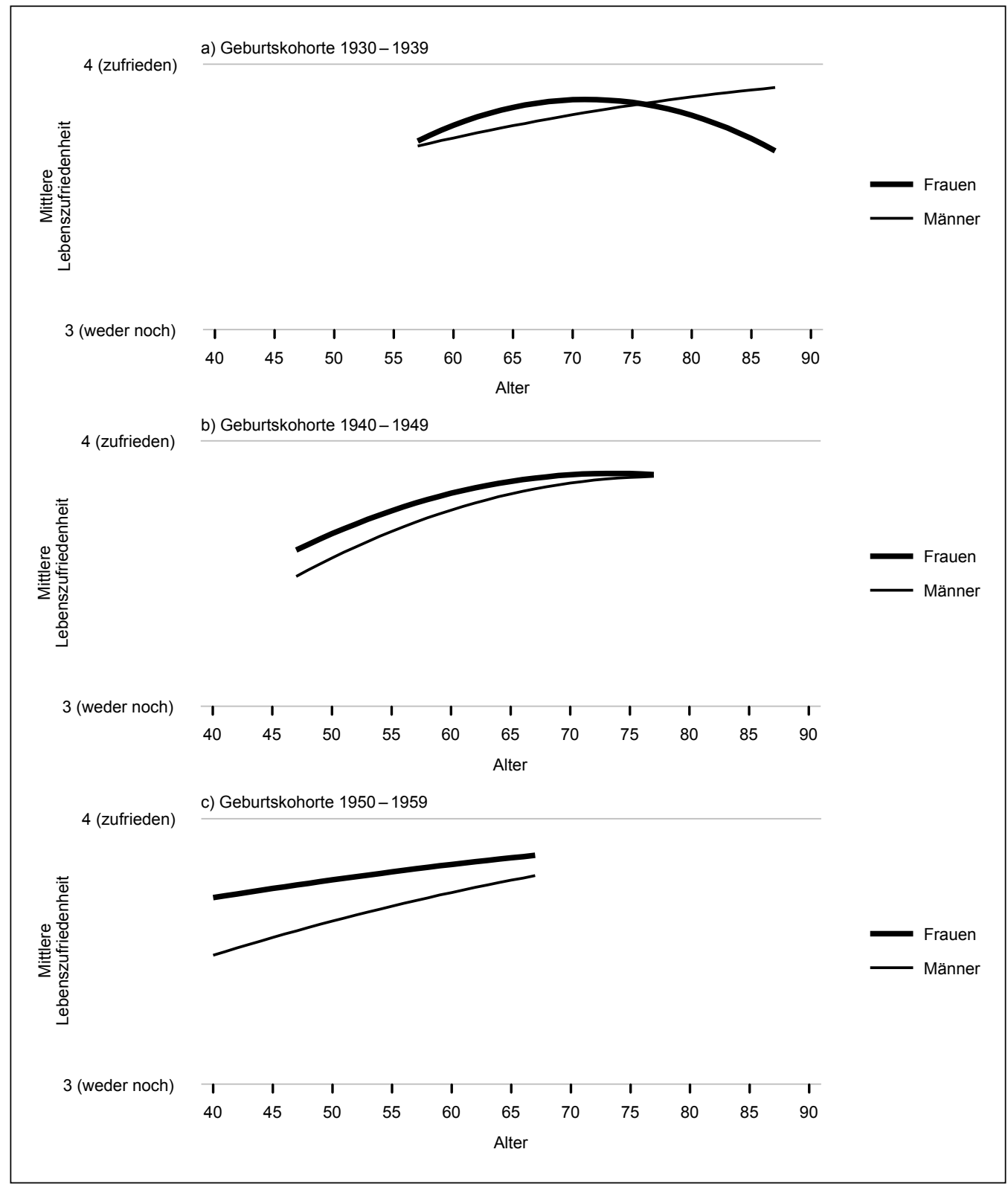

Quelle: DEAS 1996-2017; Schätzungen basieren auf $n=11655$ Studienteilnehmenden mit insgesamt $n=23802$ Beobachtungen; kontrolliert für Region. Die Lebenszufriedenheitsskala reicht von 1 („sehr unzufrieden“) bis 5 („sehr zufrieden“), in der Abbildung wird nur der Bereich von 3 („weder zufrieden noch unzufrieden“) bis 4 („zufrieden“) dargestellt.

Der geschätzte Alternsverlauf der Lebenszufriedenheit ist für die später geborenen Kohorten günstiger als für die früher geborene Kohorte. Dies gilt gleichermaßen für Frauen und Männer, da die Verlaufsunterschiede zwischen Frauen und Männern sich nicht signifikant über die nachfolgenden Kohorten verändert haben. 
ter - das dieser Geburtsjahrgang aktuell noch nicht erreicht hat - voraussichtlich durch eine stabil bleibende oder sogar leicht ansteigende Lebenszufriedenheit charakterisiert ist, während bei den früher geborenen Kohorten (1930-1939 sowie 1940-1949) ein Rückgang der Lebenszufriedenheit im hohen Alter (Kohorte 1930-1939: ca. ab dem 75. Lebensjahr; Kohorte 1940-1949: ca. ab dem 80. Lebensjahr) auftritt. Dieser positive Kohortentrend gilt gleichermaßen für Frauen und Männer, die sich hierin nicht statistisch bedeutsam unterscheiden (Abbildung 4-3).

\subsubsection{Depressive Symptome im Verlauf der zweiten Lebenshälfte}

Alternsverlauf der depressiven Symptome bei Frauen und Männern: Frauen sind häufiger von klinisch auffälligen depressiven Symptomen betroffen, und ihre Wahrscheinlichkeit für diese Symptome steigt mit dem Alter stärker an als die der Männer.

Vom 40. Lebensjahr an sinkt das Risiko für klinisch auffällige depressive Symptome zunächst, und zwar sowohl bei Frauen als auch bei Männern. Im Übergang vom höheren ins sehr hohe Alter steigt ihr durchschnittliches Risiko dagegen an.

Neben dieser grundsätzlich ähnlichen Verlaufsform bei Frauen und Männern gibt es jedoch auch Unterschiede: Über den Alternsverlauf betrachtet, haben im Alter von ca. 64 Jahren (mittleres Alter der Stichprobe) Frauen ein höheres Risiko, an klinisch auffälligen depressiven Symptomen zu leiden. Darüber hinaus ist der im Alternsverlauf auftretende Anstieg der Wahrscheinlichkeit, im hohen oder sehr hohen Alter an klinisch auffälligen Symptomen zu leiden, bei Frauen stärker als bei Männern, so dass die Diskrepanz zwischen Frauen und Männern mit steigendem Alter zu-

Abbildung 4-4 Depressionsrisiko bei Frauen und Männern im Alternsverlauf

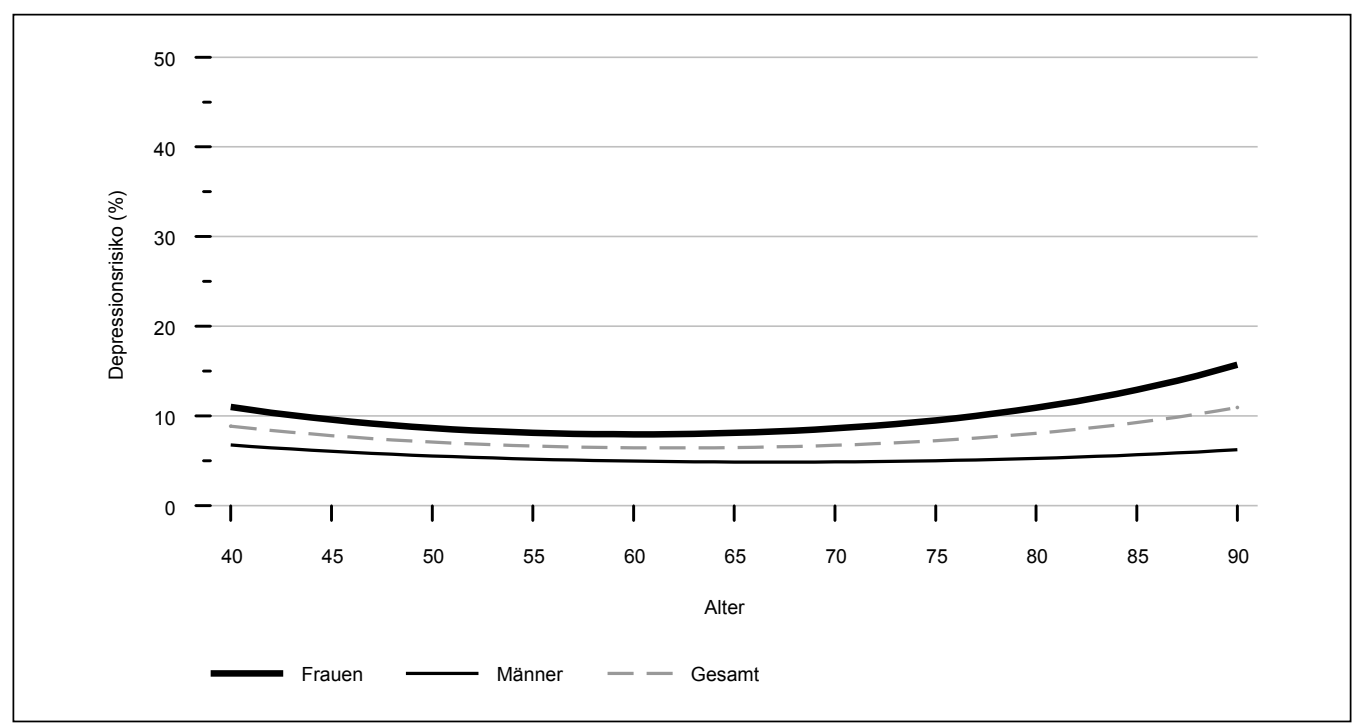

Quelle: DEAS 2002-2017; Schätzungen basieren auf $\mathrm{n}=14906$ Studienteilnehmenden mit insgesamt $\mathrm{n}=28554$ Beobachtungen; kontrolliert für Region. Der Wertebereich des Depressionsrisikos reicht von null Prozent bis 1oo Prozent, in der Abbildung wird nur der Bereich null Prozent bis 50 Prozent gezeigt.

Frauen sind, wenn sie das Alter von 64 Jahren erreichen, von einem höheren Depressionsrisiko betroffen als Männer, zudem steigt ihr Depressionsrisiko mit zunehmendem Alter stärker an als das der Männer. 
nimmt (Abbildung 4-4). Dies führt dazu, dass spät im Alternsverlauf, im Alter von etwa 90 Jahren, die geschätzte Wahrscheinlichkeit für eine klinisch auffällige Depression bei Frauen knapp 16 Prozent beträgt, während sie bei Männern mit ungefähr sechs Prozent deutlich geringer ist. Bei Frauen steigt das durchschnittliche Depressionsrisiko zudem früher im Alternsverlauf an, bereits ungefähr ab dem 60. Lebensjahr, bei Männern ist dieser Anstieg dagegen erst ab dem ca. 70. Lebensjahr zu beobachten.

Die Rolle der Kohorten für Alternsverläufe klinisch auffälliger depressiver Symptome: Weder bei Frauen noch bei Männern unterscheiden sich die Verläufe zwischen den Geburtsjahrgängen.
Anders als für die Lebenszufriedenheit unterscheiden sich die drei ausgewählten Geburtskohorten nicht hinsichtlich ihres Depressionsrisikos mit ca. 64 Jahren und hinsichtlich ihrer durchschnittlichen Alternsveränderung im Depressionsrisiko. Dieses scheint also über die untersuchten nachfolgenden Kohorten hinweg relativ stabil geblieben zu sein (Abbildung 4-5). Da es auch keine signifikante Interaktion von Kohortenzugehörigkeit und Geschlecht gibt, kann zudem gefolgert werden, dass für Frauen und Männer gleichermaßen der Alternsverlauf des Depressionsrisikos über die Kohorten, und damit auch die Diskrepanz in den Alternsverläufen zwischen Frauen und Männern, stabil geblieben ist (Abbildung 4-6).

\section{Abbildung 4-5 Depressionsrisiko nach Kohorten im Alternsverlauf}

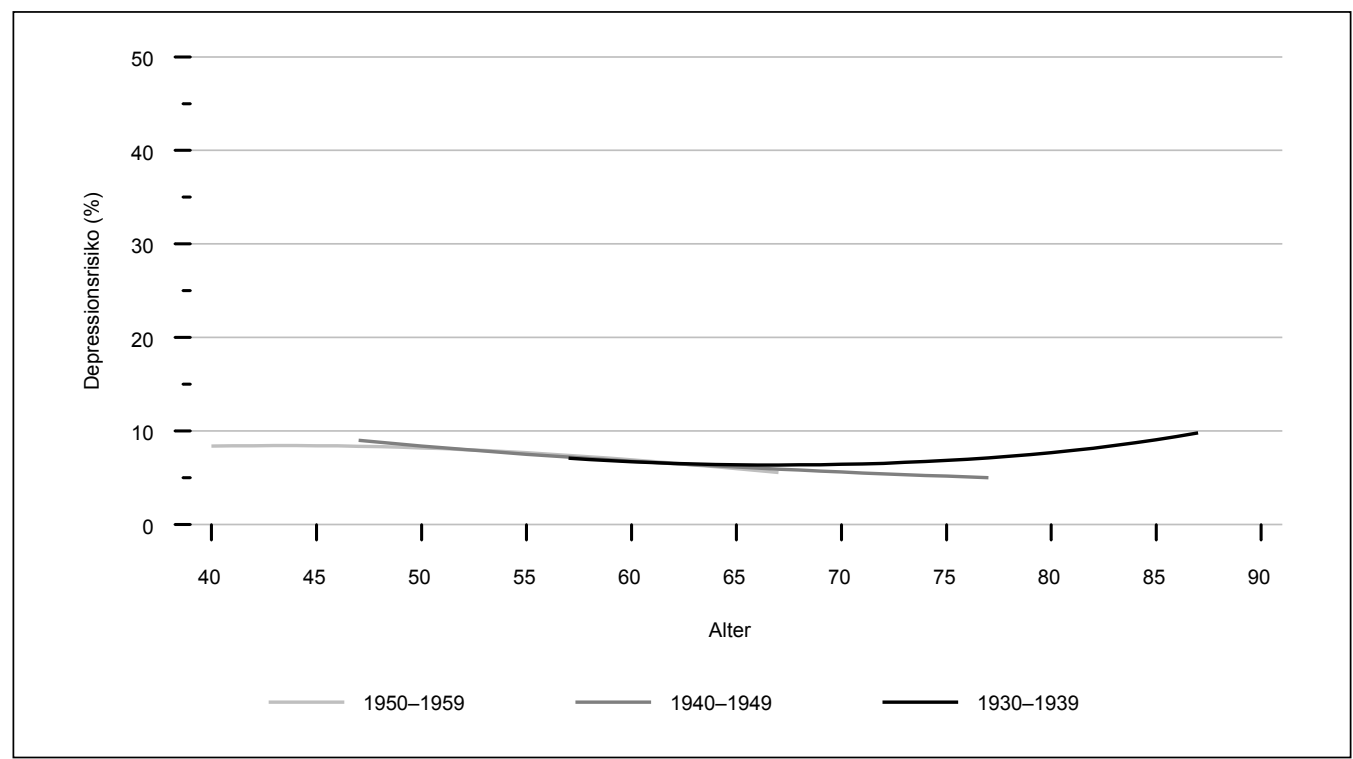

Quelle: DEAS 2002-2017; Schätzungen basieren auf $n=10504$ Studienteilnehmenden mit insgesamt $n=16677$ Beobachtungen; kontrolliert für Region und Geschlecht. Der Wertebereich des Depressionsrisikos reicht von null Prozent bis 100 Prozent, in der Abbildung wird nur der Bereich null Prozent bis 50 Prozent gezeigt.

Die Kohorten unterscheiden sich nicht signifikant im Alternsverlauf des Depressionsrisikos. 
Abbildung 4-6 Depressionsrisiko bei Frauen und Männern nach Kohorten im Alternsverlauf

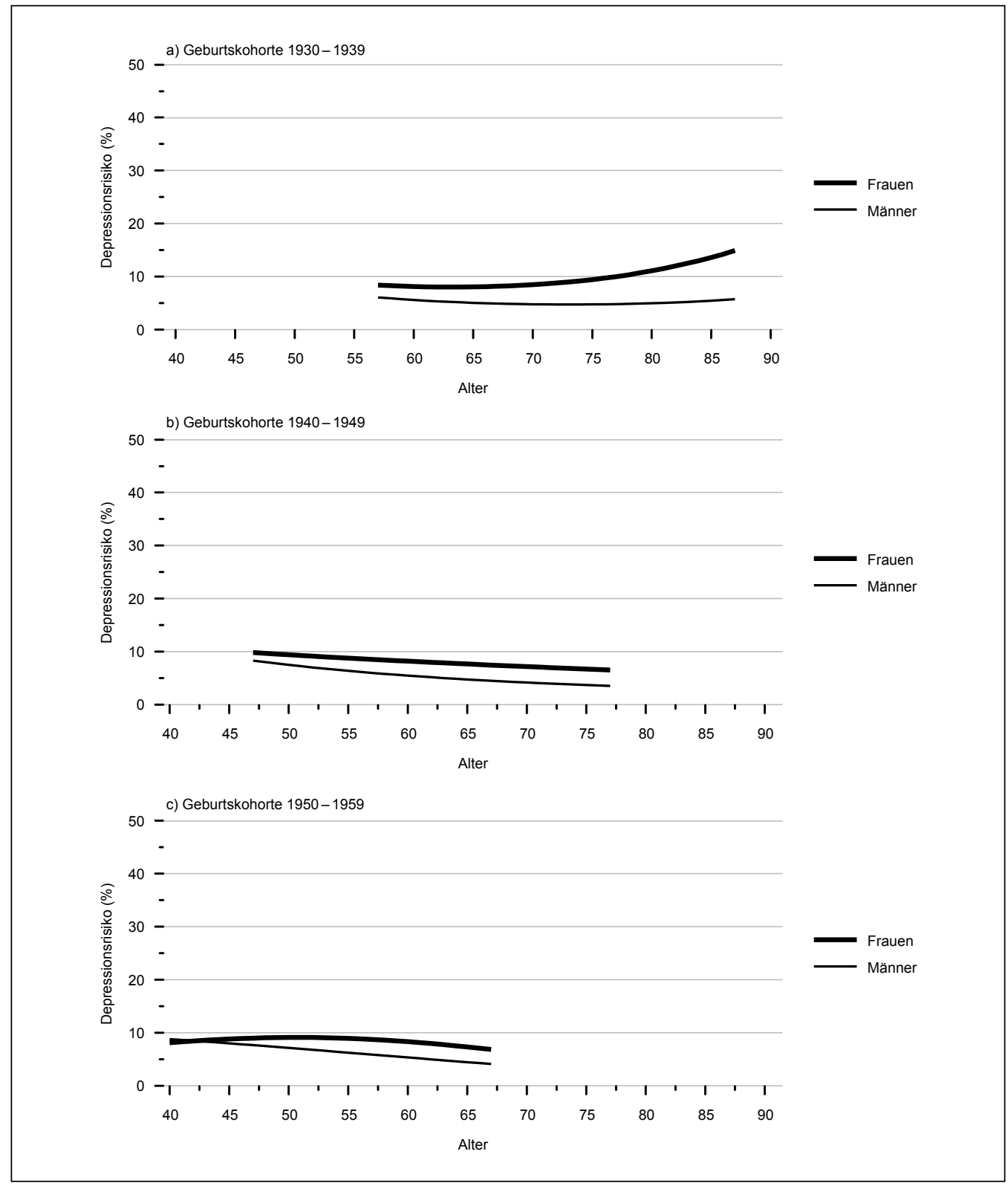

Quelle: DEAS 2002-2017; Schätzungen basieren auf $n=10504$ Studienteilnehmenden mit insgesamt $n=16677$ Beobachtungen; kontrolliert für Region. Der Wertebereich des Depressionsrisikos reicht von null Prozent bis 100 Prozent, in der Abbildung wird nur der Bereich null Prozent bis 50 Prozent gezeigt.

Die Kohorten unterscheiden sich nicht signifikant im Alternsverlauf des Depressionsrisikos, dies gilt für Frauen und Männer gleichermaßen. Somit bleiben die Geschlechterunterschiede im Alternsverlauf bestehen und verändern sich nicht signifikant über die Kohorten. 


\subsection{Diskussion}

\section{Zusammenfassung}

In diesem Kapitel wurden die Alternsverläufe von Lebenszufriedenheit und depressiven Symptomen, die zentrale Maße des allgemeinen Wohlbefindens und der subjektiven Lebensqualität sind, über die zweite Lebenshälfte für Frauen und Männer sowie die Rolle von geschlechtsspezifischen Kohortenunterschieden untersucht.

Bei Frauen und Männern folgen die durchschnittlichen Alternsverläufe von Lebenszufriedenheit und depressiven Symptomen zunächst einem positiven Trend vom 40. Lebensjahr an (also ein Zuwachs der Lebenszufriedenheit sowie ein Rückgang des Risikos klinisch auffälliger depressiver Symptome). Ab dem höheren Alter sinkt die durchschnittliche Lebenszufriedenheit, und das Depressionsrisiko steigt an. Frauen berichten, wenn sie das 64. Lebensjahr erreichen, eine höhere Lebenszufriedenheit als Männer, jedoch ist bei ihnen der durchschnittliche Rückgang der Lebenszufriedenheit im höheren Alter stärker als bei Männern. Zudem weisen Frauen über die gesamte zweite Lebenshälfte ein höheres Depressionsrisiko auf als Männer, das bei ihnen im höheren Alter auch stärker ansteigt als bei Männern. Was Kohortenunterschiede betrifft, legen unsere Befunde nahe, dass die später geborenen Kohorten mit einer höheren Lebenszufriedenheit das hohe und sehr hohe Alter erreichen werden, während der Alternsverlauf des Depressionsrisikos nicht unterschiedlich für die Kohorten ausgefallen ist, weder für Frauen noch für Männer.

\section{Interpretation}

Die jungen Alten (Baltes \& Smith 2003), also Personen um das 60. Lebensjahr, scheinen diejenigen zu sein, die im Durchschnitt das höchste psychische Wohlbefinden aufweisen (Mroczek \& Spiro 2005). Andere Studien beobachten ebenfalls, dass die mittlere Lebenszufriedenheit im mittle- ren Erwachsenenalter niedriger und die Depressionswahrscheinlichkeit größer ist als im höheren Alter von 60 Jahren (Blanchflower \& Oswald 2008; McAdams, Lucas, \& Donnellan 2012; Sutin et al. 2013b). Möglicherweise ist also das mittlere Erwachsenenalter eine belastungsreiche Phase für Frauen und Männer, in der Anforderungen aus verschiedenen Lebensbereichen wie Arbeit und Familie kumulieren und Zielkonflikte zwischen diesen Lebensbereichen auftreten können (Knecht, Bauer, Gutzwiller, \& Hämmig 2011). Dies kann eine Gefährdung der Work-Life-Balance und in der Folge auch des psychischen Wohlbefindens darstellen.

Dass im Durchschnitt die maximale Lebenszufriedenheit in einer Altersphase um das 65. Lebensjahr und später erreicht wird, kann zudem daran liegen, dass bei Frauen und Männern der Übergang in den Ruhestand, der ja bei einigen um dieses Alter herum vollzogen wird, zunächst mit einem Anstieg der Lebenszufriedenheit verknüpft ist (Wetzel, Huxhold, \& Tesch-Römer 2016), was Atchley (1976) als „Honeymoon-Phase“ bezeichnete.

Gleichzeitig ist es wichtig zu bedenken, dass die individuellen Alternsverläufe der Lebenszufriedenheit und der depressiven Symptome sehr unterschiedlich ausfallen von Frau zu Frau und von Mann zu Mann. Der beschriebene Verlauf der Lebenszufriedenheit und der Depression entspricht dem durchschnittlichen Veränderungstrend. Es werden also nicht alle Menschen mit einem Anstieg ihrer Lebenszufriedenheit oder einem Rückgang depressiver Symptome ab dem 40. Lebensjahr rechnen können, bei manchen wird die Lebenszufriedenheit stattdessen stabil bleiben oder sogar zurückgehen. Das gilt auch für spätere Lebensphasen: Im Übergang vom höheren ins sehr hohe Alter wird nicht jede Person zwingend einen Rückgang ihrer Lebenszufriedenheit oder einen Anstieg ihrer depressiven Symptomatik erleben.

Zudem halten der durchschnittliche Anstieg der Lebenszufriedenheit sowie der Rückgang der De- 
pressionswahrscheinlichkeit nicht über die gesamte Lebensspanne an. Vielmehr treten im Durchschnitt ca. zwischen dem 75. und 80. Lebensjahr Rückgänge in der Lebenszufriedenheit auf, während die Depressionswahrscheinlichkeit sogar bereits früher, nämlich um das 60 . Lebensjahr, ansteigt. Dass Lebenszufriedenheit im höheren Alter zurückgeht (mit besonders deutlichen Einbußen in den letzten 3 bis 5 Lebensjahren; Gerstorf et al. 2010), steht im Einklang mit der bisherigen Forschung (Mroczek \& Spiro 2005; Schilling 2006). Ebenso ist belegt, dass es im hohen und sehr hohen Alter eine erhebliche Gruppe von Frauen und Männern gibt, die mit einem Anstieg depressiver Symptome konfrontiert sind (Diegelmann et al. 2016; Schupp, Goebel, Kroh, \& Wagner 2013; Wettstein et al. 2015). Das sehr hohe Alter stellt in vielerlei Hinsicht eine verletzliche Lebensphase dar (Baltes \& Smith 2003; Schilling \& Wahl 2014; Wahl \& Schilling 2012), etwa in Bezug auf das Risiko für Demenz oder Einbußen der funktionalen Gesundheit (siehe Kapitel 3 Funktionale und subjektive Gesundheit in diesem Band), was eine geringere Lebenszufriedenheit sowie ein erhöhtes Depressionsrisiko in dieser Lebensphase erklären könnte.

Gleichzeitig kann jedoch, zumindest für die Lebenszufriedenheit, „Entwarnung“ gegeben werden: Unsere Befunde zeigen auch, dass die meisten Frauen und Männer mit ihrem Leben zufrieden sind und es bis ins sehr hohe Alter bleiben. In jedem Lebensalter über den Alternsverlauf liegt die durchschnittliche Lebenszufriedenheit über dem theoretischen Skalenmittelwert von 3. Zwar sinkt nach dem 75 . bis 80 . Lebensjahr die Lebenszufriedenheit im Durchschnitt ab, dieser Effekt ist jedoch - in Skaleneinheiten betrachtet - insgesamt klein.

Die durchschnittlichen Alternsverläufe in Lebenszufriedenheit und depressiver Symptomatik sind nicht identisch für Frauen und Männer: Frauen sind zwar um das 64. Lebensjahr im Durchschnitt zunächst zufriedener mit ihrem Leben als Männer. Allerdings ist der im höheren Alter auftretende mittlere Rückgang in der Lebenszufrie- denheit bei Frauen stärker als bei Männern. Gerade im hohen und sehr hohen Alter sind Frauen also weniger zufrieden als Männer. Ähnlich zeigt sich für das Risiko depressiver Symptome, dass dieses allgemein bei Frauen höher ist als bei Männern. Zudem steigt dieses Risiko bei Frauen stärker und schon früher im Alternsverlauf an als bei Männern. Somit stellen Frauen eine Risikogruppe dar, die von ungünstigeren Verläufen der Lebenszufriedenheit und der depressiven Symptome im hohen und sehr hohen Alter betroffen sind und daher möglicherweise besonders von Maßnahmen und Interventionen zum Erhalt der Lebensqualität bis ins sehr hohe Alter profitieren könnten.

Naheliegend ist die Vermutung, dass die beschriebenen Geschlechterunterschiede daran liegen, dass Frauen im Allgemeinen eine deutlichere Verschlechterung der funktionalen Gesundheit mit dem Alter erleben als Männer (siehe Kapitel 3 Funktionale und subjektive Gesundheit in diesem Band), was in der Folge stärkere Einbußen in der Lebenszufriedenheit sowie einen deutlicheren Anstieg depressiver Symptomatik im hohen Alter mit sich bringen könnte. Möglicherweise ist Verwitwung ein weiterer Erklärungsfaktor, denn sie stellt eine bedeutsame Gefährdung des psychischen Wohlbefindens dar (Luhmann, Hofmann, Eid, \& Lucas 2012), von der Frauen im Alter aufgrund ihrer höheren Lebenserwartung häufiger betroffen sind als Männer (Statistisches Bundesamt 2014). Darüber hinaus könnten Einkommensunterschiede zwischen Frauen und Männern und eine höhere Armutsgefährdung der Frauen im Alter (Lejeune, Romeu Gordo, \& Simonson 2017) oder Geschlechterunterschiede in anderen Ressourcen und Aufgaben (siehe Kapitel 6 Unbezahlte Sorgetätigkeiten und Kapitel 7 Ehrenamtliches Engagement in diesem Band) eine Rolle für die geschlechterdifferenziellen Alternsverläufe der Lebenszufriedenheit und der depressiven Symptomatik spielen.

Was Kohortenunterschiede betrifft, fällt der voraussichtliche Alternsverlauf bis ins hohe Alter für die Lebenszufriedenheit bei den später geborenen Kohorten - für Frauen und Männer gleichermaßen - positiver aus. Während also bei der 
früher geborenen Kohorte (1930-1939 Geborene) ein mittlerer Rückgang der Lebenszufriedenheit im hohen Alter beobachtet wird, wird ein solcher Rückgang nicht, oder in deutlich abgeschwächter Form, erwartet für die später geborenen Kohorten. Naheliegend und plausibel ist, dass veränderte Lebensbedingungen, etwa technischer Fortschritt, positive Kohortentrends in der Lebenszufriedenheit mit sich gebracht haben könnten. Positive Kohortentrends in Lebenszufriedenheit und anderen Wohlbefindensmaßen wurden - zumindest teilweise - auch in anderen Studien berichtet (Gerstorf, Hülür, Drewelies, Eibich, Duezel, Demuth, Ghisletta, Steinhagen-Thiessen, Wagner, \& Lindenberger 2015; Hülür, Drewelies, Eibich, Düzel, Demuth, Ghisletta, Steinhagen-Thiessen, Wagner, Lindenberger, \& Gerstorf 2016; Hülür et al. 2015; Sutin et al. 2013a).

Derartige in die Zukunft gerichtete Verlaufsschätzungen könnten sich für die später geborenen Kohorten womöglich als unzutreffend erweisen, sie setzen voraus, dass sich bisherige Kohortentrends fortsetzen werden. Betrachtet wurden zudem in diesen Analysen nur drei Geburtskohorten, die relativ nahe beieinander liegen. Kohortentrends mögen stärker (und womöglich auch stärker geschlechterdifferenziell) ausfallen, wenn chronologisch weiter auseinander liegende Geburtsjahrgänge betrachtet werden, und dies über einen (noch) ausgedehnteren Beobachtungszeitraum.

Anders als bei der Lebenszufriedenheit zeigt sich für das Risiko depressiver Symptome kein positiver Kohortentrend. Dieser Befund legt nahe, dass noch mehr in eine Verbesserung gesellschaftlicher Rahmenbedingungen investiert werden muss, damit künftige Ältere nicht nur mit einer höheren Lebenszufriedenheit, sondern auch mit einem geringeren Depressionsrisiko ins hohe und sehr hohe Alter kommen. Das gilt besonders für Frauen, denn dieser nicht beobachtbare Kohortentrend bedeutet auch, dass die diskrepanten Alternsverläufe im Depressionsrisiko von Frauen und Männern über die Kohorten stabil geblieben sind. Auch künftig müssen also Frauen im Übergang vom hohen ins sehr hohe Alter wohl damit rech- nen, dass ihr Depressionsrisiko stärker ansteigt als das der Männer. Faktoren, die der gesellschaftliche Wandel mit sich gebracht hat, etwa veränderte Lebens- und Arbeitsbedingungen, mögen also positiv beeinflusst haben, wie sich Frauen und Männer unterschiedlicher Geburtsjahrgänge hinsichtlich ihrer Lebenszufriedenheit entwickelt haben, nicht jedoch, wie sich ihr Depressionsrisiko im Alternsverlauf ändert. Das Depressionsrisiko ist insbesondere im hohen und sehr hohen Alter stark von Aspekten der körperlichen Gesundheit einer Person abhängig (Diegelmann et al. 2016; Schilling, Wahl, \& Reidick 2013). Befunde zur Gesundheit (siehe Kapitel 3 Funktionale und subjektive Gesundheit in diesem Band) legen nahe, dass sich der ungünstigere durchschnittliche Alternsverlauf der funktionalen Gesundheit bei Frauen im Vergleich zu Männern ebenfalls über nachfolgende Kohorten fortgesetzt hat beziehungsweise fortsetzen wird. Die Diskrepanz im gesundheitlichen Alternsverlauf von Frauen und Männern hat also nicht abgenommen, und das könnte eine Erklärung dafür sein, dass auch die diskrepanten Alternsverläufe der depressiven Symptomatik bei Frauen und Männern stabil geblieben sind.

\section{Politische Implikationen}

Anders als für die Lebenszufriedenheit gibt es für das Depressionsrisiko auf Grundlage unserer Ergebnisse keinen Hinweis darauf, dass die Alternsverläufe sozialem Wandel unterliegen und für später Geborene günstiger ausfallen werden. Somit müssen auch später geborene Jahrgänge damit rechnen, dass ihr Depressionsrisiko im hohen und sehr hohen Alter ansteigt, und zwar in ähnlicher Weise wie bei früher geborenen Jahrgängen. Offenbar gibt es also noch Handlungsbedarf, damit Menschen künftig mit einem geringeren Risiko für ausgeprägte depressive Symptome das hohe und sehr hohe Alter erreichen. Insbesondere die Gesundheit als ein bedeutsamer Einflussfaktor auf depressive Symptomatik sollte dabei in den Blick genommen werden. Dabei ist zu bedenken, dass Angebote der 
Gesundheitsförderung und der Prävention von Erkrankungen nicht auf spätere Lebensphasen begrenzt sein sollten, sondern auch schon in früheren Lebensphasen wichtig sind, um Menschen zu einem „gesunden Altern“ zu verhelfen.

Zudem müssen, um möglichst vielen Menschen ein „gutes Leben“ in der zweiten Lebenshälfte zu ermöglichen, gemäß unserer Befunde vor allem die Gruppe sehr alter Erwachsener - besonders sehr alter Frauen - sowie die offenbar ebenfalls belastete und gefährdete Gruppe der Frauen und Männer im mittleren Erwachsenenalter in den Blick genommen werden. Im mittleren Erwachsenenalter könnten Maßnahmen und Interventionen vielversprechend sein, welche die Work-Life-Balance erleichtern. Alarmierend ist, dass der beschrie- bene ausbleibende Wandel im Alternsverlauf des Depressionsrisikos für Frauen und Männer gleichermaßen gilt. Die deutlichere Gefährdung von Frauen im Vergleich zu Männern, im hohen Alter unter depressiven Symptomen zu leiden, wird also in später geborenen Jahrgängen voraussichtlich bestehen bleiben. Umso wichtiger könnten daher Interventionen sein, um die Gesundheit der Frauen im hohen und sehr hohen Alter - und auch bereits in früheren Lebensaltern - zu erhalten oder $\mathrm{zu}$ steigern. Dies gilt ebenso für weitere Maßnahmen, die auf die Prävention von Depression - besonders bei Frauen - abzielen. Diese könnten einen erheblichen Beitrag leisten, damit Frauen künftig mit einem geringeren Depressionsrisiko alt werden.

\section{Literatur}

Atchley, R. C. (1976). The sociology of retirement. New York: Halsted Press.

Baltes, P. B., \& Smith, J. (2003). New Frontiers in the Future of Aging: From Successful Aging of the Young Old to the Dilemmas of the Fourth Age. Gerontology, 49(2), 123-135.

Blanchflower, D. G., \& Oswald, A. J. (2008). Is well-being U-shaped over the life cycle? Social Science \& Medicine, 66(8), 1733-1749. doi: 10.1016/j.socscimed.2008.01.030.

Brault, M.-C., Meuleman, B., \& Bracke, P. (2012). Depressive symptoms in the Belgian population: disentangling age and cohort effects. Social Psychiatry and Psychiatric Epidemiology, 47(6), 903-915. doi: 10.1007/s00127-011-0398-0.

Diegelmann, M., Schilling, O. K., \& Wahl, H.-W. (2016). Feeling blue at the end of life: Trajectories of depressive symptoms from a distance-to-death perspective. Psychology and Aging, 31(7), 672-686. doi: 10.1037/pag0000114.

Diener, E., Emmons, R. A., Larsen, R. J., \& Griffin, S. (1985). The Satisfaction With Life Scale. Journal of Personality Assessment, 49(1), 71-75. doi: 10.1207/s15327752jpa4901_13.

Diener, E., Suh, E. M., Lucas, R. E., \& Smith, H. L. (1999). Subjective well-being: Three decades of progress. Psychological Bulletin, 125(2), 276-302. doi: 10.1037/0033-2909.125.2.276.

Gerstorf, D., Hülür, G., Drewelies, J., Eibich, P., Duezel, S., Demuth, I., Ghisletta, P., Steinhagen-Thiessen, E., Wagner, G. G., \& Lindenberger, U. (2015). Secular changes in late-life cognition and well-being: Towards a long bright future with a short brisk ending? Psychology and Aging, 30(2), 301-310. doi: 10.1037/pag0000016.

Gerstorf, D., Ram, N., Mayraz, G., Hidajat, M., Lindenberger, U., Wagner, G. G., \& Schupp, J. (2010). LateLife Decline in Well-Being Across Adulthood in Germany, the UK, and the US: Something is Seriously Wrong at the End of Life. Psychology and Aging, 25(2), 477-485. doi: 10.1037/a0017543. 
Glaesmer, H., Grande, G., Braehler, E., \& Roth, M. (2011). The German version of the satisfaction with life scale (SWLS). European Journal of Psychological Assessment, 27(2), 127-132. doi: 10.1027/1015-5759/ a000058.

Hautzinger, M., \& Bailer, M. (1993). ADS - Allgemeine Depressions Skala. Weinheim: Beltz.

Herschbach, P. (2002). Das „Zufriedenheitsparadox" in der Lebensqualitätsforschung. Psychotherapie, Psychosomatik und Medizinische Psychologie, 52(03/04), 141-150. doi: 10.1055/s-2002-24953.

Hülür, G., Drewelies, J., Eibich, P., Düzel, S., Demuth, I., Ghisletta, P., Steinhagen-Thiessen, E., Wagner, G. G., Lindenberger, U., \& Gerstorf, D. (2016). Cohort differences in psychosocial function over 20 years: current older adults feel less lonely and less dependent on external circumstances. Gerontology, 62(3), 354-361. doi: 10.1159/000438991.

Hülür, G., Ram, N., \& Gerstorf, D. (2015). Historical improvements in well-being do not hold in late life: Birth- and death-year cohorts in the United States and Germany. Developmental Psychology, 51(7), 998-1012. doi: 10.1037/a0039349.

Kasen, S., Cohen, P., Chen, H., \& Castille, D. (2003). Depression in adult women: age changes and cohort effects. American Journal of Public Health, 93(12), 2061-2066.

Knecht, M. K., Bauer, G. F., Gutzwiller, F., \& Hämmig, O. (2011). Persistent work-life conflict and health satisfaction - A representative longitudinal study in Switzerland. BMC Public Health, 11(271), 1-8. doi: 10.1186/1471-2458-11-271.

Lehr, D., Hillert, A., Schmitz, E., \& Sosnowsky, N. (2008). Screening depressiver Störungen mittels Allgemeiner Depressions-Skala (ADS-K) und State-Trait Depressions Scales (STDS-T). Diagnostica, 54(2), 61-70.

Lejeune, C., Romeu Gordo, L., \& Simonson, J. (2017). Einkommen und Armut in Deutschland: Objektive Einkommenssituation und deren subjektive Bewertung. In: K. Mahne, J. K. Wolff, J. Simonson \& C. Tesch-Römer (Hrsg.) Altern im Wandel. Zwei Jahrzehnte Deutscher Alterssurvey (DEAS) (S. 97-110). Wiesbaden: Springer VS.

Luhmann, M., Hofmann, W., Eid, M., \& Lucas, R. E. (2012). Subjective well-being and adaptation to life events: a meta-analysis. Journal of Personality and Social Psychology, 102(3), 592-615.

McAdams, K. K., Lucas, R. E., \& Donnellan, M. B. (2012). The Role of Domain Satisfaction in Explaining the Paradoxical Association Between Life Satisfaction and Age. Social indicators research, 109(2), 295303. doi: 10.1007/s11205-011-9903-9.

Mroczek, D. K., \& Spiro, A., III. (2005). Change in Life Satisfaction During Adulthood: Findings From the Veterans Affairs Normative Aging Study. Journal of Personality and Social Psychology, 88(1), 189-202. doi: 10.1037/0022-3514.88.1.189.

Murphy, J. M., Laird, N. M., Monson, R. R., Sobol, A. M., \& Leighton, A. H. (2000). A 40-year perspective on the prevalence of depression: the Stirling County Study. Archives of General Psychiatry, 57(3), 209-215.

Pinquart, M., \& Sörensen, S. (2001). Gender differences in self-concept and psychological well-being in old age: a meta-analysis. The Journals of Gerontology, Series B: Psychological Sciences and Social Sciences, 56(4), 195-213.

Radloff, L. S. (1977). The CES-D Scale: A Self-Report Depression Scale for Research in the General Population. Applied Psychological Measurement, 1(3), 385-401. doi: 10.1177/014662167700100306.

Ryan, R. M., \& Deci, E. L. (2001). On Happiness and Human Potentials: A Review of Research on Hedonic and Eudaimonic Well-Being. Annual Review of Psychology, 52(1), 141-166. doi: 10.1146/annurev. psych.52.1.141. 
Ryff, C. D., Dienberg Love, G., Urry, H. L., Muller, D., Rosenkranz, M. A., Friedman, E. M., Davidson, R. J., \& Singer, B. (2006). Psychological Well-Being and III-Being: Do They Have Distinct or Mirrored Biological Correlates? Psychotherapy and Psychosomatics, 75(2), 85-95. doi: 10.1159/000090892.

Schilling, O. K. (2006). Development of Life Satisfaction in Old Age: Another view on the ,paradox'. Social Indicators Research, 75(2), 241-271. doi: 10.1007/s11205-004-5297-2.

Schilling, O. K. (2005). Cohort- and age-related decline in elder's life satisfaction: is there really a paradox? European Journal of Ageing, 2(4), 254-263. doi: 10.1007/s10433-005-0016-7.

Schilling, O. K., \& Wahl, H.-W. (2014). Herausforderungen am Ende der Lebensspanne - Facetten von Hochaltrigkeit zwischen bedeutsamer Anpassung und hoher Verletzlichkeit. In: H.-W. Wahl \& A. Kruse (Hrsg.) Lebensläufe im Wandel (S. 201-214). Stuttgart: Kohlhammer.

Schilling, O. K., Wahl, H.-W., \& Reidick, O. (2013). Trajectories of depressive symptoms in advanced old age: A functional approach concerning the role of physical functioning. GeroPsych: The Journal of Gerontopsychology and Geriatric Psychiatry, 26(1), 29-38. doi: 10.1024/1662-9647/a000079.

Schupp, J., Goebel, J., Kroh, M., \& Wagner, G. G. (2013). Zufriedenheit in Deutschland so hoch wie nie nach der Wiedervereinigung: Ostdeutsche signifikant unzufriedener als Westdeutsche. DIW Wochenbericht, 80(47), 34-43.

Shao, J., Li, D., Zhang, D., Zhang, L., Zhang, Q., \& Qi, X. (2013). Birth cohort changes in the depressive symptoms of Chinese older adults: a cross-temporal meta-analysis. International Journal of Geriatric Psychiatry, 28(11), 1101-1108. doi: 10.1002/gps.3942.

Spuling, S., Ziegelmann, J. P., \& Wünsche, J. (2017). Was tun wir für unsere Gesundheit? Gesundheitsverhalten in der zweiten Lebenshälfte. In: K. Mahne, J. K. Wolff, J. Simonson \& C. Tesch-Römer (Hrsg.) Altern im Wandel. Zwei Jahrzehnte Deutscher Alterssurvey (DEAS). Wiesbaden: Springer VS.

Statistisches Bundesamt (2014). Haushalte und Familien - Ergebnisse des Mikrozensus: Bevölkerung und Erwerbstätigkeit. Wiesbaden: Statistisches Bundesamt.

Staudinger, U. M. (2000). Viele Gründe sprechen dagegen, und trotzdem geht es vielen Menschen gut: Das Paradox des subjektiven Wohlbefindens. Psychologische Rundschau, 51(4), 185-197. doi: 10.1026//0033-3042.51.4.185

Sutin, A. R., Terracciano, A., Milaneschi, Y., An, Y., Ferrucci, L., \& Zonderman, A. B. (2013a). The effect of birth cohort on well-being: the legacy of economic hard times. Psychological Science, 24(3), 379-385. doi: $10.1177 / 0956797612459658$.

Sutin, A. R., Terracciano, A., Milaneschi, Y., An, Y., Ferrucci, L., \& Zonderman, A. B. (2013b). The trajectory of depressive symptoms across the adult lifespan. JAMA psychiatry, 70(8), 803-811. doi: 10.1001/ jamapsychiatry.2013.193.

Tesch-Römer, C., Motel-Klingebiel, A., \& Tomasik, M. J. (2008). Gender Differences in Subjective Well-Being: Comparing Societies with Respect to Gender Equality. Social Indicators Research, 85(2), 329-349. doi: 10.1007/s11205-007-9133-3.

Wahl, H.-W., \& Schilling, O. (2012). Das hohe Alter. In: W. Schneider \& U. Lindenberger (Hrsg.) Entwicklungspsychologie (S. 311-336). Weinheim: Beltz Verlag.

Wettstein, M., Schilling, O. K., Reidick, O., \& Wahl, H.-W. (2015). Four-year stability, change, and multidirectionality of well-being in very-old age. Psychology and Aging, 30(3), 500-516. doi: 10.1037/ pag0000037.

Wetzel, M., Huxhold, O., \& Tesch-Römer, C. (2016). Transition into Retirement Affects Life Satisfaction: Short-and Long-Term Development Depends on Last Labor Market Status and Education. Social Indicators Research, 125(3), 991-1009. doi: 10.1007/s11205-015-0862-4. 
Wolff, J. K., \& Tesch-Römer, C. (2017). Glücklich bis ins hohe Alter? Lebenszufriedenheit und depressive Symptome in der zweiten Lebenshälfte. In: K. Mahne, J. K. Wolff, J. Simonson \& C. Tesch-Römer (Hrsg.) Altern im Wandel. Zwei Jahrzehnte Deutscher Alterssurvey (DEAS) (S. 171-183). Wiesbaden: Springer VS.

Yang, Y. (2007). Is old age depressing? Growth trajectories and cohort variations in late-life depression. Journal of Health and Social Behavior, 48(1), 16-32.

Open Access Dieses Kapitel wird unter der Creative Commons Namensnennung 4.0 International Lizenz (http:// creativecommons.org/licenses/by/4.0/deed.de) veröffentlicht, welche die Nutzung, Vervielfältigung, Bearbeitung, Verbreitung und Wiedergabe in jeglichem Medium und Format erlaubt, sofern Sie den/die ursprünglichen Autor(en) und die Quelle ordnungsgemäß nennen, einen Link zur Creative Commons Lizenz beifügen und angeben, ob Änderungen vorgenommen wurden.

Die in diesem Kapitel enthaltenen Bilder und sonstiges Drittmaterial unterliegen ebenfalls der genannten Creative Commons Lizenz, sofern sich aus der Abbildungslegende nichts anderes ergibt. Sofern das betreffende Material nicht unter der genannten Creative Commons Lizenz steht und die betreffende Handlung nicht nach gesetzlichen Vorschriften erlaubt ist, ist für die oben aufgeführten Weiterverwendungen des Materials die Einwilligung des jeweiligen Rechteinhabers einzuholen. 


\title{
5. Soziale Isolation und Einsamkeit bei Frauen und Männern im Verlauf der zweiten Lebenshälfte
}

\author{
Oliver Huxhold und Heribert Engstler
}

\section{Kernaussagen}

Die Risiken sozialer Isolation und Einsamkeit sind unterschiedlich hoch und entwickeln sich mit dem Älterwerden im Verlauf der zweiten Lebenshälfte verschieden: Während das Isolationsrisiko zwischen dem 40. und 90. Lebensjahr relativ kontinuierlich von vier auf 22 Prozent steigt, verläuft das Einsamkeitsrisiko u-förmig. Vom Alter 40 bis Mitte 60 sinkt es, danach nimmt es wieder zu, ist aber selbst im sehr hohen Alter, mit 90 Jahren, mit rund elf Prozent nicht höher als mit 40 Jahren.

Das Isolationsrisiko unterscheidet sich im Alternsverlauf zwischen Frauen und Männern: Bei Männern steigt das Risiko sozialer Isolation über die betrachtete Altersspanne zwischen 40 und 90 Jahren relativ gleichmäßig von fünf auf 20 Prozent an. Frauen erleben zunächst einen schwächeren Risikoanstieg, der sich im Rentenalter jedoch beschleunigt, so dass sie im Alter ab Ende 70 ähnlich häufig sozial isoliert sind wie Männer. Zuvor haben Frauen mehr als drei Lebensjahrzehnte lang vom Alter Anfang 40 bis Mitte 70 ein geringeres Isolationsrisiko als Männer.

Auch das Einsamkeitsrisiko unterscheidet sich im Alternsverlauf zwischen Frauen und Männern: Im mittleren Erwachsenenalter zwischen 40 und 60 Jahren sind Männer etwas häufiger einsam als Frauen. Der Geschlechterunterschied nimmt mit steigendem Alter jedoch ab und dreht sich im Verlauf des Rentenalters um, so dass im hohen Alter mehr Frauen als Männer einsam sind. Mit 90 Jahren haben Frauen ein Risiko von 14 Prozent einsam zu sein. Bei Männern in diesem Alter beträgt das Einsamkeitsrisiko neun Prozent.

Bei jüngeren Geburtskohorten steigt das Isolationsrisiko mit dem Älterwerden nicht mehr so hoch an wie bei den älteren Geburtsjahrgängen. Das Einsamkeitsrisiko ist in den später geborenen Jahrgängen weniger stark mit dem Älterwerden verknüpft. Der soziale Wandel hat die Unterschiede zwischen Frauen und Männern nicht verändert: Während bei den 1930 bis 1939 geborenen Menschen das Isolationsrisiko bis in das Alter von Mitte 70 stieg, endet der Altersanstieg des Isolationsrisikos bei den 1950 bis 1959 Geborenen vorläufig bereits im Alter Mitte 60 auf einem niedrigeren Niveau. Beim Einsamkeitsrisiko zeigt sich für die 1950 bis 1959 Geborenen ein niedrigeres Ausgangsniveau im mittleren Erwachsenenalter und eine Abflachung des u-förmigen Alternsverlaufs. Voraussichtlich werden die dann 70- bis 80-Jährigen nicht häufiger einsam oder sozial isoliert sein als die heutigen 70- bis 80-Jährigen. Zudem ist bislang keine Angleichung in den Alternsverläufen zwischen Frauen und Männern erkennbar. 


\subsection{Einleitung}

Mit anderen Menschen verbunden zu sein, das eigene Leben mit anderen $\mathrm{zu}$ teilen, gute und enge Beziehungen zu haben, ist allen Frauen und Männern ein angeborenes Bedürfnis (Baumeister \& Leary 1995). Aus diesem Grund sind soziale Isolation und Einsamkeit für viele Menschen zutiefst stressvolle Erfahrungen, die mit erheblichen Gesundheitsrisiken verbunden sind (Hawkley \& Cacioppo 2010). Insbesondere lange Phasen der Einsamkeit erhöhen unter anderem das Risiko von Schlafstörungen, Depressionen und kardiovaskulären Erkrankungen und gehen letztlich sogar mit einem erhöhten Mortalitätsrisiko einher (Luo, Hawkley, Waite, \& Cacioppo 2012).

In der öffentlichen Debatte werden die Begriffe „Soziale Isolation“ und „Einsamkeit“ häufig synonym verwendet. Die Forschung hat jedoch wiederholt gezeigt, dass es sich um unterschiedliche Phänomene handelt (Hawkley \& Cacioppo 2010). Je nach Persönlichkeit unterscheiden sich Menschen darin, wie viel soziale Einbettung sie bevorzugen. So gibt es zum Beispiel Menschen, die sich auf eine enge Freundschaft konzentrieren, und solche, die einen großen Kreis aus mehr oder weniger engen Freunden und Bekannten pflegen (Miche, Huxhold, \& Stevens 2013). Im Gegensatz $\mathrm{zu}$ solchen gewünschten Unterschieden in der $\mathrm{Zu}$ sammensetzung und Beziehungsintensität sozialer Netzwerke zwischen Personen, bezeichnet soziale Isolation einen bedeutenden Mangel im Kontakt $\mathrm{zu}$ anderen Menschen. Sie kann von außen beobachtet werden, unabhängig von der subjektiven Bedeutung für das Individuum. Menschen, die viel Zeit allein verbringen und wenig mit anderen Personen interagieren, können als sozial isoliert bezeichnet werden (Hawthorne 2006). Einsamkeit ist hingegen eine sehr subjektive Erfahrung. Sie beschreibt das zutiefst unangenehme Gefühl, dass die sozialen Beziehungen, die man pflegt, und der persönliche Austausch mit anderen Menschen nicht den eigenen Bedürfnissen nach Zugehörigkeit und Geborgenheit entsprechen (Perlman \& Peplau 1981). Demzufolge haben Menschen, die sozial isoliert sind, zwar eine höhere Wahrscheinlichkeit sich einsam zu fühlen, es gibt aber auch viele Personen, die von sich aus mit nur sehr wenigen Menschen einen engen Austausch pflegen und mit dem Alleinsein gut zurechtkommen. Andererseits können Menschen sich einsam fühlen, die objektiv gesehen über viele soziale Kontakte verfügen, weil ihnen in diesen Beziehungen emotionale Tiefe und Verständnis fehlen.

In den letzten Jahrzehnten hat sich fast in allen westlichen Industrienationen eine starke öffentliche Debatte um die Themen soziale Isolation und Einsamkeit herausgebildet. Zwei Befürchtungen stehen innerhalb dieser Diskussion im Zentrum. Zum einen befürchten viele Menschen, dass im Alter das Risiko, sozial isoliert und/oder einsam zu sein, enorm hoch sei (Drinkuth 2018) - fast so als sei Einsamkeit eine garantierte Begleiterscheinung des hohen Erwachsenenalters. Zum anderen wird häufig die Meinung vertreten, dass der Anteil der Menschen, die unter sozialer Isolation und Einsamkeit leiden, in den letzten Jahren stark zugenommen habe (Drinkuth 2018; Will 2017). In einigen Veröffentlichungen wird sogar von einer "Epidemie der Einsamkeit“ gesprochen, die immer weitere Teile der Bevölkerung insbesondere unter den älteren Menschen erfassen wird (Drinkuth 2018; Will 2017). Doch entsprechen diese beiden häufig geäußerten Befürchtungen der Wirklichkeit? Nehmen die Risiken, sozial isoliert zu sein und einsam zu sein, wirklich mit steigendem $\mathrm{Al}$ ter zu?

Um die letztgenannte Frage beantworten zu können, bedarf es längsschnittlicher Analysen. Das Isolationsrisiko oder das Einsamkeitsrisiko für die gleichen Personen muss über einen längeren Zeitraum hinweg mehrfach gemessen werden, um beurteilen zu können, ob diese Risiken tatsächlich im Zuge des Älterwerdens ansteigen (siehe Kapitel 2 Design, Inhalte und Methodik des Deutschen Alterssurveys in diesem Band). Leider sind bisherige Forschungsarbeiten zum Zusammenhang zwischen Alter und Einsamkeit jedoch 
mit wenigen Ausnahmen (z. B. Böger \& Huxhold 2018) querschnittlicher Natur. Querschnittliche Analysen vergleichen das Ausmaß von Einsamkeit zwischen verschiedenen Altersgruppen zu einem einzigen Zeitpunkt. Aufgrund solcher Untersuchungen lässt sich nicht unterscheiden, ob eventuelle Altersgruppenunterschiede tatsächlich auf Effekte des Älterwerdens zurückzuführen sind oder ob sich diese Ungleichheiten auf Grund von unterschiedlichen Lebensbedingungen verschiedener Geburtskohorten ergeben. Zudem muss man bedenken, dass die Älteren keine homogene Gruppe sind. Lebensbedingungen, die zur Ausbildung eines erhöhten Risikos für soziale Isolation und für Einsamkeit führen können - wie zum Beispiel das Fehlen einer festen Partnerschaft -, unterscheiden sich beispielsweise zwischen Frauen und Männern und das in unterschiedlichem Maße in unterschiedlichen Lebensphasen.

\section{Unterschiede zwischen Frauen und Männern}

Generell ergab die bisherige Forschung relativ uneinheitliche Ergebnisse in Bezug auf Unterschiede im Ausmaß sozialer Integration zwischen Frauen und Männern. Während einige Studien zeigten, dass Frauen größere soziale Netzwerke pflegen als Männer (Suanet \& Huxhold 2018), fanden andere Untersuchungen wiederum keinerlei Unterschiede zwischen den Geschlechtern (Antonucci, Lansford, Akiyama, Smith, Baltes, Takahashi, Fuhrer, \& Dartigues 2002). Auch bezüglich der Geschlechterunterschiede in der Verbreitung von Einsamkeit sind die Studienergebnisse nicht eindeutig und zudem abhängig von dem jeweils verwendeten Messinstrument (von Soest, Luhmann, Hansen, \& Gerstorf 2018). Dieses insgesamt uneinheitliche Bild lässt sich vermutlich dadurch erklären, dass die sozialen Beziehungen von Frauen und Männern gleichzeitig von verschiedenen Einflüssen gestaltet werden, die sich unterschiedlich auf das Isolationsund Einsamkeitsrisiko auswirken und in der Summe auch gegenseitig aufheben können. So sind auf der einen Seite im höheren Lebensalter Risi- kofaktoren für soziale Isolation und Einsamkeit wie beispielsweise einschränkende Krankheiten, Verwitwung und finanzielle Nöte bei Frauen stärker ausgeprägt als bei Männern (Antonucci et al. 2002). Aus einer Ressourcenperspektive heraus würde man daher mutmaßen, dass sich Geschlechterunterschiede mit dem Alter vergrößern, da diese gesundheits- und verlustbezogenen Risiken für Einsamkeit und soziale Isolation für Frauen stärker zunehmen als für Männer. Auf der anderen Seite jedoch investieren Frauen stärker als Männer in soziale Beziehungen und pflegen häufigeren Kontakt zu ihren Familien und insbesondere $\mathrm{zu}$ ihren Freundinnen und Freunden (Sander, Schupp, \& Richter 2017). Frauen scheinen möglicherweise aus diesem Grund besser in der Lage zu sein, auch mit fortschreitendem Alter eher auf soziale Unterstützung aus ihrem sozialen Netzwerk zurückgreifen zu können als Männer (Fischer \& Beresford 2015). Betrachtet man also die Entwicklung der Risiken für soziale Isolation und Einsamkeit aus einer Perspektive, die diese kumulative Investition in soziale Netzwerke über die Lebensspanne berücksichtigt, so sind ganz im Gegensatz zu einer Ressourcenperspektive Männer mit fortschreitendem Alter eher gefährdet als Frauen. Diese Vermutung könnte insbesondere auf später geborene Geburtskohorten zutreffen, wie wir im nächsten Abschnitt darlegen werden.

\section{Wandel geschlechtsspezifischer Verläufe der Risiken von Einsamkeit und sozialer Isolation}

In der öffentlichen Debatte wird mitunter vor einer herannahenden „Epidemie der Einsamkeit“ gewarnt, also einer starken Zunahme der sozialen Isolation und Einsamkeit bei den später geborenen Geburtsjahrgängen (Drinkuth 2018). Begründet wird dies häufig mit dem beobachteten Anstieg von Kinderlosigkeit, angewachsenen Scheidungsraten und vergrößerten Wohnentfernungen zwischen Familienmitgliedern in den letzten Jahrzehnten (Mahne \& Huxhold 2017; Engstler \& Tesch-Römer 2010). Dabei wird in dieser Debatte häufig über- 
sehen, dass trotz der wachsenden Wohnentfernungen wahrscheinlich auch auf Grund der Nutzung moderner Kommunikationsmittel die Qualität der Familienbeziehungen sehr gut geblieben ist (Mahne \& Huxhold 2017). Zudem zeigt sich, dass die sozialen Netzwerke heutzutage viel stärker als früher Freundinnen und Freunde beinhalten und diese immer mehr soziale Unterstützungsleistungen übernehmen, die vormals Familienmitgliedern vorbehalten waren (Böger, Wetzel, \& Huxhold 2017; Huxhold, Mahne, \& Naumann 2010; Suanet \& Antonucci 2017). Eine Studie, die Geschlechterunterschiede über verschiedene Kohorten hinweg hinsichtlich der längsschnittlichen Entwicklung (über 17 Jahre) von Freundschaftsbeziehungen untersuchte, ist die Longitudinal Aging Study Amsterdam (LASA; Stevens \& van Tilburg 2011). Auch diese Studie bestätigte eine wachsende Bedeutung von Freundschaften und deren Aufrechterhaltung im Alter innerhalb jüngerer Kohorten und traf zudem Aussagen über typische Entwicklungsverläufe von Freundschaften für Frauen und Männer. So zeigten die Autorin und der Autor nicht nur, dass Frauen insgesamt mehr Freundschaften berichten als Männer und diese länger aufrechterhalten, sondern auch, dass dieser Geschlechterunterschied über die Kohorten hinweg zunahm. Diese Freundschaftsbeziehungen könnten Frauen nutzen, um eventuelle Lücken in Familienbeziehungen auszugleichen. Passend dazu fanden Perrig-Chiello und Kollegen (2015), dass nur bei verwitweten Frauen, aber nicht bei verwitweten Männern, ein Mangel an sozialen Kontakten im Jahr 2011 signifikant seltener berichtet wurde als bei einer befragten Kohorte im Jahr 1979 (Perrig-Chiello, Spahni, Hopflinger, \& Carr 2016). Die oben zitierten Befunde sprechen für die Vermutung, dass es Frauen insgesamt etwas besser als Männern gelungen ist, sich den veränderten Familienbeziehungen anzupassen. Über Geburtskohorten hinweg lässt sich aus dieser Perspektive erwarten, dass sich die Alternsverläufe der Risiken sozialer Isolation und Einsamkeit in später geborenen Geburtskohorten positiver für Frauen als für Männer entwickeln.

\section{Fragestellungen}

Im Zentrum dieses Kapitels stehen die Fragen, wie sich mit dem Älterwerden im Verlauf der zweiten Lebenshälfte die Risiken sozial isoliert und/oder einsam zu sein, entwickeln und ob diese Risiken für Frauen und Männer unterschiedlich sind. Darüber hinaus wird geprüft, ob es in Deutschland einen gesellschaftlichen Wandel zu mehr Isolation und Einsamkeit im Alter gibt und ob sich dieser bei Frauen und Männern unterscheidet. Der Beitrag beschreitet in mehrerer Hinsicht Neuland. Zunächst werden erstmalig längsschnittliche Entwicklungsverläufe hinsichtlich der Risiken für soziale Isolation und Einsamkeit getrennt für Frauen und Männer betrachtet und nicht nur Altersgruppen miteinander verglichen. Hierbei gehen wir davon aus, dass Frauen im mittleren Lebensalter möglicherweise im Vorteil sind, da sie tendenziell stärker in ihre sozialen Netze investieren. Mit steigendem Lebensalter und einer möglichen $\mathrm{Zu}$ nahme von funktionalen Gesundheitseinschränkungen sowie einem erhöhten Verwitwungsrisiko könnte sich dieser Vorteil jedoch abschwächen, da Frauen häufiger als Männer im höheren Alter von diesen negativen Entwicklungen und Lebensereignissen betroffen sind. Zudem könnten Frauen den historischen Wandel in den Familien- und Paarbeziehungen möglicherweise besser bewältigen als Männer, da sie in einem stärkeren Maße Freundschaften pflegen. Geschlechterunterschiede in den längsschnittlichen Alternsverläufen von sozialer Isolation und Einsamkeit könnten sich dementsprechend zu Gunsten von Frauen verlagern. 


\subsection{Methoden}

\section{Stichprobe}

Die empirische Untersuchung der geschlechtsspezifischen Alternsverläufe des Einsamkeitsrisikos und des Risikos sozialer Isolation stützt sich auf die Paneldaten des Deutschen Alterssurveys (DEAS) der Jahre 1996 bis 2017. In den Jahren 1996, 2002, 2008 und 2014 wurden jeweils unabhängige, für Deutschland repräsentative Einwohnermeldeamtsstichproben der in Privathaushalten lebenden 40- bis 85-jährigen Bevölkerung gezogen, die nach ihrer ersten Befragung in den Längsschnitt aufgenommen und in den Folgewellen erneut interviewt wurden. Am Deutschen Alterssurvey haben bislang mehr als 20000 Studienteilnehmende mit bis zu sechs Messzeitpunkten teilgenommen. Nähere Informationen zum Stichprobendesign enthält Kapitel 2 Design, Inhalte und Methodik des Deutschen Alterssurveys. Einschränkend muss darauf hingewiesen werden, dass im Deutschen Alterssurvey nahezu keine Heimbewohnerinnen und -bewohner enthalten sind, da Untersuchungsteilnehmer nach einem Umzug ins Heim überwiegend nicht mehr befragt werden können. Darüber hinaus erwies sich bei insgesamt geringer Selektivität der Panelmortalität insbesondere das Bildungsniveau als ein relevanter Einflussfaktor auf die Bleibewahrscheinlichkeit in der Studie (vgl. Kapitel 2 Design, Inhalte und Methodik des Deutschen Alterssurveys in diesem Band). Da wir für Heimbewohnerinnen und -bewohner und Niedriggebildete ein erhöhtes Risiko sozialer Isolation und Einsamkeit erwarten, sind diese beiden Selektivitäten der Panelmortalität bei der Interpretation der Ergebnisse zu berücksichtigen. In die vorliegende Untersuchung sind alle Studienteilnehmerinnen und -teilnehmer einbezogen, für die mindestens

1 Grundlage der international gebräuchlichen Einsamkeitsskala sind drei negativ und drei positiv formulierte Aussagen, zu denen jeweils anzugeben ist, ob diese auf einen selbst „genau“, „eher", „eher nicht" oder "gar nicht“ zutreffen (4-stufige Antwortskala). Die Items lauten: „Ich vermisse Leute, bei denen ich mich eine Beobachtung des Isolations- beziehungsweise Einsamkeitsrisikos vorliegt und die prinzipiell wiederholt befragt werden konnten. Um den Einfluss selektiver Panelmortalität zu verringern, wurden auch jene Studienteilnehmerinnen und -teilnehmer einbezogen, die nur an einer Befragungswelle des Deutschen Alterssurveys teilgenommen haben. Die Untersuchung des Risikos sozialer Isolation im Alternsverlauf stützt sich damit auf 39186 Messungen bei 19969 Personen. Für die Untersuchung des Einsamkeitsrisikos liegen mit 31532 Messungen bei 16151 Personen etwas geringere Fallzahlen vor, da die Einsamkeitsindikatoren im zusätzlichen Selbstausfüller-Fragenbogen erhoben wurden, den nicht alle Interviewten ausgefüllt haben.

\section{Indikatoren}

In unserer Untersuchung messen wir soziale Isolation anhand der Größe des persönlichen Netzwerks, bestehend aus den Personen, die den Individuen nach eigener Aussage persönlich wichtig sind und mit denen sie regelmäßig Kontakt haben. Nur wenn sie niemanden oder maximal eine Person nennen können, auf die das zutrifft, werten wir dies als soziale Isolation. Dabei zählen wir Haushaltsmitglieder des Individuums grundsätzlich zu seinem Netzwerk. Soziale Isolation wird für die Analysen also als dichotome Variable mit den beiden Ausprägungen 1 (isoliert) und 0 (nicht isoliert) verwendet. Zur Bestimmung der Einsamkeit stützen wir uns auf die im Deutschen Alterssurvey verwendete Einsamkeitsskala in der Kurzform von de Jong Gierveld und van Tilburg (2006) mit einem Mittelwert aus sechs Items. ${ }^{1}$ Die Skalenausprägungen können vom Wert 1 (gar nicht einsam)

wohl fühle“, „Ich fühle mich häufig im Stich gelassen“, „Ich vermisse Geborgenheit und Wärme“, „Es gibt genug Menschen, die mir helfen würden, wenn ich Probleme habe“, „Ich kenne viele Menschen, auf die ich mich wirklich verlassen kann“, „Es gibt genügend Menschen, mit denen ich mich eng verbunden füh- 
bis 4 (sehr einsam) reichen. Zu den Einsamen zählen wir Personen mit einem Skalenwert, der größer als 2,5 ist, sich also in der oberen Hälfte des möglichen Wertebereichs der Skala befindet. Mit dieser Aufteilung der Skalenwerte erfassen wir Einsamkeit als dichotome Variable mit den beiden Ausprägungen 1 (einsam) und 0 (nicht einsam). Zur Interpretation der folgenden Ergebnisse muss deshalb beachtet werden, dass wir sehr strikte Kriterien für die Klassifizierung benutzen. Menschen, die wir mit unserer Methode als sozial isoliert einstufen, haben einen sehr deutlichen Mangel im Austausch mit anderen. Personen, für die wir das Kriterium, einsam zu sein, als erfüllt ansehen, verspüren eine tiefe Einsamkeit.

Zentrale erklärende Variablen sind das Alter zum jeweiligen Befragungszeitpunkt und das Geschlecht. Zur Bestimmung des linearen Alterseffekts verwenden wir das Alter in Jahren, um mögliche nicht-lineare Alterseinflüsse zu erfassen, verwenden wir ergänzend das quadrierte Alter. Um feststellen zu können, ob Alters- und Geschlechterunterschiede in den Risikoverläufen für soziale Isolation und Einsamkeit einem gesellschaftlichen Wandel unterliegen, testen wir auch den Unterschied zwischen Geburtskohorten. Für die Untersuchung von Kohortenunterschieden beschränken wir uns auf Geburtsjahrgänge, für die im Deutschen Alterssurvey mehrere Messzeitpunkte über eine größere Altersspanne vorliegen, und bilden die folgenden drei Kohorten: Geburtsjahrgänge 1930-1939, 1940-1949, 1950-1959.

\section{Statistisches Analyseverfahren}

Für die Alternsverläufe schätzen wir Random-Effects-Panelregressionen. Dieses Verfahren fokussiert auf die individuellen Entwicklungen über die Messzeitpunkte und analysiert damit Alternsverläufe des Risikos sozialer Isolation und Einsamkeit.
Die Schätzungen erfolgen mittels logistischer Regressionsverfahren, da die abhängigen Variablen dichotom sind (isoliert vs. nicht isoliert; einsam vs. nicht einsam). Zur Bestimmung möglicher Unterschiede zwischen Frauen und Männern wird das Geschlecht sowohl als eigenständiger Prädiktor als auch als eine den Alternsverlauf moderierende Variable aufgenommen (Interaktion von Alter und Geschlecht). Die zusätzlichen Modelle zur Analyse möglicher Kohorteneffekte basieren auf den 1930 bis 1959 Geborenen und enthalten die drei 10-Jahres-Kohorten als Prädiktoren. Bei allen Modellierungen wurde zudem die regionale Zugehörigkeit zu den alten oder neuen Bundesländern als Kontrollvariable aufgenommen, um Ergebnisverzerrungen durch den überproportionalen Einbezug von in Ostdeutschland Wohnenden bei der Stichprobenziehung zu verhindern.

Sofern die im jeweiligen Regressionsmodell einbezogenen Prädiktoren einen statistisch signifikanten Einfluss $(p<0,05)$ auf das Isolations- oder Einsamkeitsrisiko haben, werden auf der Grundlage der Modellergebnisse altersjahrspezifische Wahrscheinlichkeitswerte für das Vorhandensein von Isolation oder Einsamkeit der Frauen und Männer berechnet. Diese lassen sich grafisch darstellen mit einem Wertebereich von null bis 100 Prozent. Die in den Abbildungen dargestellten Alternsverläufe kennzeichnen die sich aus den Alterseffekten vorhergesagten Wahrscheinlichkeiten von sozialer Isolation oder Einsamkeit. Sie beziffern das längsschnittliche Risiko, mit dem Älterwerden sozial isoliert oder einsam zu sein. In allen statistischen Untersuchungen wurden Unterschiede sowohl im Niveau bei verschiedenen Altersjahren als auch im linearen und quadratischen Alternsverlauf der Wahrscheinlichkeit von sozialer Isolation und Einsamkeit auf statistische Signifikanz geprüft.

Zunächst untersuchen wir, wie sich diese Risiken über die zweite Lebenshälfte für Frauen und Männer entwickeln und stellen hierzu den Risiko-

le“. Die Antworten auf die drei negativen Items werden umgepolt, so dass bei allen Items hohe Werte auf ein hohes Ausmaß von Einsamkeit verweisen. 
verlauf im Altersbereich von 40 bis 90 Jahren dar. Im nächsten Analyseschritt widmen wir uns den geschlechtsspezifischen Verläufen im Vergleich der
Geburtskohorten, die zwischen 1930 bis 1939, zwischen 1940 bis 1949 und zwischen 1950 bis 1959 geboren sind.

\subsection{Ergebnisse}

\subsubsection{Soziale Isolation im Verlauf der zweiten Lebenshälfte}

Das Risiko sozialer Isolation steigt mit dem Älterwerden. Bis etwa zum 75. Lebensjahr haben Männer ein höheres Isolationsrisiko als Frauen.

Im Folgenden berichten wir, wie sich das individuelle Älterwerden auf das Risiko, sozial isoliert und/oder einsam zu sein, bei Frauen und Männern auswirkt. Insgesamt zeigt sich ein über die zweite Lebenshälfte ansteigendes Risiko sozialer Isolation mit einem signifikanten Geschlechterunterschied im Alternsverlauf. Wie in Abbildung 5-1 dargestellt, steigt das Risiko sozial isoliert zu sein, mit dem Älterwerden an. Im Alter von 40 Jahren liegt dieses Risiko für beide Geschlechter bei etwa vier Prozent. Im Verlauf des weiteren Lebens steigt das Isolationsrisiko im Durchschnitt auf etwa 22 Prozent im Alter von 90 Jahren an.

Für Frauen und Männer gestalten sich diese Verläufe jedoch unterschiedlich. Männer weisen im Alter von 40 Jahren ein Isolationsrisiko von etwa fünf Prozent auf und dieses Risiko steigt ste-

Abbildung 5-1 Risiko sozialer Isolation bei Frauen und Männern im Alternsverlauf

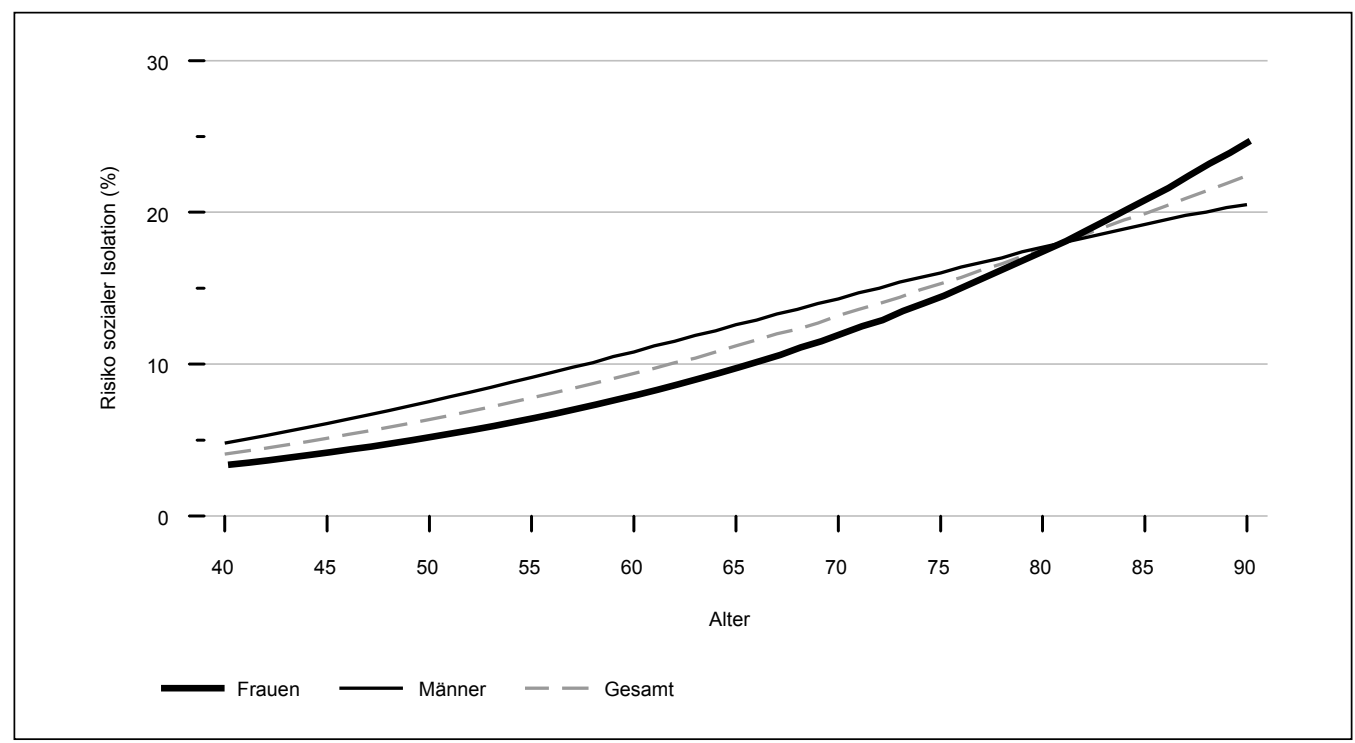

Quelle: DEAS 1996-2017, n = 19969 Studienteilnehmende mit insgesamt n = 39186 Beobachtungen; modellbasierte Schätzungen kontrolliert für Region; der Geschlechterunterschied im Alternsverlauf ist signifikant.

Die Wahrscheinlichkeit, im Alter von 65 Jahren sozial isoliert zu sein, beträgt für Frauen ca. zehn Prozent und für Männer ca. 13 Prozent. 
tig auf etwa 20 Prozent im Alter von 90 Jahren an. Das Risiko, isoliert zu sein, liegt bei Frauen Anfang 40 bei etwa drei Prozent. Dieses Risiko steigt zunächst weniger stark an als bei den Männern, erfährt aber im späteren Lebensverlauf etwa ab Ende 60 einen beschleunigten Zuwachs. Dementsprechend unterscheidet sich das Risiko, sozial isoliert zu sein, im mittleren Lebensalter und frühen hohen Alter zwischen Männern und Frauen. Im Alter von 65 Jahren haben Frauen beispielsweise ein Risiko von etwa zehn Prozent während Männer ein Risiko von etwa 13 Prozent aufweisen. Im hohen Alter gleicht sich das Risiko wieder an. Die Geschlechter unterscheiden sich ab dem 75 . Lebensjahr nicht mehr signifikant.

Der Alternsverlauf des Risikos, sozial isoliert zu sein, hat sich gewandelt. Bei später geborenen Geburtskohorten steigt das Isolationsrisiko mit dem Älterwerden nicht mehr auf einen so hohen Wert an wie bei den früher geborenen Jahrgängen.
Es zeigten sich signifikante Unterschiede in den geschätzten Risikoverläufen sozialer Isolation zwischen den zusammengefassten Geburtsjahrgängen von 1930-1939, 1940-1949 und 1950-1959 (Abbildung 5-2). Im Untersuchungszeitraum haben sich die altersspezifischen Risiken des Auftretens sozialer Isolation zwischen den drei betrachteten Kohorten eher verringert. Diese Verringerung wird sich aller Voraussicht nach insbesondere nach dem Erreichen des 60. Lebensjahres besonders bemerkbar machen. Während die Schätzung des allgemeinen Verlaufs unter Einschluss aller Geburtsjahrgänge (1911 bis 1974) einen stetigen Zuwachs des Isolationsrisikos über das Lebensalter anzeigt, so ergibt die vergleichende Betrachtung der drei Geburtskohorten 1930 bis 1939, 1940 bis 1949 und 1950 bis 1959 ein anderes Bild. Bei den zwischen 1930 und 1939 Geborenen steigt das Isolationsrisiko bis zum Alter Mitte 70 und stabilisiert sich dann bis in höchste Alter bei etwa 16 Prozent. Für die nachfolgenden Geburtskohorten zeigt sich die Abflachung

\section{Abbildung 5-2 Risiko sozialer Isolation nach Kohorten im Alternsverlauf}

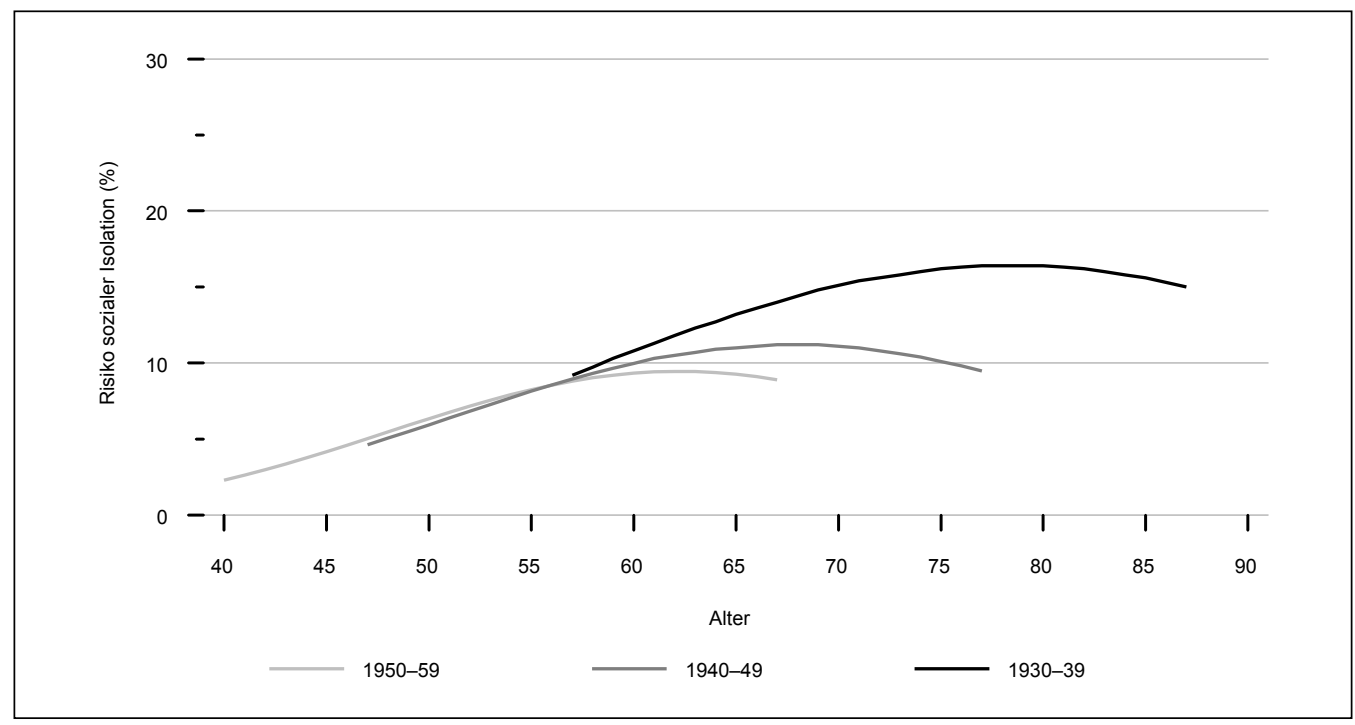

Quelle: DEAS 1996-2017, n = 13899 Studienteilnehmende mit insgesamt n = 29069 Beobachtungen; modellbasierte Schätzungen kontrolliert für Region und Geschlecht; die Kohortenunterschiede sind signifikant.

Die Wahrscheinlichkeit, im Alter von 65 Jahren sozial isoliert zu sein, beträgt für die 1930-1939 Geborenen ca. 13 Prozent und für die 1950-1959 Geborenen ca. neun Prozent. 
des Isolationsrisikos in noch früherem Alter und auf einem tieferen Niveau. Bei den Geburtsjahrgängen zwischen 1940 und 1949 endet der alternsbezogene Anstieg des Isolationsrisikos schon etwa beim 70. Lebensjahr und das Risiko erreicht nur noch ein maximales Niveau von etwa elf Prozent. Für die Jahrgänge 1950 bis 1959 endet der Anstieg schon beim Alter von 65 Jahren mit einem maximalen Isolationsrisiko von nur noch neun Prozent.

Für alle Kohorten zeigen sich ähnliche Geschlechterunterschiede in den Alternsverläufen des Isolationsrisikos.

Wie in Abbildung 5-3 dargestellt, werden die Geschlechterunterschiede im Vergleich über die Geburtskohorten weder kleiner noch größer; sie entsprechen in etwa den Geschlechterunterschieden, die in der Analyse aller Geburtsjahrgänge sichtbar wurde (vgl. Abbildung 5-1).

\subsubsection{Einsamkeit im Verlauf der zweiten Lebenshälfte}

Der Verlauf des Einsamkeitsrisikos in der zweiten Lebenshälfte ist u-förmig. Männer haben ein höheres Risiko im mittleren Lebensalter, Frauen haben ein höheres Risiko im hohen Alter.

Der Verlauf im Einsamkeitsrisiko von Frauen und Männern unterscheidet sich deutlich von der Entwicklung des Isolationsrisikos. Das Einsamkeitsrisiko ist zu Beginn der zweiten Lebenshälfte bei beiden Geschlechtern höher als das Isolationsrisiko, nimmt dann im Alternsverlauf bei Frauen und Männern zunächst ab, um im höheren Alter wieder anzusteigen (Abbildung 5-4). Während das Isolationsrisiko mit steigendem Alter relativ kontinuierlich zunimmt, zeigt sich bei der Einsamkeit ein signifikanter kurvilinearer Verlauf. 40-Jährige haben ein Einsamkeitsrisiko von elf Prozent. Diese Wahrscheinlichkeit verringert sich zunächst über die nächsten 30 Lebensjahre. Das Einsamkeitsrisiko im Alter von 70 Jahren liegt bei acht Prozent.
Danach steigt es wieder an. 90-jährige Menschen haben - wie die 40-Jährigen - ein Einsamkeitsrisiko von elf Prozent.

Im Alternsverlauf des Einsamkeitsrisikos gibt es einen signifikanten Geschlechterunterschied. Männer haben im mittleren Erwachsenenalter ein höheres Einsamkeitsrisiko als Frauen. Der Geschlechterunterschied nimmt mit steigendem Alter jedoch ab und dreht sich im Verlauf des Rentenalters um, so dass im hohen Alter mehr Frauen als Männer einsam sind. Im Alter von 40 Jahren beträgt das Risiko, einsam zu sein für Männer etwa 13 Prozent und für Frauen ca. zehn Prozent. Im weiteren Alternsverlauf verringert sich dieser Unterschied und gleicht sich etwa ab dem 62. Lebensjahr aus. Mit 65 Jahren haben Frauen und Männer ein nahezu gleich hohes Einsamkeitsrisiko von knapp acht Prozent. Danach steigt das Einsamkeitsrisiko mit dem Älterwerden stärker bei Frauen als bei Männern. Mit 90 Jahren haben Frauen ein Risiko von 14 Prozent, einsam zu sein. Bei Männern in diesem Alter beträgt das Einsamkeitsrisiko hingegen nur neun Prozent.

Bei später geborenen Geburtskohorten ist der Verlauf des Einsamkeitsrisikos weniger vom Lebensalter abhängig.

Es zeigen sich ebenfalls signifikante Unterschiede in den geschätzten Risikoverläufen zwischen den Geburtskohorten von 1930 bis 1939, 1940 bis 1949 und 1950 bis 1959 (Abbildung 5-5). Im Untersuchungszeitraum haben sich die altersspezifischen Risiken des Auftretens von Einsamkeit zwischen den drei betrachteten Kohorten tendenziell verringert. Die Veränderungen über die Geburtskohorten sind allerdings schwerer zu erkennen als die Kohortenunterschiede in der sozialen Isolation. Abbildung 5-5 zeigt, dass das Einsamkeitsniveau im Alter zwischen 40 und 50 Jahren bei der jüngsten der drei betrachteten Kohorten gesunken ist und sich im Alternsverlauf bis Mitte 60 wenig verändert. Im Vergleich zur ältesten betrachteten Kohorte deutet sich eine Verlagerung des altersbezogenen Anstiegs des Einsamkeitsrisi- 
Abbildung 5-3 Risiko sozialer Isolation bei Frauen und Männern nach Kohorten im Alternsverlauf

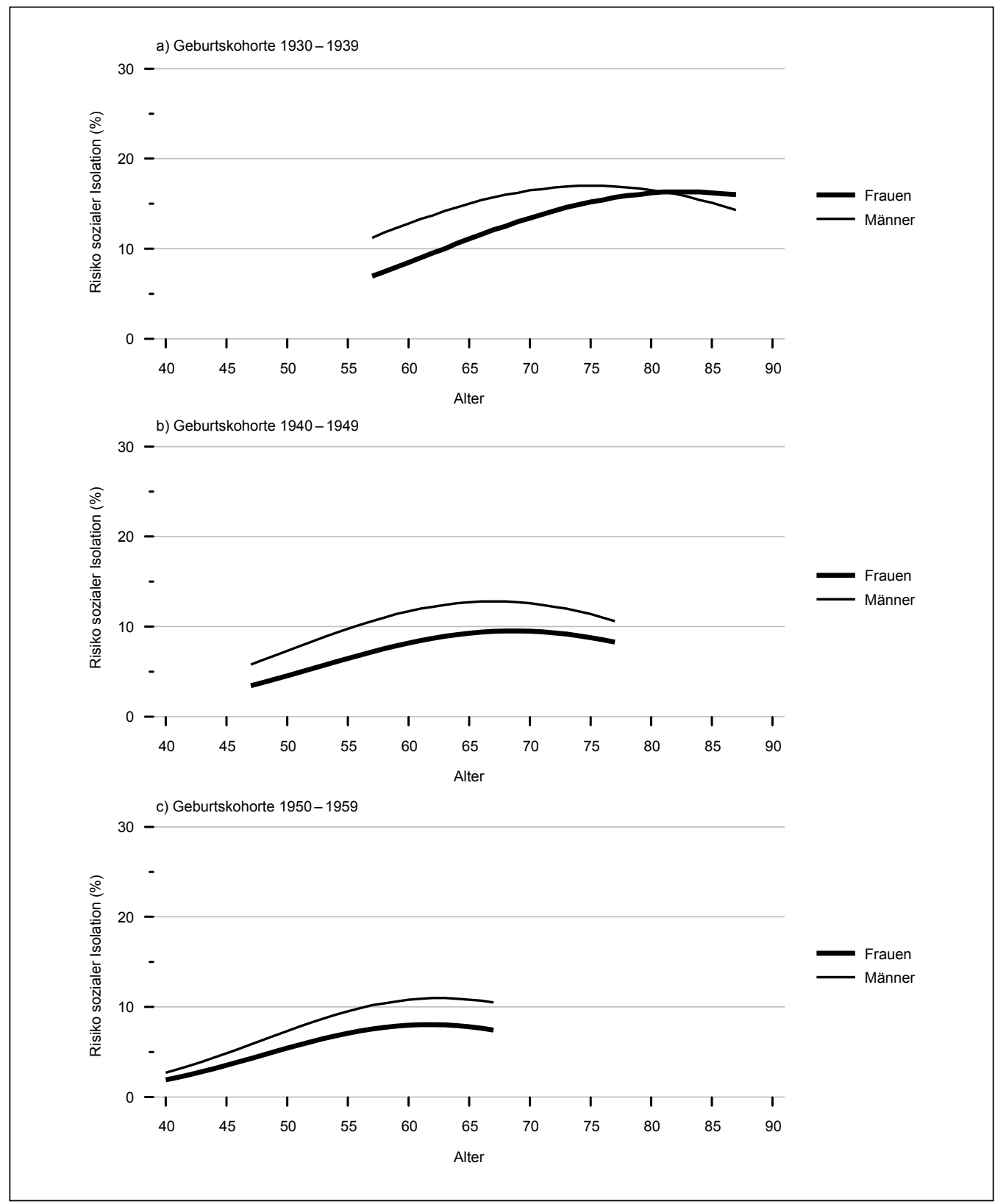

Quelle: DEAS 1996-2017, n = 13899 Studienteilnehmende mit insgesamt $n=29069$ Beobachtungen; modellbasierte Schätzungen kontrolliert für Region; kein signifikanter Interaktionseffekt zwischen Geschlecht und Geburtskohorte, die Geschlechterunterschiede haben sich zwischen den Geburtskohorten nicht verändert.

Die Wahrscheinlichkeit, im Alter von 65 Jahren sozial isoliert zu sein, beträgt für 1950-1959 geborene Männer ca. elf Prozent, für Frauen ca. acht Prozent. 
Abbildung 5-4 Einsamkeitsrisiko bei Frauen und Männern im Alternsverlauf

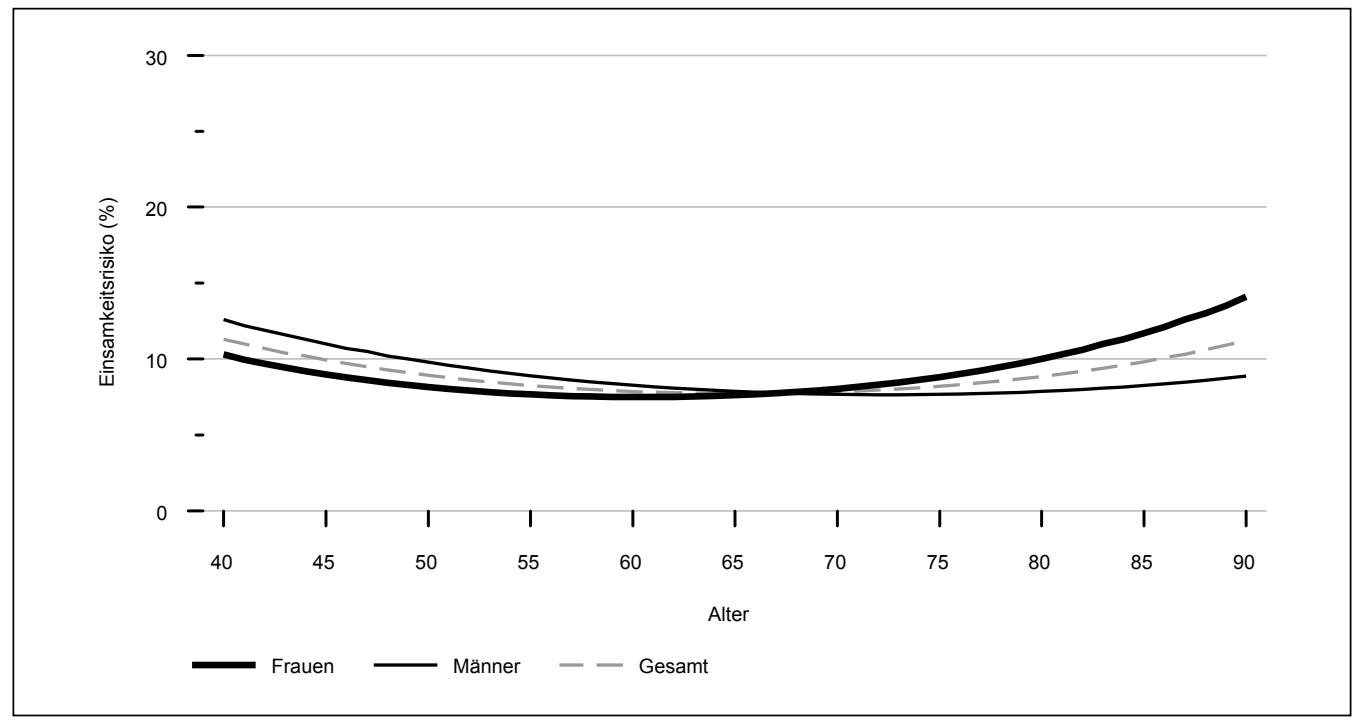

Quelle: DEAS 1996-2017, n = 16151 Studienteilnehmende mit insgesamt $n=31532$ Beobachtungen; modellbasierte Schätzungen kontrolliert für Region; der Geschlechtereffekt ist signifikant.

Die Wahrscheinlichkeit, im Alter von 90 Jahren einsam zu sein, beträgt für Frauen ca. 14 Prozent und für Männer ca. neun Prozent.

\section{Abbildung 5-5 Einsamkeitsrisiko nach Kohorten im Alternsverlauf}

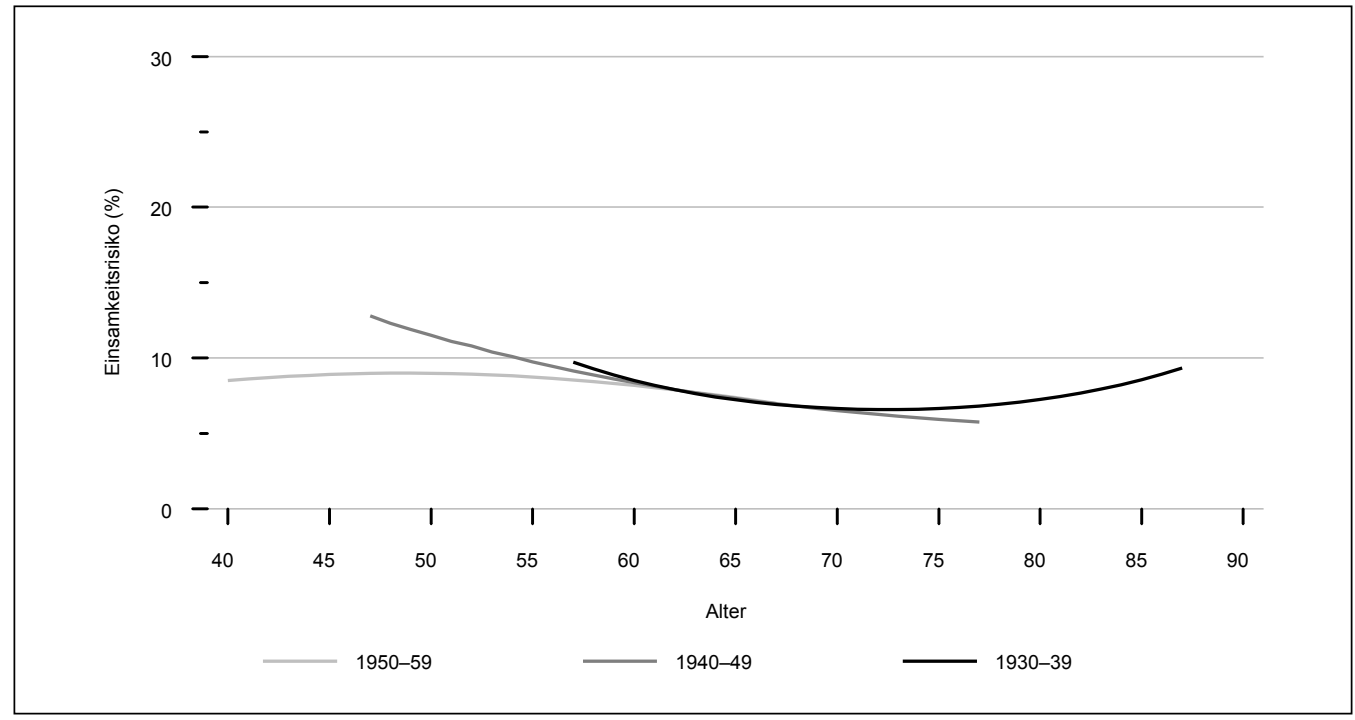

Quelle: DEAS 1996-2017, n = 11405 Studienteilnehmende mit insgesamt n = 23397 Beobachtungen; modellbasierte Schätzungen kontrolliert für Region und Geschlecht; der Kohorteneffekt ist signifikant.

Alle drei Geburtsjahrgangsgruppen haben im Alter von 65 Jahren ein Einsamkeitsrisiko von ca. sieben Prozent. 
kos in höhere Altersjahre an. Mit anderen Worten: Das Einsamkeitsrisiko ist in den später geborenen Jahrgängen weniger stark mit dem Älterwerden verknüpft als in den früher geborenen Geburtskohorten.

In allen betrachteten Kohorten zeigen sich ähnliche Geschlechterunterschiede in den Alternsverläufen des Einsamkeitsrisikos.

Wie beschrieben gibt es Unterschiede in den Alternsverläufen des Einsamkeitsrisikos sowohl zwischen Frauen und Männern als auch zwischen Geburtsjahrgängen. Deshalb wurde in einem nächsten Untersuchungsschritt betrachtet, ob oder inwieweit sich die gleichen Geschlechterunterschiede in den verschiedenen Geburtskohorten wiederfinden lassen. $\mathrm{Zu}$ diesem Zweck wurde untersucht, ob sich statistisch eine Interaktion zwischen den Faktoren Geschlecht und Kohorte in Bezug auf die Alternsverläufe nachweisen ließ.

\subsection{Diskussion}

\section{Zusammenfassung}

Unsere längsschnittlichen Analysen der individuellen Entwicklungen zeigen, dass das Risiko sozialer Isolation zu Beginn der zweiten Lebenshälfte noch sehr niedrig ist, aber mit dem Älterwerden steigt und sich bei Frauen und Männern unterschiedlich entwickelt. Männer im Alter von 40 Jahren haben ein etwas höheres Isolationsrisiko als Frauen. Zudem steigt dieses im Verlauf der zweiten Lebenshälfte relativ gleichmäßig bis ins hohe Alter an. Bei Frauen ist das Isolationsrisiko anfangs niedriger und nimmt zunächst auch weniger stark zu. Im Rentenalter erhöht sich das Isolationsrisiko der Frauen jedoch schneller als das der Männer, so dass sie im hohen Alter (80 Jahre und älter) ähnlich häufig oder sogar etwas häufiger dem Risiko ausgesetzt sind, sozial isoliert zu sein. Zuvor haben
Die statistische Analyse ergab keinen signifikanten Interaktionseffekt von Geschlecht und Geburtskohorte, das heißt die Geschlechterunterschiede der Verläufe des Einsamkeitsrisikos haben sich nicht verändert. Berücksichtigt man den Interaktionsterm dennoch in der modellbasierten Schätzung der Alternsverläufe des Einsamkeitsrisikos, ergibt sich folgendes Bild (Abbildung 5-6). In der jüngsten der drei Kohorten zeigt sich das bereits erwähnte höhere Einsamkeitsrisiko der Männer zwischen dem 40. und 60. Lebensjahr, die mittlere Kohorte weist geringe Geschlechterdifferenzen auf und in der ältesten der drei Kohorten zeichnet sich der stärkere Risikoanstieg bei den Frauen im hohen Alter ab. Dass der Geschlechterunterschied im hohen Alter in Abbildung 5-6 nicht so deutlich hervortritt wie in der Gesamtschau in Abbildung 5-4 dürfte der Tatsache geschuldet sein, dass in Abbildung 5-4 alle Untersuchungsteilnehmerinnen und -teilnehmer, auch die vor $1930 \mathrm{Ge}$ borenen einbezogen sind, die besonders die Befunde zum höheren Alter beeinflussen.

Frauen allerdings mehr als drei Lebensjahrzehnte lang von Anfang 40 bis Mitte 70 ein geringeres Isolationsrisiko als Männer. In der Abfolge der Geburtskohorten verändert sich die Entwicklung des Isolationsrisikos mit dem Älterwerden. In später geborenen Geburtskohorten wird das Isolationsrisiko im höchsten Lebensalter vermutlich nicht mehr weiter ansteigen und unter dem früher geborener Geburtsjahrgänge bleiben. Die Geschlechterunterschiede in den Alternsverläufen sozialer Isolation sind weitgehend stabil geblieben und haben sich in der Abfolge der untersuchten Kohorten weder verringert noch vergrößert.

Das Einsamkeitsrisiko weist im Alternsverlauf von 40 bis 90 Jahren einen leicht u-förmigen Verlauf auf. Im Beobachtungszeitraum ist es am höchsten im Alter von 40 Jahren und liegt in diesem Alter deutlich über dem Isolationsrisiko. Im 
Abbildung 5-6 Einsamkeitsrisiko bei Frauen und Männern nach Kohorten im Alternsverlauf

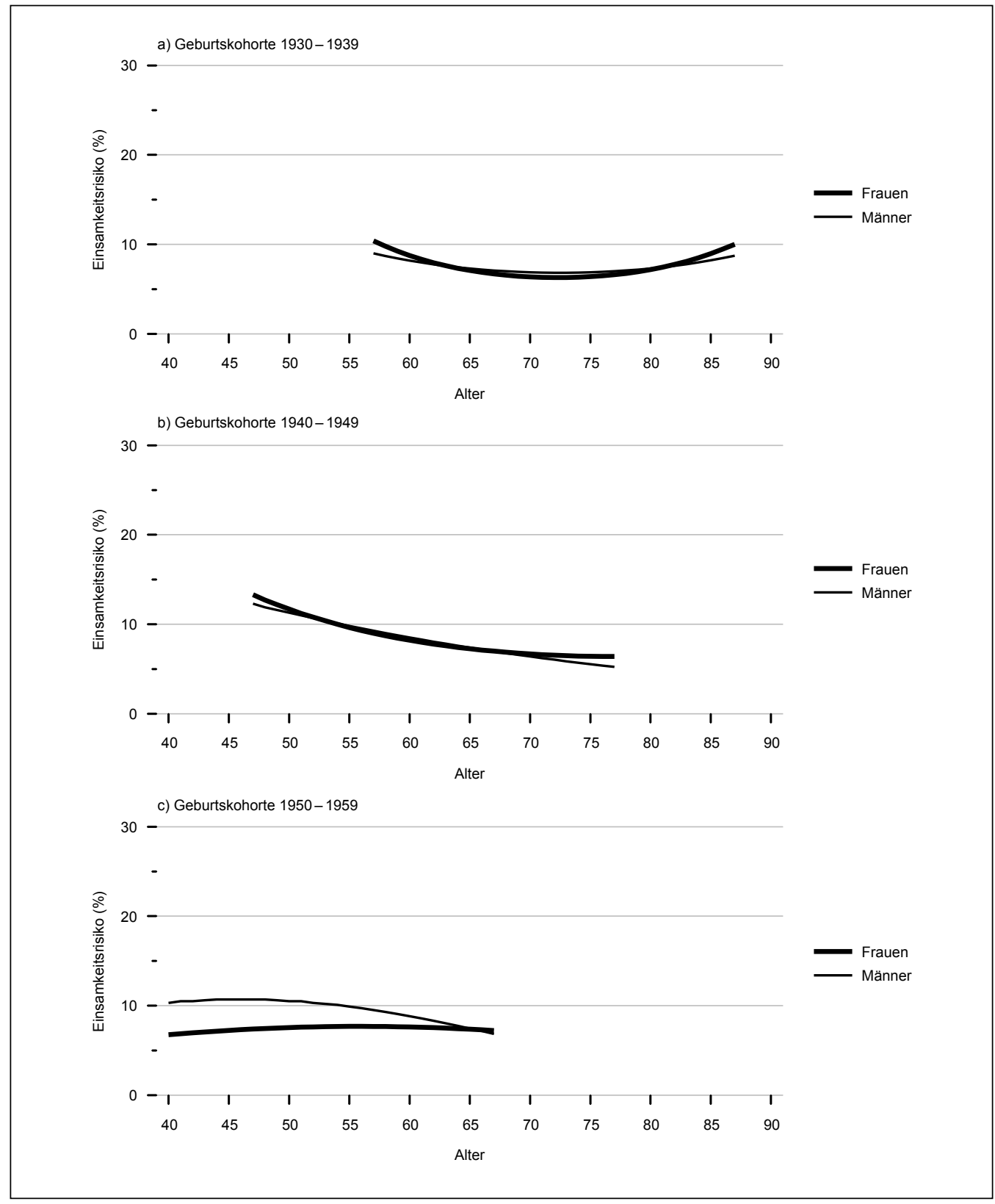

Quelle: DEAS 1996-2017, n = 11405 Studienteilnehmende mit insgesamt n = 23397 Beobachtungen; modellbasierte Schätzungen kontrolliert für Region; kein signifikanter Interaktionseffekt zwischen Geschlecht und Geburtskohorte, die Geschlechterunterschiede haben sich zwischen den Geburtskohorten nicht verändert.

Die Wahrscheinlichkeit, im Alter von 6o Jahren einsam zu sein, beträgt für 1950-1959 geborene Männer ca. neun Prozent und für Frauen ca. acht Prozent. 
weiteren Alternsverlauf verringert es sich bis etwa zum 70. Lebensjahr und steigt dann langsam wieder an. Wenn Menschen 90 Jahre alt sind, haben sie in etwa das gleiche Einsamkeitsrisiko wie mit 40 Jahren. Die Form des Alternsverlaufs im Einsamkeitsrisiko unterscheidet sich zwischen den Geschlechtern und Geburtskohorten. Während Männer von 40 bis etwa 60 Jahren ein vergleichsweise höheres Einsamkeitsrisiko erleben, haben Frauen ein größeres Einsamkeitsrisiko als Männer, nachdem sie etwa das 70. Lebensjahr erreicht haben. Bei später geborenen Geburtsjahrgängen zeigt sich ein etwas niedrigeres Einsamkeitsrisiko zu Beginn der zweiten Lebenshälfte und eine Abflachung des u-förmigen Verlaufs. Aller Voraussicht nach wird sich der Anstieg des Einsamkeitsrisikos im hohen Alter abschwächen. Die Unterschiede in den Alternsverläufen zwischen den Geschlechtern sind von diesem sozialen Wandel nicht betroffen.

\section{Interpretation}

Wenn man sich die Verläufe über die gesamte zweite Lebensspanne genauer ansieht, kristallisieren sich bestimmte Lebensphasen heraus, in denen älterwerdende Menschen besonders verwundbar zu sein scheinen. Das Risiko sozial isoliert zu sein, erreicht im hohen Erwachsenenalter die höchsten Werte. Wie aus der Forschung bekannt ist, verringert sich die Größe der sozialen Netzwerke kontinuierlich über die zweite Lebenshälfte (Wrzus, Hanel, Wagner, \& Neyer 2013). Wie unsere Analysen unseres Wissens nach erstmalig zeigen, korrespondiert diese allgemeine Entwicklung mit einer in weiten Teilen linearen Zunahme des individuellen Risikos sozialer Isolation im Verlauf der zweiten Lebenshälfte. Die Verringerung der Netzwerkgröße führt demnach häufig dazu, dass die betroffenen Menschen niemanden mehr oder nur noch eine einzige Person haben, mit der sie regelmäßig Kontakt haben und die für sie wichtig ist. Diese negative Entwicklung im Alter kann unter anderem bedingt sein durch den Verlust von Netzwerkpersonen durch Mortalität oder Wegzug, aber auch durch schwindende gesundheitliche Ressourcen, die bedeutsame soziale Kontakte erschweren (Huxhold, Fiori, \& Windsor 2013; Huxhold \& Fiori 2018).

Frauen und Männer unterscheiden sich in ihren Risikolagen im Lebensverlauf. Im mittleren Bereich der beobachteten Lebensspanne etwa ab dem Alter von 40 Jahren bis hin zum 75 . Lebensjahr haben Frauen ein geringeres Risiko als Männer sozial isoliert zu sein. Dieser Vorteil hat möglicherweise mit einem höheren Investment von Frauen in ihre sozialen Netzwerke, insbesondere in ihre Freundschaften, zu tun. Erst im hohen Erwachsenenalter nach dem 75 . Lebensjahr scheinen die verstärkt bei Frauen vorkommenden funktionalen Einschränkungen (siehe Kapitel 3 Funktionale und subjektive Gesundheit in diesem Band) und das vergleichsweise hohe weibliche Verwitwungsrisiko den sozialen Integrationsvorteil von Frauen aufzuheben.

Insgesamt lassen die Ergebnisse jedoch erkennen, dass selbst im Alter von 90 Jahren rund 80 Prozent der in einem Privathaushalt lebenden Menschen in Deutschland vermutlich nicht isoliert sein werden. Die Mehrheit der Hochbetagten pflegt mindestens zwei Beziehungen zu anderen ihnen wichtigen Personen mit regelmäßigem Austausch. Das mit 20 Prozent dennoch relativ hohe Risiko sozialer Isolation im hohen Alter schlägt sich nicht im besonderen Maße in einem proportional gestiegenen Einsamkeitsrisiko nieder.

Obwohl unsere Untersuchungen einen leichten Anstieg des Einsamkeitsrisikos ab etwa dem 70. Lebensjahr verzeichnen, bewegt sich das Einsamkeitsrisiko beim Erreichen des 90. Lebensjahres auf dem gleichen Niveau wie das Risiko im Alter von 40 Jahren. Dieses Phänomen lässt sich möglicherweise dadurch erklären, dass sich mit dem Älterwerden die individuellen Bedürfnisse in Bezug auf soziale Kontakte ändern und sich Menschen im hohen Alter insbesondere auf den emotionalen Austausch konzentrieren (Carstensen, Isaacowitz, \& Charles 1999). Dieses emotionale Bedürfnis kann möglicherweise auch von sehr wenigen, vielleicht sogar von nur einer Person befriedigt werden. 
Ein starkes Bedürfnis nach sozialer Integration könnte hingegen erklären, warum unsere Analysen ein vergleichsweise hohes Risiko für Einsamkeit zwischen dem 40. und dem 50. Lebensjahr verzeichnen. In der Literatur wird häufig angenommen, dass gerade diese Lebensphase gekennzeichnet ist durch intensive Prozesse der Selbstbewertung, in denen die persönlichen Errungenschaften in allen Lebensbereichen, auch im Bereich der sozialen Beziehungen, sowohl mit den eigenen Erwartungen als auch den vorherrschenden sozialen Normen verglichen werden (Freund \& Ritter 2009). Deshalb mögen negative kritische Lebensereignisse - wie die Auflösung einer Partnerschaft oder ein Krankheitsereignis - zu diesem Zeitpunkt besonders starke Auswirkungen auf das Risiko haben einsam zu werden, denn die eigenen und sozialen Erwartungen sind in dieser Lebensphase auf Grund der oben genannten Vergleichsprozesse besonders präsent.

Die Einsamkeitsrisikoverläufe von Frauen und Männern sind unterschiedlich und spiegeln in gewisser Weise die Geschlechterunterschiede in den Verläufen des Isolationsrisikos wider. Im Alter von 40 bis 60 Jahren haben Männer ein höheres Risiko als Frauen einsam zu sein, vermutlich weil Frauen in dieser Zeit ihre sozialen Beziehungen intensiver pflegen. Mit steigendem Alter haben Frauen eine höhere Wahrscheinlichkeit als Männer, von Verwitwung und Mobilitätseinschränkungen betroffen zu sein. Diese geschlechtsspezifischen Risikofaktoren führen vermutlich dazu, dass das Einsamkeitsrisiko ab Anfang 70 für Frauen stärker ansteigt als für Männer.

Angesichts der öffentlichen Debatte um eine vermeintliche „Epidemie der Einsamkeit“ ist es wichtig, ein Ergebnis besonders hervorzuheben: In unseren Analysen der individuellen Verläufe der Risiken für soziale Isolation und Einsamkeit von Männern und Frauen ergaben sich Anzeichen für eine Abschwächung der altersbezogenen Zunahme des Einsamkeits- und Isolationsrisikos und für eine Stagnation des Anteils der im Alter isoliert lebenden und/oder sich einsam fühlenden Menschen. Anders ausgedrückt: Wenn sich die gesell- schaftlichen Gegebenheiten in der nächsten Dekade nicht radikal ändern, werden die dann 70- bis 90-Jährigen nicht häufiger isoliert oder einsam sein als die heute 70- bis 90-Jährigen. Wahrscheinlich werden die oft vermuteten negativen Auswirkungen auf die sozialen Beziehungen der häufig berichteten Trends zu einer vermehrten Kinderlosigkeit, erhöhten Trennungsraten und größeren räumlichen Wohnentfernungen zwischen Eltern und ihren erwachsenen Kindern ausgeglichen durch eine größere Wichtigkeit von Freundschaften und verbesserte Kommunikationsmöglichkeiten. Auswertungen des Deutschen Alterssurveys (DEAS) weisen beispielsweise auf eine in den letzten Jahrzehnten steigende Bedeutung von Freundinnen und Freunden im persönlichen Netzwerk hin (Huxhold et al. 2010). Der gesellschaftliche Wandel in den Familienbeziehungen über die verschiedenen Geburtskohorten hinweg und die Entwicklung zu mehr Freiheit in der Gestaltung der sozialen Beziehungen (Allan 2008) führen demnach zwar zu einer Veränderung in der Zusammensetzung der persönlichen Netzwerke, aber vermutlich nicht zu einer problematischen Verringerung der persönlichen Netzwerkgrößen oder einer Zunahme an Einsamkeit und sozialer Isolation.

\section{Politische Implikationen}

Die Analysen, die in diesem Kapitel präsentiert wurden, können zwei wichtige Erkenntnisse zu der öffentlichen Debatte um eine sich vermeintlich ausbreitende Einsamkeit („Epidemie“) beitragen. Soziale Isolation und Einsamkeit sind keine unausweichlichen Schicksale des Alter(n)s. Die überwiegende Mehrheit aller Menschen wird auch im hohen Alter weder sozial isoliert noch einsam sein. Außerdem hat der Anteil an Frauen und Männern, die soziale Isolation und Einsamkeit im Alter erleben, nicht zugenommen und wird es aller Voraussicht nach auch in der nächsten Dekade nicht. Diese Erkenntnisse sind deshalb so wichtig, weil die Erwartungen, die Menschen von ihrem eigenen Alter haben, zu selbsterfüllenden Prophezeiungen 
werden können, die den eigenen Alternsprozess beeinflussen (Levy 2009). Eine neuere Studie fand in diesem Zusammenhang heraus, dass gerade Menschen mit einem positiven Ausblick auf das eigene Älterwerden besser in der Lage sind, Freundschaften aufrechtzuerhalten und im Alter neue zu schließen (Menkin, Robles, Gruenewald, Tanner, \& Seeman 2017). Zusätzlich ergaben weitere Analysen auf Grundlage des Deutschen Alterssurveys, dass ein Teil des Trends zur wachsenden Bedeutung und Wichtigkeit von Freundschaften im höheren Erwachsenenalter mit einer Verbesserung der Vorstellungen vom Älterwerden zusammenhängt (Huxhold 2019). Eine einseitige öffentliche Debatte über Einsamkeit, die auf einer Unausweichlichkeit sozialer Isolation und Einsamkeit im Alter beharrt, hat demzufolge das Potenzial, die individuellen Vorstellungen vom Älterwerden zum Negativen zu beeinflussen, und ruft vielleicht dadurch erst das Phänomen hervor, das es eigentlich $\mathrm{zu}$ vermeiden versucht.

Dennoch ist die öffentliche Debatte um das Thema wichtig. Trotz der eher erfreulichen Gesamtergebnisse, die in diesem Kapitel präsentiert wurden, muss man bedenken, dass unsere Kriterien für soziale Isolation und Einsamkeit sehr strikt sind. Das bedeutet, dass Menschen, die gemäß unserer Klassifikation als sozial isoliert oder einsam eingestuft werden, sich in stark emotional belastenden und Beziehungsressourcen einschränkenden Situationen befinden, die mit einer ganzen Reihe von Risiken für die mentale und physische
Gesundheit einhergehen. Obwohl die überwiegende Mehrheit der Frauen und Männer weder sozial isoliert noch einsam sind, werden immer noch mehrere hunderttausend Menschen in der zweiten Lebenshälfte von sozialer Isolation und Einsamkeit betroffen sein. Angesichts der schwerwiegenden gesundheitlichen Folgen bedeutet deshalb die Bekämpfung sozialer Isolation und Einsamkeit nichts anderes als breitflächige Gesundheitsvorsorge.

Ein erster Schritt dazu könnte eine Aufklärungskampagne sein, die das soziale Stigma bekämpft, welches es vielen Menschen - Frauen wie Männern - schwer macht, über ihre Einsamkeit zu reden und sich Hilfe zu holen (de Jong Gierveld, van Tilburg, \& Dykstra 2006). Eine solche Aufklärungskampagne muss so gestaltet sein, dass sich Frauen und Männer gleichermaßen angesprochen fühlen. Und es muss mit Augenmaß vorgegangen werden, um kein Stereotyp vom einsamen Alter zu bedienen und eine Verschlechterung der Vorstellungen vom Älterwerden zu vermeiden. Im nächsten Schritt sollten Maßnahmen ergriffen werden, die es mehr Menschen als bisher ermöglichen, sich aus ihrer Isolation oder Einsamkeit zu befreien. Dazu gehören beispielsweise niedrigschwellige Interaktions- und Teilhabeangebote im örtlichen Nahraum wie die Förderung von Nachbarschaftskontakten. Basierend auf unseren Analysen könnten sich diese Interventionen besonders auf das jüngere mittlere Lebensalter und das hohe Alter konzentrieren.

\section{Literatur}

Allan, G. (2008). Flexibility, friendship, and family. Personal Relationships, 15(1), 1-16.

Antonucci, T. C., Lansford, J. E., Akiyama, H., Smith, J., Baltes, M. M., Takahashi, K., Fuhrer, R., \& Dartigues, J. F. (2002). Differences between men and women in social relations, resource deficits, and depressive symptomatology during later life in four nations. Journal of Social Issues, 58(4), 767-783. doi: 10.1111/1540-4560.00289.

Baumeister, R. F., \& Leary, M. R. (1995). The need to belong - desire for interpersonal attachment as a fundamental human-motivation. Psychological Bulletin, 117(3), 497-529. doi: 10.1037/0033-2909.11 7.3.497. 
Böger, A., \& Huxhold, O. (2018). Age-Related Changes in Emotional Qualities of the Social Network From Middle Adulthood Into Old Age: How Do They Relate to the Experience of Loneliness? Psychology and Aging, 33(3), 482-496. doi: 10.1037/pag0000222.

Böger, A., Wetzel, M., \& Huxhold, O. (2017). Allein unter vielen oder zusammen ausgeschlossen: Einsamkeit und wahrgenommene soziale Exklusion in der zweiten Lebenshälfte. In: K. Mahne, J. K. Wolff, J. Simonson \& C. Tesch-Römer (Hrsg.) Altern im Wandel. Zwei Jahrzehnte Deutscher Alterssurvey (DEAS) (S. 273-285). Wiesbaden: Springer VS.

Carstensen, L. L., Isaacowitz, D. M., \& Charles, S. T. (1999). Taking time seriously - A theory of socioemotional selectivity. American Psychologist, 54(3), 165-181. doi: 10.1037//0003-066x.54.3.165.

Drinkuth, K. (2018). Die Einsamkeit breitet sich in Deutschland aus wie eine Epidemie. Tagesspiegel Online, 05. 03. 2018. Verfügbar unter https://www.tagesspiegel.de/weltspiegel/soziale-isolation-dieeinsamkeit-breitet-sich-in-deutschland-aus-wie-eine-epidemie/21035520.html [abgerufen am 11.12. 2018]

Engstler, H., \& Tesch-Römer, C. (2010). Lebensformen und Partnerschaft. In: A. Motel-Klingebiel, S. Wurm \& C. Tesch-Römer (Hrsg.) Altern im Wandel. Befunde des Deutschen Alterssurveys (DEAS) (S. 163-187). Stuttgart: Kohlhammer.

Fischer, C. S., \& Beresford, L. (2015). Changes in Support Networks in Late Middle Age: The Extension of Gender and Educational Differences. The Journals of Gerontology, Series B: Psychological Sciences and Social Sciences, 70(1), 123-131. doi: 10.1093/geronb/gbu057.

Freund, A. M., \& Ritter, J. O. (2009). Midlife Crisis: A Debate. Gerontology, 55(5), 582-591. doi: 10.11 $59 / 000227322$.

Hawkley, L. C., \& Cacioppo, J. T. (2010). Loneliness Matters: A Theoretical and Empirical Review of Consequences and Mechanisms. Annals of Behavioral Medicine, 4O(2), 218-227.

Hawthorne, G. (2006). Measuring Social Isolation in Older Adults: Development and Initial Validation of the Friendship Scale. Social Indicators Research, 77(3), 521-548. doi: 10.1007/s11205-005-7746-y.

Huxhold, O. (2019, eingereichtes Manuskript). Gauging Effects of Historical Changes on Aging Trajectories: The Increasing Importance of Friendships.

Huxhold, O., \& Fiori, K. L. (2018). Do demographic changes jeopardize social integration among aging adults living in rural regions? The Journals of Gerontology, Series B: Psychological Sciences and Social Sciences, gby008-gby008. doi: 10.1093/geronb/gby008.

Huxhold, O., Fiori, K. L., \& Windsor, T. D. (2013). The dynamic interplay of social network characteristics, subjective well-being and health: The costs and benefits of socio-emotional selectivity. Psychology and Aging, 28(1), 3-16. doi: 10.1037/a0030170.

Huxhold, O., Mahne, K., \& Naumann, D. (2010). Soziale Integration. In: A. Motel-Klingebiel, S. Wurm \& C. Tesch-Roemer (Hrsg.) Altern im Wandel. Befunde des Deutschen Alterssurveys (DEAS) (S. 215-232). Stuttgart: Kohlhammer.

Jong Gierveld J. de, Tilburg, T. G. van, \& Dykstra, P. A. (2006). Loneliness and social isolation. In: A. Vangelisti \& D. Perlman (Hrsg.) Handbook of personal relationships (S. 485-500). Cambridge, England: Cambridge University Press.

Levy, B. (2009). Stereotype embodiment: A psychosocial approach to aging. Current Directions in Psychological Science, 18(6), 332-336. doi: 10.1111/j.1467-8721.2009.01662.x.

Luo, Y., Hawkley, L. C., Waite, L. J., \& Cacioppo, J. T. (2012). Loneliness, health, and mortality in old age: A national longitudinal study. Social Science \& Medicine, 74(6), 907-914. doi: 10.1016/j.socscimed.20 11.11.028. 
Mahne, K., \& Huxhold, O. (2017). Nähe auf Distanz: Bleiben die Beziehungen zwischen älteren Eltern und ihren erwachsenen Kindern trotz wachsender Wohnentfernungen gut? In: K. Mahne, J. K. Wolff, J. Simonson \& C. Tesch-Römer (Hrsg.) Altern im Wandel. Zwei Jahrzehnte Deutscher Alterssurvey (DEAS) (S. 215-230). Wiesbaden: Springer VS.

Menkin, J. A., Robles, T. F., Gruenewald, T. L., Tanner, E. K., \& Seeman, T. E. (2017). Positive Expectations Regarding Aging Linked to More New Friends in Later Life. The Journals of Gerontology, Series B: Psychological Sciences and Social Sciences, 72(5), 771-781. doi: 10.1093/geronb/gbv118.

Miche, M., Huxhold, O., \& Stevens, N. L. (2013). A latent class analysis of friendship network types and their predictors in the second half of life. The Journals of Gerontology, Series B: Psychological Sciences and Social Sciences, 68(4), 644-652. doi: 10.1093/geronb/gbt041.

Perlman, D., \& Peplau, L. A. (1981). Toward a social psychology of loneliness. In: R. Gilmour \& S. Duck (Hrsg.) Personal Relationships: 3. Relationships in Disorder (S. 31-56). London: Academic Press.

Perrig-Chiello, P., Spahni, S., Hopflinger, F., \& Carr, D. (2016). Cohort and Gender Differences in Psychosocial Adjustment to Later-Life Widowhood. The Journals of Gerontology, Series B: Psychological Sciences and Social Sciences, 71(4), 765-774. doi: 10.1093/geronb/gbv004.

Sander, J., Schupp, J., \& Richter, D. (2017). Getting Together: Social Contact Frequency Across the Life Span. Developmental Psychology, 53(8), 1571-1588. doi: 10.1037/dev0000349.

Soest T. von, Luhmann, M., Hansen, T., \& Gerstorf, D. (2018). Development of loneliness in midlife and old age: Its nature and correlates. Journal of Personality and Social Psychology, Online First Publication. doi: $10.1037 /$ pspp0000219.

Stevens, N. L., \& Tilburg, T. G. van (2011). Cohort differences in having and retaining friends in personal networks in later life. Journal of Social and Personal Relationships, 28(1), 24-43. doi: 10.1177/026540 7510386191.

Suanet, B., \& Antonucci, T. C. (2017). Cohort Differences in Received Social Support in Later Life: The Role of Network Type. The Journals of Gerontology, Series B: Psychological Sciences and Social Sciences, 72(4), 706-715. doi: 10.1093/geronb/gbw075.

Suanet, B., \& Huxhold, O. (2018). Cohort Difference in Age-Related Trajectories in Network Size in Old Age: Are Networks Expanding ? The Journals of Gerontology, Series B: Psychological Sciences and Social Sciences, gbx166-gbx166. doi: 10.1093/geronb/gbx166.

Will, G. F. (2017). We have an epidemic of loneliness. How can we fix it? The Washington Post, 12. 10. 2017. Wrzus, C., Hanel, M., Wagner, J., \& Neyer, F. J. (2013). Social Network Changes and Life Events Across the Life Span: A Meta-Analysis. Psychological Bulletin, 139(1), 53-80. doi: 10.1037/a0028601. 
Open Access Dieses Kapitel wird unter der Creative Commons Namensnennung 4.0 International Lizenz (http:// creativecommons.org/licenses/by/4.0/deed.de) veröffentlicht, welche die Nutzung, Vervielfältigung, Bearbeitung, Verbreitung und Wiedergabe in jeglichem Medium und Format erlaubt, sofern Sie den/die ursprünglichen Autor(en) und die Quelle ordnungsgemäß nennen, einen Link zur Creative Commons Lizenz beifügen und angeben, ob Änderungen vorgenommen wurden.

Die in diesem Kapitel enthaltenen Bilder und sonstiges Drittmaterial unterliegen ebenfalls der genannten Creative Commons Lizenz, sofern sich aus der Abbildungslegende nichts anderes ergibt. Sofern das betreffende Material nicht unter der genannten Creative Commons Lizenz steht und die betreffende Handlung nicht nach gesetzlichen Vorschriften erlaubt ist, ist für die oben aufgeführten Weiterverwendungen des Materials die Einwilligung des jeweiligen Rechteinhabers einzuholen. 


\title{
6. Unbezahlte Sorgetätigkeiten von Frauen und Männern im Verlauf der zweiten Lebenshälfte
}

\author{
Daniela Klaus und Claudia Vogel
}

\section{Kernaussagen}

Die Wahrscheinlichkeit unbezahlter Sorgetätigkeiten steigt im Verlauf der zweiten Lebenshälfte zunächst an, sinkt danach bis zum 90. Lebensjahr wieder ab: Dabei zeigt sich für die Enkelbetreuung eine Konzentration auf die späte Erwerbsphase sowie auf das gesetzliche Renteneintrittsalter. Pflege- und Unterstützungsleistungen kommen ab dem 50. Lebensjahr bis zum Ende der Erwerbsphase besonders häufig vor, verteilen sich aber insgesamt gleichmäßiger über die zweite Lebenshälfte als die Enkelbetreuung.

Frauen übernehmen unbezahlte Sorgetätigkeiten mit höherer Wahrscheinlichkeit als Männer: Im Alter von 60 Jahren ist die Wahrscheinlichkeit der Enkelbetreuung für Frauen um zehn Prozent höher als für Männer. Bei Pflege- und Unterstützungsleistungen liegt der Unterschied zwischen Frauen und Männern bei sieben Prozent.

Frauen übernehmen unbezahlte Sorgetätigkeiten biografisch früher als Männer: Bei Frauen ist die Wahrscheinlichkeit für die Betreuung von Enkelkindern im Alter von 63 Jahren am höchsten, für Männer im Alter von 67 Jahren. Auch Pflege- und Unterstützungsleistungen werden von den Frauen früher erbracht als von den Männern, nämlich verstärkt im Alter von 57 Jahren gegenüber 61 Jahren bei den Männern.

Unbezahlte Sorgetätigkeiten von Frauen und Männern entwickeln sich im Kohortenvergleich unterschiedlich: Frauen und Männer späterer Geburtskohorten betreuen Enkelkinder in zunehmend höherem Alter. Pflege- und Unterstützungsleistungen werden bereits eher in der zweiten Lebenshälfte übernommen als bei früher geborenen Kohorten und zudem zunehmend häufiger. Geschlechterunterschiede bei der Enkelbetreuung nehmen über die Geburtskohorten hinweg ab, während Frauen weiterhin mit höherer Wahrscheinlichkeit und biografisch früher Pflege- und Unterstützungsleistungen übernehmen als Männer. 


\subsection{Einleitung}

Unbezahlte Arbeiten wie Hausarbeit und Sorgetätigkeiten für (Familien-)Angehörige, Nachbarinnen und Nachbarn sowie für Personen aus dem Freundes- oder Bekanntenkreis stoßen auf große Nachfrage. Sie werden von Privatpersonen in Privathaushalten erbracht und sie sind von hohem Wert: für das individuelle Wohlbefinden der Empfängerinnen und Empfänger, für die soziale Einbindung der beteiligten Personen, die Pflege ihrer Solidargemeinschaft sowie für die gesamtgesellschaftliche Wohlfahrtsproduktion. Eine Schätzung zur Wertschöpfung durch unbezahlte Arbeit weist aus, dass sie fast 40 Prozent der im Bruttoinlandsprodukt enthaltenen Bruttowertschöpfung ausmacht (2012/2013), wobei ihr Wert auf jährlich 987 Milliarden Euro geschätzt wird (Schwarz \& Schwahn 2016: 46). Der Großteil davon geht auf Arbeiten im Haus(-halt) und Garten zurück (79 Prozent) und weitere neun Prozent resultieren aus Pflege- und Betreuungsaufgaben (ebd.: 47).

Dabei wird unbezahlte Arbeit keineswegs nur in früheren Lebens- und Familienphasen erbracht. Auch Menschen mittleren und hohen Alters übernehmen solche Aufgaben in beträchtlichem Umfang. Neben (gegenseitigen) Hilfen bei Reparaturen, Garten- und Hausarbeit beinhaltet das auch die Unterstützung bedürftiger Personen. Das kann in Form finanzieller Zuwendungen für Familienmitglieder sein, als Betreuung und Versorgung von Enkelkindern oder als Fürsorge für Angehörige, die aufgrund gesundheitlicher Beschwerden in ihrer Alltagsorganisation eingeschränkt oder pflegebedürftig sind. So haben Auswertungen des Deutschen Alterssurveys (DEAS) gezeigt, dass die Übernahme von Enkelbetreuung nach einem rückläufigen Trend der letzten Jahre (von 34 Prozent im Jahr 1996 auf 25 Prozent im Jahr 2008) wieder zugewonnen hat: Im Jahr 2014 berichten etwa 30 Prozent aller Großeltern, dass sie Enkelkinder betreuen (Mahne \& Klaus 2017: 241). Auch ist der Anteil derer, die gesundheitlich beeinträchtigte Personen unterstützen oder pflegen, im Laufe der letzten Jahre gestiegen: von zwölf Prozent
(1996) auf 16 Prozent (2014) (Klaus \& Tesch-Römer 2017: 191).

Diese (erneut) wachsenden Betreuungs- und Unterstützungsquoten im privaten Bereich sind Antwort auf eine gestiegene Nachfrage. Diese hat bislang keine ausreichende Entlastung durch die Schaffung (ergänzender) professioneller und institutioneller Betreuungs- und Versorgungsmöglichkeiten erfahren. Hier findet die in ihren Grundzügen konservative Wohlfahrtspolitik Deutschlands ihren Ausdruck, die in den vergangenen Jahrzehnten nur punktuell modifiziert wurde. Insbesondere Sozial- und Pflegepolitik fußen weiterhin auf dem Subsidiaritätsprinzip, wonach bei Hilfebedürftigkeit die Erstverantwortung in der Familie liegt. So werden 1,4 der aktuell 2,9 Millionen Menschen, die im Sinne der Pflegeversicherung leistungsberechtigt sind, ausschließlich durch Angehörige im häuslichen Bereich versorgt (Statistisches Bundesamt 2017: 5). Darüber hinaus wird geschätzt, dass weitere 5,4 Millionen Menschen zwar nicht pflegebedürftig gemäß dieser Definition sind, dennoch auf Grund gesundheitlicher Einschränkungen auf regelmäßige Unterstützung angewiesen sind (Geyer \& Schulz 2014). Mehr noch für sie als für die im engeren Sinne Pflegebedürftigen ist davon auszugehen, dass deren Unterstützung überwiegend im privat-informellen $\mathrm{Be}$ reich organisiert und erbracht wird.

Zumindest einige Entlastungen brachten neben der Ausweitung der Pflegeversicherung vor allem familien- und arbeitsmarktpolitische Maßnahmen, welche die Förderung der Erwerbstätigkeit von Müttern zum Ziel haben, bei gleichzeitiger Verbesserung der Vereinbarkeit mit Sorgetätigkeiten (vgl. auch den Zweiten Bericht zur Gleichstellung von Frauen und Männern: Deutscher Bundestag 2017). So wurde der Ausbau von Kinderbetreuungseinrichtungen weiter vorangetrieben, was eine Verdoppelung der Betreuungsquote von Kindern unter drei Jahren von 16 auf 33 Prozent zwischen 2007 und 2017 brachte (Statistisches Bundesamt 2018: 117). Doch die Betreuungsdichte schwankt zwi- 
schen einzelnen Regionen stark. Vielerorts liegt die Anzahl verfügbarer Plätze unter der Nachfrage (Bundesministerium für Familie, Senioren, Frauen und Jugend 2018). Das hat zur Folge, dass der berufliche Wiedereinstieg der jungen Eltern - zumeist Mütter - aufgeschoben werden muss. Und so werden (wieder) verstärkt Betreuungsarrangements in der Familie und im privaten Umfeld gesucht. Das bezieht in erster Linie Großeltern ein, vor allem, wenn diese in der Nähe leben. Doch schränkt die in der späten Erwerbsphase angestiegene Frauenerwerbstätigkeit (Vogel \& Scherger 2018) gerade die Zeit- und Energieressourcen derer ein, die bislang typischerweise diese Sorgeaufgaben übernehmen, nämlich der Frauen.

\section{Geschlechterungleichheit bei der Ausübung unbezahlter Sorgetätigkeiten}

Frauen sind in deutlich größerem Umfang am Erbringen unbezahlter Sorgetätigkeiten beteiligt als Männer. Der für 2012/2013 über alle Altersgruppen hinweg ermittelte sogenannte Gender Care Gap liegt bei 52 Prozent (Deutscher Bundestag 2017: 95). Umgerechnet auf den täglichen Zeitaufwand bedeutet das, dass Frauen 87 Minuten mehr unbezahlte Sorgetätigkeiten leisten als Männer (ebd.: 96). Wie der Deutsche Alterssurvey eindrücklich belegt, sind es auch im mittleren und höheren Alter gerade die Frauen, die Leistungen dieser Art nicht nur häufiger, sondern auch zeitintensiver als Männer erbringen: Beispielsweise werden nach wie vor in einem Großteil der Partnerschaften ab dem 40. Lebensjahr anfallende Hausarbeiten überwiegend von den Frauen (61 Prozent) übernommen (Engstler \& Klaus 2017: 201). Auch die Enkelbetreuungs- und Unterstützungsquoten fallen unter 40- bis 85-jährigen Frauen statistisch signifikant höher aus (32 bzw. 19 Prozent) als unter den gleichaltrigen Männern (28 bzw. 13 Prozent) (Klaus \& Tesch-Römer 2017: 192; Mahne \& Klaus 2017: 242). Also auch für die zweite Lebenshälfte gilt: Haus-, Familien und Pflegearbeiten werden mehrheitlich von Frauen verantwortet.

\section{Unbezahlte Sorgetätigkeiten im Alternsverlauf}

Während Geschlechterunterschiede in der Übernahme von Sorgetätigkeiten gut belegt sind, liegen bislang kaum fundierte Informationen darüber vor, wie sich Sorgetätigkeiten über das mittlere bis ins hohe Erwachsenenalter hinweg verändern. Gibt es beispielsweise besonders beanspruchte Lebensphasen? Entsprechend wenig weiß man darüber, ob sich Frauen und Männer mit zunehmendem Alter und insbesondere nach Renteneintritt einander annähern oder nicht. Einzelne Studien und Statistiken legen nahe, dass ein Großteil der Aufgaben wie Enkelbetreuung oder Unterstützung älterer und unterstützungsbedürftiger Eltern in die späte Erwerbsphase fallen. Zwar ist das durchschnittliche Übergangsalter in die Großelternschaft in den letzten Jahren angestiegen, liegt aber mit 53 Jahren weiterhin im mittleren Erwachsenenalter (Mahne \& Klaus 2017: 236 und ähnliche und differenziertere Analysen bei Leopold \& Skopek 2015). Gut die Hälfte (53 Prozent) der privat Pflegeleistenden ist zwischen 40 und 64 Jahren alt (TNS Infratest Sozialforschung 2011: 27). Doch es wächst auch der Anteil hochaltriger Pflegepersonen (z. B. Runde, Giese, Kaphengst, \& Hess 2009; Rothgang, Iwansky, Müller, Sauer, \& Unger 2010). Überdies sind Empfängerinnen und Empfänger häufig bereits vor dem eintretenden Bedarf zeitintensiver Pflege auf Hilfe bei ihrer Alltagsbewältigung und Haushaltsorganisation angewiesen. Eine möglicherweise viele Jahre andauernde Unterstützungsbeziehung beginnt also nicht selten bereits in der Phase aktiver Erwerbstätigkeit der Unterstützungspersonen.

\section{Unbezahlte Sorgetätigkeiten und Erwerbstätigkeit}

Wie in frühen Lebens- und Familienphasen wird auch im mittleren und höheren Erwachsenenalter die stärkere Einbindung von Frauen in Hausund Sorgetätigkeiten von einer umfangreicheren 
Erwerbsbeteiligung der Männer begleitet. Doch selbst wenn die Gesamtarbeitszeit unbezahlter und bezahlter Arbeit von Frauen und Männern gleich ist (Hobler, Klenner, Pfahl, Sopp, \& Wagner 2017), impliziert diese geschlechtsspezifische Arbeitsteilung vielfältige Benachteiligungen für die Frauen. Offenkundig unterscheiden sich Erwerbsarbeit und unbezahlte Sorgetätigkeiten in ihrer monetären und damit auch gesellschaftlichen Gratifikation. Daraus resultiert nicht nur eine Ungleichverteilung sozialer Wertschätzung, sondern auch der finanziellen (Un-)Abhängigkeit und damit verbunden der Absicherung im Rentenalter. Die Übernahme von Kindererziehung und Pflegetätigkeiten in der Familie werden als Hauptrisikofaktoren für Armut im Alter genannt (Brettschneider \& Klammer 2016; Vogel \& Künemund 2018). Hinzu kommt - insbesondere für privat Pflegeleistende eine hohe gesundheitliche und soziale Belastung (Schwinger, Tsiasioti, \& Klauber 2016; Nowossadeck, Engstler, \& Klaus 2016; Engstler \& TeschRömer 2017; Bestmann, Wüstholz, \& Verheyen 2014). Sind Personen, die Fürsorgetätigkeiten leisten, zudem erwerbstätig, müssen beide Tätigkeitsbereiche miteinander vereinbart werden. Das geht oft mit zusätzlicher körperlicher sowie psychischer Beanspruchung der Sorgeleistenden einher. Nicht zu vergessen sind eingeschränkte Berufs- und Karrierechancen, wenn die Erwerbstätigkeit reduziert oder gar aufgegeben werden muss.

Das Spannungsverhältnis zwischen Erwerbsund Sorgetätigkeiten ist offenkundig. Es wurde vielfach untersucht und gesamtgesellschaftlich sowie politisch adressiert. Vor allem die Forschung zur Vereinbarkeit von Erwerbs- und Pflegetätigkeit hat gezeigt, dass sich die Übernahme von Pflege sowohl auf die Wahrscheinlichkeit, erwerbstätig zu bleiben als auch auf den Erwerbsumfang negativ auswirkt (u. a. Henz 2004; Bolin, Lindgren, \& Lundborg 2008; Keck 2012; Geyer 2016; Spiess \& Schneider 2003; Schäufele, Köhler, \& Hendlmeier 2016). So setzt nur die Hälfte (51 Prozent) der bei Pflegebeginn Erwerbstätigen ihre Erwerbstätigkeit in unverändertem Umfang fort, während 34 Prozent ihre Erwerbstätigkeit reduzieren und
15 Prozent sie ganz aufgegeben (TNS Infratest Sozialforschung 2011: 32). Dieser Effekt ist stärker bei Frauen als bei Männern (Frerk \& Leitner 2017: 276) und er ist nachhaltig: Ein beruflicher Wiedereinstieg nach der Pflegezeit ist unwahrscheinlich (Spiess \& Schneider 2003) und die Chancen hierfür sind gering (Keck 2016). Vergleichbare Untersuchungen zu Enkelbetreuung und informeller Unterstützung vor Pflegeeintritt gibt es kaum. Das mag daran liegen, dass hier keine Vereinbarkeitsproblematik vermutet wird. In der (gerontologischen) Familienforschung wird allenfalls - umgekehrt - der Einfluss von Erwerbstätigkeit auf die Übernahme dieser Sorgetätigkeiten untersucht. Die Befunde sind jedoch nicht eindeutig: Einigen Studien zufolge unterstützen und pflegen erwerbstätige erwachsene Kinder ihre Eltern seltener und weniger umfangreich als nicht erwerbstätige Kinder (Kalmijn \& Saraceno 2008; Leopold \& Raab 2011; Leopold, Raab, \& Engelhardt 2014). Erwerbstätige Großeltern betreuen ihre Enkel zwar nicht seltener, doch weniger umfangreich im Vergleich zu nicht erwerbstätigen Großeltern (Hank \& Buber 2009; Igel \& Szydlik 2011). In anderen Arbeiten erweist sich Erwerbstätigkeit in diesem Zusammenhang als unbedeutend (Deindl \& Brandt 2011; Klaus 2009: 235; Brandt, Haberkern, \& Szydlik 2009; Stuifbergen, van Delden, \& Dykstra 2008).

\section{Wandel in der Übernahme unbezahlter Sorgetätigkeiten}

Unbezahlte Sorgetätigkeiten haben im mittleren und höheren Lebensalter an Bedeutung gewonnen. Dabei hat sich die Nachfrage nach Betreuungsund Pflegeleistungen ebenso erhöht wie das Interesse, ältere Arbeitnehmerinnen und Arbeiternehmer so lang wie möglich im Beruf zu halten. Diese gesellschaftliche Entwicklung spiegelt sich auch in den individuellen Wünschen der Menschen wider: erwerbstätig zu sein und sich (zugleich) um unterstützungsbedürftige (Familien-)Angehörige kümmern zu können. Es ist anzunehmen, dass die gestiegene Bereitstellung und Organisation von 
Betreuung und Unterstützung im privaten Bereich durch die gestiegene Ressourcenausstattung der älteren Menschen begünstigt wird, die heute besser ausgebildet und gesünder sind als etwa noch vor 20 Jahren. Beispielsweise zeigen Untersuchungen, dass Menschen mit höherer Bildung anteilig häufiger Unterstützungsleistungen für Verwandte und nicht-verwandte Personen erbringen als Menschen mit geringer Bildung (Klaus \& Tesch-Römer 2017: 192; Vogel \& Tesch-Römer 2017: 263) und eine gute Gesundheit ist nachweislich Vorbedingung für das Leisten instrumenteller Hilfen wie Betreuung und Pflege. Allerdings sind Probleme der Vereinbarkeit von Erwerbs- und Sorgetätigkeiten ebenso bekannt wie die Tatsache, dass es häufiger Frauen sind, die vor dieser Aufgabe stehen. Weniger weiß man jedoch über die Dynamik der Geschlechterunterschiede bezüglich der Übernahme unbezahlter Sorgetätigkeiten im Verlauf des Älterwerdens sowie über einen möglichen gesellschaftlichen Wandel geschlechtsspezifischer Alternsverläufe über nachfolgende Geburtskohorten.

\subsection{Methoden}

\section{Stichprobe}

Zur Beantwortung der aufgeworfenen Fragestellungen werden die Daten des Deutschen Alterssurveys (DEAS) der Jahre 1996 bis 2017 verwendet. Im Verlauf der bislang sechs Erhebungswellen wurden 20129 Personen befragt, für die 39446 Beobachtungen vorliegen (zur Stichprobenziehung und Datenbasis siehe Kapitel 2 Design, Inhalte und Methodik des Deutschen Alterssurveys in diesem Band). Die Teilnahmebereitschaft in der Erstbefragung sowie die Wiederbefragungsquoten bewegen sich im Durchschnitt europäischer Studien. Doch da sowohl in der Erst- als auch in den Wiederholungsbefragungen Personen mit bestimmten Merkmalskombinationen jeweils unterschiedlich große Wahrscheinlichkeiten der (Wieder-)Teil-

\section{Fragestellungen}

Im vorliegenden Beitrag wird die Übernahme von unterschiedlichen Sorgetätigkeiten im Alternsverlauf dargestellt - getrennt für Frauen und Männer sowie im Kohortenvergleich. Im Fokus stehen zwei Sorgetätigkeiten, die typisch für die mittlere und hohe Lebensphase sind: die Betreuung von Enkelkindern sowie die Bereitstellung von Betreuung, Unterstützung oder Pflege für gesundheitlich eingeschränkte Personen. Die zwei Leitfragen lauten:

1) Bestehen aufgrund einer anhaltenden geschlechtsspezifischen Arbeitsteilung Unterschiede zwischen Frauen und Männern in der Übernahme von Sorgetätigkeiten im Verlauf der zweiten Lebenshälfte?

2) Deutet sich infolge des demografischen Wandels sowie der Veränderungen im Erwerbsverhalten der vergangenen Jahrzehnte eine Veränderung der geschlechtsspezifischen Muster und Verläufe in der Übernahme unbezahlter Sorgetätigkeiten an?

nahme haben, ist die verwendete Panelstichprobe nicht frei von Selektivität. Zwei Vorkehrungen wurden getroffen, um einen möglichen verzerrenden Einfluss der Selektivität auf Analysen und Schätzungen zu reduzieren: (1) Zum Ausgleich der geschichteten Stichprobenziehung in der Erstbefragung wird in allen Modellen neben dem Lebensalter, das die Zeitvariable darstellt, für $\mathrm{Ge}$ schlecht und Region (Ost- vs. Westdeutschland) kontrolliert. (2) Um die selektive Teilnahme in den Wiederbefragungen abzumildern, gehen auch die Untersuchungspersonen in die Schätzungen ein, die nur einmal an der Studie teilgenommen haben. 


\section{Indikatoren}

Über alle Befragungswellen hinweg wurden (nahezu) identische Fragen zur Übernahme von Enkelbetreuung sowie zur Übernahme von Pflege und Unterstützung für Andere gestellt. Die Kategorie „Andere“ umfasst sowohl Verwandte als auch nicht-verwandte Personen aus dem Freundes- und Bekanntenkreis sowie aus der Nachbarschaft. Entsprechend können vergleichbare Indikatoren erstellt werden. Für beide Sorgetätigkeiten werden dichotome Indikatoren gebildet. Diese geben an, ob zum Zeitpunkt der Befragung (1) Enkelkinder betreut werden (Wert 1) oder nicht (Wert 0 ) und ob (2) andere Personen auf Grund deren schlechten Gesundheitszustandes betreut, gepflegt oder anderweitig regelmäßig unterstützt werden (Wert 1) oder nicht (Wert 0). Dabei zeigt der Wert 0 entweder an, dass ein Enkelkind oder ein unterstützungsbedürftiger Angehöriger zwar vorhanden ist, aber nicht betreut wird. Oder der Wert 0 verweist darauf, dass es entsprechende potenzielle Empfängerinnen oder Empfänger nicht gibt, etwa weil die Befragungsperson keine Enkel hat. Somit beinhalten die Indikatoren eine sich möglicherweise geänderte Nachfrage nach diesen Sorgetätigkeiten, etwa weil in bestimmten Lebensphasen zunehmend mehr Personen (noch) keine Enkel haben. Im Falle der Pflege- und Unterstützungstätigkeit wird zusätzlich erfragt, wie viel Zeit für alle in diesem Sinne unterstützten Personen aufgewendet wird. Der hierzu generierte Indikator gibt den Wert in Wochenstunden an.

Um die Alternsverläufe der Ausübung dieser Tätigkeiten untersuchen und abbilden zu können, wird das Alter der Befragungsperson zum jeweiligen Befragungszeitpunkt berücksichtigt. Diese Variable geht als die zentrale Zeitvariable in die Analysen ein. Dabei kann sich die Wahrscheinlichkeit von Sorgetätigkeiten linear mit zunehmendem Alter verändern, aber auch nicht-linear verlaufen: etwa in Form einer Kurve, wenn ein Anstieg im frühen mittleren Erwachsenenalter im hohen Alter in einen Rückgang übergeht. Um das zu prüfen, geht das Alter sowohl in linearer als auch in quadrierter Form in die Modellschätzungen ein.

Um festzustellen, ob sich die Alternseffekte für Frauen und Männer unterscheiden, wird das $\mathrm{Ge}$ schlecht der Befragungsperson berücksichtigt. $\mathrm{Ob}$ mögliche Geschlechterunterschiede einem gesellschaftlichen Wandel unterliegen oder nicht, wird über die Aufnahme der Kohortenzugehörigkeit getestet. Es werden drei zentrale Geburtskohorten berücksichtigt, für die über eine größtmögliche Altersspanne empirische Beobachtungen (Messungen) vorliegen: zwischen 1930 und 1939 Geborene, von 1940 bis 1949 Geborene und von 1950 bis 1959 Geborene.

\section{Statistisches Analyseverfahren}

Die Schätzung der Alternsverläufe erfolgt anhand von Random-Effects-Panelregressionen (z. B. Brüderl 2010; Schröder 2007). Dieses Analyseverfahren nutzt alle vorliegenden Datenpunkte zwischen 1996 und 2017, das heißt alle Beobachtungen aller Studienteilnehmerinnen und -teilnehmer. Anders als bei Regressionen, die allein auf Querschnittdaten basieren (d.h. einmalige Befragung von Personen) geht demnach auch die Entwicklung (Stabilität oder Veränderung) der mehrfach befragten Personen ab dem Alter 40 in die Schätzung ein. Deshalb sind Panelregressionsmodelle verlässlicher und effizienter als querschnittliche Schätzmodelle, wenn es um die Schätzung von Alternsverläufen in den interessierenden Lebensbereichen geht (hier über die abgebildeten Alternsverläufe illustriert).

Für die beiden dichotom (das heißt in Form von ja-/nein-Angaben) vorliegenden Indikatoren der Sorgetätigkeit werden logistische Panelregressionen berechnet. Diese schätzen die Wahrscheinlichkeit, mit der die eine oder andere Sorgetätigkeit über den Alternsverlauf hinweg ausgeübt wird. Dabei wird nicht nur geprüft, ob die Ausübung einer Sorgetätigkeit sich überhaupt mit dem Alter verändert, sondern auch, welche Form der Alternsverlauf annimmt. Die auf Basis der Panelregression 
geschätzten Wahrscheinlichkeiten der Sorgetätigkeit werden grafisch dargestellt.

Um festzustellen, ob sich Frauen und Männer unterscheiden, wird Geschlecht als Variable aufgenommen. In den Schätzungen werden jeweils zwei Prüfungen vorgenommen: (1) Erstens wird getestet, ob sich Frauen und Männer hinsichtlich ihres Niveaus der Ausübung der Sorgetätigkeiten unterscheiden. Dabei wird die Signifikanzprüfung durchweg für das 60. Lebensjahr vorgenommen. Dieses Alter liegt etwas unter dem mittleren Alter der Gesamtstichprobe und zudem in der inhaltlich bedeutsamen späten Erwerbsphase. (2) Zweitens wird geprüft, ob sich die Verläufe zwischen den Geschlechtern unterscheiden. Das kann sich beispielsweise an unterschiedlich starken Anstie-

\subsection{Ergebnisse}

\subsubsection{Enkelbetreuung im Verlauf der zweiten Lebenshälfte}

Frauen betreuen Enkelkinder früher im Verlauf der zweiten Lebenshälfte sowie im Alter von 60 Jahren mit höherer Wahrscheinlichkeit als Männer.

Die Abbildung 6-1 zeigt die Enkelbetreuung im Verlauf des Älterwerdens. Dabei wird ein umgekehrt u-förmiger Verlauf zwischen dem 40. und dem 90. Lebensjahr erkennbar: Die Enkelbetreuung kommt zu Beginn und zum Ende der betrachteten Altersspanne praktisch nicht vor. Die Betreuungswahrscheinlichkeit liegt hier bei jeweils null Prozent. Sie häuft sich im 7. Lebensjahrzehnt. Ihren Höchstwert erreicht sie im Alter von 65 Jahren: Wenn Personen dieses Lebensalter erreichen, dann liegt den Schätzungen zufolge die durchschnittliche Wahrscheinlichkeit, mindestens ein Enkelkind zu betreuen, bei 24 Prozent.

Doch das gilt nicht gleichermaßen für Frauen und Männer: Im Alter von 60 Jahren geht die Wahrscheinlichkeit der Enkelbetreuung um zehn Prozentpunkte auseinander (Frauen: 26 Prozent, gen zeigen oder auch darin, dass die durchschnittliche Wahrscheinlichkeit der Übernahme von Sorgetätigkeiten in jeweils unterschiedlichem Alter ihr Maximum erreicht. Hierzu wird geprüft, ob die Interaktion zwischen Alter und Geschlecht statistisch signifikant ist. Mögliche Geschlechterunterschiede werden zudem für die drei ausgewählten Geburtskohorten vergleichend betrachtet, um zu prüfen, ob sich diese Geschlechterunterschiede in den Alternsverläufen verändert haben. Das erfolgt über eine zusätzliche Interaktion von Geschlecht und Alter mit der Geburtskohortenzugehörigkeit. Zur Ermittlung der statistischen Signifikanz wird in allen Analysen das Signifikanzniveau auf fünf Prozent $(\mathrm{p}<0,05)$ gesetzt.

Männer: 16 Prozent). Ihren Höhepunkt erreicht diese Diskrepanz bereits im 56. Lebensjahr: Hier unterscheidet sich die Wahrscheinlichkeit von Enkelbetreuung zwischen den Geschlechtern um elf Prozentpunkte. Spätestens mit Beginn des Beobachtungszeitraumes setzt eine Spreizung der Verläufe ein: Während die Wahrscheinlichkeit bei den Frauen schon ab dem 40. Lebensjahr sichtbar ansteigt, setzt eine vergleichbar starke Zunahme bei den Männern erst ca. fünf Jahre später ein. Zudem betreuen Frauen früher im Verlauf ihrer zweiten Lebenshälfte als Männer. Ihren Maximalwert erreichen Frauen mit 63 Jahren (27 Prozent), Männer mit 67 Jahren (21 Prozent). Die nach dem 56. Lebensjahr einsetzende Angleichung der Alternsverläufe ist mit Anfang 70 erreicht: Danach unterscheiden sich die Geschlechter in der Wahrscheinlichkeit von Enkelkinderbetreuung statistisch nicht mehr signifikant.

Enkelkinderbetreuung wird von nachfolgenden Kohorten in zunehmend höherem Alter übernommen. 
Abbildung 6-1 Wahrscheinlichkeit der Enkelbetreuung bei Frauen und Männern im Alternsverlauf

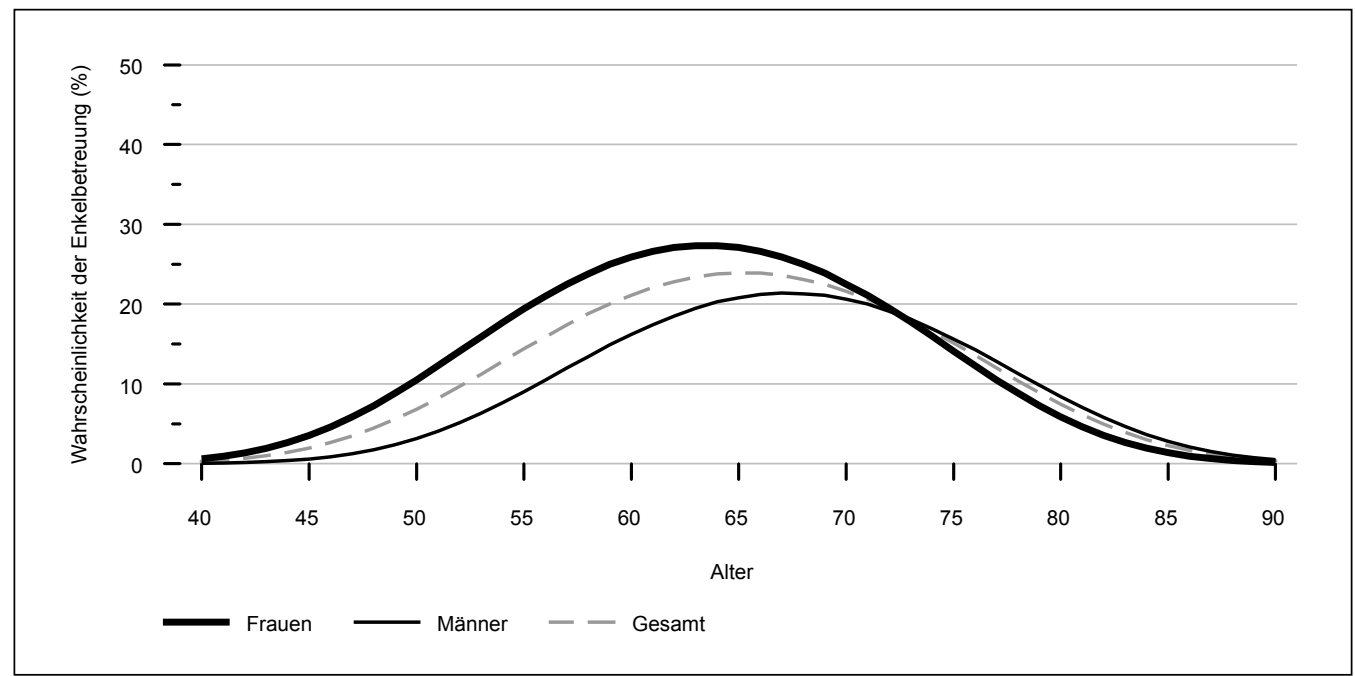

Quelle: DEAS 1996-2017, Schätzungen basieren auf n = 20114 Studienteilnehmenden mit insgesamt $\mathrm{n}=39361$ Beobachtungen, kontrolliert für Region.

Bei Frauen steigt die Wahrscheinlichkeit, Enkelkinder zu betreuen auf ein Maximum von 27 Prozent an (mit 63 Jahren), um danach wieder zu sinken. Bei Männern steigt die Wahrscheinlichkeit auf ein Maximum von 21 Prozent an (mit 67 Jahren). Im Alter von 90 Jahren liegt die Wahrscheinlichkeit der Enkelkinderbetreuung sowohl bei Frauen als auch bei Männern bei null. Der Unterschied zwischen Frauen und Männern in der Wahrscheinlichkeit im Alter von 6o Jahren ist signifikant. Ebenso unterscheidet sich der geschätzte Verlauf der Wahrscheinlichkeit der Enkelbetreuung zwischen Frauen und Männern signifikant.

Die Übernahme der Enkelbetreuung im Alternsverlauf hat sich über die betrachteten Geburtskohorten hinweg signifikant verändert; vor allem findet sie vermehrt in deutlich höherem Alter statt (Abbildung 6-2). Die Wahrscheinlichkeit, im Alter von 60 Jahren mindestens ein Enkelkind zu betreuen, sinkt von 25 Prozent in der Kohorte der zwischen 1930 und 1939 Geborenen, über 23 Prozent in der mittleren Geburtskohorte auf 20 Prozent in der Kohorte der zwischen 1950 und 1959 Geborenen. Die maximale Betreuungswahrscheinlichkeit wird in zunehmend höherem Alter erreicht: In der Kohorte der in den 1930er Jahren Geborenen im Lebensalter von 60 Jahren (25 Prozent), in der mittleren Geburtskohorte im Lebensalter von 66 Jahren (25 Prozent). In der Kohorte der zwischen 1950 und 1959 Geborenen liegt sie vermutlich sogar außerhalb des Beobachtungszeitraumes: Unter der Annahme, dass sich der Kohor- tenwandel so fortsetzt wie auf Grundlage der vorliegenden Daten geschätzt, erreicht diese Kohorte ihre höchste Betreuungswahrscheinlichkeit im Alter von 70 Jahren: mit 28 Prozent (diese voraussichtliche zukünftige Entwicklung ist nicht abgebildet).

Frauen und Männer betreuen Enkelkinder mit zunehmend gleicher Wahrscheinlichkeit.

Eine nach Geburtskohorten getrennte Betrachtung legt einen Rückgang der Geschlechterunterschiede bei aufeinander folgenden Kohorten nahe (Abbildung 6-3). Diese Angleichung über die Kohorten hinweg zeigt sich in der Wahrscheinlichkeit der Enkelbetreuung: In der Kohorte der in den 1930er Jahren Geborenen fällt der Geschlechterunterschied im Alter von 60 Jahren mit 15 Prozentpunkten am größten aus. In beiden Nachfolgekohorten 
Abbildung 6-2 Wahrscheinlichkeit der Enkelbetreuung nach Kohorten im Alternsverlauf

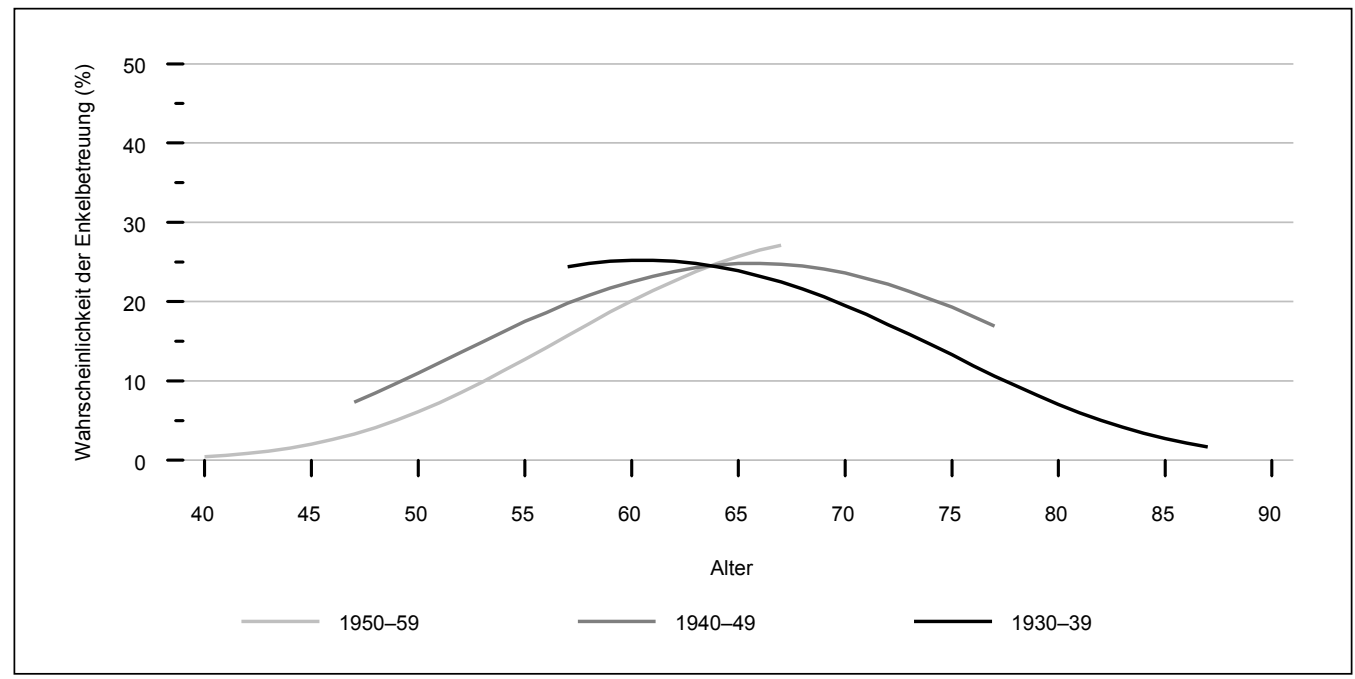

Quelle: DEAS 1996-2017, Schätzungen beruhen auf $n=13995$ Studienteilnehmenden mit insgesamt $n=29198$ Beobachtungen, kontrolliert für Region und Geschlecht.

Für die Kohorte der 1930 bis 1939 Geborenen steigt die Wahrscheinlichkeit der Enkelbetreuung bis zum Alter von 6o Jahren an (25 Prozent). Für die zwischen 1940 und 1949 geborenen Personen ist bis zum Alter von 66 Jahren (25 Prozent) ein Anstieg zu beobachten. Für die Kohorte der 1950 bis 1959 Geborenen ist ein Anstieg der Wahrscheinlichkeit der Enkelbetreuung bis zum Alter von 67 Jahren (27 Prozent) zu beobachten. Der Unterschied zwischen den Geburtskohorten in der durchschnittlichen Wahrscheinlichkeit der Übernahme von Enkelkinderbetreuung im Alter von 6o Jahren ist signifikant. Ebenso unterscheidet sich der geschätzte Verlauf der Wahrscheinlichkeit der Enkelbetreuung zwischen den Kohorten signifikant.

ist er jeweils kleiner: elf Prozentpunkte bei den in den 1940er Jahren Geborenen und acht Prozentpunkte bei den in den 1950er Jahren Geborenen. Hinter der Annäherung des Betreuungsverhaltens über die Kohorten hinweg stehen unterschiedliche Veränderungen bei Frauen und Männern. Zwar zeigt sich bei beiden Geschlechtern im Alter von 60 Jahren eine Abnahme der Betreuungswahrscheinlichkeit über nachfolgende Kohorten. Doch fällt dieser Rückgang bei den Frauen deutlich größer aus (von 33 Prozent in der Kohorte der 1930 bis 1939 Geborenen über 28 Prozent auf 24 Prozent in der Kohorte der 1950 bis 1959 Geborenen) als bei den Männern (von 18 Prozent in der Kohorte der 1930 bis 1939 Geborenen über 17 Prozent auf 16 Prozent in der Kohorte der 1950 bis 1959 Geborenen). Ein Vergleich der Maximalwerte belegt ebenfalls, dass Frauen - im Vergleich zu Männern - zunehmend mit geringerer Wahr- scheinlichkeit Enkelkinder betreuen: Bei ihnen sinkt der Maximalwert von 34 Prozent (Kohorte 1930-1939) auf 29 Prozent (Kohorte 1950-1959). Männer der später geborenen Geburtskohorten betreuen sogar mit etwas größerer Wahrscheinlichkeit als ihre Vorgänger: Ihr Maximalwert steigt in der Kohortenabfolge von 20 Prozent auf 28 Prozent.

Die Geschlechterdiskrepanz im Alternsverlauf bleibt hingegen unverändert. Hier kommt es zu keiner Annäherung zwischen Frauen und Männern. Zwar werden Enkel in einem zunehmend höheren Alter betreut, allerdings findet diese Entwicklung für beide Geschlechter gleichermaßen statt. Sowohl Frauen als auch Männern jüngerer Geburtskohorten betreuen später in der zweiten Lebenshälfte: Lag das Maximum in der Kohorte der in den 1930er geborenen Frauen bei 56 Jahren, so stieg es in der Kohorte der 1940 bis 1949 gebo- 
Abbildung 6-3 Wahrscheinlichkeit der Enkelbetreuung bei Frauen und Männern nach Kohorten im Alternsverlauf

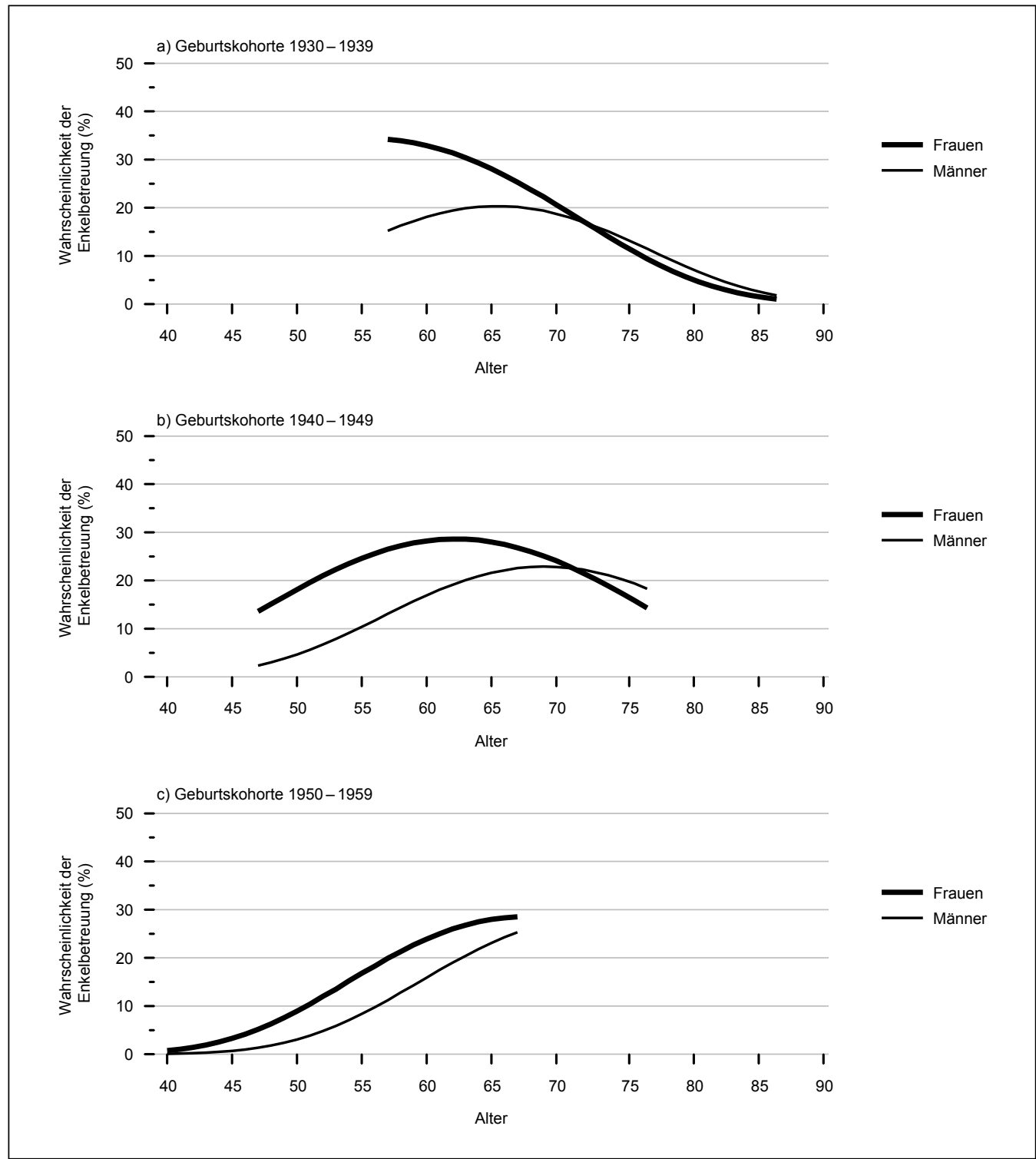

Quelle: DEAS 1996-2017, Schätzungen basieren auf n = 13995 Studienteilnehmenden mit insgesamt n = 29198 Beobachtungen, kontrolliert für Region.

In der Kohorte der 1930 bis 1939 Geborenen liegt die Wahrscheinlichkeit der Enkelbetreuung im Alter von 6o Jahren bei 33 Prozent bei den Frauen und bei 18 Prozent bei den Männern. Dieser Geschlechterunterschied reduziert sich über die nachfolgenden Kohorten hinweg signifikant: In der Kohorte der 1950 bis 1959 Geborenen beträgt die Wahrscheinlichkeit der Enkelbetreuung im Alter von 6o Jahren 24 Prozent bei den Frauen und 16 Prozent bei den Männern. Der geschätzte geschlechtsspezifische Verlauf der Wahrscheinlichkeit der Enkelbetreuung verändert sich über die Kohorten hinweg nicht signifikant: Geschlechterunterschiede im Alternsverlauf zeigen sich in allen drei betrachteten Kohorten gleichermaßen. 
renen Frauen auf 62 Jahre und in der Kohorte der 1950 bis 1959 geborenen Frauen lag es bereits bei 67 Jahren. In ähnlichem Umfang verläuft die Verlagerung bei den Männern: In der Kohorte 19301939 erreichen sie ihren Maximalwert mit 65 Jahren; in der Kohorte 1940-1949 mit 69 Jahren und in der jüngsten Kohorte 1950-1959 erst mit 73 Jahren. Damit bleibt der Geschlechterunterschied im Verlauf über die Kohorten hinweg erhalten. Das heißt, auch in der jüngsten Geburtskohorte betreuen Frauen deutlich früher als Männer.

\subsubsection{Unterstützung und Pflege im Verlauf der zweiten Lebenshälfte}

Frauen leisten Unterstützung und Pflege früher im Verlauf der zweiten Lebenshälfte als Männer sowie im Alter von 60 Jahren mit höherer Wahrscheinlichkeit.

Anders als die Enkelbetreuung, die sich auf eine bestimmte Lebensphase der älteren Personen konzentriert, verteilt sich die Unterstützung und Pflege von Angehörigen stärker über die gesamte zweite Lebenshälfte (Abbildung 6-4). Das gilt mehr noch für Männer als für Frauen. Doch auch in diesem Bereich der Sorgetätigkeiten kommt es im Verlauf des Älterwerdens zunächst zu einem Anstieg, der noch vor dem Erreichen des offiziellen Rentenalters in einen Rückgang übergeht. Die Betrachtung über beide Geschlechter hinweg zeigt, dass im Alter von 40 Jahren die Wahrscheinlichkeit, gesundheitlich eingeschränkte Angehörige zu unterstützen, zu betreuen und/oder zu pflegen bei zehn Prozent liegt. Im weiteren Alternsverlauf gewinnen diese Tätigkeiten an Bedeutung, um sich im Lebensalter von Mitte 50 bis Mitte $60 \mathrm{zu}$ häufen: Zwischen dem 54. und dem 65. Lebensjahr bewegt sich die Wahrscheinlichkeit auf einem Plateau von 16 Prozent. Danach setzt ein Rückgang ein: Dieser verläuft weniger steil als bei der Enkelbetreuung und auch mit 90 Jahren liegt die Wahrscheinlichkeit zu unterstützen und/oder zu pflegen noch bei fünf Prozent.
In Analogie zur Enkelbetreuung zeigen sich auch hier deutliche Geschlechterunterschiede sowohl im Niveau als auch im Verlauf des Älterwerdens: Männer leisten im Alter von 60 Jahren durchschnittlich seltener Unterstützung und Pflege für Verwandte und Personen aus dem Freundes- und Bekanntenkreis oder der Nachbarschaft als dies Frauen tun. Die Wahrscheinlichkeit liegt bei Frauen, wenn sie das 60. Lebensjahr erreichen, bei 20 Prozent; bei Männern bei 13 Prozent. Im höheren Alter kommt es zu einer allmählichen Angleichung: Ab dem 77. Lebensjahr zeigen sich in der Wahrscheinlichkeit des Unterstützungsverhaltens keine signifikanten Unterschiede mehr zwischen Frauen und Männern. Abgesehen von dieser Geschlechterdiskrepanz unterscheiden sich auch die Alternsverläufe. So nimmt die Wahrscheinlichkeit für Unterstützung und Pflege im Verlauf der späten Erwerbsphase bei Frauen deutlich zu: zwischen dem 40. und dem 57. Lebensjahr von zwölf Prozent auf 20 Prozent (Maximum). Danach nimmt die Wahrscheinlichkeit erheblich ab: auf drei Prozent im Alter von 90 Jahren. Anders sieht es für die Männer aus, für die Anstieg und Rückgang deutlich milder ausgeprägt sind: Im Alter von 40 liegt ihre Wahrscheinlichkeit bei acht Prozent, erreicht mit 61 Jahren ihren Höhepunkt (13 Prozent) und geht bis zum Alter von 90 Jahren auf sechs Prozent zurück. Die Kurve entlang des Älterwerdens fällt vergleichsweise flach aus. Dieser Sorgetätigkeit gehen Männer also nicht nur seltener nach, ihre Übernahmewahrscheinlichkeit verändert sich auch weniger mit dem Alter. Anders ist es bei den Frauen: Für sie stellt sich die späte Erwerbsphase als besonders beanspruchte Lebensphase heraus.

In später geborenen Kohorten wird Unterstützung und Pflege zunehmend früher im Verlauf der zweiten Lebenshälfte geleistet und ihre Wahrscheinlichkeit im Alter von 60 Jahren nimmt $z u$.

Unabhängig vom Geschlecht nimmt über die betrachteten Geburtskohorten hinweg die Bereitstellung von Unterstützung und Pflege für kranke 
Abbildung 6-4 Wahrscheinlichkeit gesundheitsbedingter Unterstützung und Pflege bei Frauen und Männern im Alternsverlauf

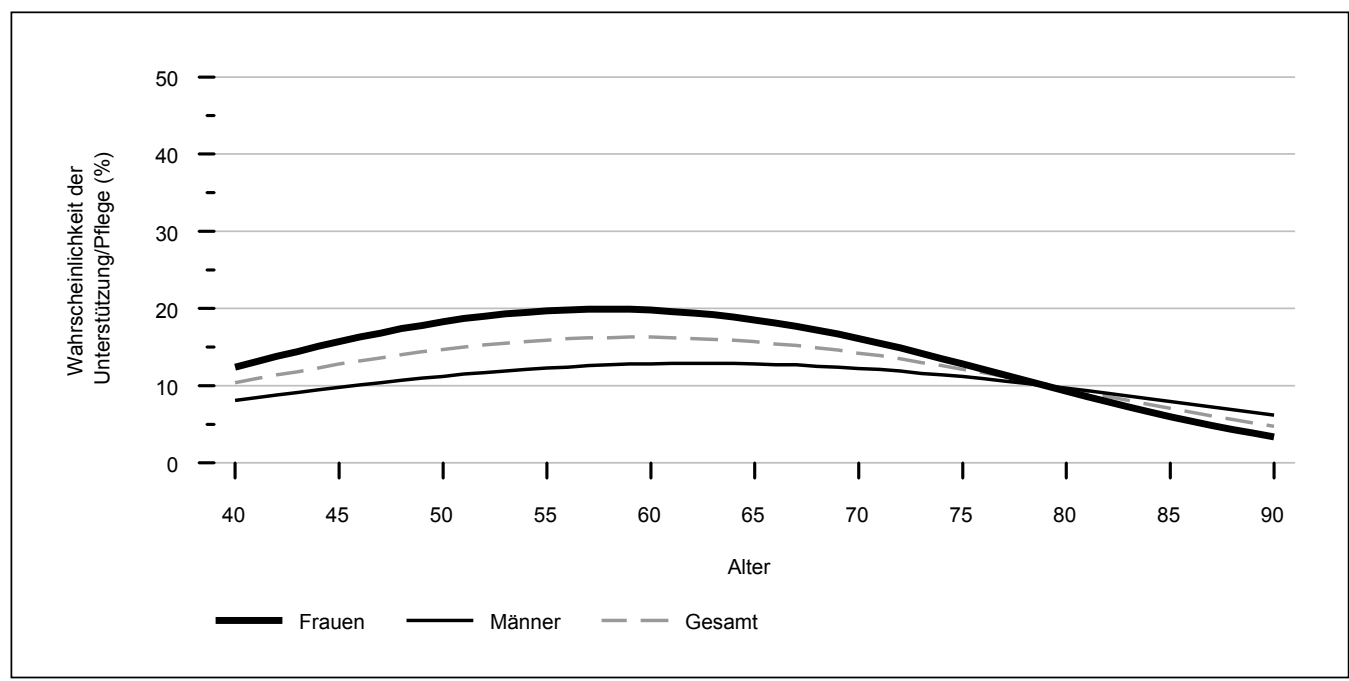

Quelle: DEAS 1996-2017, Schätzungen basieren auf $n=20114$ Studienteilnehmenden mit insgesamt n = 39342 Beobachtungen, kontrolliert für Region.

Bei Frauen steigt die Wahrscheinlichkeit, Angehörige zu unterstützen und/oder zu pflegen von zwölf Prozent (mit 40 Jahren), auf 20 Prozent (mit 57 Jahren) und geht danach zurück auf drei Prozent (mit 90 Jahren). Bei den Männern steigt die Wahrscheinlichkeit von acht Prozent (mit 40 Jahren) auf ${ }_{13}$ Prozent (mit 61 Jahren). Nach diesem Höchstwert geht sie auf sechs Prozent zurück (mit 90 Jahren). Sowohl der Unterschied in der Wahrscheinlichkeit im Alter von 6o Jahren als auch im geschätzten Alternsverlauf zwischen Frauen und Männern sind signifikant.

Angehörige zu (Abbildung 6-5). Diese Unterstützungsleistung wird im Alter von 60 Jahren mit einer Wahrscheinlichkeit von zwölf Prozent bei den in den 1930ern Geborenen übernommen; die Wahrscheinlichkeit beträgt 16 Prozent bei den in den 1940ern Geborenen und 19 Prozent bei den in den 1950er Jahren Geborenen.

Doch nicht nur die Wahrscheinlichkeit dieser Sorgetätigkeit ist gestiegen, auch der Alternsverlauf hat sich über die Kohorten hinweg stark verändert: Der umgekehrt u-förmige Verlauf, der in der Kohorte der in den 1950er Jahren Geborenen deutlich ausgeprägt ist, ist signifikant verschieden vom Verlauf der Kohorte der 1930 bis 1939 Geborenen. So erreicht die Unterstützungswahrscheinlichkeit bei den zwischen 1950 und 1959 Geborenen im Alter von 61 Jahren ihren höchsten Wert (19 Prozent). In diesem Alter bewegt sich die
Wahrscheinlichkeit der 1930er Geburtskohorte auf ihren Tiefstwert zu, den sie im Alter von 69 Jahren mit elf Prozent erreicht. Zugleich zeigt sich für diese Geburtskohorte der 1930 bis 1939 Geborenen eine vergleichsweise geringere Veränderung mit dem Alter: Unterstützung und/oder Pflege für gesundheitlich eingeschränkte Angehörige werden hier in jedem Alter der zweiten Lebenshälfte mit etwa gleich großer Wahrscheinlichkeit erbracht. In der Kohorte der 1950 bis 1959 Geborenen hingegen konzentrieren sich diese Sorgetätigkeiten vor allem auf das Alter um die 60, um danach deutlicher zurückzugehen als noch in der vorangehenden Kohorte der 1940 bis 1949 Geborenen.

Geschlechtsspezifische Unterschiede im Bereich Unterstützung und Pflege bleiben über nachfolgende Geburtskohorten unverändert. 
Abbildung 6-5 Wahrscheinlichkeit gesundheitsbedingter Unterstützung und Pflege nach Kohorten im Alternsverlauf

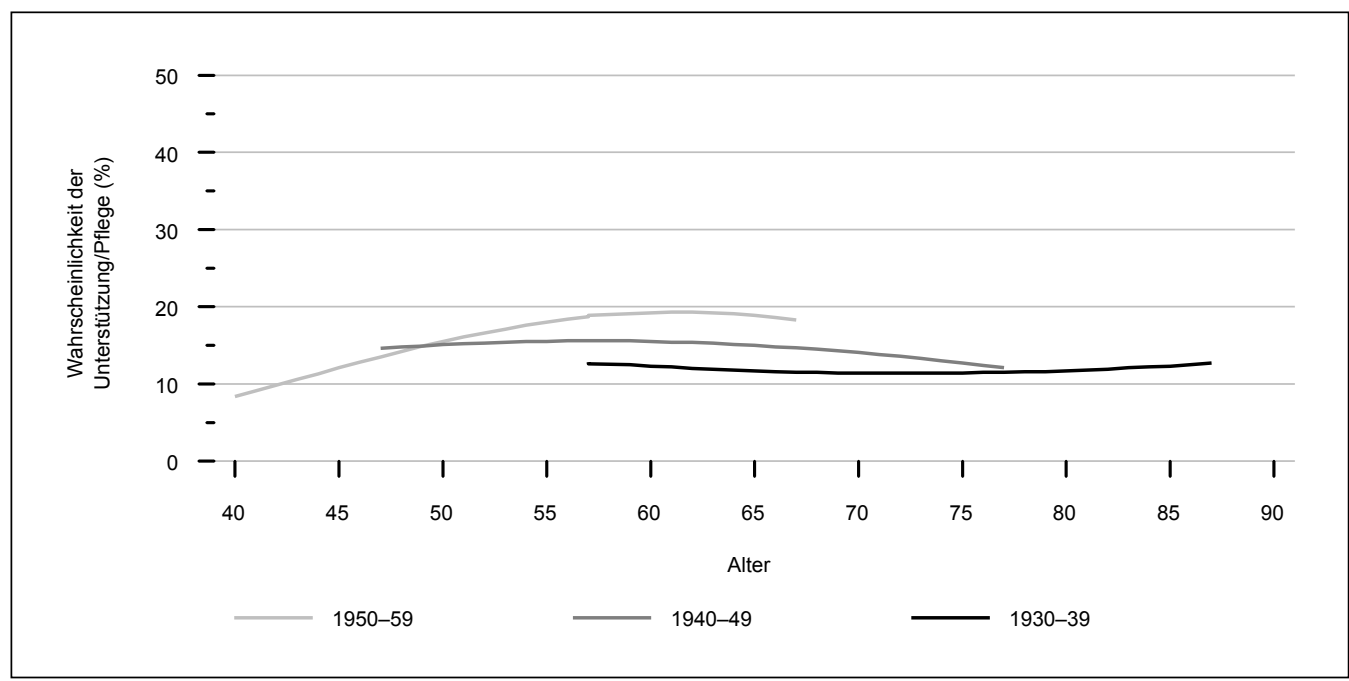

Quelle: DEAS 1996-2017, Schätzungen basieren auf n = 13998 Studienteilnehmenden mit insgesamt n = 29186 Beobachtungen, kontrolliert für Region und Geschlecht.

Für die Kohorte der 1930 bis 1939 Geborenen zeigt sich im Beobachtungszeitraum ein leichter Rückgang der Wahrscheinlichkeit Unterstützung und/oder Pflege zu leisten von 13 Prozent (im Alter von 57 Jahren) auf elf Prozent (im Alter von 69 Jahren), um danach wieder auf 13 Prozent anzusteigen (im Alter von 87 Jahren). Für die zwischen 1940 und 1949 geborenen Personen ist ein Anstieg von 15 Prozent (im Alter von 47 Jahren) auf 16 Prozent (im Alter von 56 Jahren) zu beobachten, sowie ein anschließender Rückgang auf zwölf Prozent (im Alter von 77 Jahren). Für die jüngste Kohorte (1950 bis 1959) ist ein Anstieg der Wahrscheinlichkeit von acht Prozent (im Alter von 40 Jahren) auf 19 Prozent (im Alter von 61 Jahren) zu beobachten. Bis zum Ende des Beobachtungszeitraums für diese Kohorte (im Alter von 67 Jahren) geht dieser Wert auf 18 Prozent zurück. Sowohl Unterschiede in der Wahrscheinlichkeit im Alter von 6o Jahren wie Unterschiede im geschätzten Verlauf der Wahrscheinlichkeit erweisen sich über die betrachteten Kohorten hinweg als signifikant.

Trotz des gesellschaftlichen Wandels in der Unterstützungs- und Pflegetätigkeit gibt es keine Hinweise, dass sich damit auch die geschlechtsspezifischen Muster verändern würden (Abbildung 6-6): Über alle drei Geburtskohorten hinweg bleiben die Unterschiede zwischen Frauen und Männern erhalten: Die Alternsverläufe beider Geschlechter verlaufen ungefähr parallel zueinander mit einer durchweg höheren Wahrscheinlichkeit für Frauen, Verwandte oder nicht-verwandte Personen zu unterstützen und/oder zu pflegen. Demnach zeichnet sich in diesem Bereich der Sorgetätigkeiten, im Gegensatz zur Enkelbetreuung, bislang keine Annäherung der Geschlechter ab.
Der zeitliche Umfang geleisteter Unterstützungsund Pflegeleistungen steigt im Verlauf der zweiten Lebenshälfte an.

Eine ergänzende Betrachtung des zeitlichen Umfangs der geleisteten Unterstützung und Pflege legt offen, dass sich die Intensität der erbrachten Leistungen umgekehrt $\mathrm{zu}$ ihrer Wahrscheinlichkeit entwickelt (Abbildung 6-7): In dem Alter, in dem diese Sorgetätigkeit am häufigsten übernommen wird, fällt ihr Umfang am geringsten aus. Bis Ende 50 - das Alter also, in der die Unterstützungswahrscheinlichkeit ihren Höhenpunkt erreicht - bewegt sich die Intensität auf ihrem geringsten Niveau von kaum mehr als zehn Stunden pro Woche. Von da 
Abbildung 6-6 Wahrscheinlichkeit gesundheitsbedingter Unterstützung und Pflege bei Frauen und Männern nach Kohorten im Alternsverlauf

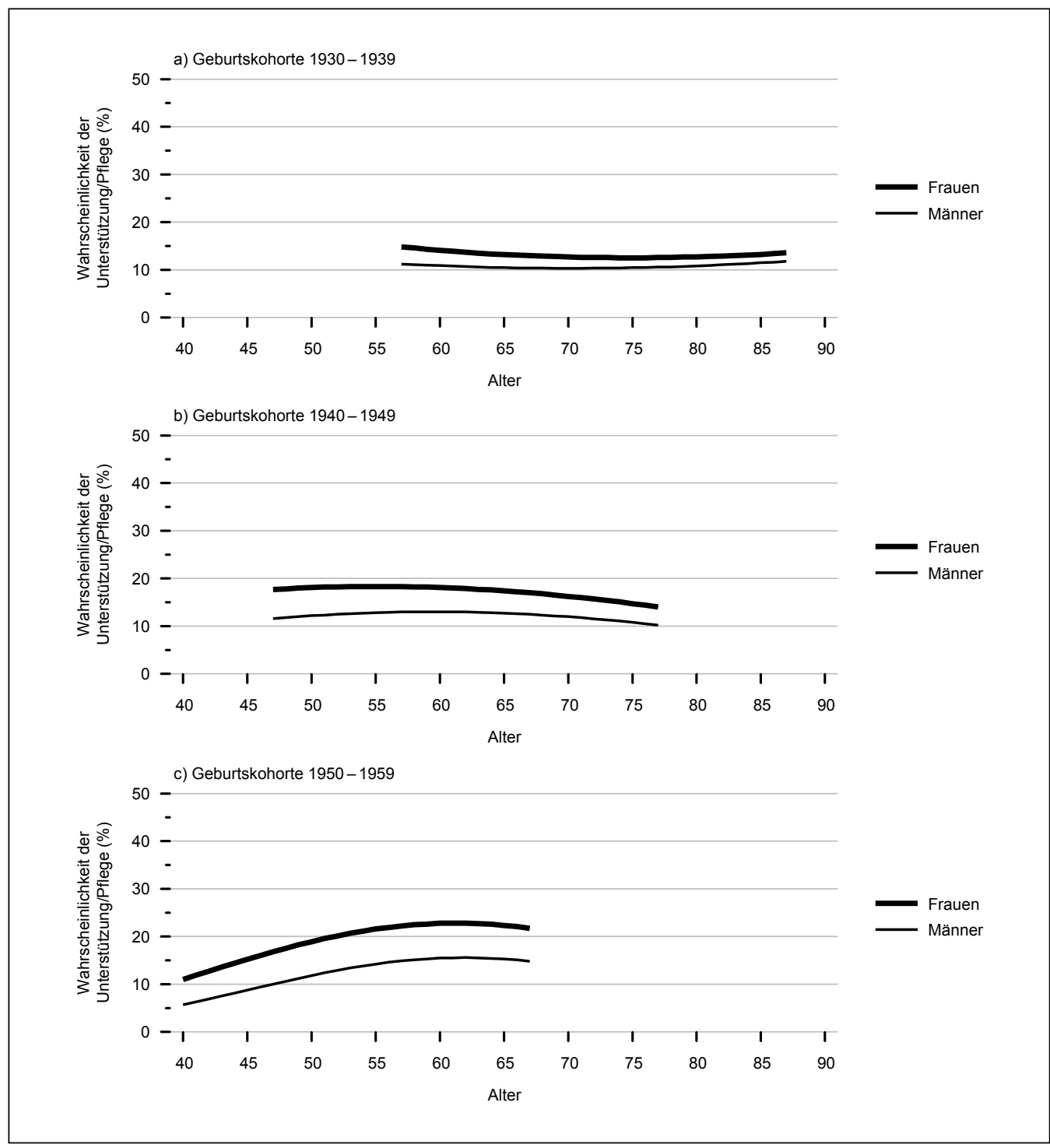

Quelle: DEAS 1996-2017, Schätzungen basieren auf $n=13998$ Studienteilnehmenden mit insgesamt $n=29186$ Beobachtungen, kontrolliert für Region.

In der Kohorte der 1930 bis 1939 Geborenen liegt die Wahrscheinlichkeit, Unterstützung und/oder Pflege zu leisten, im Alter von 60 Jahren bei 14 Prozent bei den Frauen und bei elf Prozent bei den Männern. In der mittleren Kohorte (1940 bis 1949) beträgt die vergleichbare Wahrscheinlichkeit 18 Prozent bei den Frauen und ${ }_{13}$ Prozent bei den Männern. In der Kohorte der 1950 bis 1959 Geborenen beträgt sie 23 Prozent bei den Frauen und 16 Prozent bei den Männern. Die geschätzten Geschlechterunterschiede der Wahrscheinlichkeiten ändern sich weder in ihrem Niveau noch im Alternsverlauf über die Kohorten hinweg. 
an beginnt sie jedoch sichtlich anzuwachsen und erreicht im Alter von 79 Jahren einen doppelt so hohen Umfang: In diesem Alter üben Personen, die mindestens eine andere Person unterstützen und/oder pflegen, diese Tätigkeit im Durchschnitt 20 Wochenstunden aus. Danach steigt sie weiter über den betrachteten Zeitraum auf 27 Stunden im Alter von 90 Jahren.

Dabei fällt der Anstieg im Alternsverlauf bei den Männern deutlicher aus als bei den Frauen (Abbildung 6-7). Allerdings erweisen sich diese Geschlechterunterschiede nur in der Altersphase als statistisch bedeutsam, in der diese Tätigkeit am häufigsten übernommen wird: zwischen dem 47. und 66. Lebensjahr. Für diese Altersspanne zeigt sich also nicht nur, dass Frauen dieser Sorgetätigkeit häufiger nachgehen, sondern dies auch mit größerer Intensität tun: Im Alter von 60 Jahren übernehmen Frauen, die mindestens eine angehörige Person aufgrund deren schlechter $\mathrm{Ge}$ sundheit unterstützen, betreuen und/oder pflegen, diese Aufgabe durchschnittlich 13 Wochenstunden. Männer, die diese Sorgetätigkeit ausüben, tun das in diesem Alter im Umfang von zehn Stunden pro Woche. Der geschlechtsspezifische Alternsverlauf zeigt zunächst ein Anwachsen des Geschlechter-

Abbildung 6-7 Umfang gesundheitsbedingter Unterstützung und Pflege (in Wochenstunden) bei Frauen und Männern im Alternsverlauf

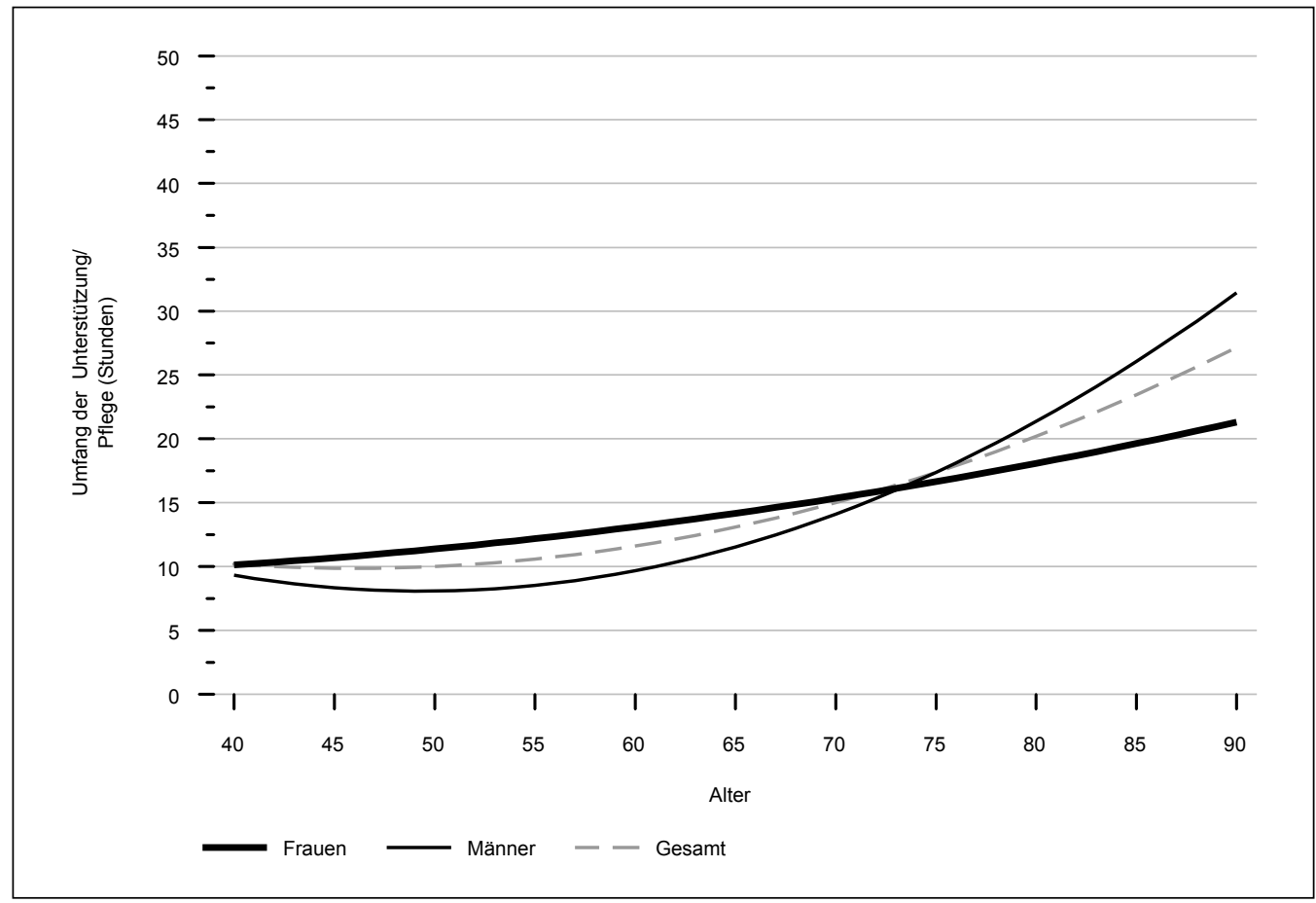

Quelle: DEAS 1996-2017, Schätzungen basieren auf $n=3991$ Studienteilnehmenden mit insgesamt $n=5360$ Beobachtungen, kontrolliert für Region.

Bei Frauen steigt der mittlere Umfang geleisteter Unterstützung und/oder Pflege von zehn Wochenstunden (im Alter von 40 Jahren) auf 13 Wochenstunden an (im Alter von 6o Jahren). Bei Männern steigt der Umfang von neun Wochenstunden (im Alter von 40 Jahren) auf zehn Wochenstunden an (im Alter von 6o Jahren). Im Alter von 6o Jahren unterscheiden sich die geschätzten Umfänge für Frauen und Männer signifikant. Die geschlechtsspezifischen Unterschiede im geschätzten Alternsverlauf sind signifikant. 
unterschiedes bis zum 56. Lebensjahr (Unterschied von vier Wochenstunden). Ab dem 66. Lebensjahr zeigt sich kein Unterschied mehr im zeitlichen Betreuungsaufwand von Frauen und Männern. Die in Abbildung 6-7 sichtbaren Unterschiede im hohen Alter sind - vermutlich aufgrund der vergleichs- weise geringen Befragtenzahlen - statistisch nicht signifikant. Ob sich dieses geschlechtsspezifische (Verlaufs-)Muster über die Geburtskohorten hinweg gewandelt hat, kann - ebenfalls aufgrund der geringen Fallzahlen - nicht untersucht werden.

\subsection{Diskussion}

\section{Zusammenfassung}

Die Ergebnisse belegen deutliche Geschlechterunterschiede in der Ausübung von Sorgetätigkeiten, die sich sowohl im Alter von 60 Jahren als auch im Verlauf der zweiten Lebenshälfte zeigen (erste Forschungsfrage). Darüber hinaus gibt es punktuelle Hinweise auf eine in den später geborenen Kohorten stattfindende Angleichung dieser Unterschiede zwischen Frauen und Männern (zweite Forschungsfrage).

Konkret hat sich gezeigt, dass Frauen nicht nur häufiger Enkelkinder betreuen und hilfsbedürftige Familienangehörige oder Personen aus der Nachbarschaft oder dem Freundes- und Bekanntenkreis unterstützen und pflegen als Männer. Frauen tun dies auch zeitintensiver, früher in der zweiten Lebenshälfte und tendenziell über eine längere Zeitspanne hinweg als Männer. Während bei Frauen diese Aufgaben bereits in der späten Erwerbsphase beginnen, konzentrieren sich diese Tätigkeiten bei den Männern eher auf die Zeit nach dem Renteneintritt. So kommt es mit Beginn der zweiten Lebenshälfte typischerweise zunächst $\mathrm{zu}$ einem Auseinanderdriften der geschlechtsspezifischen Verläufe, das etwa Mitte 50 (Unterstützung und Pflege) und Anfang 60 (Enkelbetreuung) seinen Höhepunkt erreicht. In dieser Altersphase fallen die Unterschiede in der Übernahme von Sorgetätigkeiten zwischen Frauen und Männern am größten aus. Danach setzt eine Angleichung der Unterschiede ein. Dieser Prozess geht allerdings weniger auf ein aktives „Aufholen“ der Männer zurück, sondern ist vielmehr das Resultat eines allgemei- nen altersbedingten Rückgangs, der Frauen stärker betrifft als Männer.

Hinweise für einen gesellschaftlichen Wandel dieser geschlechtsspezifischen Muster und $\mathrm{Al}$ ternsverläufe finden sich bislang nur für die Enkelbetreuung: Für die Kohorte der 1950 bis 1959 Geborenen konnte - im Vergleich zur Kohorte 1930 bis 1939 - eine Angleichung zwischen den Geschlechtern festgestellt werden. Diese Angleichung beruht auf zwei Verschiebungen: Erstens darauf, dass sich Männer späterer Geburtsjahrgänge stärker an der Enkelbetreuung beteiligen als früher geborene Männer und zweitens, dass Frauen der jüngeren Kohorten seltener betreuen. Daneben hat sich für beide Geschlechter gleichermaßen die Hauptphase der Enkelbetreuung in ein deutlich höheres Lebensalter verlagert: Der soziale Wandel zeigt sich in einer Verlagerung von durchschnittlich zehn Jahren zwischen der 1930er und der 1950er Kohorte. Diese biografische Verlagerung hat dazu geführt, dass ein Großteil der Enkelbetreuungen nunmehr im Rentenalter stattfindet.

Eine vergleichbare Annäherung der Geschlechterunterschiede ist für die Unterstützung und Pflege kranker verwandter oder nicht-verwandter Personen nicht zu beobachten: Zwar werden diese Arbeiten in der Kohorte der 1950 bis 1959 Geborenen häufiger übernommen als in der Kohorte der 1930 bis 1939 Geborenen und es ist zu einer stärkeren Konzentration auf eine bestimmte Altersphase - um das 60. Lebensalter herum - gekommen. Doch die Unterschiede zwischen den Geschlechtern bestehen nach wie vor. 


\section{Interpretation}

Anhand der Untersuchung alterstypischer Sorgetätigkeiten bestätigt sich auch für das höhere Lebensalter und insbesondere die späte Erwerbsphase die bekannte klassische geschlechtsspezifische Rollen- und Arbeitsverteilung: Familienbezogene Arbeiten liegen - wahrscheinlich in Fortführung der in vorangegangenen Lebens- und Familienphasen praktizierten Arbeitsteilung - auch in diesem Alter eher im Verantwortungsbereich der Frauen. Frauen pflegen familiale und andere enge Beziehungen stärker, indem sie häufiger, zeitintensiver und - wie die Längsschnittanalysen in diesem Band besonders eindrücklich zeigen - dauerhafter als gleichaltrige Männer Unterstützung, Betreuung bis hin zu krankheitsverursachter Pflege im privaten Umfeld leisten.

Dass sich bei Frauen eine Häufung der Betreuungs- und Unterstützungsleistungen in der späten Erwerbsphase zeigt, während sich Männer erst später (zumeist im frühen Rentenalter) verstärkt beteiligen, hat einerseits demografische Ursachen: Dass Frauen in einem jüngeren Alter Enkelbetreuung aufnehmen als Männer ist teilweise auf ihr geringeres Alter bei Geburt des ersten Kindes zurückzuführen sowie den typischen Altersabstand in Ehen und Partnerschaften von etwa drei Jahren. Männer sind bei Geburt ihres ersten Enkelkindes im Schnitt drei Jahre älter als Frauen (54 Jahre zu 51 Jahre, vgl. Mahne \& Klaus 2017: 236). Die generell seltenere Enkelbetreuungsübernahme unter Männern hat möglicherweise auch damit zu tun, dass Männer etwas häufiger kinderlos sind und damit auch häufiger als Frauen keine Enkelkinder haben. So haben elf Prozent der 70- bis 85-jährigen Frauen keine Enkelkinder im Vergleich zu 18 Prozent der Männer (Deutscher Alterssurvey 2014, eigene Auswertungen). Das grundsätzlich stärkere Engagement von Frauen in den beiden betrachteten Sorgebereichen ist zugleich Ausdruck geschlechtsspezifischer Muster der Aufteilung von bezahlter Erwerbs- und unbezahlter Sorgetätigkeit. Die über Jahrzehnte hinweg bestehende geringere Erwerbsbeteiligung der Frauen, die sich trotz stei- gender Tendenz auch nach wie vor in der späten Erwerbsphase zeigt, forciert geschlechtsspezifische Erwartungsmuster: Danach sind sie es, die sich stärker im Sorgebereich engagieren ebenso wie erwerbstätige Frauen eine bestehende Erwerbstätigkeit im Fall nachgefragter Betreuungs- oder Pflegeaufgaben eher reduzieren oder auch ganz aufgeben als Männer.

Auch die beschriebenen gesellschaftlichen Veränderungen im Verlauf der letzten Jahre sind zunächst Folge demografischer Verschiebungen: So geht der stattgefundene Aufschub der Enkelbetreuung in ein biografisch höheres Alter - zumindest partiell - direkt darauf zurück, dass heutige Eltern später Großeltern werden als noch vor einigen Jahren (Klaus \& Mahne 2017: 235). Das wiederum ist Folge der späteren Familiengründung. Allerdings kann die Abnahme der Geschlechterunterschiede in der Enkelkinderbetreuung auch als Ausdruck einer einsetzenden Aufweichung der geschlechtsspezifischen Aufgaben- und Rollenteilung interpretiert werden: Denn Frauen in den später geborenen Kohorten sind eben auch zu höheren Anteilen erwerbstätig als dies Frauen in den früher geborenen Kohorten waren. Parallel dazu engagieren sich Männer in den später geborenen Kohorten eher in der Enkelbetreuung, was bei ihren eigenen Vätern meist noch deutlich seltener der Fall war.

Sehr deutlich schlägt sich der demografische Wandel - insbesondere die zunehmende Hochaltrigkeit - in einem Wandel von Unterstützungsund Pflegetätigkeiten über die Generationen hinweg nieder: So ist die Wahrscheinlichkeit, die Tätigkeiten auszuüben, in den später geborenen Kohorten angestiegen ebenso wie es zu einer Verschiebung in ein höheres Alter gekommen ist. Da gerade unter den Ältesten der Anteil der Verwitweten zurückgegangen ist beziehungsweise sich die Verwitwung in ein höheres Alter verschoben hat (Engstler \& Klaus 2017: 205), ist davon auszugehen, dass die typischerweise im höheren Alter ausgeübte Pflege der Partnerin oder des Partners zugewonnen hat - indem sie stärker nachgefragt wird, aber auch aufgrund länger erhaltener funktionaler Gesundheit eher geleistet werden kann. 
Allerdings ist hier bei später geborenen Kohorten keine Angleichung zwischen Frauen und Männern festzustellen.

\section{Politische Implikationen}

Nicht zuletzt begründet durch die nach wie vor bestehenden und offenbar auch beständigen $\mathrm{Ge}$ schlechterunterschiede in der Übernahme verschiedener Sorgetätigkeiten, besteht weiterhin dringender politischer Handlungsbedarf, um einer einseitigen Be- oder gar Überlastung von Frauen entgegenzuwirken. Ein Ziel ist die Entlastung von Frauen aber auch von Männern, die im hohen (Erwerbs-)Alter Sorgetätigkeiten übernehmen (wollen) und möglicherweise zugleich erwerbstätig sind (sein wollen).

Um das zu erreichen, sind in erster Linie bereits bestehende Maßnahmen und Vorhaben weiter voranzutreiben, die auf den Ausbau institutioneller Betreuungseinrichtungen (in den Bereichen Kinder- und Altenversorgung) und die Ausweitung des Angebots professioneller Dienstleister zielen. Deren Verfügbarkeit kann in ergänzender Form dazu beitragen, dass der Umfang der informell übernommenen Sorgetätigkeiten besser auf das jeweils leistbare oder gewollte Maß zugeschnitten werden kann. Oder aber institutionelle oder professionelle Betreuungsmöglichkeiten springen gänzlich ein, wenn anstehende Sorgeaufgaben - aus welchen Gründen auch immer - (temporär) nicht von den 40 - bis 90 -Jährigen privat übernommen werden können.

Darüber hinaus sind, nicht nur unter der Prämisse der Entlastung der Frauen, sondern auch im Sinne einer Geschlechtergerechtigkeit, Männer stärker für relevante Sorgebereiche zu gewinnen. Auf diese Weise lassen sich nachgefragte Betreuungs- und Pflegeaufgaben im privaten Umfeld auf mehrere Schultern verteilen und so das Risiko der Überlastung einzelner Hauptverantwortlicher mindern. Eine verstärkte Einbindung von Personen aus der Nachbarschaft und aus dem Freundes- und Bekanntenkreis könnte ebenfalls zur Ent- lastung beitragen, allerdings muss auch hier der Aspekt der Geschlechtergerechtigkeit im Blick behalten werden. Ohne politische Gegensteuerung würde die bei Frauen höhere Wahrscheinlichkeit der Übernahme von Pflege und Betreuung im Freundeskreis und in der Nachbarschaft wiederum die traditionelle Arbeitsteilung zementieren und die Doppelbelastungen für Frauen dauerhaft im Verlauf der zweiten Lebenshälfte verschärfen. Grundlegend für mehr Gleichberechtigung im Bereich der gesellschaftlich höchst relevanten Sorgearbeit ist es, weiter an der Vermittlung und Etablierung modernisierter Rollenbilder zu arbeiten - bereits in frühen Lebens- und Familienphasen und nicht zuletzt unterstützt durch eine weitere Förderung der Frauenerwerbsbeteiligung.

Parallel dazu sind nach wie vor Bedingungen zu schaffen und zu verbessern, die es erwerbstätigen Frauen und Männern gleichermaßen ermöglichen, Sorgeaufgaben zu übernehmen. Neben der Gewährleistung von Arbeitsplatzsicherheit und finanzieller Absicherung in Phasen temporärer Erwerbsreduktion steht hier vor allem eine Flexibilisierung von Arbeitszeiten und -ort im Vordergrund. Denn ein Vereinbarkeitsproblem besteht selten allein aufgrund der benötigten Zeit für Sorgetätigkeiten, sondern auch aufgrund der Tatsache, dass Betreuungs- und Pflegeverpflichtungen zeitlich nicht gut planbar sind und oft kurzfristiges Handeln erfordern. Zudem darf neben den Ansprüchen der bezahlten Arbeit auch der Stress und die z.B. emotional teils hohe Belastung durch unbezahlte Arbeit nicht vergessen werden. Auch in diesem Zusammenhang sind Unterstützungs- und Entlastungmaßnahmen für stark beanspruchte Sorgeleistende zu konzipieren.

Politische Maßnahmen, die etwa eine Übernahme von Enkelkinderbetreuung durch die Großeltern unterstützen, sollten umgekehrt auch jeweils daraufhin geprüft werden, wie gegebenenfalls eine Erwerbstätigkeit der Großeltern dadurch beeinflusst ist. Aufgrund der insgesamt geringeren Rentenanwartschaften von Frauen wäre es aus unserer Sicht ein falsches Signal, Großmütter zu ermutigen, ihre Erwerbstätigkeit zugunsten der En- 
kelkinderbetreuung frühzeitig zu beenden oder zu reduzieren, mit den entsprechenden nachteiligen Konsequenzen für die individuellen Alterseinkommen. Auch der derzeit oft beklagte Arbeitskräftemangel, bei gleichzeitigem Trend zum längeren Arbeiten für bestimmte Gruppen, sollte nicht durch Anreize zur Beendigung der beruflichen Tätigkeit aufgrund der Übernahme familienbezogener Verpflichtungen verschärft werden. Vor dem Hintergrund der Tatsache, dass viele Großeltern die Enkelkinderbetreuung übernehmen, um ihre erwachsenen Kinder zu entlasten und ihnen für die berufliche Karriere „den Rücken freizuhalten“, geht es auch immer darum, durch staatliche Infrastruktur Betreuungsarrangements zu schaffen, die allen Generationen die Arbeitsmarktpartizipation ermöglichen. Vergleichbar zu den Maßnahmen, Väter stärker zur Übernahme von Erziehungsaufgaben zu motivieren, ist auch hier zu überlegen, die Enkelkinderbetreuung auf mehr Personen zu verteilen. Zentral ist dabei, dass Maßnahmen zur Vereinbarkeit von Großelternschaft und Berufstätigkeit insbesondere auch Großväter erreichen, die sich dann biografisch früher an der Enkelkin- derbetreuung beteiligen. Dies wäre insbesondere vor dem Hintergrund sich langsam wandelnder Geschlechternormen und modernisierter Rollenbilder erfolgversprechend.

Insgesamt ist es aus unserer Sicht von großer Bedeutung, dass politische Maßnahmen zur Verbesserung der Vereinbarkeit von unbezahlten familialen Sorgetätigkeiten und bezahlten Erwerbstätigkeiten nicht verkürzt auf jene Personen zugeschnitten werden, die derzeit mehrheitlich vor der Frage der Vereinbarkeit stehen. Das heißt, entsprechende Maßnahmen und Unterstützungsangebote sind grundsätzlich an alle Personen zu adressieren und nicht nur an Frauen. Von verbesserten Rahmenbedingungen sollten erwerbstätige Männer, die Angehörige betreuen und pflegen, ebenso profitieren wie erwerbstätige Frauen. Ohne politische Steuerung ist eine Gleichstellung der Geschlechter in Bezug auf familienbezogene Aufgaben und Sorgetätigkeiten in Deutschland jedenfalls kaum zu erreichen. Und bislang gestaltet sich in diesem Bereich die Situation späterer Jahrgänge kaum besser als die der früheren Jahrgänge von Frauen und Männern.

\section{Literatur}

Bestmann, B., Wüstholz, E., \& Verheyen, F. (2014). Pflegen: Belastung und sozialer Zusammenhalt. Eine Befragung zur Situation von pflegenden Angehörigen [WINEG Wissen 4]. Hamburg: Techniker Krankenkasse.

Bundesministerium für Familie, Senioren, Frauen und Jugend (2018). Kindertagesbetreuung Kompakt. Ausbaustand und Bedarf 2017. Berlin: Bundesministerium für Familie, Senioren, Frauen und Jugend.

Bolin, K., Lindgren, B., \& Lundborg, P. (2008). Informal and formal care among single living elderly in Europe. Health economics, 17(3), 393-409.

Brandt, M., Haberkern, K., \& Szydlik, M. (2009). Intergenerational help and care in Europe. European Sociological Review, 25(5), 585-601.

Brettschneider, A., \& Klammer, U. (2016). Lebenswege in die Altersarmut. Biografische Analysen und sozialpolitische Perspektiven. Berlin: Duncker \& Humblot.

Brüderl, J. (2010). Kausalanalyse mit Paneldaten. In: C. Wolf \& H. Best (Hrsg.) Handbuch der sozialwissenschaftlichen Datenanalyse (S. 963-994). Wiesbaden: VS Verlag für Sozialwissenschaften.

Bundesministerium für Familie, Senioren, Frauen und Jugend (2018). Kindertagesbetreuung Kompakt. Ausbaustand und Bedarf 2017. Berlin: Bundesministerium für Familie, Senioren, Frauen und Jugend. 
Deindl, C., \& Brandt, M. (2011). Financial support and practical help between older parents and their middle-aged children in Europe. Ageing \& Society, 31(04), 645-662.

Deutscher Bundestag (2017). Zweiter Gleichstellungsbericht der Bundesregierung. Erwerbs- und Sorgearbeit gemeinsam neu gestalten. (Drucksache 18/12840). Berlin: Deutscher Bundestag.

Engstler, H., \& Klaus, D. (2017). Auslaufmodell ,traditionelle Ehe'? Wandel der Lebensformen und der Arbeitsteilung von Paaren in der zweiten Lebenshälfte. In: K. Mahne, J. K. Wolff, J. Simonson \& C. TeschRömer (Hrsg.) Altern im Wandel. Zwei Jahrzehnte Deutscher Alterssurvey (DEAS) (S. 201-213). Wiesbaden: Springer VS.

Engstler, H., \& Tesch-Römer, C. (2017). Zeitverwendung von Erwachsenen, die ein Haushaltsmitglied pflegen. In: Statistisches Bundesamt (Hrsg.) Wie die Zeit vergeht: Analysen zur Zeitverwendung in Deutschland. Beiträge zur Ergebniskonferenz der Zeitverwendungserhebung 2012/2013 am 5./6. Oktober 2016 in Wiesbaden (S. 229-244). Wiesbaden: Statistisches Bundesamt.

Frerk, T., \& Leitner, S. (2017). Zur Vereinbarkeit von Pflege und Beruf: Quantitative und qualitative Perspektiven. Sozialer Fortschritt, 66(3-4), 267-283. doi: 10.3790/sfo.66.3-4.267.

Geyer, J. (2016). Informell Pflegende in der deutschen Erwerbsbevölkerung: Soziodemografie, Pflegesituation und Erwerbsverhalten. In: Zentrum für Qualität in der Pflege (Hrsg.) ZQP-Themenreport. Vereinbarkeit von Beruf und Pflege (S. 24-43). Berlin: Zentrum für Qualität in der Pflege.

Geyer, J., \& Schulz, E. (2014). Who cares ? Die Bedeutung der informellen Pflege durch Erwerbstätige in Deutschland. DIW Wochenbericht, 81(14), 294-301.

Hank, K., \& Buber, I. (2009). Grandparents caring for their grandchildren. Findings From the 2004 Survey of Health, Ageing, and Retirement in Europe. Journal of Family Issues, 30(1), 53-73.

Henz, U. (2004). The effects of informal care on paid-work participation in Great Britain: a lifecourse perspective. Ageing \& Society, 24(06), 851-880.

Hobler, D., Klenner, C., Pfahl, S., Sopp, P., \& Wagner, A. (2017). Wer leistet unbezahlte Arbeit ? Hausarbeit, Kindererziehung und Pflege im Geschlechtervergleich. Aktuelle Auswertungen aus dem WSI GenderDatenPortal [WSI Report Nr. 35]. Düsseldorf: Wirtschafts- und Sozialwissenschaftliches Institut (WSI) in der Hans-Böckler-Stiftung.

Igel, C., \& Szydlik, M. (2011). Grandchild care and welfare state arrangements in Europe. Journal of European Social Policy, 21(3), 210-224. doi: 10.1177/0958928711401766.

Kalmijn, M., \& Saraceno, C. (2008). A comparative perspective on intergenerational support. European Societies, $10(3), 479-508$.

Keck, W. (2016). Was kommt nach der Pflege? Die Pflege eines Angehörigen senkt Beschäftigungschancen von Pflegepersonen nachhaltig. Sozialer Fortschritt, 65(5), 112-119.

Keck, W. (2012). Die Vereinbarkeit von häuslicher Pflege und Beruf. Bern: Huber. doi: 10.3790/sfo.65.5.112.

Klaus, D. (2009). Why Do Adult Children Support Their Parents? Journal of Comparative Family Studies, $4 O(2), 227-241$.

Klaus, D., \& Tesch-Römer, C. (2017). Pflege und Unterstützung bei gesundheitlichen Einschränkungen: Welchen Beitrag leisten Personen in der zweiten Lebenshälfte für andere ? In: K. Mahne, J. K. Wolff, J. Simonson \& C. Tesch-Römer (Hrsg.) Altern im Wandel. Zwei Jahrzehnte Deutscher Alterssurvey (DEAS) (S. 185-200). Wiesbaden: Springer VS.

Leopold, T., \& Raab, M. (2011). Kurzzeitreziprozität in späten Eltern-Kind-Beziehungen. In: P. A. Berg (Hrsg.) Reproduktion von Ungleichheit durch Arbeit und Familie (S. 93-119). Wiesbaden: VS Verlag für Sozialwissenschaften.

Leopold, T., Raab, M., \& Engelhardt, H. (2014). The transition to parent care: Costs, commitments, and caregiver selection among children. Journal of Marriage and Family, 76(2), 300-318. 
Leopold, T., \& Skopek, J. (2015). The Delay of Grandparenthood: A Cohort Comparison in East and West Germany. Journal of Marriage and Family, 77(2), 441-460.

Mahne, K., \& Klaus, D. (2017). Zwischen Enkelglück und (Groß-)Elternpflicht - die Bedeutung und Ausgestaltung von Beziehungen zwischen Großeltern und Enkelkindern. In: K. Mahne, J. K. Wolff, J. Simonson \& C. Tesch-Römer (Hrsg.) Altern im Wandel. Zwei Jahrzehnte Deutscher Alterssurvey (DEAS) (S. 231-245). Wiesbaden: Springer VS.

Nowossadeck, S., Engstler, H., \& Klaus, D. (2016). Pflege und Unterstützung durch Angehörige. [Report Altersdaten 1/2016]. Berlin: Deutsches Zentrum für Altersfragen.

Rothgang, H., Iwansky, S., Müller, R., Sauer, S., \& Unger, R. (2010). Barmer GEK Pflegereport 2010. Schwerpunktthema: Demenz und Pflege [Schriftenreihe zur Gesundheitsanalyse Band 5]. Schwäbisch Gmünd: BARMER GEK.

Runde, P., Giese, R., Kaphengst, C., \& Hess, J. (2009). AOK-Trendbericht Pflege II. Entwicklung in der häuslichen Pflege seit Einführung der Pflegeversicherung. Hamburg: Universität Hamburg, Fakultät Wirtschafts- und Sozialwissenschaften.

Schäufele, M., Köhler, L., \& Hendlmeier, I. (2016). Erwerbstätigkeit und Pflege von Menschen mit Demenz: Ergebnisse einer bundesweiten Repräsentativstudie. In: Zentrum für Qualität in der Pflege (Hrsg.) Vereinbarkeit von Beruf und Pflege (S. 44-59). Berlin: Zentrum für Qualität in der Pflege.

Schröder, A. (2007). Prinzipien der Panelanalyse. In: S. Albers, D. Klapper, U. Konradt, A. Walter \& J. Wolf (Hrsg.) Methodik der empirischen Forschung (S. 261-276). Wiesbaden: Springer Gabler.

Schwarz, N., \& Schwahn, F. (2016). Entwicklung der unbezahlten Arbeit privater Haushalte. Bewertung und Vergleich mit gesamtwirtschaftlichen Größen. WISTA - Wirtschaft und Statistik, 2016(2), 35-51.

Schwinger, A., Tsiasioti, C., \& Klauber, J. (2016). Unterstützungsbedarf in der informellen Pflege - eine Befragung pflegender Angehöriger. In: K. Jacobs, A. Kuhlmey, S. Greß, J. Klauber \& A. Schwinger (Hrsg.) Pflege-Report 2016 (S. 189-216). Stuttgart: Schattauer.

Spiess, C. K., \& Schneider, A. U. (2003). Interactions between care-giving and paid work hours among European midlife women, 1994 to 1996. Ageing \& Society, 23(1), 41-68.

Statistisches Bundesamt (2018). Statistiken der Kinder- und Jugendhilfe. Kinder und tätige Personen in Tageseinrichtungen und in öffentlich geförderter Kindertagespflege am 01. 03. 2017. Wiesbaden: Statistisches Bundesamt.

Statistisches Bundesamt (2017). Pflegestatistik 2015. Wiesbaden: Statistisches Bundesamt.

Stuifbergen, M. C., Delden, J. J. M. van, \& Dykstra, P. A. (2008). The implications of today's family structures for support giving to older parents. Ageing \& Society, 28(3), 413-434.

TNS Infratest Sozialforschung (2011). Abschlussbericht zur Studie „Wirkungen des Pflege-Weiterentwicklungsgesetzes". Berlin: Bundesministerium für Gesundheit.

Vogel, C., \& Künemund, H. (2018). Armut im Alter. In: P. Böhnke, J. Dittmann \& J. Goebel (Hrsg.) Handbuch Armut (S. 144-153). Leverkusen: Barbara Budrich.

Vogel, C., \& Scherger, S. (2018). Einleitung. In: C. Vogel \& S. Scherger (Hrsg.) Arbeit im Alter. Zur Bedeutung bezahlter und unbezahlter Tätigkeiten in der Lebensphase Ruhestand (S.1-25). Wiesbaden: Springer VS. 
Open Access Dieses Kapitel wird unter der Creative Commons Namensnennung 4.0 International Lizenz (http:// creativecommons.org/licenses/by/4.0/deed.de) veröffentlicht, welche die Nutzung, Vervielfältigung, Bearbeitung, Verbreitung und Wiedergabe in jeglichem Medium und Format erlaubt, sofern Sie den/die ursprünglichen Autor(en) und die Quelle ordnungsgemäß nennen, einen Link zur Creative Commons Lizenz beifügen und angeben, ob Änderungen vorgenommen wurden.

Die in diesem Kapitel enthaltenen Bilder und sonstiges Drittmaterial unterliegen ebenfalls der genannten Creative Commons Lizenz, sofern sich aus der Abbildungslegende nichts anderes ergibt. Sofern das betreffende Material nicht unter der genannten Creative Commons Lizenz steht und die betreffende Handlung nicht nach gesetzlichen Vorschriften erlaubt ist, ist für die oben aufgeführten Weiterverwendungen des Materials die Einwilligung des jeweiligen Rechteinhabers einzuholen. 


\title{
7. Ehrenamtliches Engagement von Frauen und Männern im Verlauf der zweiten Lebenshälfte
}

\author{
Claudia Vogel und Laura Romeu Gordo
}

\section{Kernaussagen}

Für Frauen und Männer steigt die Wahrscheinlichkeit, sich in formalen Organisationen ehrenamtlich zu engagieren, zunächst an, sinkt dann mit zunehmendem Alter jedoch wieder: Im Alter von 40 bis 58 Jahren wird es wahrscheinlicher, sich ehrenamtlich zu engagieren. Ab 59 Jahren wird es mit steigendem Alter unwahrscheinlicher, ehrenamtlich engagiert zu sein. Am wahrscheinlichsten ist die ehrenamtliche Partizipation im Alter zwischen 53 und 63 Jahren, in dieser Lebensphase liegt sie über 19 Prozent.

Über den gesamten Verlauf der zweiten Lebenshälfte haben Männer eine höhere Wahrscheinlichkeit, sich ehrenamtlich zu engagieren, als Frauen: Diese Geschlechterunterschiede sind zu Beginn der zweiten Lebenshälfte größer als im höheren Alter. Das heißt, die Geschlechterunterschiede verringern sich mit steigendem Alter insbesondere in der Ruhestandsphase.

Die Wahrscheinlichkeit für Frauen und Männer, ehrenamtlich engagiert zu sein, ist für später geborene Kohorten über den Alternsverlauf von 40 bis 90 Jahren höher als für früher geborene. Die Geschlechterunterschiede im Ehrenamt bleiben in allen Kohorten bestehen: Während die Kohorten einen ähnlichen Alternsverlauf aufweisen, scheint sich der Wendepunkt, ab dem die Ehrenamtswahrscheinlichkeit mit steigendem Alter sinkt, nach hinten zu verschieben. Dadurch, dass Frauen ähnliche Entwicklungen im Ehrenamt über die Kohorten hinweg aufweisen wie Männer, ist bislang keine Verringerung des Geschlechterunterschieds für später geborene Kohorten zu beobachten.

Die Wahrscheinlichkeiten der Neuaufnahme und Beendigung von ehrenamtlichem Engagement unterscheiden sich im Alternsverlauf, es gibt jedoch kaum Geschlechterunterschiede: Die Wahrscheinlichkeit, eine ehrenamtliche Tätigkeit neu aufzunehmen, nimmt bis zu einem Alter von 54 Jahren zu, und sie sinkt dann mit dem Älterwerden ab. Die Beendigung von Ehrenamt bleibt hingegen im Alternsverlauf stabil. Die stetig geringere Wahrscheinlichkeit, im höheren Alter einer ehrenamtlichen Tätigkeit nachzugehen, ergibt sich daraus, dass in diesem höheren Lebensalter zunehmend weniger Personen ein Ehrenamt neu anfangen und gleichzeitig nicht weniger Engagierte ihre ehrenamtlichen Tätigkeiten beenden. Prozesse der Aufnahme von Ehrenämtern unterscheiden sich nicht signifikant nach Geschlecht und Beendigungsprozesse unterscheiden sich nur minimal nach Geschlecht. 


\subsection{Einleitung}

Viele Ältere sind heute trotz beruflichen Ruhestandes häufig aktiv und übernehmen eine Vielzahl von produktiven Tätigkeiten in der Familie, der Nachbarschaft und der Zivilgesellschaft. Dies können Hausarbeit, Enkelkinderbetreuung, Pflegetätigkeiten, Alltagsunterstützung oder Ehrenämter sein. Solche Tätigkeiten sind für die Wohlfahrtsproduktion und die Lebensqualität älterer Menschen in Deutschland sehr bedeutsam (siehe Kapitel 6 Unbezahlte Sorgetätigkeiten in diesem Band). Ein ehrenamtliches Engagement in einem Verein oder einer Organisation bringt für die Engagierten vielerlei Vorteile mit sich. Es kann sinnstiftend sein und das Gefühl vermitteln, gebraucht zu werden und mitbestimmen zu können. Es kann zudem Freude bereiten und den Alltag strukturieren. Auch geht ehrenamtliches Engagement mit der Knüpfung und Pflege sozialer Kontakte einher, sowie mit der Möglichkeit, die eigenen Erfahrungen und Kenntnisse einzubringen und neue Kenntnisse und Fertigkeiten zu erwerben. Ein Ehrenamt ermöglicht ferner sozial teilzuhaben, und es bringt auch Anerkennung mit sich - gerade mit steigendem Alter, wenn der Beruf in den Hintergrund gerät, sind dies keine unwichtigen Aspekte. Belegt ist darüber hinaus, dass Personen, die sich engagieren, zufriedener und gesünder sind als Personen, die sich nicht ehrenamtlich oder freiwillig engagieren (zum Forschungsstand vgl. Simonson \& Vogel 2018/im Erscheinen).

Wie entwickelt sich das ehrenamtliche Engagement von Menschen, wenn sie das mittlere Lebensalter erreicht haben und älter werden? Aus Querschnittsvergleichen der Anteile Engagierter lässt sich ablesen, dass Frauen und Männer in den Altersgruppen von 40 bis 69 Jahren überdurchschnittlich häufig engagiert sind. Im Alter ab 70 Jahren sind die Anteile Engagierter geringer (und ab 75 Jahre deutlich geringer) (vgl. Vogel, Hagen, Simonson, \& Tesch-Römer 2017: 104). Sind im höheren Alter nur Menschen engagiert, die ein früher im Lebenslauf begonnenes Engagement bis ins Alter fortsetzen oder planen auch ältere Men- schen, die bislang nicht engagiert waren, ein Ehrenamt aufzunehmen? Befunde des Deutschen Alterssurveys (DEAS) 2014 belegen, dass Menschen auch in der zweiten Lebenshälfte noch ehrenamtliche Tätigkeiten neu aufnehmen, denn von den gut 22 Prozent der im Jahr 2014 ehrenamtlich engagierten Personen im Alter von 40 bis 85 Jahren hat „ein Drittel seit 2009 ein (zusätzliches) Engagement begonnen" (vgl. Wetzel \& Simonson 2017: 90). Laut Ergebnissen des Deutschen Freiwilligensurveys haben sich lediglich 35 Prozent der Personen über 64 Jahren rückblickend über ihren gesamten Lebenslauf nie engagiert (Müller \& Tesch-Römer 2017: 164).

Gleichzeitig beenden viele Personen ihr ehrenamtliches Engagement im höheren Alter. Gründe hierfür liegen zum einen bei den ehrenamtlichen Tätigkeiten selbst. Entweder war eine solche Tätigkeit von vornherein zeitlich begrenzt, was rund die Hälfte der 65-Jährigen und Älteren als Beendigungsgrund angibt, oder aber der zeitliche Aufwand durch das Engagement wurde als zu hoch empfunden (dies trifft auf rund ein Drittel der 65-Jährigen und Älteren zu, vgl. Müller \& TeschRömer 2017: 162-163). In anderen Fällen hatten die Engagierten ein Lebensalter erreicht, das als Altersgrenze für das spezifische Engagement gilt, bei der Freiwilligen Feuerwehr liegt diese Altersgrenze z. B. in der Regel bei 65 Jahren (zu Altersgrenzen im Ehrenamt vgl. Künemund \& Vogel 2018). Das Erreichen einer Altersgrenze als Beendigungsgrund trifft auf 38 Prozent der 65-Jährigen und Älteren zu (hierbei waren Mehrfachnennungen erlaubt, das heißt es konnten mehrere Gründe für die Beendigung angegeben werden, vgl. Müller \& Tesch-Römer 2017: 162-63). Die Gründe, die dazu beitragen, dass Menschen im Lebenslauf ein ehrenamtliches Engagement beenden, können zum anderen auch in anderen Lebensbereichen liegen, z. B. werden von jeweils rund einem Drittel der 65-Jährigen und Älteren berufliche oder familiäre Gründe dafür genannt, dass sie ein früheres Engagement beenden mussten, und von rund ei- 
nem Viertel gesundheitliche Gründe. Darüber hinaus hat rund ein Drittel der 65-Jährigen und Älteren ein früheres Engagement beendet, weil sie keine Verpflichtungen mehr haben wollten (zu den Beendigungsgründen vgl. Müller \& Tesch-Römer 2017: 162-163).

\section{Alternsverläufe ehrenamtlichen Engagements von Frauen und Männern}

Wie verändern sich die Wahrscheinlichkeiten, sich ehrenamtlich zu engagieren, bei Frauen und Männern mit dem Älterwerden? Und unterscheiden sich die Alternsverläufe ehrenamtlichen Engagements von Frauen und Männern? Aus Querschnittsanalysen auf Basis des Deutschen Alterssurveys ist bekannt, dass Frauen im Alter von 40 bis 85 Jahren zu etwas geringeren Anteilen ehrenamtlich engagiert sind (19 Prozent im Jahr 2014) als Männer (26 Prozent im Jahr 2014) (vgl. Wetzel \& Simonson 2017: 87; für 1996 und 2002 vgl. Künemund 2006; für 2008 vgl. Naumann \& Romeu Gordo 2010). Allerdings ist der Geschlechterunterschied in der Engagementbeteiligung in verschiedenen Altersgruppen unterschiedlich ausgeprägt: Während die Engagementquote bei Frauen über 64 Jahren deutlich unter der Engagementquote der Männer dieser Altersgruppe liegt, sind Frauen im Alter zwischen 30 und 49 Jahren zu einem etwas höheren Anteil freiwillig engagiert als Männer (vgl. Vogel et al. 2017: 105).

Diese Befunde deuten darauf hin, dass das Engagement in bestimmten Lebensphasen biografisch mit spezifischen Lebenssituationen verknüpft ist, etwa dem Leben mit minderjährigen Kindern im Haushalt. In einer Lebenslaufbetrachtung könnte es sein, dass mit einer Veränderung der Lebenssituation eine Veränderung der ehrenamtlichen Partizipation einhergeht, beispielsweise wenn beim Verlassen der Kinder des elterlichen Haushaltes Gelegenheiten, sich als Elternvertretung oder im Sportverein der Kinder zu engagieren, wegfallen. Vor allem für Mütter, die vergleichsweise häufig im Kontext von Kindergarten und Schu- le ehrenamtlich tätig sind, könnten die Möglichkeiten, sich ehrenamtlich zu engagieren, somit mit steigendem Alter weniger werden. Umgekehrt könnte aber auch besonders in der Familienphase eine höhere Belastung von Frauen zu erwarten sein, welche die ehrenamtliche Partizipation beschränken könnte - ähnlich wie die Erwerbspartizipation. Da Frauen noch immer häufiger und im größeren zeitlichen Umfang Sorgetätigkeiten in der Familie übernehmen, sind sie somit durch die Übernahme von familiären Verpflichtungen stärker eingeschränkt als Männer (vgl. Kapitel 6 Unbezahlte Sorgetätigkeiten in diesem Band). Bei Männern könnten sich hingegen zusätzlich Gelegenheiten zum Engagement über andere Lebensbereiche wie der beruflichen Tätigkeiten in allen biografischen Phasen bis in den Ruhestand hinein ergeben (zur Bedeutung der früheren beruflichen Tätigkeit für das Engagement im Alter vgl. Maurer 2018).

Untersucht wird, ob sich das ehrenamtliche Engagement im Alternsverlauf von Frauen und Männern unterscheidet. Erwerbstätigkeit und familiäre Verpflichtungen beeinflussen das ehrenamtliche Engagement, so dass die ehrenamtliche Partizipation sich mit dem Alter und den verschiedenen Lebensphasen entwickelt. Da die Familienphase, etwa Kindererziehungszeiten, aber auch Sorgetätigkeiten wie Pflege, die Erwerbspartizipation der Frauen in jüngerem Alter anders als die der Männer beeinflusst, ist zu erwarten, dass das ehrenamtliche Engagement der Frauen auch in der Familienphase anders ist als das der Männer. Oder allgemeiner ausgedrückt, dass das Engagement von Frauen und Männern sich im Verlauf zwischen 40 und 90 Jahren unterscheidet. Mit den im nachfolgenden präsentierten Längsschnittanalysen lässt sich erstmals belegen, wie stark sich die Wahrscheinlichkeit, sich ehrenamtlich zu engagieren, für Frauen und Männer mit dem Älterwerden verändert. 


\section{Sozialer Wandel der Alternsverläufe ehrenamtlichen Engagements}

Das ehrenamtliche Engagement hat sich in den letzten 20 Jahren stark ausgeweitet, der Anteil Engagierter hat sich nahezu verdoppelt: 1996 waren rund 12 Prozent der 40- bis 85-Jährigen ehrenamtlich in Vereinen oder Organisationen engagiert, 2014 waren es bereits gut 22 Prozent (Wetzel \& Simonson 2017). Diesen Anstieg gab es sowohl bei den Frauen (von 8 Prozent auf 19 Prozent) als auch bei den Männern (von 17 Prozent auf 26 Prozent). Dieser Trend ist auch durch Befunde anderer Studien wie dem Deutschen Freiwilligensurvey zu belegen, in dem auch freiwilliges Engagement berücksichtigt wird, welches nicht organisationsgebunden ist (vgl. Vogel et al. 2017). Die auf Basis des Deutschen Alterssurveys ausgewiesenen Quoten ehrenamtlichen Engagements beschreiben organisationsgebundenes ehrenamtliches Engagement. Der Anstieg des Engagements ist laut Deutschem Alterssurvey besonders bei jenen über 65 Jahren überdurchschnittlich ausgeprägt (vgl. Wetzel \& Simonson 2017: 83). Es kann daher vermutet werden, dass sich die Möglichkeiten, sich ehrenamtlich zu engagieren, für später geborene $\mathrm{Ge}-$ burtsjahrgänge verbessert haben. Eine Analyse auf Basis des Sozio-oekonomischen Panels (SOEP) hat ergeben, dass der Anstieg des Engagements zum Teil auf einen Kohortenwandel zurückzuführen ist, denn die vor 1935 Geborenen haben sich seltener ehrenamtlich engagiert als die 1935 bis 1945 geborene „Adenauer-Generation“ (Şaka 2018: 288). Die später geborenen Kohorten der „APO-Generation“ (1946 bis 1953 geboren) und der „Generation der Neuen Sozialen Bewegungen“ (1954 bis 1964 geboren) zeigen jedoch nach Kontrolle von Einflussgrößen wie Bildung keine zusätzliche höhere Engagementbeteiligung (Şaka 2018: 289).

Der Anstieg lässt sich vermutlich unter anderem darauf zurückführen, dass die verstärkte Engagementförderung durch die Politik Früchte trägt. Hierzu zählen neben der Förderung von Anlaufstellen für Engagementinteressierte auch die Verbesserung der Anerkennungskultur durch Prei- se und Auszeichnungen oder Ähnliches. Auch die Rahmenbedingungen für Engagement, wie die Klärung des Versicherungsschutzes, haben sich verbessert (vgl. Wetzel \& Simonson 2017: 83). Neben diesen Verbesserungen auf politischer und gesellschaftlicher Ebene gibt es Gründe für den Engagementanstieg auf der individuellen und auf der organisationalen Ebene: Auf der individuellen Ebene ist zu nennen, dass Personen, die heute älter sind, besser ausgebildet sind als dies die Älteren von früher waren. Da Bildung eine wichtige Ressource für ehrenamtliches Engagement ist, lässt sich der Anstieg des Engagements bei den älteren Menschen zum Teil auf die Ausweitung der Qualifikationen zurückführen. Insbesondere sind Frauen heute zu deutlich höheren Anteilen hochgebildet als in der Vergangenheit, weshalb sich eine stärkere Zunahme der Ehrenamtsbeteiligung bei älteren Frauen erwarten lässt (vgl. Vogel et al. 2017: 118; Şaka 2018). Gleichzeitig ist die Erwerbsbeteiligung in den letzten Jahren deutlich angestiegen - vor allem bei Frauen - und das Ruhestandeintrittsalter verschiebt sich weiter nach hinten. Da Erwerbsarbeit Gelegenheiten zum Engagement mit sich bringt, kann sich auch dieser Trend als engagementförderlich erweisen (vgl. Wetzel \& Simonson 2017: 83). Darüber hinaus trägt auch eine bessere Gesundheit dazu bei, dass Menschen sich vermehrt bis ins hohe Alter ehrenamtlich engagieren können. Auf der individuellen Ebene ist die Situation der Familien zu nennen: Es zeigen sich Veränderungen in den Familienstrukturen, zum Beispiel gibt es zunehmend mehr Personen ohne eigene Kinder. Möglicherweise werden in Zukunft wegfallende familiale Hilfestrukturen mehr durch ehrenamtliche Hilfestrukturen ersetzt (vgl. Vogel et al. 2017: 118). Auf der organisationalen Ebene sind als weitere Gründe für den Anstieg des Anteils ehrenamtlich engagierter älterer Menschen zu nennen, dass die Zahl der Vereine in Deutschland stetig steigt und damit die Nachfrage nach Personen, die ehrenamtlich Vereinsfunktionen wie Kassenwart und Vorsitz etc. übernehmen (vgl. Vogel et al. 2017: 118). Insbesondere sind hier auch Vereinsgründungen von Bürgerhilfevereinen $\mathrm{zu}$ 
nennen, deren Zweck häufig in der Hilfe für ältere Menschen und der Unterstützung bei einem selbstständigen Leben bis ins hohe Alter besteht (vgl. z. B. Alisch, Ritter, Glaser, \& Rubin 2018).

Es ist eine offene Frage, ob sich die Geschlechterunterschiede beim ehrenamtlichen Engagement im Alternsverlauf bei später geborenen Kohorten im Vergleich zu früher geborenen Kohorten weniger ausgeprägt darstellen. Eine Reduzierung der Unterschiede im ehrenamtlichen Engagement von älteren Frauen und Männern könnte beispielsweise erwartet werden, weil Frauen stärker von der Bildungsexpansion profitieren konnten als Männer und Frauen der jüngeren Kohorten eine höhere Erwerbspartizipation ausüben als Frauen der älteren Kohorten. Es liegen Studien vor, die zeigen, dass es eine positive Beziehung zwischen Erwerbstätigkeit und außerberuflicher Partizipation gibt (Künemund \& Schupp 2008; Naumann \& Romeu Gordo 2010; Simonson \& Hameister 2017). Wenn Personen nicht mehr erwerbstätig sind, beenden sie auch eher ihr Ehrenamt. Neben einer Verringerung der Geschlechterunterschiede im Alternsverlauf ist aber auch eine Verstärkung zu erwarten, weil die intensivere Erwerbstätigkeits- und Familienphase zwischen 40 und 60 einen Verdrängungseffekt auf die außerberufliche gesellschaftliche Partizipation insbesondere von Frauen später geborener Kohor- ten haben kann. Nach dem Erwerbsaustritt würde dieser Verdrängungseffekt wegfallen.

\section{Fragestellungen}

Wir analysieren in diesem Kapitel die kohortenspezifischen Alternsverläufe von Frauen und von Männern mit Blick auf ihr ehrenamtliches Engagement. Dabei erlaubt die Datenstruktur des Deutschen Alterssurveys, die früher geborene Kohorte der 1930 bis 1939 Geborenen mit den später geborenen Kohorten der Geburtsjahrgänge 1940 bis 1949 und 1950 bis 1959 zu vergleichen. Während die in den 1930er Jahren Geborenen heute bereits ein hohes Alter erreicht haben, hat ein Großteil der in den 1950er Jahren Geborenen heute bereits den Übergang in den Ruhestand erfahren oder steht kurz vor dem Übergang in den Ruhestand. Im Vordergrund stehen die Fragen, wie sich das ehrenamtliche Engagement später geborener Kohorten über die zweite Lebenshälfte hinweg unterscheidet vom ehrenamtlichen Engagement früher geborener Kohorten, und ob sich die Geschlechterunterschiede im ehrenamtlichen Engagement in den Alternsverläufen später geborener Kohorten verringern.

\section{Indikatoren}

Organisationsgebundenes ehrenamtliches Engagement wird im Deutschen Alterssurvey bei Personen erfasst, die Mitglied in einer Gruppe oder einer Organisation sind, z.B. in einem Sportverein, in einer religiösen Gruppe oder in der Freiwilligen Feuerwehr. Als ehrenamtlich engagiert geht in die Analysen ein, wer angibt, Mitglied in einer Gruppe oder Organisation zu sein und im Anschluss die Frage bejaht „Üben Sie dort eine Funktion aus oder haben Sie ein Ehrenamt inne?“. Da 
viele Menschen in mehreren Vereinen oder Organisationen tätig sind, werden maximal fünf Mitgliedschaften pro Person erfasst. Die im Interview verwendete Liste der Gruppen und Organisationen umfasst die Kategorien: Selbsthilfegruppen, Bürgerinitiativen, Wohltätige Organisationen, die Freiwillige Feuerwehr, Hobby- und Sammelvereine, Gesellige Vereinigungen, Sportvereine, Flüchtlings- oder Vertriebenenverbände, Gewerkschaften, Unternehmens- und Berufsverbände, Politische Parteien, Kirchliche oder religiöse Gruppen, Heimatvereine oder Bürgervereine, Kulturvereine, Musik- und Tanzgruppen sowie Andere.

Personen ab 50 Jahren werden zusätzlich gefragt, ob sie in einer Gruppe mitmachen, die sich besonders „an ältere Menschen im Ruhestand oder im Übergang in den Ruhestand richten“. Die im Interview verwendete Liste umfasst diese Kategorien: Vorruhestandsgruppen oder Gruppen zur Vorbereitung auf den Ruhestand, Seniorenselbsthilfegruppen oder Seniorengenossenschaften, Gruppen für freiwillige Tätigkeiten und Hilfen, Seniorenarbeit der Parteien oder Gewerkschaften sowie Seniorenvertretung und -beiräte, Seniorenuniversitäten und -akademien oder Weiterbildungsgruppen, betriebliche Seniorengruppen, Seniorenfreizeitstätten oder Seniorentreffpunkte, andere Seniorengruppen. Als ehrenamtlich engagiert geht in die Analysen ein, wer bezüglich einer solchen Gruppe die Frage bejaht „Üben Sie dort eine Funktion aus oder haben Sie ein Ehrenamt inne?". Auch hier können Personen wieder bis zu fünf Gruppen nennen.

Um Aussagen über ehrenamtliches Engagement insgesamt treffen zu können, wird zusammen betrachtet, ob sich Menschen allgemein in Organisationen oder aber in Seniorenorganisationen engagieren. Da ehrenamtliches Engagement darüber hinaus auch außerhalb von Mitgliederorganisationen wie Vereinen oder Verbänden ausgeübt werden kann, etwa im kommunalen Bereich als Schöf$\mathrm{fe}$, als ehrenamtlicher Bürgermeister oder in der informellen Nachbarschaftshilfe, ist für die Interpretation der Ergebnisse dieses Kapitels von Bedeutung, dass es sich allein um organisationsge- bundenes ehrenamtliches Engagement und somit nur um einen Ausschnitt des von Menschen in der zweiten Lebenshälfte ausgeübten ehrenamtlichen Engagements insgesamt handelt. Die Anteile ehrenamtlich Engagierter ist im Deutschen Alterssurvey möglicherweise minimal überschätzt, da Menschen, die sich ehrenamtlich engagieren, auch häufiger bereit sind an wissenschaftlichen Studien teilzunehmen, als Menschen, die sich nicht engagieren (vgl. u. a. Abraham, Helms und Presser 2009). Dennoch lassen sich hier gültige Aussagen über die Wahrscheinlichkeit ehrenamtlichen Engagements im Alternsverlauf im Sinne von mit dem Alter steigenden oder sinkenden Wahrscheinlichkeiten treffen.

In der Darstellung der Ergebnisse unterscheiden wir zwischen der mittleren Wahrscheinlichkeit, ein Ehrenamt auszuüben, die im Folgenden als "Ausübung eines Ehrenamtes" beschrieben wird, der mittleren Wahrscheinlichkeit, ein Ehrenamt aufzunehmen und der mittleren Wahrscheinlichkeit, ein Ehrenamt zu beenden, die als „Neuaufnahme eines Ehrenamtes“ und als „Beendigung eines Ehrenamtes" beschrieben werden. Personen erhalten den Wert 1 für „Neuaufnahme“, wenn sie in der betrachten Erhebungswelle ein Ehrenamt ausüben, in der vorherigen Erhebungswelle aber kein Ehrenamt ausgeübt haben. Den Wert 0 "Keine Neuaufnahme“ erhalten alle Personen, die in der betrachteten Erhebungswelle kein Ehrenamt ausüben sowie Personen, die in der betrachteten Erhebungswelle ein Ehrenamt ausüben, aber auch in der vorherigen Erhebungswelle bereits ein Ehrenamt ausgeübt haben. Das Vorgehen für die Beendigung ist äquivalent (Wert 1 „Beendigung“ erhalten Personen, die in der betrachteten Erhebungswelle kein Ehrenamt ausüben, in der vorherigen Erhebungswelle aber ein Ehrenamt ausgeübt haben).

\section{Statistisches Analyseverfahren}

Untersucht wird, wie hoch die durchschnittliche Wahrscheinlichkeit in der Population über die Al- 
tersspanne von 40 bis 90 Jahren ist, zu einem bestimmten Alter im Alternsverlauf eine ehrenamtliche Tätigkeit auszuüben. Zudem wird untersucht, wie hoch die durchschnittliche Wahrscheinlichkeit im Alter von 40 bis 90 Jahren ist, zu einem bestimmten Alter eine neue ehrenamtliche Tätigkeit aufzunehmen beziehungsweise im Alternsverlauf eine ehrenamtliche Tätigkeit zu beenden. In der Ergebnisdarstellung werden die in Analysemodellen geschätzten Wahrscheinlichkeiten als Prozentanteile ausgegeben. Auf Basis des Designs des Deutschen Alterssurveys ist es möglich, den gesamten Verlauf über 50 Jahre (also von 40 bis 90 Jahre) zu schätzen. Für jeden Geburtsjahrgang liegen Angaben über einen Zeitraum von maximal 21 Jahren zugrunde (zum Stichprobendesign siehe Kapitel 2 Design, Inhalte und Methodik des Deutschen Alterssurveys in diesem Band).

Die Modellschätzungen erfolgten mit Hilfe logistischer Panelregressionen. Es wurden eine li- neare sowie eine quadratische Alterskomponente aufgenommen, um die linearen und kurvilinearen Alternsveränderungen der Wahrscheinlichkeiten, sich zu engagieren, ein Ehrenamt neu aufzunehmen oder ein Ehrenamt zu beenden, abzubilden. Außerdem wurde für Geschlecht und Region kontrolliert, um der geschichteten Stichprobenziehung der DEAS-Basiserhebungen Rechnung zu tragen. Die Unterschiede zwischen den Geburtskohorten wurden anhand der Annahme, dass sich Kohorteneffekte für jede später geborene Geburtskohorte in gleicher Form darstellen werden, als lineare Kohorteneffekte geschätzt (vgl. hierzu ausführlich das Kapitel 2 Design, Inhalte und Methodik des Deutschen Alterssurveys in diesem Band). Die Alternsveränderungen und die Unterschiede nach Geschlecht oder Kohorte wurden in den Modellschätzungen auf statistische Signifikanz getestet, die Irrtumswahrscheinlichkeit wurde bei allen Tests auf $\mathrm{p}<0,05$ festgesetzt.

\subsection{Ergebnisse}

\subsubsection{Ausübung eines Ehrenamtes im Verlauf der zweiten Lebenshälfte}

In Abbildung 7-1 ist der durchschnittliche Alternsverlauf der Ausübung ehrenamtlicher Tätigkeiten separat für Frauen und Männer sowie für die gesamte Untersuchungsstichprobe dargestellt. Allgemein ist die ehrenamtliche Partizipation in Vereinen und Organisationen (nach der Definition von Abschnitt 2) über den hier betrachteten Lebensverlauf von 40 bis 90 Jahren niedrig. In dem analysierten Altersbereich von 40 bis 90 Jahren liegen die Werte unter 20 Prozent für Frauen und knapp über 20 Prozent für Männer. Am höchsten ist die Wahrscheinlichkeit, sich ehrenamtlich $\mathrm{zu}$ engagieren, im Alter zwischen 53 und 63 Jahren.

Über die zweite Lebenshälfte steigt die Wahrscheinlichkeit, sich ehrenamtlich zu engagieren, zunächst an, später sinkt sie mit steigendem Lebensalter jedoch wieder $a b$.

Vom Alter von 40 bis zum Alter von 58 Jahren steigt die Wahrscheinlichkeit stetig an, sich ehrenamtlich zu engagieren (Abbildung 7-1). Ab einem Alter von 59 Jahren nimmt die Wahrscheinlichkeit, ehrenamtlich engagiert $\mathrm{zu}$ sein, mit steigendem Alter stetig ab (Abbildung 7-1).

Über die gesamte zweite Lebenshälfte hinweg haben Männer eine höhere Wahrscheinlichkeit, sich ehrenamtlich zu engagieren, als Frauen.

Bei Männern steigt die durchschnittliche Wahrscheinlichkeit, ehrenamtlich engagiert zu sein, von 17 Prozent (im Alter von 40 Jahren) auf ein Maximum von 23 Prozent (mit 57 Jahren) und sinkt später auf sieben Prozent (mit 90 Jahren). Bei Frauen steigt diese Wahrscheinlichkeit von elf Prozent auf 
Abbildung 7-1 Ausübung eines Ehrenamtes bei Frauen und Männern im Alternsverlauf

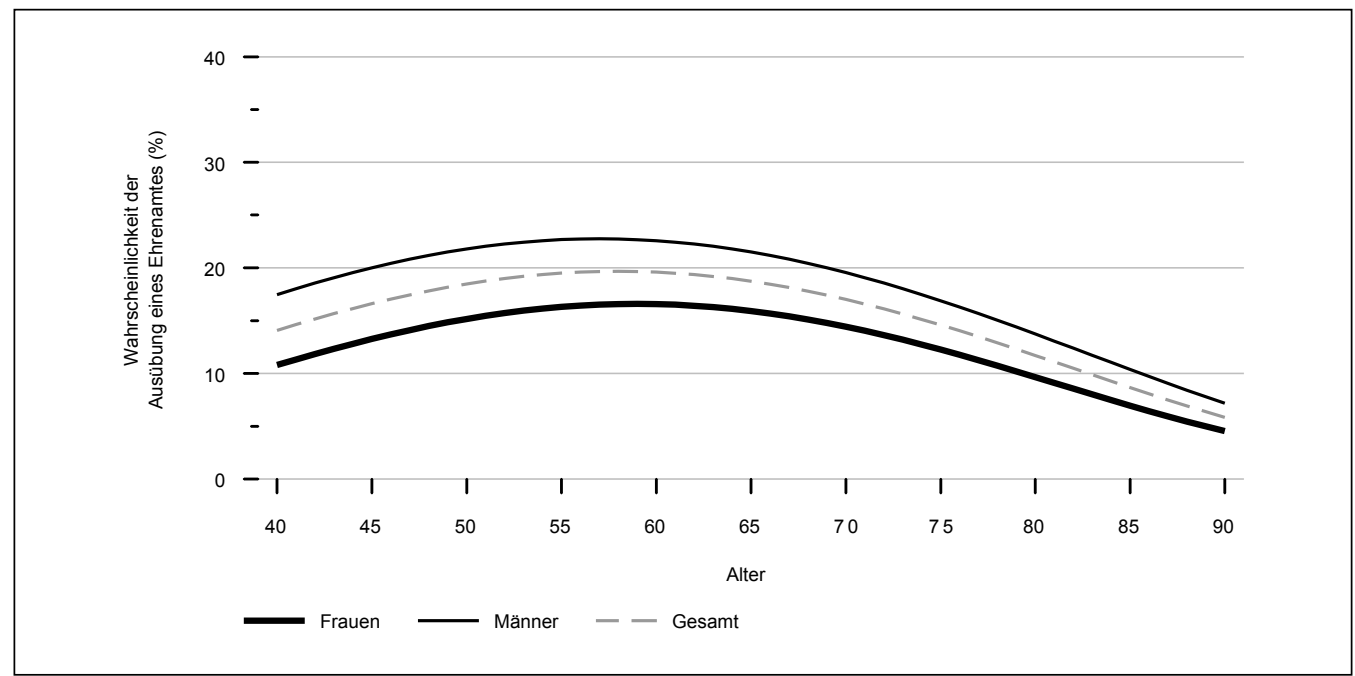

Quelle: DEAS 1996-2017, Schätzungen basieren auf n = 20070 Studienteilnehmenden (10 174 Männer und 9876 Frauen) mit insgesamt $\mathrm{n}=39280$ Beobachtungen; kontrolliert für Region.

Bei Frauen steigt die Wahrscheinlichkeit, ehrenamtlich engagiert zu sein, von elf Prozent (mit 40 Jahren) auf ein Maximum von 17 Prozent (mit 6o Jahren) und sinkt auf fünf Prozent (mit 90 Jahren). Bei Männern steigt die Wahrscheinlichkeit, ehrenamtlich engagiert zu sein, auf ein Maximum von 23 Prozent (mit 6o Jahren) und sinkt später auf sieben Prozent ab (mit 90 Jahren).

ein Maximum von 17 Prozent (mit 59 Jahren) und sinkt mit steigendem Lebensalter auf fünf Prozent (mit 90 Jahren). Über den gesamten Alternsverlauf ist die Wahrscheinlichkeit der Ausübung eines Ehrenamtes somit höher für Männer als für Frauen (Abbildung 7-1). Allerdings sind die Geschlechterunterschiede der Wahrscheinlichkeit, ein Ehrenamt auszuüben, in der Erwerbsphase größer als in der Ruhestandsphase (ab einem Alter von 88 Jahren ist der Unterschied zwischen Frauen und Männern nicht mehr statistisch signifikant).

Die Wahrscheinlichkeit, ehrenamtlich engagiert zu sein, ist für später geborene Kohorten über den gesamten betrachteten Alternsverlauf höher als für früher geborene Kohorten.

In Abbildung 7-2 sind die Alternsverläufe für drei Geburtskohorten dargestellt: für die zwischen 1930 und 1939 Geborenen; für die zwischen 1940 und
1949 Geborenen und für die zwischen 1950 und 1959 Geborenen. Allgemein kann festgehalten werden, dass später geborene Kohorten eine höhere Wahrscheinlichkeit ehrenamtlicher Partizipation im Alternsverlauf zeigen als früher geborene Kohorten (Abbildung 7-2). Die Form der Wahrscheinlichkeitsverläufe scheint für die Kohorten ähnlich, für alle drei Geburtskohorten steigt die Wahrscheinlichkeit, ehrenamtlich tätig zu sein, zunächst mit dem Lebensalter an. Darüber hinaus scheint sich der Wendepunkt, zu dem ein Rückgang des Ehrenamtes mit steigendem Alter eintritt, biografisch nach hinten zu verschieben. Während für die früher geborene Kohorte (1930 bis 1939 geboren) ein Rückgang ab einem Alter von 70 Jahren zu beobachten ist, ist das nicht der Fall für die zwischen 1940 und 1949 geborenen Personen. Für diese mittlere Kohorte beobachten wir die Partizipation bis 77 Jahre, jedoch ist bis zu diesem Alter kein Rückgang zu beobachten, der Wendepunkt 
tritt also voraussichtlich biografisch noch später ein. Auch der Verlauf der jüngsten Kohorte (1950 bis 1959) zeigt in dem zuletzt beobachteten Alter (67 Jahre) immer noch eine steigende Tendenz. Das heißt, die Wahrscheinlichkeit, im höheren Alter engagiert zu sein, ist bei den später Geborenen höher als bei den früher Geborenen, sie steigt mit dem Alter über eine ausgedehntere Altersspanne an und sie nimmt voraussichtlich erst ab einem höheren Alter ab.

Wenn sich die derzeitigen gesellschaftlichen Bedingungen in der Zukunft nicht gravierend verändern, werden sich somit voraussichtlich immer mehr Personen auch in einem höheren Lebensalter ehrenamtlich engagieren. Unsere Analysen zeigen, dass sich der Wendepunkt, zu dem ein Rückgang der Wahrscheinlichkeit, ein Ehrenamt auszuüben, erfolgt, in der zweiten Lebenshälfte weiter nach hinten verschieben wird. Voraussichtlich wird sich die Phase, in der Personen im Alter ehrenamtlich aktiv bleiben, somit verlängern.
Die Geschlechterunterschiede im Alternsverlauf reduzieren sich nicht für später geborene Kohorten.

Sowohl für Frauen als auch für Männer ist die Wahrscheinlichkeit, ehrenamtlich engagiert $\mathrm{zu}$ sein, für später geborene Kohorten über den gesamten beobachteten Alternsverlauf höher als für früher geborene Kohorten. Abbildung 7-3 zeigt für alle drei Kohorten, dass die Wahrscheinlichkeit, ein Ehrenamt auszuüben, für Männer höher ist als für Frauen und das zu jedem Zeitpunkt im Alternsverlauf. Der in Abbildung 7-2 dargestellte soziale Wandel im Ehrenamt unterscheidet sich nicht für Frauen und Männer. Das bedeutet auch, dass die Geschlechterunterschiede im Alternsverlauf in jeder Kohorte relativ konstant bleiben. Dass sich die Ehrenamtsbeteiligungen der Frauen und Männer im Alternsverlauf über die Kohorten ähnlich entwickeln, bedeutet also, dass es keine Annäherung der Ehrenamtswahrscheinlichkeit zwischen den Geschlechtern gibt.

\section{Abbildung 7-2 Ausübung eines Ehrenamtes nach Kohorten im Alternsverlauf}

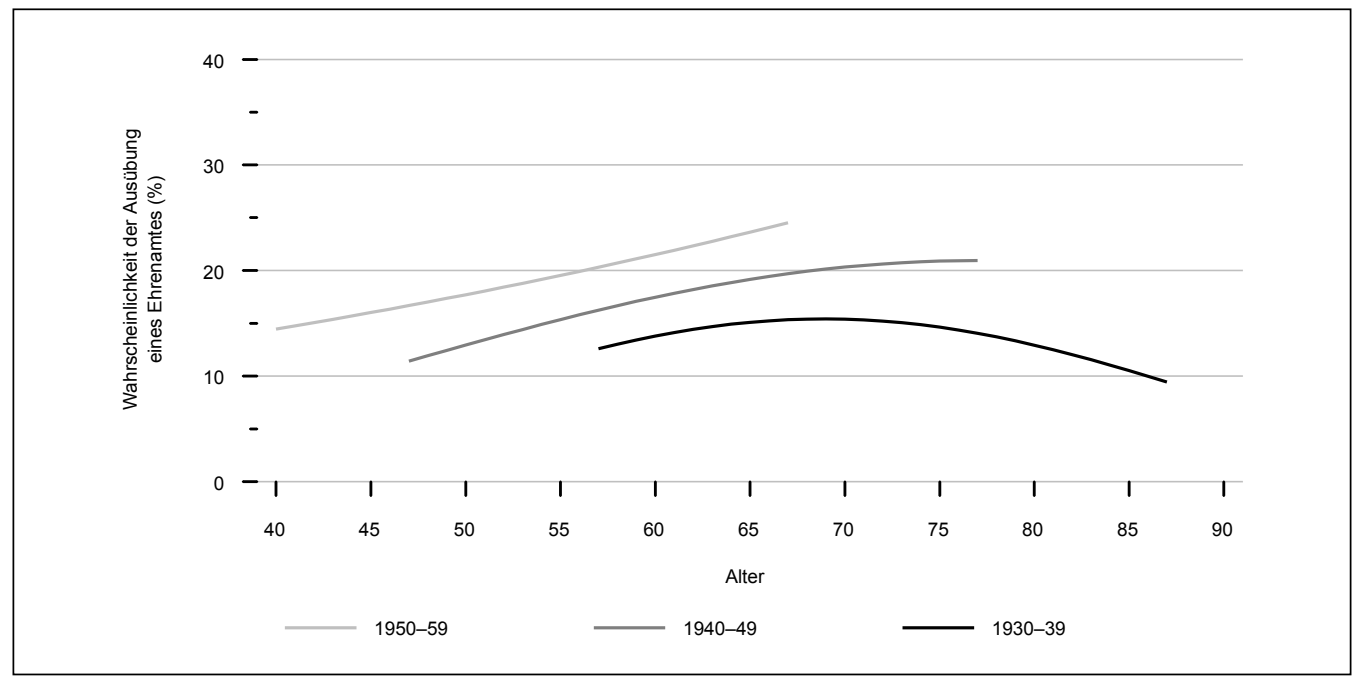

Quelle: DEAS 1996-2017, Schätzungen basieren auf n = 13966 Studienteilnehmenden mit insgesamt n = 29125 Beobachtungen; kontrolliert für Region und Geschlecht.

Für die älteste Kohorte (1930 bis 1939) ist ein Rückgang im ehrenamtlichen Engagement ab einem Alter von 72 Jahren zu beobachten. Für die zwischen 1940 und 1949 geborenen Personen ist bis zum Alter von 77 Jahren (bis zu diesem Alter ist diese Kohorte im DEAS zu beobachten) kein Rückgang zu beobachten. 
Abbildung 7-3 Ausübung eines Ehrenamtes bei Frauen und Männern nach Kohorten im Alternsverlauf

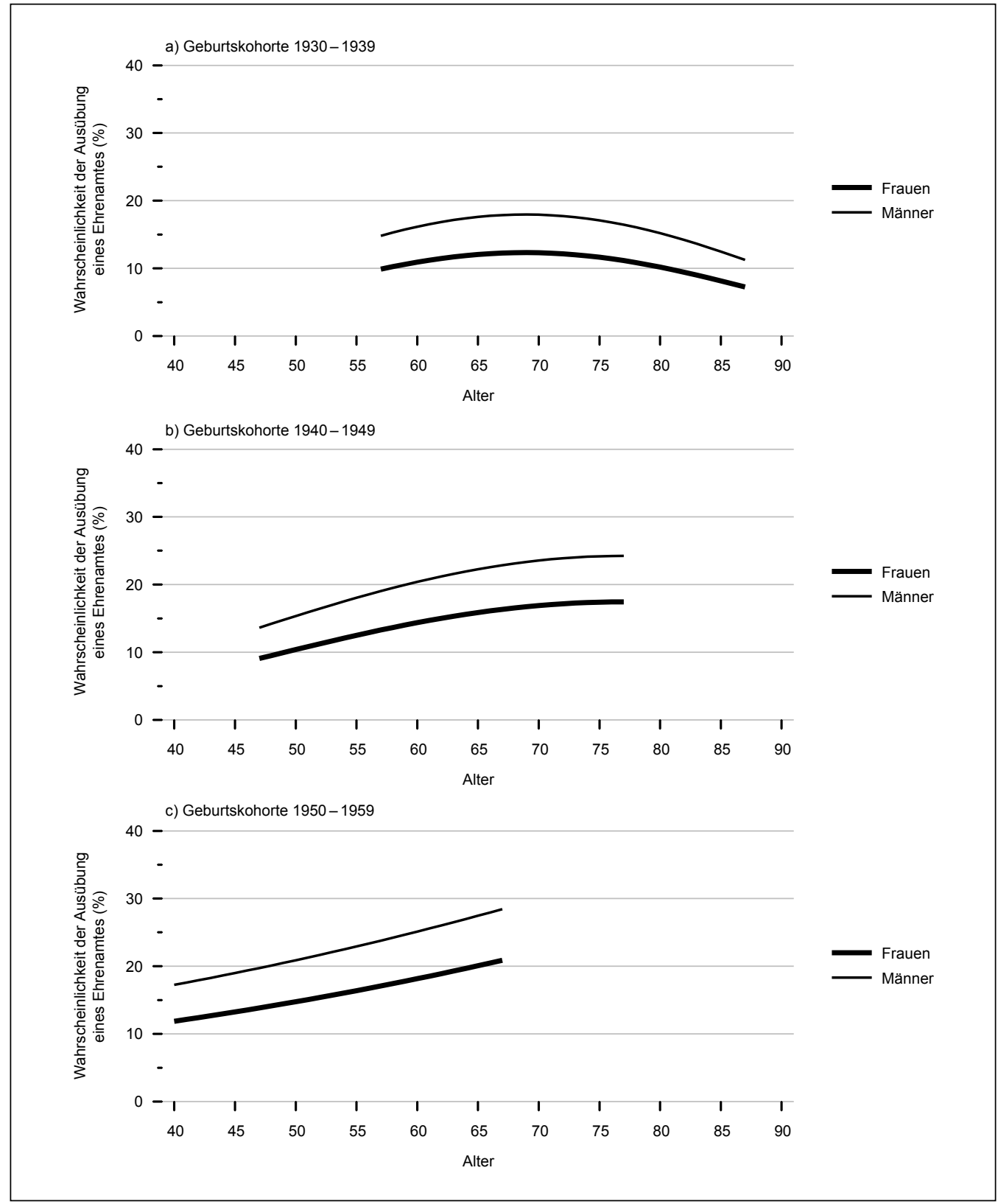

Quelle: DEAS 1996-2017, Schätzungen basieren auf $n=13966$ Studienteilnehmenden mit insgesamt $n=29125$ Beobachtungen; kontrolliert für Region.

Für alle drei Kohorten sind die Alternsverläufe der Wahrscheinlichkeit, ein Ehrenamt auszuüben, für Frauen und Männer ähnlich, so dass es keine Annäherung zwischen den Geschlechtern über die Kohorten hinweg gibt. 


\subsubsection{Neuaufnahme und Beendigung von ehrenamtlichem Engagement im Verlauf der zweiten Lebenshälfte}

Im Durchschnitt steigt die mittlere Wahrscheinlichkeit ehrenamtlicher Partizipation ab 40 Jahren bis ins 6. Lebensjahrzehnt stetig an. Diese Steigerung kommt dadurch zustande, dass in dieser Lebensphase der Anteil von Personen, die ein Ehrenamt neu aufnehmen, höher ist als der Anteil der Personen, die ein solches beenden (Abbildung 7-4).

Die durchschnittliche Wahrscheinlichkeit, ein Ehrenamt auszuüben, ergibt sich zum einen durch die Neuaufnahmen ehrenamtlicher Tätigkeiten und zum anderen durch die Beendigungen ehrenamtlicher Tätigkeiten.

Neuaufnahmen nehmen mit steigendem Lebensalter zunächst bis zu einem Alter von 54 Jahren zu und dann nehmen sie mit steigendem Lebensalter ab. Das heißt, mit höherem Lebensalter werden die Personen, die zuvor nicht ehrenamtlich tätig waren, auch seltener ehrenamtlich aktiv. Es sollte beachtet werden, dass auch wenn nach dem Erreichen der Regelaltersgrenze des Renteneintritts, die derzeit schrittweise von 65 auf 67 Jahre angehoben wird, immer noch Personen ein neues Ehrenamt aufnehmen, die Neuaufnahmen in diesem Alter somit niedriger sind als vor dem Renteneintritt. Die Personen fangen eher mit einem Ehrenamt an, wenn sie im Erwerbsalter sind als wenn sie sich bereits im Ruhestandsalter befinden (Abbildung 7-4). Darüber hinaus ist bis zu einem Alter von 64 Jahren die Wahrscheinlichkeit von Personen, die eine ehrenamtliche Aktivität neu aufnehmen, höher als jene von Personen, die eine ehrenamtliche Aktivität beenden. $\mathrm{Ab}$ einem Alter von 65 Jahren bleibt der Anteil der Beendigungen stabil, während die Neuaufnahmen zurückgehen. Die stetig sinkende Wahrscheinlichkeit, im höheren Alter einer ehrenamtlichen Tätigkeit nachzugehen, ergibt sich so-

Abbildung 7-4 Neuaufnahme und Beendigung eines Ehrenamtes im Alternsverlauf

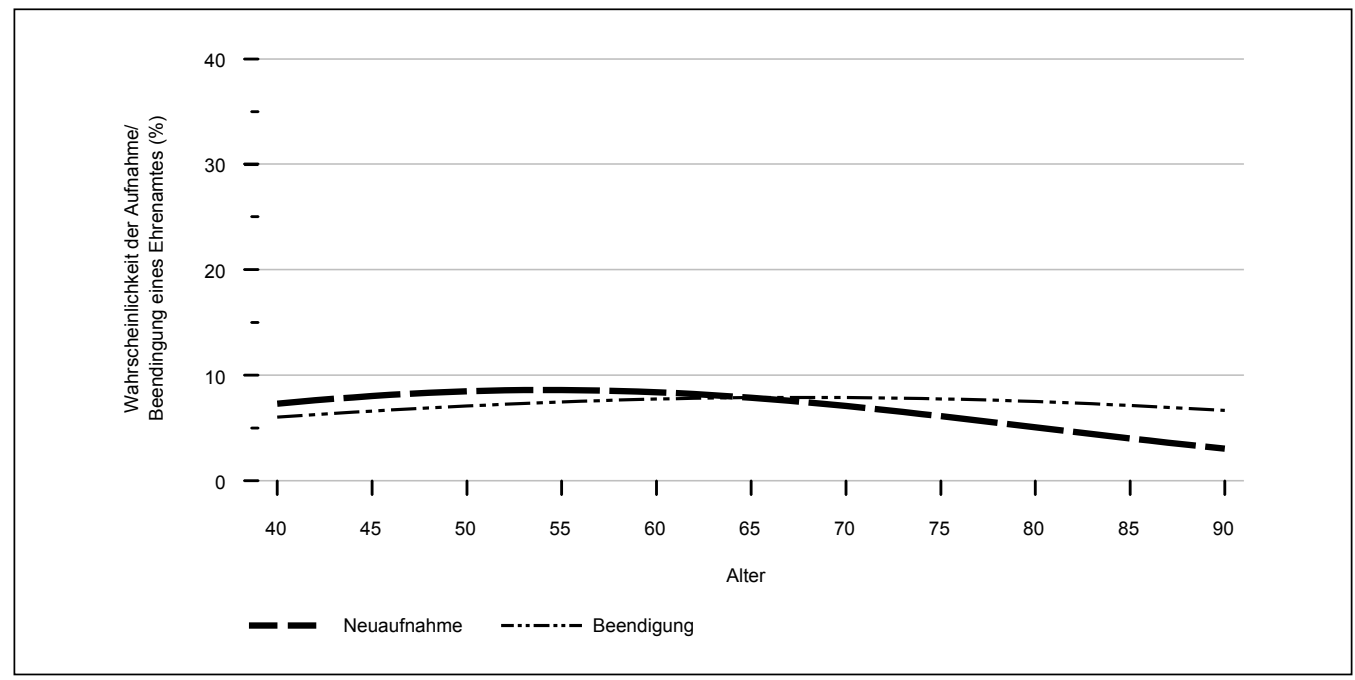

Quelle: DEAS 1996-2017, Schätzungen basieren auf $n=9513$ (Neuaufnahme) und $n=9519$ (Beendigung) Studienteilnehmenden mit insgesamt $\mathrm{n}=18555$ (Neuaufnahme) und $\mathrm{n}=18561$ (Beendigung) Beobachtungen; kontrolliert für Region und Geschlecht.

Die Wahrscheinlichkeit, ein Ehrenamt aufzunehmen, nimmt mit steigendem Lebensalter zunächst bis zu einem Alter von 55 Jahren zu und dann (ab 56 Jahren) mit steigendem Lebensalter ab. Die Wahrscheinlichkeit, ein Ehrenamt zu beenden, verändert sich kaum über den Verlauf der zweiten Lebenshälfte. 
mit daraus, dass in diesem höheren Lebensalter zunehmend weniger Personen ein Ehrenamt neu anfangen und gleichzeitig kaum weniger Engagierte ihre ehrenamtlichen Tätigkeiten beenden. Diese beiden verschiedenen Prozesse (mehr Neuaufnahmen als Beendigungen in der späten Erwerbsphase und weniger Neuaufnahmen als Beendigungen in der Ruhestandsphase) ergeben zusammen das Profil in Abbildung 7-1.

Diese Verläufe bei den Neuaufnahmen und Beendigungen sind sowohl für Frauen als auch für Männer zu beobachten. Bei den Neuaufnahmen sind keine signifikanten Geschlechterunterschiede im Alternsverlauf zu beobachten (Abbildung 7-5). Im Fall der Beendigungsverläufe können nur kleine Unterschiede festgestellt werden (Abbildung 7-6). Während der Verlauf der Frauen stabiler ist und die Wahrscheinlichkeit, ein Ehrenamt zu beenden, nicht die 7-Prozentgrenze übersteigt, liegt bei den Männern der Wendepunkt höher (mit einer Wahrscheinlichkeit von knapp 9 Prozent). Im Alter 40 und im Alter von 90 Jahren sind die Wahrscheinlichkeiten für Frauen und Männer ähnlich.

Die Wahrscheinlichkeit, ein Ehrenamt neu aufzunehmen beziehungsweise zu beenden, ist für später geborene Kohorten über den gesamten betrachteten Alternsverlauf nicht höher beziehungsweise niedriger als für früher geborene Kohorten.

Die Wahrscheinlichkeit, ein Ehrenamt neu aufzunehmen, nimmt im Alter zunächst zu und danach wieder ab, so dass im hohen Alter eine sehr niedrige Wahrscheinlichkeit für eine Neuaufnahme zu beobachten ist. Im Kohortenvergleich beobachten wir ähnliche Verläufe (Abbildung 7-7), wobei bei Frauen und Männern der später geborenen Kohorte (1950-1959) eine höhere Wahrscheinlichkeit im jüngeren Alter zu beobachten ist, die im Alternsverlauf wieder sinkt. Die Unterschiede zwischen den Geburtskohorten sind nicht statistisch signifikant.

Abbildung 7-5 Neuaufnahme eines Ehrenamtes bei Frauen und Männern im Alternsverlauf

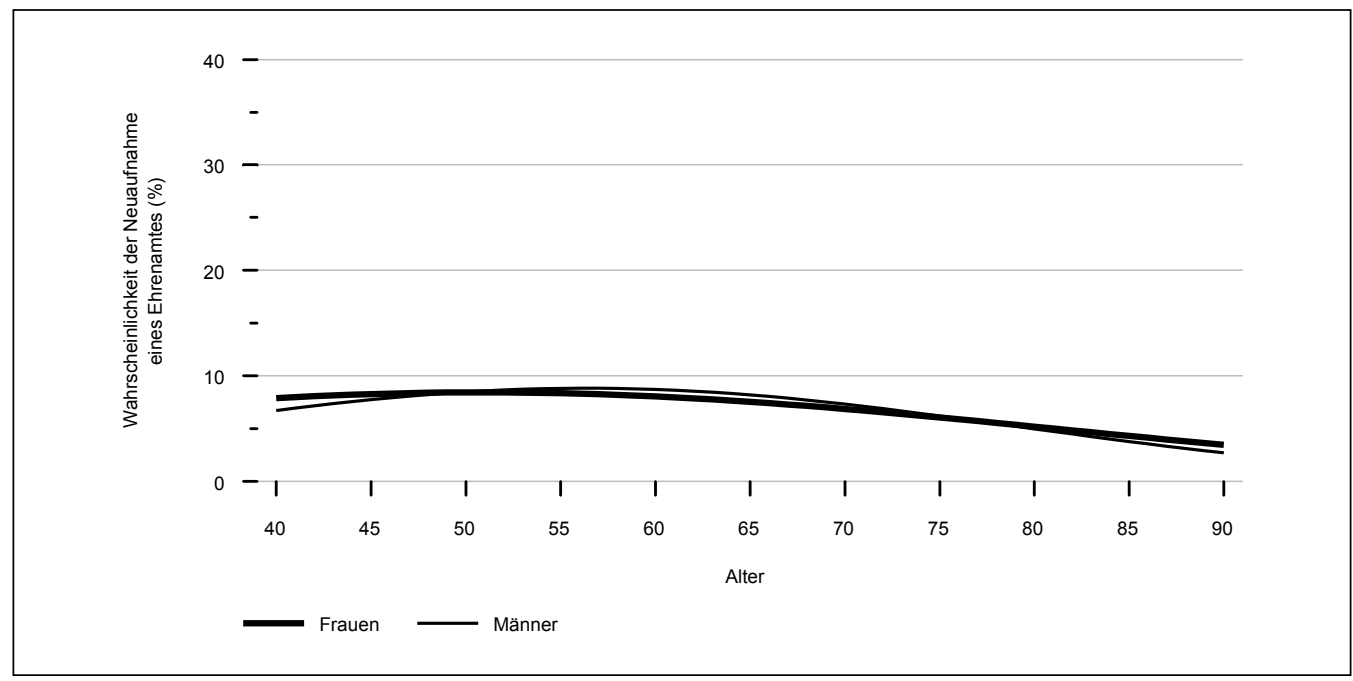

Quelle: DEAS 1996-2017, Schätzungen basieren auf $\mathrm{n}=9513$ Studienteilnehmenden mit insgesamt n = 18555 Beobachtungen; kontrolliert für Region.

Die Wahrscheinlichkeit, ein Ehrenamt neu aufzunehmen, unterscheidet sich nicht signifikant zwischen Frauen und Männern. 


\section{Abbildung 7-6 Beendigung eines Ehrenamtes bei Frauen und Männern im Alternsverlauf}

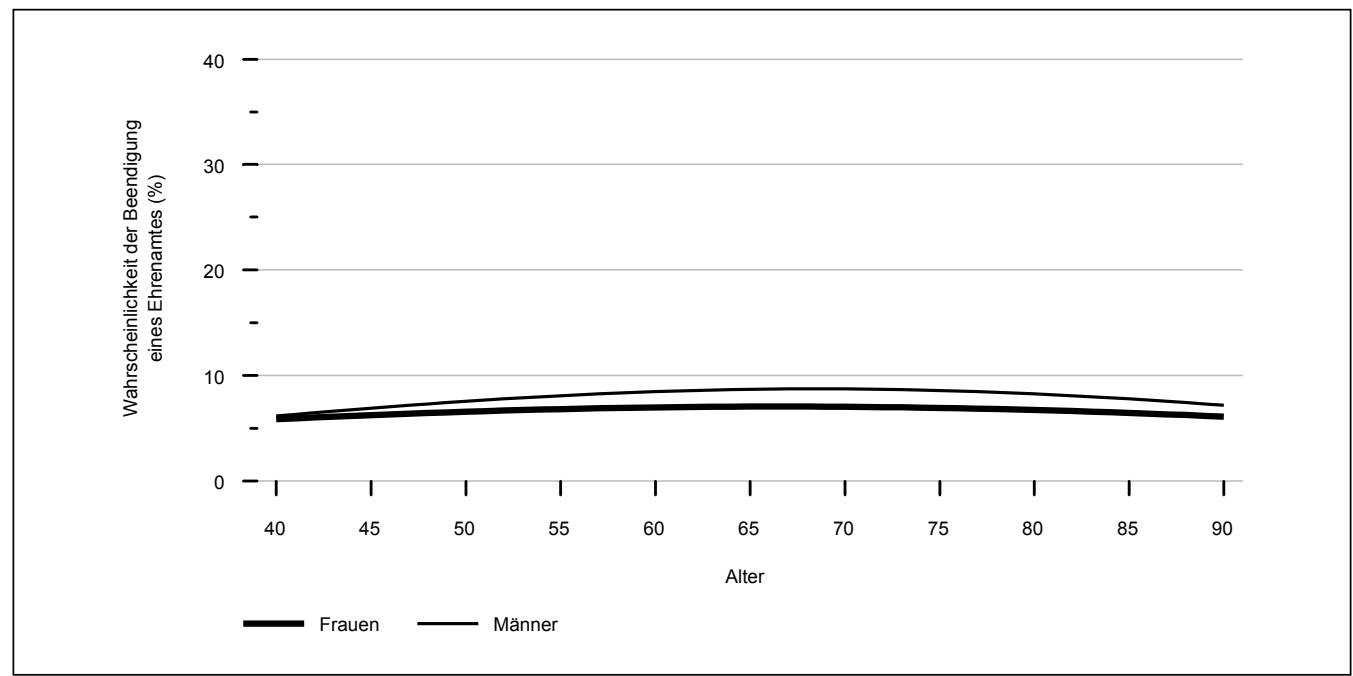

Quelle: DEAS 1996-2017, Schätzungen basieren auf $n=9519$ Studienteilnehmenden mit insgesamt n = 18561 Beobachtungen; kontrolliert für Region.

Die Wahrscheinlichkeit, ein Ehrenamt zu beenden, unterscheidet sich minimal zwischen Frauen und Männern, wobei der Verlauf der Frauen stabiler über den Verlauf der zweiten Lebenshälfte ist.

\section{Abbildung 7-7 Neuaufnahme eines Ehrenamtes nach Kohorten im Alternsverlauf}

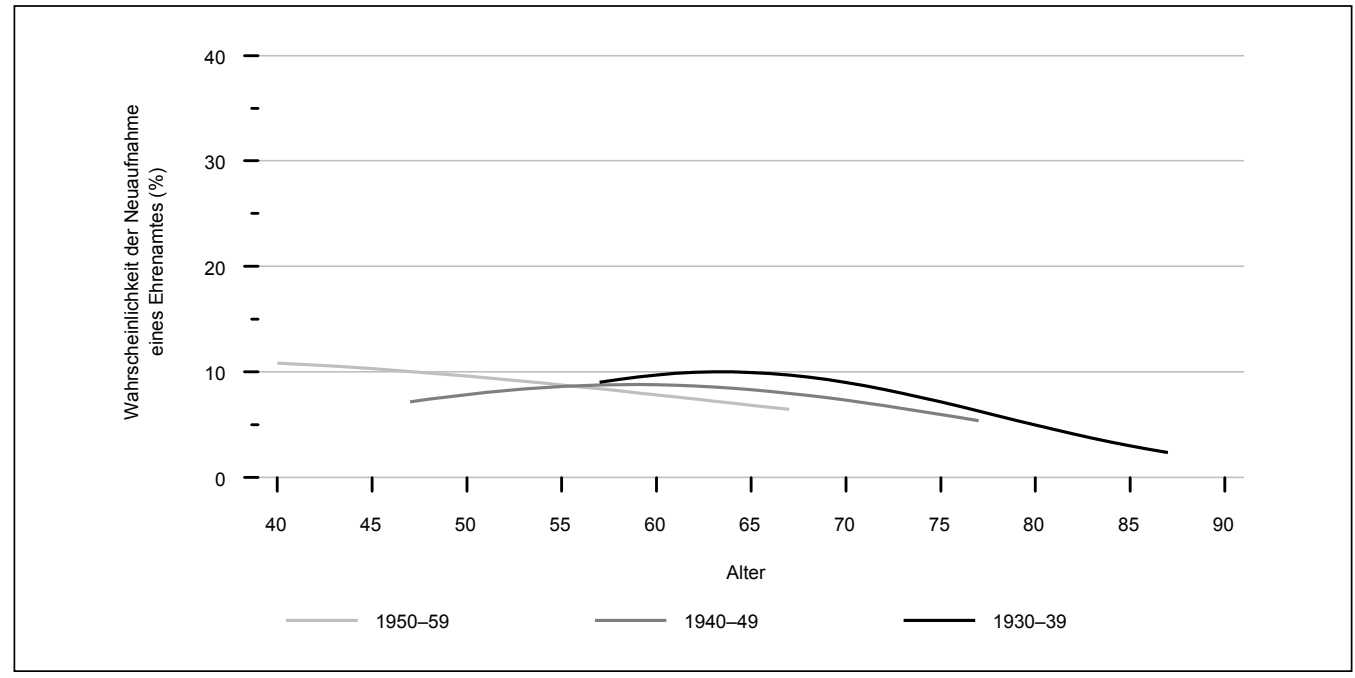

Quelle: DEAS 1996-2017, Schätzungen basieren auf $n=7122$ Studienteilnehmenden mit insgesamt n= 14 625 Beobachtungen; kontrolliert für Region und Geschlecht.

Die Wahrscheinlichkeit, ein Ehrenamt neu anzunehmen, unterscheidet sich im Alternsverlauf nicht signifikant zwischen den Kohorten. 
Abbildung 7-8 Beendigung eines Ehrenamtes nach Kohorten im Alternsverlauf

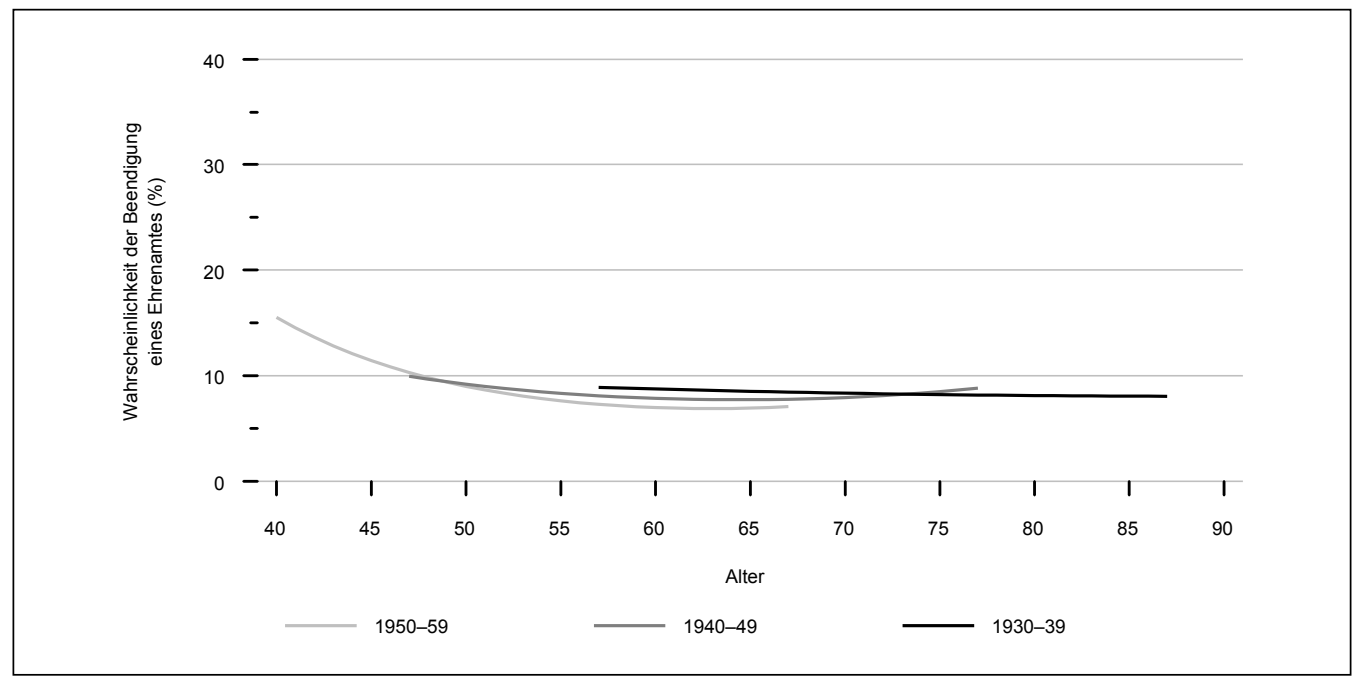

Quelle: DEAS 1996-2017, Schätzungen basieren auf $n=7127$ Studienteilnehmenden mit insgesamt n = 14626 Beobachtungen; kontrolliert für Region und Geschlecht.

Die Wahrscheinlichkeit, ein Ehrenamt zu beenden, unterscheidet sich im Alternsverlauf nicht signifikant zwischen den Kohorten.

Auch bei der Wahrscheinlichkeit, ein Ehrenamt $\mathrm{zu}$ beenden, beobachten wir keinen statistisch signifikanten Unterschied zwischen den Geburtskohorten (Abbildung 7-8). Bei Personen der später geborenen Kohorte (1950-1959) sind höhere Wahrscheinlichkeiten im jüngeren Lebensalter zu beobachten, die dann sinken und sich an die der anderen Geburtskohorten angleichen.

\subsection{Diskussion}

\section{Zusammenfassung}

Sowohl für Frauen als auch für Männer steigt die Wahrscheinlichkeit, sich ehrenamtlich in formalen Organisationen zu engagieren, zunächst mit dem Lebensalter an und sinkt im höheren Alter
Die Geschlechterunterschiede bei der Wahrscheinlichkeit, ein Ehrenamt im Alternsverlauf zu beenden, bleiben für später geborene Kohorten bestehen.

Die geringen Geschlechterunterschiede bei der Wahrscheinlichkeit, ein Ehrenamt zu beenden (Abbildung 7-6), reduzieren sich nicht für später geborene Geburtskohorten. Abbildung 7-9 zeigt, wie diese kleinen Unterschiede zwischen Frauen und Männern relativ konstant bleiben über die Kohorten hinweg.

wieder ab. Der biografische Wendepunkt liegt bei Frauen und Männern bei rund 58 Jahren. Der Geschlechterunterschied besteht hauptsächlich darin, dass Männer über den gesamten betrachteten Verlauf (also von 40 bis 90 Jahren) eine deutlich höhere Wahrscheinlichkeit aufweisen, sich in forma- 
Abbildung 7-9 Beendigung eines Ehrenamtes bei Frauen und Männern nach Kohorten im Alternsverlauf

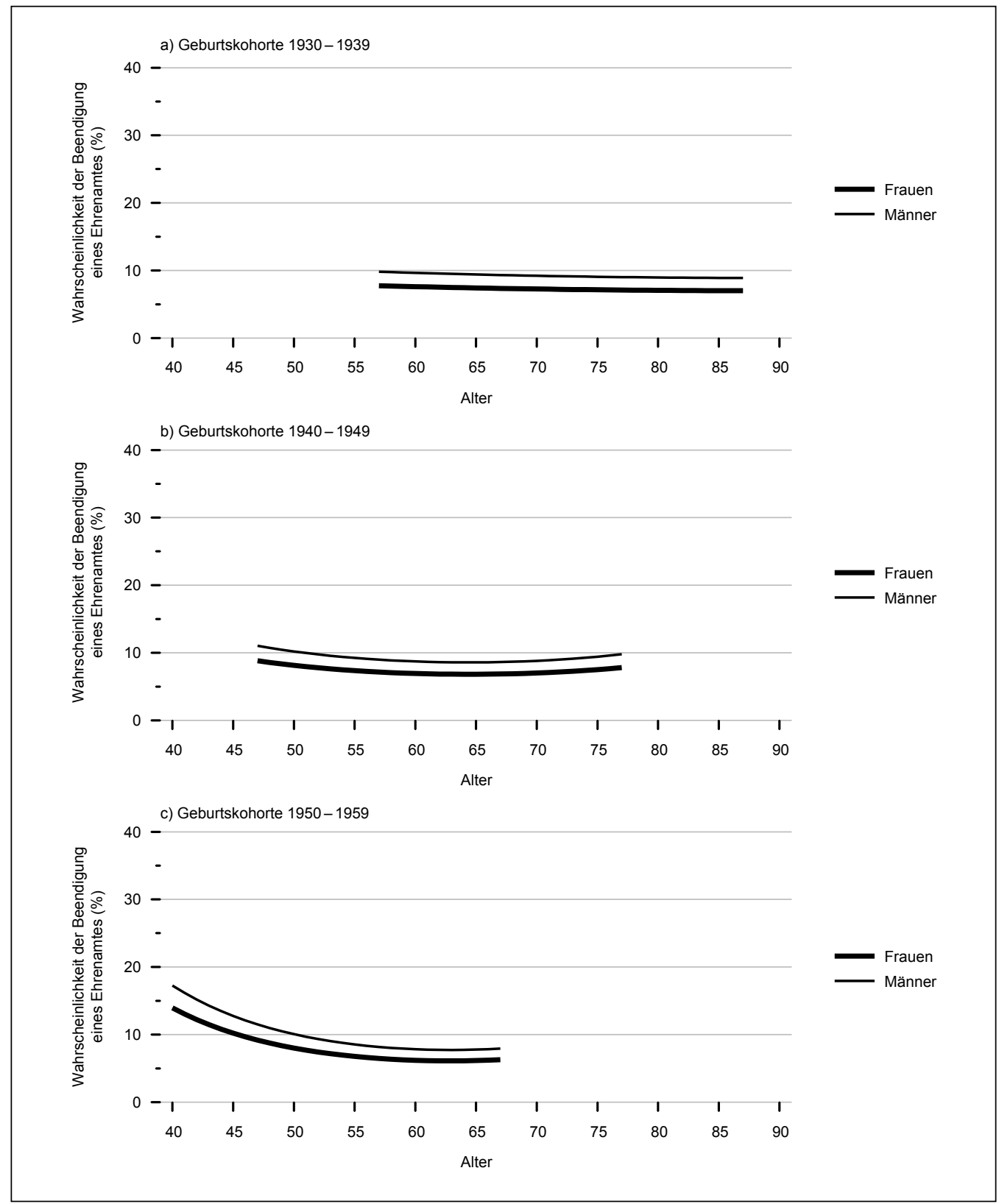

Quelle: DEAS 1996-2017, Schätzungen basieren auf $n=7127$ Studienteilnehmenden mit insgesamt n= 14626 Beobachtungen; kontrolliert für Region.

Die Geschlechterunterschiede in der Wahrscheinlichkeit, im Alternsverlauf ein Ehrenamt zu beenden, bleiben über die Geburtskohorten stabil. 
len Organisationen ehrenamtlich zu engagieren, als Frauen. Dabei ist der Geschlechterunterschied in der Erwerbsphase sogar noch größer als in der Ruhestandsphase, denn der Geschlechterunterschied in der Wahrscheinlichkeit, sich ehrenamtlich zu engagieren, verringert sich mit steigendem Alter insbesondere in der Ruhestandsphase. Später geborene Kohorten haben im Alternsverlauf eine höhere Wahrscheinlichkeit, sich ehrenamtlich zu engagieren, als früher geborene Kohorten. Dieser Befund gilt für Frauen und Männer gleichermaßen. Das bedeutet aber auch, dass die Geschlechterunterschiede in den Alternsverläufen sich über die Kohortenfolge der 1930er, der 1940er und der 1950er Geborenen hinweg nicht verringern.

Die Wahrscheinlichkeit, eine ehrenamtliche Tätigkeit neu aufzunehmen, nimmt bis zu einem Alter von 54 Jahren zu, und sie sinkt dann mit dem Älterwerden ab. Die Beendigung bleibt hingegen im Alternsverlauf stabil. Dadurch ergibt sich die stetig geringere Wahrscheinlichkeit, im höheren Alter einer ehrenamtlichen Tätigkeit nachzugehen. Die Prozesse der Aufnahme von Ehrenämtern unterscheiden sich nicht statistisch signifikant nach Geschlecht und Beendigungsprozesse unterscheiden sich nur minimal nach Geschlecht, mit einer geringfügig höheren Wahrscheinlichkeit bei Männern. Auch im Kohortenvergleich sind keine signifikanten Unterschiede zu erkennen. Die Wahrscheinlichkeit, ein Ehrenamt neu aufzunehmen oder zu beenden, ist für später geborene Kohorten über den gesamten betrachteten Alternsverlauf nicht höher beziehungsweise nicht niedriger als für früher geborene Kohorten.

\section{Interpretation}

Der Anstieg der Wahrscheinlichkeit, ein Ehrenamt auszuüben, zu Beginn der zweiten Lebenshälfte, kann für Frauen und für Männer unter anderem mit Gelegenheiten zum Ehrenamt, die sich durch die berufliche Tätigkeit ergeben, zusammenhängen. Menschen engagieren sich zum Beispiel in Berufsverbänden oder in berufsnahen Tätigkeitsfeldern ehrenamtlich oder der Arbeitgeber ermöglicht eine ehrenamtliche Tätigkeit: Einige Ärztinnen und Ärzte übernehmen etwa ehrenamtlich die Behandlung von Obdachlosen oder Schutzsuchenden, manche Pädagoginnen und Pädagogen kümmern sich um ehrenamtliche Bildungsangebote für Schülerinnen und Schüler mit besonderen Bedarfen. In manchen Betrieben besteht auch die Möglichkeit, sich für Auszubildende mit besonderen Bedarfen etwa durch die Organisation von Lerngruppen oder Trainingsgruppen ehrenamtlich einzusetzen. Der Rückgang der Ehrenamtswahrscheinlichkeit im höheren Alter kann für Frauen und für Männer etwa durch die Beendigung der beruflichen Tätigkeit und dem Wegfall der damit einhergehenden Gelegenheiten zum Ehrenamt, durch gesundheitliche Beeinträchtigungen (siehe Kapitel 3 Funktionale und subjektive Gesundheit in diesem Band) oder durch das Erreichen von - in Gesetzen oder Satzungen festgeschriebenen - Altersgrenzen für ein spezifisches Engagement ebenso erklärt werden, wie durch den Wunsch der Engagierten, keine weiteren Verpflichtungen mehr übernehmen zu müssen.

Wie können die Geschlechterunterschiede bei der Wahrscheinlichkeit, ein Ehrenamt auszuüben, über den Alternsverlauf erklärt werden? Aus der Literatur ist bekannt, dass die Möglichkeiten, sich ehrenamtlich zu engagieren, stark mit Bildung zusammenhängen. Die Geschlechterunterschiede bei der Wahrscheinlichkeit, über den Verlauf der zweiten Lebenshälfte ein Ehrenamt auszuüben, lassen sich zu einem großen Teil auf Bildungsunterschiede zwischen älteren Frauen und Männern zurückführen. ${ }^{1}$ Anders ausgedrückt könnte man sagen, hätten ältere Frauen zu einem ähnlichen Anteil hohe Bildungsabschlüsse wie ältere Männer, soll-

1 Wenn wir in zusätzlichen Analysen die Bildungsniveaus der Männer und Frauen berücksichtigen, sind die Differenzen bei den Ehrenamtswahrschein-

lichkeiten zwischen Frauen und Männern ab einem Alter von 67 Jahren nicht mehr signifikant. 
ten sie sich zu gleichen Anteilen ehrenamtlich engagieren und nicht zu niedrigeren Anteilen.

Wie kann das Weiterbestehen der Geschlechterunterschiede beim ehrenamtlichen Engagement im Alternsverlauf in den nachfolgenden Kohorten erklärt werden? Historisch waren die Bildungschancen für Männer deutlich besser als für Frauen, so dass heute der Anteil von älteren Männern mit hoher Bildung deutlich höher ist als der Anteil von älteren Frauen (Statistisches Bundesamt 2018). Die jüngeren Frauen verfügen heute über ähnliche Bildungsabschlüsse wie jüngere Männer (Statistisches Bundesamt 2018). Obwohl sich in den jüngeren Kohorten der Bildungsunterschied zwischen Frauen und Männern deutlich reduziert hat, zeigen sich jedoch auch bei den jüngeren Kohorten geringere Ehrenamtswahrscheinlichkeiten über den Alternsverlauf bei Frauen als bei Männern. Eine Verringerung der Geschlechterunterschiede ist für das Ehrenamt nicht zu beobachten. Im Kohortenvergleich sind die Entwicklungen für Frauen und Männern ähnlich, so dass jüngere Kohorten für beide Geschlechter eine höhere Partizipation zeigen als früher geborene Kohorten. Dass die Bildungsangleichung nicht zu einer Angleichung von Frauen und Männern beim Ehrenamt führt, liegt unter anderem daran, dass Frauen nach wie vor stärker in familiale Verpflichtungen eingebunden sind als Männer. Diese scheinen sich negativ auf die Übernahme von Ehrenämtern in Organisationen und Vereinen auszuwirken. Die weiterbestehenden Geschlechterunterschiede liegen vermutlich aber unter anderem auch an dem Angebot der zivilgesellschaftlichen Organisationen, das sich mehr an Männer als an Frauen richtet, zum Beispiel gibt es mehr Sportvereine für Männer als für Frauen.

Wie kann der Anstieg der Wahrscheinlichkeit, sich zu engagieren, bei nachfolgenden Kohorten erklärt werden? Dass sowohl Frauen als auch Männer der später geborenen Kohorten eine höhere ehrenamtliche Partizipation im Alternsverlauf zeigen als Frauen und Männer der älteren (also früher geborenen) Kohorten, könnte als Ergebnis der Verbesserung des Gesundheitszustands in der zwei- ten Lebenshälfte (siehe Kapitel 3 Funktionale und subjektive Gesundheit in diesem Band) zu sehen sein. Sowohl Frauen als auch Männer profitieren von einer besseren Gesundheit. Der Geschlechterunterschied in der funktionalen Gesundheit ist allerdings auch nicht kleiner geworden in den später geborenen Kohorten. Also haben Frauen, besonders im höheren Alter, eine schlechtere funktionale Gesundheit als Männer. Das wiederum könnte dazu beitragen, dass die Geschlechterunterschiede in der Wahrscheinlichkeit, sich ehrenamtlich zu engagieren, im höheren Alter stabil geblieben sind. Darüber hinaus können die besseren Engagementchancen später geborener Kohorten auch als Ergebnis der verstärkten Engagementförderung durch die Politik interpretiert werden, allerdings hat diese Engagementförderung bislang nicht $\mathrm{zu}$ mehr Gleichstellung oder einer Verringerung der Geschlechterunterschiede im Ehrenamt geführt.

\section{Politische Implikationen}

Wie soll Engagementförderung in der zweiten Lebenshälfte geschehen? Deutlich geworden ist aus der Analyse der geschlechtsspezifischen Alternsverläufe, dass die Wahrscheinlichkeit, sich in einem bestimmten Alter ehrenamtlich zu engagieren, sowohl getrieben wird durch die Neuaufnahmen ehrenamtlicher Tätigkeiten als auch durch deren Beendigung. Hieraus folgt, dass politische Maßnahmen, die auf eine Verbesserung der Chancen, sich bis ins hohe Alter ehrenamtlich zu engagieren, abzielen, an zwei ganz unterschiedlichen Stellschrauben ansetzen können: 1) An der Verbesserung der Chancen, eine ehrenamtliche Tätigkeit neu aufzunehmen - und zwar insbesondere in einem höheren Lebensalter - bei Personengruppen, die sich bislang eher selten engagieren wie ältere Frauen mit geringer Bildung. 2) An der Verbesserung der Chancen, eine ehrenamtliche Tätigkeit länger auszuüben - auch bis in ein hohes Lebensalter - und nicht zu beenden, für alle Menschen, die sich bereits engagieren und länger engagiert bleiben wollen. Optimal wäre eine am Le- 
bensverlauf orientierte Kombination aus beidem. Menschen, die sich bislang in ihrem Leben noch nicht ehrenamtlich engagiert haben, im höheren Alter dazu in die Lage zu versetzen, wenn sie dies möchten, lenkt den Blick auf die sozial ungleichen Chancen zur gesellschaftlichen Teilhabe durch Ehrenamt etwa von Frauen und Männern.

Wie können die Rahmenbedingungen dafür verbessert werden, dass zum einen mehr Menschen ein Ehrenamt aufnehmen können - wenn sie das wollen - und zum anderen, wie mehr Frauen und Männern dabei unterstützt werden können, ein einmal aufgenommenes Ehrenamt länger im Leben ausüben zu können, und dies möglicherweise auch bis ins hohe Alter hinein? Insbesondere die Gruppe der älteren Arbeitnehmerinnen und Arbeitnehmer, könnte ermutigt werden, durch die Aufnahme eines ehrenamtlichen Engagements auch außerberuflich noch einmal einen Neubeginn zu wagen, der sich vielleicht auch positiv auf die Gestaltung des Übergangs in den Ruhestand auswirken könnte - durch eine Kontinuität im Ehrenamt über den Renteneintritt hinaus. Allerdings ist die Lebensphase um die 60 eine, die insbesondere bei Frauen auch durch private Sorgearbeiten stark belastet ist (vgl. Kapitel 6 Unbezahlte Sorgetätigkeiten in diesem Band). Maßnahmen, die die Neuaufnahme ehrenamtlicher Aufgaben erleichtern sollen, müssen also auch die geschlechterdifferenzierten Belastungen durch berufliche Tätigkeiten wie zum Beispiel in der Familie mit im Blick haben. Hierbei steht weniger die verfügbare Zeit beziehungsweise die aufzuwendende Zeit im Fokus, denn auch Personen, die in Vollzeit erwerbstätig sind, finden zu hohen Anteilen noch Zeit, ein Ehrenamt - das in der Regel nur wenige Stunden pro Woche in Anspruch nimmt - auszuüben. Entscheidend sind vielmehr die Flexibilität der Arrangements einerseits, hier könnten zivilgesellschaftliche Organisationen sicherlich ansetzen und zum Beispiel ihre festen Termine für Vorstandssitzungen etc. flexibilisieren beziehungsweise familienfreundlicher gestalten, und die Belastung durch die Tätigkeiten in verschiedenen Bereichen andererseits. Eine Entlastung für pflegende Angehörige könnte also bei- spielsweise ebenfalls indirekt zu einer höheren Ehrenamtsbeteiligung beitragen. Und wie durch jede Arbeit, können auch im Ehrenamt zeitliche und zum Teil psychische Belastungen durch die jeweilige Tätigkeit entstehen, die vielleicht noch stärker in den Blick der Forschung geraten sollten, um Hindernisse, die dem Engagiertbleiben im Wege stehen, abzubauen.

Maßnahmen, die das längere Engagiertbleiben der Ehrenamtlichen fördern, könnten vor allem für Vereine und Organisationen von Interesse sein, die sich Sorgen machen, genügend Nachwuchskräfte für Leitungs- und Vorstandsfunktionen zu finden. Hier könnten ältere Engagierte mit ihrer Erfahrung abhelfen. Maßnahmen, die darauf abzielen, das Beendigungsalter anzuheben, können indirekt wirken. Zum Beispiel wirken sich Maßnahmen, die zur Aufrechterhaltung der Gesundheit im Alter beitragen, auch positiv auf das Ehrenamt aus. Menschen, denen es gesundheitlich gut geht, können sich auch eher für andere engagieren als Menschen mit gesundheitlichen Einschränkungen. Sie können aber auch direkt wirken, zum Beispiel indem rechtliche und satzungsgemäße Altersgrenzen daraufhin überprüft werden, ob sie verändert, flexibilisiert oder abgeschafft werden können. Eine Abschaffung der Altersgrenzen im Ehrenamt alleine würde jedoch die Geschlechterungleichheit in den Alternsverläufen zementieren, denn jene, die nie begonnen haben, ein Ehrenamt auszuüben (Frauen häufiger als Männer), würde eine solche Maßnahme nicht erreichen. Auch an anderer Stelle sind gruppenunspezifische Maßnahmen möglicherweise sogar kontraproduktiv. Wenn Engagementförderung nach dem Gießkannenprinzip erfolgt, dann werden sich die sozialen Ungleichheiten etwa zwischen den Bildungsgruppen aber eben auch zwischen den Geschlechtern erhalten und reproduzieren, im schlimmsten Falle werden sie sich sogar vergrößern.

Für eine geschlechterdifferenzierende und geschlechtergerechte Engagement- und Altenpolitik ist hierbei von Interesse, ob engagierte Frauen andere Unterstützungsbedarfe haben, um ihr Engagement möglichst lange bis ins hohe Alter auf- 
rechterhalten zu können als Männer und ob an Engagement interessierte Frauen andere Unterstützung benötigen, um ein Ehrenamt aufzunehmen als Männer. Zum Beispiel sollten sich, um das Engagement der Frauen zu erhöhen, beziehungsweise um die Geschlechterunterschiede zu reduzieren, zivilgesellschaftliche Organisationen gezielter an Frauen richten. In den meisten Bereichen haben Frauen geringere Engagementquoten als Männer (vgl. Vogel et al. 2017: 115) und Männer übernehmen auch häufiger Leitungsfunktionen in den Organisationen (vgl. Hagen \& Simonson 2017: 315). $\mathrm{Zu}$ fragen ist, ob attraktivere Funktionen für Frauen in Organisationen zusammen mit einer familienfreundlicheren Gestaltung das ehrenamtliche Engagement der Frauen im Lebensverlauf erhöhen würden. Die spezifische Förderung von Chancen im Ehrenamt von Frauen sollte deshalb nicht auf die Lebensphase Alter begrenzt sein, sondern schon in Jugend- und mittleren Erwachsenenalter gezielt erfolgen.

\section{Literatur}

Abraham, K. G., Helms, S., \& Presser, S. (2009). How social processes distort measurement: The impact of survey nonresponse on estimates of volunteer work in the United States. American Journal of Sociology, 114(4), 1129-1165.

Alisch, M., Ritter, M., Glaser, R., \& Rubin, Y. (2018). Engagement im Bürgerhilfeverein als Balanceakt zwischen sinnvoller Freizeitgestaltung, sozialer Teilhabe und Selbstprofessionalisierung. In: S. Scherger \& C. Vogel (Hrsg.) Arbeit im Alter. Zur Bedeutung bezahlter und unbezahlter Tätigkeiten in der Lebensphase Ruhestand (S. 245-267). Wiesbaden: Springer VS.

Hagen, C., \& Simonson, J. (2017). Inhaltliche Ausgestaltung und Leitungsfunktionen im freiwilligen Engagement. In: J. Simonson, C. Vogel \& C. Tesch-Römer (Hrsg.) Freiwilliges Engagement in Deutschland. Der Deutsche Freiwilligensurvey 2014 (S. 299-331). Wiesbaden: Springer VS.

Künemund, H. (2006). Tätigkeiten und Engagement im Ruhestand. In: C. Tesch-Römer, H. Engstler \& S. Wurm (Hrsg.) Altwerden in Deutschland (S. 289-327). Wiesbaden: VS Verlag für Sozialwissenschaften.

Künemund, H., \& Schupp, J. (2008). Konjunkturen des Ehrenamts - Diskurse und Empirie. In: M. Erlinghagen \& K. Hank (Hrsg.) Produktives Altern und informelle Arbeit in modernen Gesellschaften (S. 145163). Wiesbaden: VS Verlag für Sozialwissenschaften.

Künemund, H., \& Vogel, C. (2018). Altersgrenzen. Theoretische Überlegungen und empirische Befunde zur Beendigung von Erwerbsarbeit und Ehrenamt. In: S. Scherger \& C. Vogel (Hrsg.) Arbeit im Alter. Zur Bedeutung bezahlter und unbezahlter Tätigkeiten in der Lebensphase Ruhestand (S. 75-98). Wiesbaden: Springer VS.

Maurer, S. (2018). Der Einfluss der früheren Erwerbstätigkeit auf freiwilliges Engagement im Ruhestand. In: S. Scherger \& C. Vogel (Hrsg.) Arbeit im Alter. Zur Bedeutung bezahlter und unbezahlter Tätigkeiten in der Lebensphase Ruhestand (S. 195-215). Wiesbaden: Springer VS.

Müller, D., \& Tesch-Römer, C. (2017). Früheres Engagement und Engagementbereitschaft. In: J. Simonson, C. Vogel \& C. Tesch-Römer (Hrsg.) Freiwilliges Engagement in Deutschland. Der Deutsche Freiwilligensurvey 2014 (S. 153-178). Wiesbaden: Springer VS.

Naumann, D., \& Romeu Gordo, L. (2010). Gesellschaftliche Partizipation: Erwerbstätigkeit, Ehrenamt und Bildung. In: A. Motel-Klingebiel, S. Wurm \& C. Tesch-Römer (Hrsg.) Altern im Wandel. Befunde des Deutschen Alterssurveys (DEAS) (S. 118-141). Stuttgart: Kohlhammer. 
Şaka, B. (2018). Einfluss der Kohortenzugehörigkeit auf das ehrenamtliche Engagement im Alter ab 50 Jahren. In: S. Scherger \& C. Vogel (Hrsg.) Arbeit im Alter. Zur Bedeutung bezahlter und unbezahlter Tätigkeiten in der Lebensphase Ruhestand (S. 269-293). Wiesbaden: Springer VS.

Simonson, J., \& Hameister, N. (2017). Sozioökonomischer Status und freiwilliges Engagement. In: J. Simonson, C. Vogel \& C. Tesch-Römer (Hrsg.) Freiwilliges Engagement in Deutschland. Der Deutsche Freiwilligensurvey 2014 (S. 439-464). Wiesbaden: Springer VS.

Simonson, J., \& Vogel, C. (2018/im Erscheinen). Freiwilliges Engagement im Alter. In: K. R. Schroeter, C. Vogel \& H. Künemund (Hrsg.) Handbuch Soziologie des Alter(n)s. Wiesbaden: Springer VS.

Statistisches Bundesamt (2018). Bildungsstand der Bevölkerung - Ergebnisse des Mikrozensus 2017. Wiesbaden: Statistisches Bundesamt.

Vogel, C., Hagen, C., Simonson, J., \& Tesch-Römer, C. (2017). Freiwilliges Engagement und öffentliche gemeinschaftliche Aktivität. In: J. Simonson, C. Vogel \& C. Tesch-Römer (Hrsg.) Freiwilliges Engagement in Deutschland. Der Deutsche Freiwilligensurvey 2014 (S. 91-150). Wiesbaden: Springer VS.

Wetzel, M., \& Simonson, J. (2017). Engagiert bis ins hohe Alter? Organisationsgebundenes ehrenamtliches Engagement in der zweiten Lebenshälfte. In: K. Mahne, J. K. Wolff, J. Simonson \& C. Tesch-Römer (Hrsg.) Altern im Wandel. Zwei Jahrzehnte Deutscher Alterssurvey (DEAS) (S. 81-95). Wiesbaden: Springer VS.

Open Access Dieses Kapitel wird unter der Creative Commons Namensnennung 4.0 International Lizenz (http:// creativecommons.org/licenses/by/4.0/deed.de) veröffentlicht, welche die Nutzung, Vervielfältigung, Bearbeitung, Verbreitung und Wiedergabe in jeglichem Medium und Format erlaubt, sofern Sie den/die ursprünglichen Autor(en) und die Quelle ordnungsgemäß nennen, einen Link zur Creative Commons Lizenz beifügen und angeben, ob Änderungen vorgenommen wurden.

Die in diesem Kapitel enthaltenen Bilder und sonstiges Drittmaterial unterliegen ebenfalls der genannten Creative Commons Lizenz, sofern sich aus der Abbildungslegende nichts anderes ergibt. Sofern das betreffende Material nicht unter der genannten Creative Commons Lizenz steht und die betreffende Handlung nicht nach gesetzlichen Vorschriften erlaubt ist, ist für die oben aufgeführten Weiterverwendungen des Materials die Einwilligung des jeweiligen Rechteinhabers einzuholen.

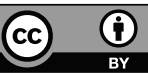




\title{
8. Frauen und Männer im Verlauf der zweiten Lebenshälfte: Eine Gesamt- betrachtung der empirischen Befunde
}

\author{
Frank Berner, Claudia Vogel, Markus Wettstein \\ und Clemens Tesch-Römer
}

In wissenschaftlichen und politischen Diskursen über das Älterwerden wird immer wieder argumentiert, dass Frauen und Männer auf sehr unterschiedliche Weise alt werden und dass das Älterwerden für Frauen etwas anderes bedeutet als für Männer. Im vorliegenden Bericht wurde anhand der längsschnittlichen Daten des Deutschen Alterssurveys (DEAS) untersucht, ob tatsächlich Unterschiede in den Alternsverläufen zwischen Frauen und Männern für verschiedene Lebensbereiche beobachtbar sind. Dazu wurden die Alternsverläufe von Frauen und Männern über eine Altersspanne vom 40. bis zum 90. Lebensjahr analysiert. Die folgenden Lebensbereiche mit insgesamt neun Merkmalen wurden in den vorangegangenen Kapiteln betrachtet: Gesundheit (funktionale und subjektive Gesundheit), psychisches Wohlbefinden (Lebenszufriedenheit und depressive Symptome), soziale Isolation und Einsamkeitserleben, Übernahme von Sorgetätigkeiten (die Betreuung von Enkelkindern und die Pflege und Unterstützung kranker Personen) sowie ehrenamtliches Engagement.

In diesem abschließenden Kapitel werden die Befunde aus den empirischen Kapiteln zusammengefasst, so dass eine Zusammenschau über das Äl- terwerden von Frauen und Männern in Deutschland resultiert. Alternsverläufe in den genannten fünf Lebensbereichen werden in diesem Bericht mit vier Fragestellungen untersucht:

1) Alternsverläufe: Wie verändern sich die betrachteten Lebensbereiche mit dem Älterwerden?

2) Geschlechterunterschiede in Alternsverläufen: Unterscheiden sich die Alternsverläufe der Frauen und der Männer, und wenn ja, in welcher Weise?

3) Alternsverläufe im sozialen Wandel: Verändern sich die Alternsverläufe im Wandel der Zeit? Unterscheiden sich also aufeinander folgende Geburtsjahrgänge in ihren Alternsverläufen?

4) Geschlechterunterschiede der Alternsverläufe im sozialen Wandel: Verändern sich die Unterschiede in den Alternsverläufen von Frauen und Männern im Wandel der Zeit (also über aufeinander folgende Geburtsjahrgänge)?

Im Folgenden werden die Ergebnisse, die im vorliegenden Bericht zu diesen vier Fragen erarbeitet wurden, zusammenfassend dargestellt und diskutiert. 


\subsection{Alternsverläufe}

Welche Veränderungen bringt das Älterwerden in verschiedenen Lebensbereichen mit sich? Die Ergebnisse der voranstehenden Kapitel geben Antworten auf diese Fragen. In Übersicht 1 sind diese zusammengestellt.

Man kann mehrere Gruppen von Alternsverläufen unterscheiden: Es gibt Merkmale, die sich mit dem Älterwerden zwischen dem 40. und dem 90. Lebensjahr nur in eine Richtung verändern: Die funktionale und die subjektive Gesundheit nehmen mit dem Älterwerden durchgehend ab; die soziale Isolation nimmt mit dem Älterwerden kontinuierlich $\mathrm{zu}$. $\mathrm{Zu}$ einer weiteren Gruppe gehören Merkmale, die mit dem Älterwerden zunächst zunehmen und später wieder abnehmen (glockenförmiger oder umgekehrt u-förmiger Verlauf). Dies ist der Fall bei der Lebenszufriedenheit, bei der Betreuung von Enkelkindern, bei der Pflege und Unterstützung kranker Personen und beim ehrenamtlichen Engagement. Eine weitere Gruppe enthält Merkmale, die mit dem Älterwerden zunächst abnehmen und später wieder zunehmen

\section{Übersicht 8-1 Alternsverläufe bezüglich verschiedener Merkmale}

\begin{tabular}{ll}
\hline Merkmal & Alternsverlauf \\
\hline Funktionale Gesundheit & $\begin{array}{l}\text { Die funktionale Gesundheit nimmt mit dem Älterwerden ab - und zwar beschleunigt: } \\
\text { Mit zunehmendem Alter nimmt die funktionale Gesundheit stärker ab. }\end{array}$ \\
Subjektive Gesundheit & Die Bewertung der eigenen Gesundheit nimmt mit zunehmendem Alter ab. \\
Lebenszufriedenheit & $\begin{array}{l}\text { Die Lebenszufriedenheit steigt vom 40. Lebensjahr bis zum Alter von etwa Mitte 70, } \\
\text { von da an sinkt die Lebenszufriedenheit wieder leicht. }\end{array}$ \\
Depressive Symptome & $\begin{array}{l}\text { Die Wahrscheinlichkeit für das Auftreten depressiver Symptome verläuft mit dem Älter- } \\
\text { werden u-förmig: Ab dem 40. Lebensjahr sinkt die Wahrscheinlichkeit für das Auftreten } \\
\text { depressiver Symptome, ab dem Alter von etwa Mitte 60 steigt sie wieder an. }\end{array}$
\end{tabular}

Soziale Isolation

Einsamkeit

Betreuung von Enkelkindern

Pflege und Unterstützung von kranken Personen

Ehrenamtliches Engagement
Die Wahrscheinlichkeit, sozial isoliert zu sein, steigt mit dem Älterwerden deutlich und fast kontinuierlich von etwa vier Prozent im Alter von 40 Jahren auf etwa 22 Prozent im Alter von 90 Jahren an.

Die Wahrscheinlichkeit, einsam zu sein, verläuft mit dem Älterwerden u-förmig: Im Alter von 40 Jahren liegt sie bei etwa elf Prozent, dann sinkt sie auf etwa acht Prozent im Alter von 70 Jahren, danach steigt sie wieder an bis auf etwa elf Prozent im Alter von 90 Jahren.

Die Wahrscheinlichkeit, Enkelkinder zu betreuen, verläuft mit dem Älterwerden glockenförmig (umgekehrt u-förmig): Im Alter von 40 Jahren liegt sie bei null Prozent, dann steigt sie bis zum Alter von etwa 65 Jahren auf etwa 24 Prozent und sinkt dann bis zum 90. Lebensjahr wieder auf fast null Prozent ab.

Die Wahrscheinlichkeit, kranke Personen zu unterstützen oder zu pflegen, steigt zwischen dem 40. und etwa dem 60. Lebensjahr von zehn Prozent auf etwa 16 Prozent an, um dann bis zum 90. Lebensjahr wieder bis auf etwa fünf Prozent abzusinken (umgekehrt u-förmiger Verlauf).

Die Wahrscheinlichkeit, sich ehrenamtlich zu engagieren, nimmt vom 40. Lebensjahr bis etwa zum 58. Lebensjahr von etwa 14 Prozent auf etwa 20 Prozent zu. Danach sinkt sie bis zum 90. Lebensjahr wieder bis auf etwa sechs Prozent ab (umgekehrt u-förmiger Verlauf). 
(u-förmiger Verlauf). Dies betrifft das Auftreten depressiver Symptome und das Auftreten von Einsamkeitsgefühlen.

Diese Ergebnisse helfen dabei, ein differenziertes Bild vom Älterwerden zu entwickeln. Zwar geht das Älterwerden schon ab dem mittleren Erwachsenenalter bis ins hohe Alter durchgängig mit einem Rückgang der funktionalen und der subjektiven Gesundheit sowie mit einer Zunahme der sozialen Isolation einher. Bis ins siebte Lebensjahrzehnt hinein entwickeln sich jedoch andere Merkmale auf eine Weise, die in der Regel positiv bewertet wird: In dieser Altersphase nimmt die Lebenszufriedenheit zu, die Wahrscheinlichkeit für das Auftreten von depressiven Symptomen und von Einsamkeitsgefühlen nimmt ab. Dazu kommt, dass in dieser Lebensspanne mit zunehmendem Alter auch zunehmend häufig Enkelkinder betreut werden und dass sich die Menschen mit zunehmendem Alter zunächst häufiger ehrenamtlich engagieren. Außerdem nimmt in dieser Phase die Unterstützung oder Pflege kranker Personen zu. Die drei zuletzt genannten Merkmale - die Betreuung von Enkelkindern, das ehrenamtliche Engagement und die Unterstützung/Pflege kranker Personen ist aus politischer Sicht häufig gewollt oder gewünscht, aus individueller Sicht kann jedoch gerade die Unterstützung oder Pflege kranker Angehöriger auch als eine Belastung empfunden werden.
Die meisten dieser Entwicklungen kehren sich im Verlauf oder ab dem Ende des siebten Lebensjahrzehnts (also im Alter zwischen dem 60. und dem 70. Lebensjahr) jedoch um. Dann sinkt die Lebenszufriedenheit leicht $a b$ und die Wahrscheinlichkeit für das Auftreten von depressiven Symptomen und von Einsamkeitsgefühlen steigt. Es sinkt auch die Wahrscheinlichkeit, Enkelkinder zu betreuen, sich ehrenamtlich zu engagieren oder sich um eine kranke Person zu kümmern. Bei diesen drei zuletzt genannten Entwicklungen sind verschiedene Einordnungen möglich: als Einbuße, weil man es nicht mehr schafft, oder auch als Entlastung und Entpflichtung.

Es wird dabei deutlich, dass das Älterwerden, zumindest wenn man es in der Spanne zwischen dem 40. und dem 90. Lebensjahr betrachtet, tatsächlich multidimensional und multidirektional ist und daher sehr unterschiedliche Facetten haben kann: Es gibt Anstiege und Rückgänge, kontinuierliche oder sich beschleunigende Entwicklungen, "Gewinne“ und „Verluste“. Je nach Merkmal sind die Alternsverläufe sehr unterschiedlich - was bei der Unterschiedlichkeit der Lebensbereiche auch keine große Überraschung ist. Dennoch zeigt das Gesamtbild, das sich hier ergibt, dass das Alter und das Älterwerden differenziert betrachtet werden müssen.

\subsection{Unterschiede zwischen Frauen und Männern}

Eine zentrale Frage des vorliegenden Berichts bezieht sich auf Unterschiede zwischen Frauen und Männern. Altern Frauen und Männer unterschiedlich? Verstärken sich die Unterschiede zwischen Frauen und Männern mit dem Älterwerden, werden sie im Verlauf des Älterwerdens kleiner, oder bleiben sie stabil? In Übersicht 2 sind die entsprechenden Befunde aus diesem Bericht zusammengefasst:

Die Übersicht 2 zeigt zunächst, dass bei fast allen der untersuchten Merkmale Unterschiede in den Alternsverläufen zwischen Frauen und Männern festgestellt wurden, die teilweise deutlich ausfielen. Legt man die hier untersuchten Merkmale zugrunde, altern Frauen also tatsächlich anders als Männer - ein Befund, der zwar nicht überrascht, aber hier doch eindrucksvoll über einen langen Untersuchungszeitraum und über verschiedene Lebensbereiche hinweg empirisch belegt wird. Lediglich bei der Einschätzung des eigenen Gesundheitszustandes und seiner Veränderung mit zunehmendem Alter unterscheiden sich Frauen und 
Übersicht 8-2 Unterschiede zwischen Frauen und Männern

\begin{tabular}{|c|c|}
\hline Merkmal & Geschlechterunterschied \\
\hline Funktionale Gesundheit & $\begin{array}{l}\text { Frauen haben über die gesamte zweite Lebenshälfte hinweg eine eingeschränktere funk- } \\
\text { tionale Gesundheit als Männer. Die funktionale Gesundheit nimmt bei Frauen zudem } \\
\text { deutlicher mit dem Älterwerden ab als bei Männern. Folglich nimmt der Unterschied } \\
\text { zwischen Frauen und Männern mit steigendem Alter zu. }\end{array}$ \\
\hline
\end{tabular}

Subjektive Gesundheit

Lebenszufriedenheit

Depressive Symptome

Soziale Isolation

Einsamkeit

Betreuung von Enkelkindern

Pflege und Unterstützung von kranken Personen

Ehrenamtliches Engagement
Kein signifikanter Geschlechterunterschied

Frauen sind zunächst zufriedener mit ihrem Leben als Männer. Mit dem Älterwerden nimmt ihre Zufriedenheit dann aber stärker ab als bei Männern. Im hohen Alter sind Frauen deshalb weniger zufrieden mit ihrem Leben als Männer.

Frauen haben ein höheres Depressionsrisiko als Männer. Bei beiden Geschlechtern nimmt die Wahrscheinlichkeit für das Auftreten depressiver Symptome mit dem Älterwerden zu, bei Frauen allerdings deutlich stärker als bei Männern.

Bei Frauen ist bis zu einem Alter von ca. 80 Jahren die Wahrscheinlichkeit, sozial isoliert zu sein, etwas geringer als bei Männern. Allerdings steigt bei Frauen diese Wahrscheinlichkeit mit dem Älterwerden stärker an als bei den Männern. Ab einem Alter von ca. 80 Jahren ist dann bei Frauen die Wahrscheinlichkeit, sozial isoliert zu sein, größer als bei Männern.

Bis etwa zur Mitte des siebten Lebensjahrzehnts ist bei Frauen die Wahrscheinlichkeit, einsam zu sein, geringer als bei Männern. Dieser Unterschied nimmt aber kontinuierlich ab. Ab einem Alter von 68 Jahren ist bei Frauen die Wahrscheinlichkeit, einsam zu sein, größer als bei Männern, dieser Unterschied nimmt mit dem Älterwerden noch zu.

Frauen betreuen Enkelkinder häufiger und früher in der zweiten Lebenshälfte als Männer. Dieser Unterschied wird mit dem Älterwerden kleiner, ab einem Alter von 70 Jahren gibt es keinen signifikanten Unterschied mehr zwischen Frauen und Männern.

Bei Frauen ist die Wahrscheinlichkeit, eine kranke Person zu unterstützen und zu pflegen, größer als bei Männern. Frauen unterstützen und pflegen auch früher in der zweiten Lebenshälfte als Männer. Im hohen Alter etwa ab 77 Jahren gibt es allerdings keine nennenswerten Unterschiede zwischen Frauen und Männern mehr.

Frauen haben über die gesamte zweite Lebenshälfte hinweg eine geringere Wahrscheinlichkeit als Männer, sich in Organisationen ehrenamtlich zu engagieren. Dieser Unterschied nimmt mit dem Älterwerden ab und ist im sehr hohen Alter ( $a b$ dem neunten Lebensjahrzehnt) nur noch sehr gering.
Männer nicht signifikant voneinander. Einige der beobachteten Unterschiede zwischen Frauen und Männern bleiben im Alternsverlauf stabil, einige Geschlechterunterschiede werden größer, einige Geschlechterunterschiede werden kleiner. Man kann unter anderem diese beiden Typen von Alternsveränderungen unterscheiden: (a) Stabile und sich vergrößernde Geschlechterunterschiede im Alternsverlauf

Es gibt Merkmale, bei denen die Unterschiede zwischen Frauen und Männern mit dem Älterwerden gleich groß bleiben oder bei denen sie sich sogar vergrößern. Dies betrifft die funktionale Gesundheit, das Auftreten depressiver Symptome, die soziale Isolation und Einsamkeitsgefühle. Die Merkmale beschreiben Zustände, Gefühle und 
Selbsteinschätzungen von Personen (Gesundheit, Depressivität, soziale Isolation und Einsamkeit).

\section{(b) Kleiner werdende Geschlechterunterschiede im Alternsverlauf \\ Es gibt Merkmale, bei denen die Unterschiede zwi- schen Frauen und Männern mit dem Älterwerden, insbesondere im Verlauf des sehr hohen Alters, kleiner werden. Dies betrifft die Betreuung von En- kelkindern, die Übernahme von Aufgaben bei der Pflege und Unterstützung kranker Personen und das ehrenamtliche Engagement. Hinsichtlich die- ser Merkmale scheint das sehr hohe Alter Frauen}

und Männer zu nivellieren. Diese Merkmale beschreiben Tätigkeiten, die Personen übernehmen oder übernommen haben (Enkelkinder betreuen, kranke Personen unterstützen oder pflegen, sich ehrenamtlich engagieren). Es gibt aber auch Lebensbereiche (etwa Betreuung von Enkelkindern und Unterstützung und Pflege kranker Personen), bei denen sich die Unterschiede zwischen Frauen und Männern bis zum mittleren Erwachsenenalter (etwa 40. bis 50. Lebensjahr) herausbilden und dann im Verlauf des sehr hohen Alters wieder kleiner werden.

\subsection{Unterschiede zwischen Geburtskohorten}

$\mathrm{Zu}$ jedem der genannten Merkmale wurde untersucht, welche Unterschiede in den Alternsverläufen es zwischen verschiedenen Geburtskohorten gibt. Dabei wurden die Geburtsjahrgänge zu drei 10-Jahres-Kohorten zusammengefasst: 1930-1939, 1940-1949 und 1950-1959.

Die Geburtsjahrgänge 1930 bis 1939 wurden während der Zeit des Nationalsozialismus sozialisiert und erlebten Kindheit und Jugendjahre zum großen Teil während des Zweiten Weltkriegs und unmittelbar danach. Im frühen Erwachsenenalter waren Angehörige dieser Geburtsjahrgänge die Träger des „Wirtschaftswunders“. Unterschiede in den Geschlechterrollen waren bei diesen Geburtsjahrgängen deutlich ausgeprägt.

Die Geburtsjahrgänge 1940 bis 1949 haben die Veränderungen der 68er Jahre als prägend erlebt - eine Zeit, in der auch die gesellschaftlichen Geschlechterverhältnisse eine Modernisierung erfahren haben. Die Erwerbs- und Familienbiografien dieser Geburtsjahrgänge wurden durch veränderte sozialpolitische Regelungen und den wirtschaftlichen Aufschwung im Nachkriegsdeutschland bestimmt.

Die Geburtsjahrgänge 1950 bis 1959 sind zu einem Teil bereits der Babyboomer-Generation zuzurechnen. Diese Geburtsjahrgänge profitierten von der Bildungsexpansion, so dass ein deutlich größerer Anteil von Frauen, aber auch von Männern höhere Bildungsabschlüsse erreichte. Darüber hinaus waren in den 1950er geborene Frauen seltener Hausfrauen und zu höheren Anteilen erwerbstätig als die früher geborenen Frauen.

Beim Vergleich der drei beschriebenen Kohorten wird zunächst nicht nach Frauen und Männern unterschieden. Die folgende Übersicht fasst also die Ergebnisse des Kohortenvergleichs der allgemeinen Alternsverläufe zusammen:

Tatsächlich unterscheiden sich die Alternsverläufe hinsichtlich der meisten der untersuchten Merkmale von Kohorte zu Kohorte. Lediglich beim Verlauf der subjektiven Gesundheit und der depressiven Symptome gibt es keine signifikanten Unterschiede zwischen den Kohorten. Der soziale Wandel hat einige Entwicklungen mit sich gebracht, die in der Regel positiv bewertet und zum Teil auch politisch gewollt werden: Nachfolgende Geburtsjahrgänge sind weniger sozial isoliert, sie sind aktiver im ehrenamtlichen Engagement und stärker an der Pflege und Unterstützung von kranken Personen beteiligt. Außerdem werden sie voraussichtlich mit einer höheren Lebenszufriedenheit sowie einer besseren funktionalen Gesundheit ins hohe und sehr hohe Alter kommen. 
Übersicht 8-3 Unterschiede zwischen den drei Kohorten

\begin{tabular}{|c|c|}
\hline Merkmal & Kohortenunterschiede \\
\hline Funktionale Gesundheit & $\begin{array}{l}\mathrm{Zu} \text { Beginn der zweiten Lebenshälfte haben früher geborene Kohorten eine bessere funk- } \\
\text { tionale Gesundheit als später geborene Kohorten; mit dem Älterwerden nimmt ihre } \\
\text { funktionale Gesundheit jedoch deutlicher ab als dies für die später geborenen Kohorten } \\
\text { erwartet wird. Im hohen Alter werden deshalb die später geborenen Kohorten voraus- } \\
\text { sichtlich eine bessere funktionale Gesundheit aufweisen als früher geborene Kohorten. }\end{array}$ \\
\hline
\end{tabular}

Subjektive Gesundheit

Lebenszufriedenheit

Depressive Symptome

Soziale Isolation

Einsamkeit

Betreuung von Enkelkindern

Pflege und Unterstützung von kranken Personen

Ehrenamtliches Engagement
Kein signifikanter Unterschied zwischen den Kohorten.

Die Lebenszufriedenheit ist in der zweiten Hälfte des siebten Lebensjahrzehnts bei allen drei 10-Jahres-Kohorten etwa gleich hoch. Bei den früher geborenen Kohorten nimmt die Lebenszufriedenheit ab einem Alter von etwa 70 Jahren allerdings deutlich stärker $\mathrm{ab}$ als dies für die später geborenen Kohorten erwartet wird.

Kein signifikanter Unterschied zwischen den Kohorten.

Je später eine der untersuchten Kohorten geboren ist, desto früher im Leben ist die maximale Wahrscheinlichkeit für soziale Isolation erreicht und desto niedriger ist die maximale Wahrscheinlichkeit für soziale Isolation. Bei den später geborenen Personen tritt soziale Isolation also früher im Leben auf als bei früher geborenen Personen, gleichzeitig ist diese Wahrscheinlichkeit aber geringer als bei den früher geborenen Personen.

Auch beim Verlauf des Einsamkeitsrisikos gibt es Unterschiede zwischen den drei 10-Jahres-Kohorten: Tendenziell verringert sich von Kohorte zu Kohorte die Wahrscheinlichkeit, einsam zu sein.

Je später eine der untersuchten Kohorten geboren ist, desto später in der zweiten Lebenshälfte werden Enkelkinder betreut.

Je später eine der untersuchten Kohorten geboren ist, desto größer ist die Wahrscheinlichkeit, kranke Personen zu unterstützen und zu pflegen. Und je später eine der untersuchten Kohorten geboren ist, desto früher im Alternsverlauf wird die Unterstützung und Pflege kranker Personen übernommen.

Je später eine der untersuchten Kohorten geboren ist, desto größer ist die Wahrscheinlichkeit, sich ehrenamtlich zu engagieren. Und je später eine Kohorte geboren ist, desto später in der zweiten Lebenshälfte sinkt die Wahrscheinlichkeit für ehrenamtliches Engagement.
Dass die Wahrscheinlichkeit für Pflege und Unterstützung kranker Personen bei später geborenen Personen früher im Leben ansteigt und auch insgesamt anwächst, entspricht dem politischen Paradigma der Betreuung von Pflegebedürftigen durch die Angehörigen. Diese Entwicklung geht aus individueller Sicht jedoch auch mit einer steigenden Belastung der Pflege- und Unterstützungspersonen einher. Im Wandel der Zeit gibt es also nicht nur Verbesserungen. So könnten von Kohorte zu Kohorte auch die Ansprüche an die eigene Ge- sundheit gestiegen sein, weshalb nachfolgende Geburtsjahrgänge keine bessere subjektive Gesundheit aufweisen als frühere Jahrgänge, obwohl für sie eine bessere funktionale Gesundheit im hohen Alter erwartet werden kann als bei früher geborenen Jahrgängen. Auch das Depressionsrisiko ist über nachfolgende Geburtsjahrgänge nicht geringer geworden, womöglich, weil der soziale Wandel keine Reduzierung der Risikofaktoren von Depression mit sich gebracht hat. 


\subsection{Unterschiede zwischen Frauen und Männern von Kohorte zu Kohorte}

Zudem wurden die geschlechtsspezifischen Unterschiede hinsichtlich der untersuchten Merkmale getrennt nach Kohorten untersucht. Damit konnte der Frage nachgegangen werden, ob und wie sich Unterschiede zwischen Frauen und Männern von Kohorte zu Kohorte verändern. Übersicht 4 zeigt die Ergebnisse dieser Analysen:

Im Abschnitt 8.2 wurde gezeigt, dass sich von den in diesem Bericht untersuchten Merkmalen lediglich bei der subjektiven Gesundheit kein Unterschied zwischen den Alternsverläufen von Frauen und von Männern zeigt. Auch bei getrennter Betrachtung der drei Kohorten zeigen sich für die subjektive Gesundheit keine Unterschiede zwischen den Alternsverläufen von Frauen und von Männern. Es kann überdies festgehalten werden, dass bei keinem der in diesem Bericht untersuchten Merkmale die Unterschiede zwischen den Alternsverläufen von Frauen und den Alternsverläufen von Männern von Kohorte zu Kohorte zunehmen. Insgesamt sticht der folgende Befund ins Auge: Bei fast allen Merkmalen, hinsichtlich derer sich Frauen und Männer in ihren jeweiligen Alternsverläufen unterscheiden, verändern sich diese Unterschiede von Kohorte zu Kohorte nicht oder nur unwesentlich. Bei keinem Merkmal nehmen die Geschlechterunterschiede in den Alternsverläufen also nennenswert ab oder zu.

Es hat sich gezeigt, dass sich die Alternsverläufe hinsichtlich der meisten Merkmale von Kohorte zu Kohorte zum Teil deutlich unterscheiden (siehe oben, Abschnitt 8.3). Der soziale Wandel zeigt sich also in der Veränderung der Alternsverläu$\mathrm{fe}$ in den verschiedenen Lebensbereichen. Doch bei fast allen untersuchten Merkmalen sind die Unterschiede zwischen den Alternsverläufen von Frauen und von Männern über alle drei Kohorten hinweg jedoch relativ stabil. Lediglich die Betreuung von Enkelkindern bildet hier eine Ausnahme, allerdings nähern sich die Geschlechter

Übersicht 8-4 Veränderung der Geschlechterunterschiede von Kohorte zu Kohorte

\begin{tabular}{lll}
\hline Merkmal & $\begin{array}{l}\text { Kleiner werdende Geschlechterunter- } \\
\text { schiede von Kohorte zu Kohorte }\end{array}$ & $\begin{array}{l}\text { Gleich bleibende Geschlechterunter- } \\
\text { schiede von Kohorte zu Kohorte }\end{array}$ \\
\hline Funktionale Gesundheit & $\begin{array}{l}\text { Kein Geschlechterunterschied, keine } \\
\text { Veränderung von Kohorte zu Kohorte }\end{array}$ \\
Subjektive Gesundheit & \\
Lebenszufriedenheit & $\checkmark$ \\
Depressive Symptome & $\checkmark$ \\
Soziale Isolation & $\checkmark$ \\
Einsamkeit & $\checkmark$ & $\checkmark$ \\
Betreuung von Enkelkindern & & $\checkmark$ \\
Pflege und Unterstützung von \\
kranken Personen \\
Ehrenamtliches Engagement
\end{tabular}


hier nicht in ihren Verläufen an, sondern der Geschlechterunterschied im Alter von 60 Jahren wird über nachfolgende Kohorten kleiner. Dies bedeutet, dass sich im sozialen Wandel, der sich in den Unterschieden der Alternsverläufe von Kohorte zu Kohorte ausdrückt, die Alternsverläufe von Frauen und von Männern auf gleiche oder zumindest sehr ähnliche Weise verändern. Der soziale Wandel geht nicht mit einer Angleichung der Alternsverläufe von Frauen und von Männern einher.

Dieses Fazit kann nur für die drei hier untersuchten, chronologisch nah beieinander liegenden Geburtsjahrgänge getroffen werden. Bei wei- ter auseinander liegenden Kohorten und einem noch längeren Untersuchungszeitraum könnten sich künftig womöglich deutlichere Veränderungen von Geschlechterunterschieden zeigen. Es liegt insbesondere die Vermutung nahe, dass sich für noch später geborene Kohorten und auch in anderen Lebensbereichen und Merkmalen eine Angleichung der Alternsverläufe von Frauen und Männern zeigt. Diese These kann jedoch erst in Zukunft untersucht werden, wenn eine entsprechende Datenbasis im Deutschen Alterssurvey vorliegt.

\subsection{Politische Implikationen}

\section{Die Wirkung gleichstellungspolitischer Maßnahmen}

Die deutlichen Unterschiede zwischen Frauen und Männern bei der Betreuung von Enkelkindern und bei der Pflege und Unterstützung von kranken Personen sind nicht überraschend. Die Ergebnisse vieler anderer Studien und die Alltagserfahrung vieler Menschen werden hier eindrucksvoll bestätigt. Auch hinsichtlich der Übernahme von Sorgetätigkeiten wurden in den letzten Jahrzehnten Anstrengungen unternommen, die Unterschiede zwischen Frauen und Männern auszugleichen. Ob dies gelingt, wird sich erst in zukünftigen Analysen mit Daten des Deutschen Alterssurveys zeigen. Zumindest bei der Enkelkinderbetreuung zeigt sich bereits auf Grundlage der verfügbaren Daten, dass sich Unterschiede zwischen Frauen und Männern tatsächlich verringert haben.

Der vorliegende Bericht wirft die Frage auf, ob und wie Gleichstellungspolitik auf das Leben im Verlauf der zweiten Lebenshälfte wirkt. In der Regel wirken gleichstellungspolitische Maßnahmen auf die Arbeitsteilung innerhalb von Familien, auf die Erwerbstätigkeit und das Einkommen von Frauen während des Erwerbslebens, damit indirekt auf die Einkommensverteilung zwischen
Frauen und Männern im Alter. Aber kann man erwarten, dass sich gleichstellungspolitische Maßnahmen darauf auswirken, wie sich Frauen und Männer innerhalb von Familien die Übernahme von Sorgetätigkeiten im höheren Lebensalter aufteilen? Die Betreuung und Pflege von Menschen mit eingeschränkter Gesundheit übernehmen immer noch vor allem Frauen. Hier wirken wahrscheinlich neben unterschiedlichen Verdienstmöglichkeiten von Frauen und Männern nach wie vor traditionelle Rollenbilder sowie die eingeübte Lebenspraxis. Gleichstellungspolitische Maßnahmen, die auf eine gerechtere Aufteilung von Sorgetätigkeiten zwischen Frauen und Männern abzielen, sollten deshalb an verschiedenen Stellen ansetzen. Unter anderem sollte auch Männern die Bedeutung dieser Tätigkeiten verdeutlicht werden. Möglicherweise halten sich manche Männer auch für nicht ausreichend kompetent, um Betreuungsund Pflegeaufgaben zu übernehmen. Angebote zur Weiterbildung könnten hier Wege sein, die Beteiligung von Männern in der informellen Pflege zu erhöhen. Insgesamt sollten Männer stärker dabei unterstützt werden, Sorgetätigkeiten zu übernehmen. 


\section{Fokus auf Risikogruppen}

Es ist bekannt, dass ältere Menschen - Frauen und Männer - ein hohes und im Verlauf des Älterwerdens steigendes Risiko haben, an gesundheitlichen Einbußen zu leiden. Allerdings ist das Gesundheitssystem als Ganzes längst nicht optimal auf die Versorgung älterer Menschen ausgelegt. Gesundheitspolitische Empfehlungen zu einer besseren Versorgung älterer Menschen wurden vielfach vorgelegt (siehe die entsprechenden Empfehlungen des Vierten, Sechsten und Siebten Altenberichts). Zwei Risikogruppen sollen im Folgenden hervorgehoben werden: Menschen mit ausgeprägter depressiver Symptomatik sowie chronisch einsame alte Menschen. Die Prävalenz dieser Risiken ist relativ klein (sie beträgt jeweils weniger als 20 Prozent). Aufgrund der stark negativen Auswirkungen von Depressionen oder Einsamkeitsgefühlen sollten diese Risikogruppen aber stärker in den Blick von Senioren- und Gesundheitspolitik genommen werden.

\section{(a) Depressive Symptomatik}

Das Risiko, an klinisch auffällig depressiven Symptomen zu leiden, liegt im sehr hohen Alter, wenn Menschen das 90. Lebensjahr erreichen, bei 16 Prozent für Frauen und bei sechs Prozent für Männer. Auch im sehr hohen Alter ist also die Wahrscheinlichkeit, von schweren depressiven Symptomen betroffen zu sein, eher gering. Andererseits können die Auswirkungen einer depressiven Erkrankung gravierend sein, so kann neben der allgemeinen Lebensqualität auch die körperliche Gesundheit beeinträchtigt werden und das Risiko kognitiver Einbußen kann ansteigen. Hinzu kommt, dass für die hier betrachteten Geburtskohorten bei nachfolgenden Geburtsjahrgängen kein Rückgang im Depressionsrisiko zu erkennen ist. Der soziale Wandel ging also offenbar nicht mit einer Verringerung des Depressionsrisikos in der zweiten Lebenshälfte und im sehr hohen Alter einher. Das gilt ebenso für die Unterschiede zwischen Frauen und Männern im Hinblick auf das Depressionsrisiko: Frauen aus späteren Geburtsjahrgängen werden voraussichtlich weiterhin mit einem deutlich höheren Depressionsrisiko ins hohe und sehr hohe Alter kommen als Männer. Frauen, die ein sehr hohes Alter erreichen, bilden also eine Risikogruppe für depressive Erkrankungen, die von mehr Unterstützungsangeboten zur Behandlung und Prävention dieser Erkrankungen profitieren könnte.

Dies sollte Anlass sein, stärker über eine angemessene gesundheitliche Versorgung dieser Gruppe nachzudenken. Allgemeinmediziner und -medizinerinnen sind Gatekeeper für das Erkennen von Warnsignalen und die Überweisung zu Fachärztinnen und -ärzten. Hier sollte es mehr Wissen darüber geben, wie klinisch relevante Depressivitätssymptome - gerade bei älteren Frauen - zu erkennen sind (etwa über Screening-Instrumente). Zugleich sollte es stärkere Anreize geben, diese diagnostischen Maßnahmen durchzuführen. Aufseiten der Fachmedizin (Psychosomatik und Psychiatrie) sowie der klinischen Psychologie (Psychotherapie) sollte die teilweise vorherrschende Denkweise des „klinischen Nihilismus“ überwunden werden, die davon ausgeht, dass Psychotherapien bei älteren Menschen nur noch wenig Wirkung zeitigen. Auch hier ist es notwendig, mit Hilfe von Anreizen die Versorgung von älteren Menschen mit stark ausgeprägten depressiven Symptomen zu verbessern.

\section{(b) Soziale Isolation und Einsamkeit}

Zwar zeigen die Analysen des Deutschen Alterssurveys, dass von einer Ausbreitung der Einsamkeit und der sozialen Isolation keine Rede sein kann: Eine deutliche Mehrheit aller Menschen ist auch im hohen Alter weder sozial isoliert noch einsam. Die Wahrscheinlichkeit, sozial isoliert und einsam zu sein, nimmt zudem über die Kohorten hinweg nicht zu. Man kann allerdings auch argumentieren, dass bei den 90-Jährigen eine Wahrscheinlichkeit von fast 20 Prozent für soziale Isolation und eine Wahrscheinlichkeit von über elf Prozent für Einsamkeit zu groß sind. Je größer der Anteil Hochbetagter an der Bevölkerung wird, umso mehr Personen wird dies betreffen.

Soziale Isolation ist nicht nur problematisch, 
weil man sich einsam fühlen kann, sondern auch, weil man niemanden oder nur wenige Menschen hat, an die man sich wenden kann, wenn man etwa aufgrund gesundheitlicher Einbußen Unterstützung braucht, um den Alltag zu bewältigen. $\mathrm{Zu}$ dem ist Einsamkeit ein erheblicher Risikofaktor für das Entstehen von Erkrankungen und vorzeitige Mortalität. Im hohen Alter gefährden daher soziale Isolation und Einsamkeit die Aufrechterhaltung eines selbstständigen Lebens.

Auch wenn man den Daten des Deutschen Alterssurveys zufolge sozial isoliert sein kann, ohne sich einsam zu fühlen, sollte es ein politisches Ziel sein, sozialer Isolation und Einsamkeit entgegen zu wirken. Soziale Isolation und Einsamkeit sind eine Herausforderung für Unterstützungssysteme in der Nachbarschaft, im Quartier und in der Kommune. Hier ist die Förderung zugehender Dienste notwendig, wobei zu beachten ist, dass sich im Verlauf chronischer Einsamkeit nicht selten Abwehrhaltungen und Misstrauen entwickeln. Die Kontaktaufnahme mit isolierten und einsamen Menschen ist deshalb unter Umständen nicht einfach zu bewerkstelligen. Zudem ist es notwendig, die Privatsphäre und Autonomie jener Personen zu achten, die dabei unterstützt werden sollen, aus dem Teufelskreis von Einsamkeit und Isolation herauszukommen. Dabei gibt es keine Patentrezepte für geeignete Interventionen: Nicht immer ist es ein Mangel an Kontaktmöglichkeiten, der zu Einsamkeit führt. Schließlich sollte unbedingt vermieden werden, Interventionen gegen Einsamkeit einseitig auf das hohe Alter auszurichten. Denn erstens können dadurch negative Altersbilder verstärkt werden, und zweitens ist Einsamkeit ein Problem aller Altersgruppen. Bei jüngeren Personen etwa könnten entsprechende Interventionen auch präventiv wirken und ihr Einsamkeitsrisiko senken, wenn diese das hohe Alter erreichen. Daher müssen Interventionen auch für alle Altersgruppen entwickelt werden.

\section{Förderung der gesellschaftlichen Partizipation}

Die Politik kann die gesellschaftliche Partizipation älterer Menschen unterstützen. Vor allem aber ist es das gestiegene Gesundheits- und Aktivitätspotenzial der „jungen Alten“ (etwa 60 bis 80 Jahre), das diese Aktivitäten möglich macht. In den vergangenen Jahren ist ein deutlicher Anstieg der Engagement- und Ehrenamtsquoten zu verzeichnen, auch bei Menschen in der zweiten Lebenshälfte. Offensichtlich haben engagementpolitische Maßnahmen gewirkt, und zwar bei Frauen und Männern gleichermaßen. Auch die beobachteten positiven Kohortentrends hinsichtlich der funktionalen Gesundheit im höheren Alter könnten eine Ursache dafür sein, dass das Engagement angestiegen ist.

Eine Angleichung von Frauen und Männern in den Alternsverläufen ehrenamtlicher Tätigkeiten ist nicht erkennbar. Würden bei engagementpolitischen Maßnahmen insbesondere Frauen in den Blick genommen, so könnte in diesem Bereich eine Angleichung zwischen den Geschlechtern erreicht werden. Wenn man davon ausgeht, dass freiwilliges und ehrenamtliches Engagement eine zentrale Form der gesellschaftlichen Partizipation im Alter ist, dann muss der Zugang dazu Frauen und Männern im gleichen Maße ermöglicht werden.

\section{Ausblick}

Im Vorwort zu diesem Buch haben wir eine wenig überraschende Aussage gemacht: Wenn wir älter werden, verändert sich unsere Lebenssituation in vielerlei Hinsicht. Allerdings wird diese Feststellung häufig ohne empirische Grundlage getroffen. Mit den Befunden im vorliegenden Buch legen wir eine solche empirische Grundlage vor. Gesundheit, psychisches Wohlbefinden, Einsamkeit und soziale Isolation sowie das Engagement in Sorgetätigkeiten und im Ehrenamt verändern sich über die zweite Lebenshälfte hinweg - zum Teil nur gering, zum Teil sehr deutlich. Zwischen Frauen und Männern zeigen sich viele Unterschiede, aber auch 
einige Ähnlichkeiten im Älterwerden, die im Verlauf der letzten Jahrzehnte relativ stabil geblieben sind. Es bleibt deshalb weiterhin auch eine Auf- gabe der Seniorenpolitik, die Gleichstellung zwischen Frauen und Männern in der Lebensphase Alter voranzutreiben.

Open Access Dieses Kapitel wird unter der Creative Commons Namensnennung 4.0 International Lizenz (http:// creativecommons.org/licenses/by/4.0/deed.de) veröffentlicht, welche die Nutzung, Vervielfältigung, Bearbeitung, Verbreitung und Wiedergabe in jeglichem Medium und Format erlaubt, sofern Sie den/die ursprünglichen Autor(en) und die Quelle ordnungsgemäß nennen, einen Link zur Creative Commons Lizenz beifügen und angeben, ob Änderungen vorgenommen wurden.

Die in diesem Kapitel enthaltenen Bilder und sonstiges Drittmaterial unterliegen ebenfalls der genannten Creative Commons Lizenz, sofern sich aus der Abbildungslegende nichts anderes ergibt. Sofern das betreffende Material nicht unter der genannten Creative Commons Lizenz steht und die betreffende Handlung nicht nach gesetzlichen Vorschriften erlaubt ist, ist für die oben aufgeführten Weiterverwendungen des Materials die Einwilligung des jeweiligen Rechteinhabers einzuholen.

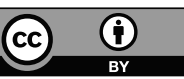




\section{Autorinnen und Autoren}

Dr. Frank Berner, Leiter des Arbeitsbereichs Politikberatung, Soziologe, Deutsches Zentrum für Altersfragen (DZA).

Anja Cengia, Wissenschaftliche Mitarbeiterin, Psychologin, Deutsches Zentrum für Altersfragen (DZA).

Heribert Engstler, Leiter des Arbeitsbereichs Wissenschaftliche Informationssysteme, Soziologe, Deutsches Zentrum für Altersfragen (DZA).

Dr. Oliver Huxhold, Leiter des Bereichs Methoden und Nachwuchsförderung, Psychologe, Deutsches Zentrum für Altersfragen (DZA).

Dr. Daniela Klaus, Wissenschaftliche Mitarbeiterin, Soziologin, Deutsches Zentrum für Altersfragen (DZA).

Dr. Laura Romeu Gordo, Stellvertretende Leiterin des Arbeitsbereichs Forschung, Ökonomin, Deutsches Zentrum für Altersfragen (DZA).

Dr. Svenja M. Spuling, Wissenschaftliche Mitarbeiterin, Psychologin, Deutsches Zentrum für Altersfragen (DZA).

Prof. Dr. Clemens Tesch-Römer, Institutsleiter, Psychologe, Deutsches Zentrum für Altersfragen (DZA).

Dr. Claudia Vogel, Leiterin des Deutschen Alterssurveys (DEAS), Soziologin, Deutsches Zentrum für Altersfragen (DZA).

Dr. Markus Wettstein, Stellvertretender Leiter des Deutschen Alterssurveys (DEAS), Psychologe, Deutsches Zentrum für Altersfragen (DZA). 